\title{
Respiratory mechanics in ventilated preterm infants: early determinants and outcome
}





\section{Respiratory mechanics in ventilated preterm infants: early determinants and outcome}

Mechanische eigenschappen van het respiratoire systeem bij beademde prematuren: vroege determinanten en gevolgen op langere termijn

(met een samenvatting in het Nederlands)

Proefschrift

ter verkrijging van de graad van doctor aan de Universiteit Utrecht

op gezag van de Rector Magnificus, Prof. Dr. W.H. Gispen, ingevolge het besluit van het College voor Promoties

in het openbaar te verdedigen op donderdag 27 november 2003 des middags te 12.45 uur

door

Dimphna Adriana Cornelia Maria Snepvangers geboren op 12 januari 1972, te Steenbergen 
$\begin{array}{ll}\text { Promotoren: } & \text { Prof. Dr. F. van Bel } \\ & \text { Prof. Dr. J.L.L. Kimpen }\end{array}$

Co-promotoren: Dr. C.K. van der Ent

Dr. J.P. de Winter

De voorkant van de omslag is een ontwerp van Annelies Snepvangers

Lay-out: Thea Schenk

Drukker: Labor Grafimedia B.V.

C2003. Niets van deze uitgave mag worden gecopieerd zonder toestemming van de auteur.

ISBN 90-901-7447-8 


\section{Contents}

CHAPTER 1

Perinatal lung development: normal course and effects

of various interrupting factors

CHAPTER 2

Respiratory function at neonatal age and during the first years of life

CHAPTER 3

Correction factors for oxygen and flow-rate effects on neonatal

Fleisch and Lilly pneumotachometers

CHAPTER 4

Chest radiograph scores in preterm infants: interobserver agreement and relation with respiratory function

CHAPTER 5

Perinatal lung development: normal course and effects of various interrupting factors

CHAPTER 6

Respiratory outcome in preterm ventilated infants: importance of early respiratory system resistance

CHAPTER 7

Neonatal respiratory mechanics and development of bronchial hyperresponsiveness in preterm infants

CHAPTER 8

Course of respiratory function in preterm ventilated infants during the first week and early years after birth

Summery \& Samenvatting

References

Curriculum vitae

Dankwoord 



\section{CHAPTER 1}

Perinatal lung development: normal course and effects of various interrupting factors 

During preterm birth, lung development is interrupted at a premature and vulnerable stage. Consequently, preterm infants often develop respiratory insufficiency for which they require ventilatory support at the neonatal intensive care unit (NICU). The premature transition from a safe intra-uterine environment to a relatively harmful environment in room air, containing higher levels of oxygen with or without ventilator support, is often complicated by acute (respiratory distress syndrome (RDS)) and chronic pulmonary diseases (chronic lung disease of prematurity (CLD)). Long-term respiratory morbidity and dysfunction depend on the degree of prematurity, perinatal complications, respiratory disease severity, and therapeutic interventions. In this chapter, normal human lung development will be discussed followed by a summary of respiratory complications related to prematurity.

\subsection{Normal human lung development}

\subsubsection{Antenatal lung development}

During human foetal lung development four stages can be identified: (1) the embryonic period, occurring between conception and the 5th week of gestation; (2) the pseudoglandular period, lasting from the 5th week to the 17th week; (3) the canalicular period, lasting from the 16th week to the 24th week; and (4) the terminal sac period, lasting from the 24th week until term. There is overlap from one stage to the next and the transition between stages occurs gradually (Figure 1) ${ }^{121}$.

From the laryngotracheal groove in the caudal end of the ventral wall of the primitive pharynx the lung bud becomes apparent at the end of the 4th week of gestation. ${ }^{121}$ As it grows caudally, two bronchial buds develop, which will be further branched into smaller bronchi. ${ }^{4}$ By 8 weeks gestation about 6 generations of branches exist consisting of 1 right and 1 left main bronchi, 5 lobar bronchi and 10 segmental bronchi. The thick epithelial wall of this tree of narrow tubules is composed of columnar or cuboidal epithelium. ${ }^{121}$

During the pseudoglandular period the primitive bronchi branch further until the bronchi terminalis (20th generation of branches) are developed in the 16th week of gestation. ${ }^{121,275}$ These pre-acinar airways may increase in size as further growth of the lung occurs, but new branches cannot be formed after the 16th week. ${ }^{121}$ Besides the development of the bronchial tree, the respiratory system starts developing with cuboid epithelial cells being (immature) type II alveolar cells at about 11 to 12 weeks of gestation. ${ }^{254}$ Bronchial smooth muscle, covering the branching epithelial tubules from early gestation, becomes innervated and spontaneously contractile from early on in gestation. Since the developing lung resembles an exocrine gland while intrauterine, elements involved with gas exchange are not formed yet. Consequently, foetuses born during the pseudoglandular period cannot survive. ${ }^{4}$ 


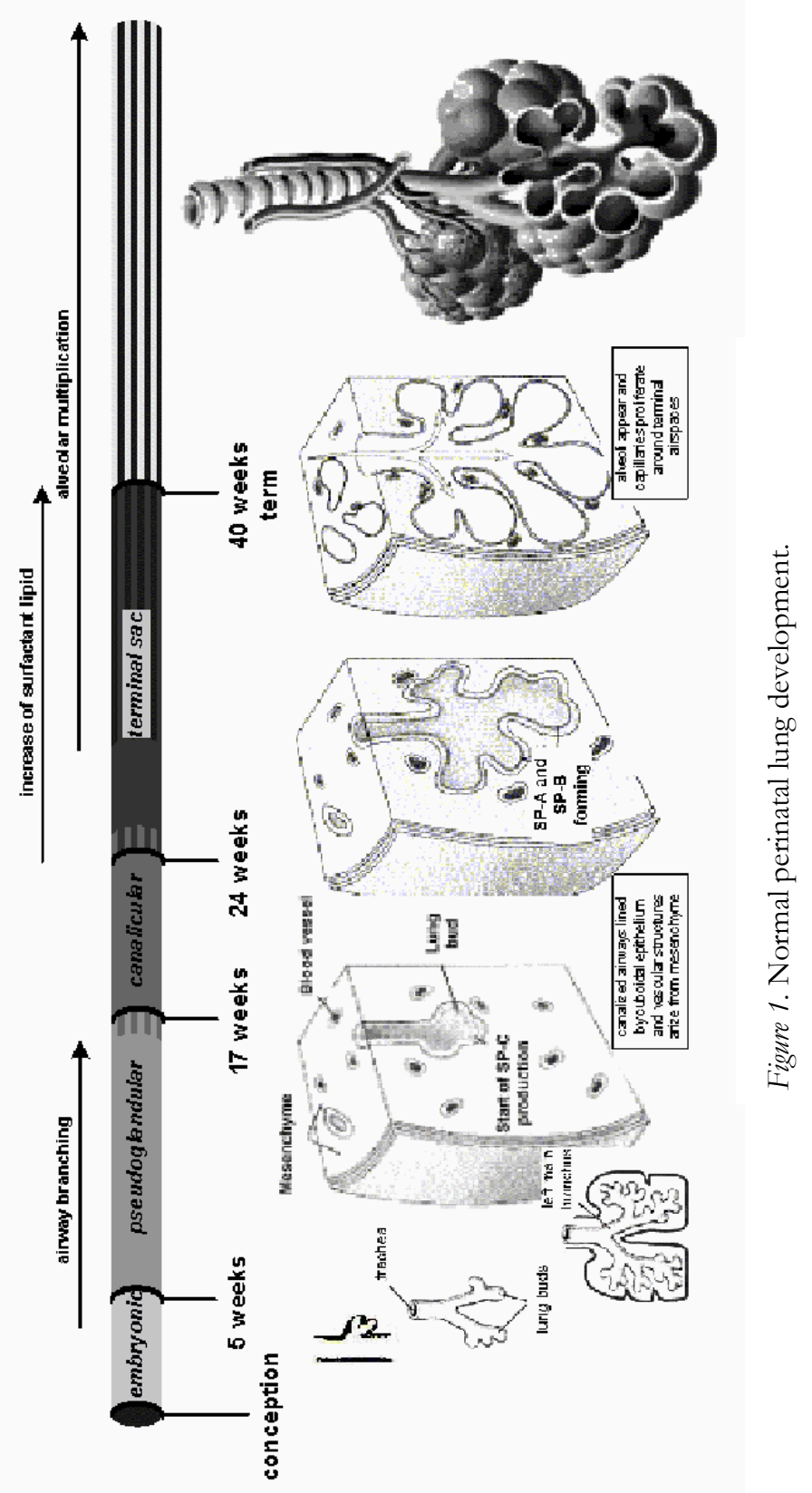


In the early canalicular stage from the 16th week of gestation, ${ }^{275}$ the early acini, i.e. the gas-exchanging portions of the tracheobronchial tree, become visible. Starting around 19 weeks of gestation, type II cells start to contain lamellar bodies, i.e. intracellular storage bodies of surfactant, and, partly, differentiate into flat type-1 epithelial cells.224 Although gas-exchange is possible, the saccules are not true alveoli, because they are larger, lack the smooth outline of the alveoli, and they are separated by thick septa containing large quantities of lipid and glycogen as well as a double capillary system surrounding a central core of loose connective tissue. ${ }^{121}$ Preterm infants born during this period predominantly depend upon these (sub)saccules to function as alveoli for pulmonary gas exchange. 275

During the fourth developmental period, the (sub)saccular or terminal sac period, from 24 weeks to term, further differentiation of the respiratory portion of the lung occurs. The saccules enlarge and form additional generations, of which the last generation is called the terminal saccule. From as early as the 29th week, the first alveoli may be recognized, ${ }^{115}$ but from 36 weeks gestation alveoli are uniformly developed with thin septa containing little glycogen and, eventually, one capillary plexus. ${ }^{108,121}$ The number of alveoli present at term varies between $10-149$ million. ${ }^{144}$

For normal antenatal lung development, the lungs need space, amniotic and foetal lung liquid, intraluminal fluid secretion and the stimulus of foetal breathing movements. ${ }^{144,224}$ The foetal lung, like the postnatal lung, has a tendency to recoil and, in order to grow, must be actively maintained in an expanded state. ${ }^{109}$ Overdistension of the foetal lungs with luminal liquid leads to an acceleration of tissue growth and an increased rate of thinning, or structural maturation, of the alveolar wall. ${ }^{177}$ Pulmonary surfactant is a surface-active material formed by alveolar type II cells lining the alveolar surface area of the lung and is stored in intracellular lamellar bodies. It is a lipid-protein complex, which consists of about 90 per cent lipids and 10 per cent surfactant-specific proteins. Surfactant proteins (SP)-B and SP-C are detectable early in gestation, before recognizable mature type- 2 cells or surfactant phospholipid can be detected (i.e. before 19 weeks of gestation). ${ }^{224}$ Although the function of all separate elements of surfactant is not yet completely elucidated, SP-B and SP-C seem to be involved in the regulation of surfactant phospholipid metabolism, accelerating the adsorption of phospholipid to the air-liquid surface lining the alveolar surface to reduce surface tension, ${ }^{108}$ whereas SP-A and SP-D are believed to have an important role in the defence of the lung against bacteria and viruses and parallel the development of the phospholipids from 20 to 24 weeks of gestation during which the type-2 cells with lamellar bodies appear. ${ }^{108}$ The phospholipid content of surfactant consists for a major part $(\approx 76$ per cent) of lecithin (saturated phosphatidylethanolamine).281 To assess degree of prenatal lung maturation determination of the lecithin/sphingomyelin ratio (L/S ratio) in amniotic fluid has become common practice. To correct for changes in amniotic fluid volume the concentration of lecithin is standardized to the concentration of sphingomyelin, which remains relatively 
constant throughout gestation. In normal pregnancy, the $\mathrm{L} / \mathrm{S}$ ratio displays a remarkably stable pattern, increasing slowly to 1.0 at 32 weeks, rising more rapidly to 2.0 at 35 weeks, and accelerating rapidly thereafter to reach a mean value of 5.0 at term. ${ }^{107}$ Saturated phosphatidyletanolamine content in the lung increases after about 24 weeks. During the latter part of gestation, there is an increase in lung adrenergic receptors, increasing the response to catecholamines followed by a stimulation of surfactant secretion. The density of adrenergic receptors is increased by corticosteroids, thyroid hormone, and oestrogen. ${ }^{108}$

\subsubsection{The transition during birth}

Before birth, the placenta is the organ of respiration and pulmonary vascular resistance is maintained at a very high level by the muscular pre-capillary vessels to shunt away all the venous return to the right heart through the oval foramen and the arterial duct. At birth, the umbilical cord is tied and cut, and the lungs must take over all respiratory function from the placenta within seconds or the baby will perish. The lung must convert from a secretory to an absorptive organ. The first step consists of inhibition of secretion of lung liquid by increased concentrations of catecholamines and other hormones. Subsequently, reabsorption occurs passively, secondary to differences in oncotic pressure between air spaces, the interstitium, and blood vessels, and actively, secondary to active transport of sodium out of the air space. Infants born prematurely or those born without labour do not have the opportunity for early lung liquid clearance, and they begin their extra-uterine life with excess water in the lungs. After birth, water in the air spaces moves rapidly to the extra-alveolar interstitium, where it pools in perivascular cuffs of tissue and in the interlobar fissures. It is then cleared gradually from the lung by lymphatic drainage or by absorption directly into the small blood vessels. ${ }^{107}$ Increase of circulating catecholamines also stimulates surfactant secretion, decreasing surface tension of the alveolar air-liquid surface and facilitating the recruitment of pulmonary air spaces after birth. The first breath results in vasodilatation by the mechanical effect of traction on the vasculature as the chest wall expands, by oxygen entering the lungs for the first time, cyclo-oxygenase metabolites and endothelin. Also, functional closure of the foetal shunts, i.e. oval foramen and arterial duct, occurs quickly, followed by a permanent anatomical closure. If the baby becomes even minimally hypoxic or acidaemic the pulmonary vascular resistance may rise steeply, causing reopening of the foetal shunts and rightto-left shunting with profound hypoxemia, a situation called 'persistent pulmonary hypertension of the neonate'. 224 


\subsubsection{Postnatal lung development}

To describe postnatal lung development from birth to adulthood, it may be useful to draw an analogy with the mathematical model of height development of Karlberg and colleagues. ${ }^{133,224}$ A three-phase model of height development in postnatal life was described: (1) a nutrition-dependent phase comprises a very rapid, but rapidly decelerating, growth lasting until the end of the second year equating the phase of alveolar multiplication; (2) a growth hormone-dependent childhood phase with almost linear growth until approximately 10 years of age without further multiplication of alveoli or change in airway numbers, but merely enlargement and elongation of already existing structures, producing linear changes in lung function with height; (3) a pubertal phase with a rapid change in height, which will slowly plateau off by approximately 20 years of age and is related to a three-dimensional enlargement of the lung without multiplication. ${ }^{224}$

Early postnatal lung growth is characterized by further formation of alveoli, maturation of lung structures, and production and secretion of a variety of substances within the lung. The age at the end of alveolarization is controversial. The current consensus is that alveolar formation starts at 26 weeks, that by term only 15 per cent of the adult component are present and that the bulk of the process of forming new alveoli is completed by 6 months and virtually complete by 2 to 3 years of age. 224,275 There are indications that, after this age, alveolar multiplication is limited or even absent and that alveoli only increase in size. ${ }^{215,257}$

Airways elongate and enlarge, while the relative amount of bronchial smooth muscle gradually increases at all levels until 8 months of age. ${ }^{115}$ After this age, the muscular coat of the peripheral bronchioles thins and is lost altogether in the terminal and respiratory bronchioles. This is thought to occur in a manner similar to fingers extending through a mitten glove, namely that the muscle coat stays where it is as the airways grow through them. ${ }^{224}$ Due to progressive alveolarization, early postnatal volume increase of air spaces is relatively larger then that of the airways, which is called dysanaptic or nonisotropic growth. ${ }^{121}$ As laminar airflow is inversely proportional to the fourth power of the radius and turbulent airflow is inversely proportional to the seventh power of the radius, very small changes in airway size have profound effects on airflow. 224

Although methodological differences may exist between older children and young infants concerning assessment of bronchial responsiveness, ${ }^{146,147}$ it is suggested that bronchial responsiveness decreases with age. Contractile forces of the airway smooth muscle, geometry of the airways, and impedance against which the airway smooth muscle must contract (cartilage and elastic recoil of the lung) change with age and affect airway reactivity. ${ }^{179}$ Additionally, forces of interdependence between pulmonary airways and parenchyma increase with age, limiting airway narrowing, ${ }^{252}$ 
and the number of cholinergic muscarinic receptors and their affinity for muscarinic agonists decrease with age while airway acetylcholinesterase activity increases. ${ }^{176}$

\subsection{Factors affecting lung development in preterm infants}

As was described in paragraph 1.1., growth and development of the lung follow a specific pattern. Consequently, environmental changes prior to the 16th week of intrauterine life can affect the number of conducting airways, while changes occurring after the 16th week will affect the size rather than the number of airways. Numbers of alveoli are modified if environmental changes occur during the period between the prenatal canalicular stage and postnatal completion of alveolar multiplication. ${ }^{221}$ Hence, pulmonary sequelae of preterm birth and their severity depend on the developmental stage at which preterm birth and ante- and postnatal interventions occur, but will never affect airway number, although it may affect subsequent airway growth. ${ }^{127}$ Respiratory problems following preterm birth mainly bear on the interruption of parenchymal development during a critical stage of alveolarization and vascularization. ${ }^{56,127}$ As a consequence, fewer and larger alveoli will develop. Additionally, capillary configuration will be abnormal and an adaptive dysmorphic pattern of vascular organization often exists. Since both the number of alveoli and capillaries are reduced, the alveolar-to-arterial ratio may be normal, ${ }^{257}$ but the significant and permanent loss of alveoli may not be catched up later on. So, the earlier lung development is interrupted, the higher the degree of alveolar hypoplasia will be. ${ }^{56}$ Furthermore, surfactant proteins are decreased relative to the lipids, surfactant function is decreased, and it is more sensitive to inhibition by serum proteins. Surfactant metabolism in the preterm newborn is characterized by slow secretion, minimal catabolism, and an efficient reuse of phospholipid and protein components. ${ }^{129}$

\subsubsection{Factors affecting antenatal lung development}

In the foetus, lung development can be affected by a range of physical, metabolic, endocrine and inflammatory factors affecting the intra-uterine environment (Figure 2).109,110 The impact of various congenital malformations on antenatal lung development depends on the time of occurrence. A congenital diaphragmatic hernia may develop during conducting airway formation very early during gestation and may therefore cause a reduction of the number of bronchial divisions. Since a hernia remains present during the latter stages of lung growth, airway size will also be diminished. Other examples include: congenital cystic lung disease or congenital bronchopulmonary-foregut malformations, including pulmonary sequestration, 


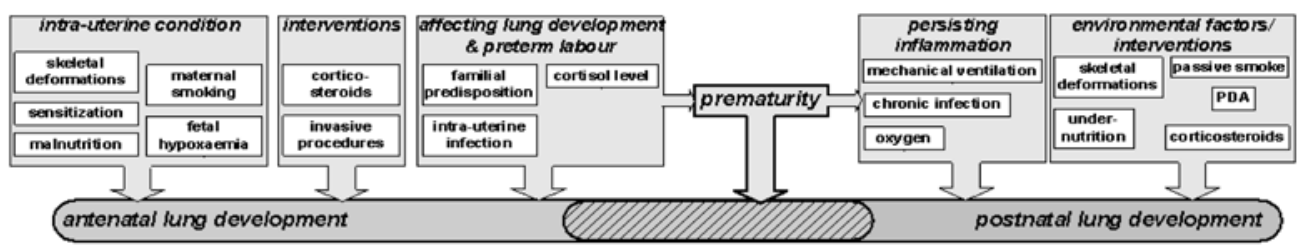

Figure 2. Factors affecting ante- and postnatal lung development.

congenital cystic adenomatoid malformation, and congenital bronchogenic cysts, and deformations of the thorax wall, like osteogenesis imperfecta. ${ }^{121}$

Factors affecting both lung development and foetal growth or preterm birth include foetal nutrient restriction, foetal hypoxemia, elevated circulating cortisol levels and intra-uterine infection. Because lung liquid secretion is an active process, it is possible that foetal hypoxemia or hypoglycaemia reduce amniotic fluid volume via reduced foetal production of urine and lung liquid. Additionally, foetal breathing movements are inhibited by acute hypoxemia and hypoglycaemia, although they may be normal in chronical situations of deficiency. Hypoxemia may inhibit growth and DNA synthesis, ${ }^{109}$ and may increase circulating concentrations of cortisol, ${ }^{23}$ which may affect the duration of gestation and organ development in the foetus, ${ }^{109}$ but may also stimulate lung maturation by increasing surfactant levels. ${ }^{110}$

Severe undernutrition can cause degenerative changes in the lung ${ }^{110,228}$ and an association has been described between maternal malnutrition during mid and early gestation and developing obstructive airway disease in adulthood. ${ }^{156}$ Besides dietary lipids that are important for normal development of surfactant synthesis and antioxidant systems, vitamin A and selenium are considered to be important for normal lung development. ${ }^{109}$ According to studies in small-for-gestational-age infants, ${ }_{109,110}$ effects of undernutrition on the developing lung include: reduction in lung weight, DNA or protein content, reduced surfactant content of lungs and its surface activity, impaired maturation of type- 2 alveolar cells, decreased alveolar formation, reduced surface area for gas exchange, immature, thicker air-blood barrier and alveolar wall, reduced elastin content, and reduced acid phosphatase in alveolar macrophages. Besides dietary lipids that are important for normal development of surfactant synthesis and anti-oxidant systems, vitamin $\mathrm{A}$ and selenium are considered to be important for normal lung development. ${ }^{109}$

Familial predisposition to asthma also seems to affect foetal airway development. ${ }^{243}$ There is increasing interest in the possibility of antenatal sensitization of the foetus, not merely to ingested allergens but even possibly to aeroallergens. ${ }^{224,276}$ 
Maternal smoking during pregnancy has been found to be adversely related to postnatal airway function, suggesting that cigarette smoke limits foetal airway growth. $118,155,243$ Surgical procedures including amniocentesis may also adversely affect foetal airway development. 88

Preterm labour is associated with intra-uterine infection in 45 percent of preterm births before 37th week of gestation. ${ }^{104}$ Increased degrees of prematurity are associated with an even higher incidence of intra-uterine infection. Bacteria in the genital tract and foetal membranes produce interleukines, IL-1 and TNF- $\alpha$, releasing chemokines, colony stimulating factors and prostaglandines that induce labour. ${ }^{129}$ The presence of intra-uterine infection increases the risk of foetal pulmonary inflammation. Postnatally, increased levels of pro-inflammatory cytokines IL-1, IL-6, IL- 8 and TNF- $\alpha$ have been found in lavage fluid of preterm infants. Neutrophil counts are increased and chemotactic activity is initiated. As a consequence of this pulmonary inflammation, increased capillary permeability causes protein leakage into alveoli that inhibit the local activity of surfactant. On the other hand, there is increasing evidence that intra-uterine pulmonary inflammation may result in increased pulmonary maturation, ${ }^{104}$ decreased RDS severity and improved lung mechanics. Increased IL- $1 \alpha$ is found to be associated with increased pulmonary compliance, and increased amounts of phospholipids and SP-B. ${ }^{159}$ At the same time, later progression to CLD may result from progression of the antenatal pro-inflammatory exposure. $126,222,277$

Multiple hormones and growth factors can modulate lung maturation in vitro systems. However, consistent and large effects have only been reported for in vivo evaluations of antenatal corticosteroid therapy and not for thyroid hormone or growth hormone. $38,129,147$ Antenatal corticosteroids are frequently and appropriately administered to mothers in preterm labour to mature the foetal lung and to decrease the risk of developing RDS. Glucocorticosteroids function primarily as modulators of development, ${ }^{129}$ promote differentiation of alveolar type II cells and increase the number of surfactant-containing lamellar bodies in these cells. Steroids up-regulate surfactant proteins A, B and C at the transcriptional level. ${ }^{129,224}$ However, this effect is transient, because mRNA levels return to baseline values within several days. ${ }^{10}$ Improved postnatal lung mechanics result from long-term effects, like thinning of the alveolar wall and increased potential lung gas volume, rather than from an increase of surfactant quantities or function. ${ }^{134}$ Furthermore, antenatal glucocorticoid administration also stimulates the direct transcription of epithelial sodium channels, improving fluid absorption at birth. One of the unwanted effects is that corticosteriods inhibit the growth of new interalveolar septa, probably as a consequence of reduced DNA and protein synthesis, ${ }^{109}$ resulting in an emphysematous state of the lung with a reduced number of enlarged alveoli.261 


\subsubsection{Factors affecting postnatal lung development: respiratory distress syndrome (RDS)}

As a consequence of respiratory problems and diseases following prematurity, intensive therapeutic measures are required which herald risks for postnatal lung development and will be discussed in the following sections.

\subsubsection{History and epidemiology of $\mathrm{RDS}$}

The first description of the clinical features and treatment of infants suffering from RDS was published as early as 1835 by Eduard Jörg and colleagues. ${ }^{132}$ But it took until 1903 to discover the presence of hyaline membranes in the lungs, considered to represent aspirated amniotic fluid. ${ }^{117}$ This view changed in the early 1950s, when it was suggested that hyaline membranes resulted from tissue damage and transudation as a secondary reaction to prematurity and foetal hypoxia..22,169 After 1954, evidence is increasing that major pulmonary function abnormalities coexist with hyaline membrane disease. ${ }^{33}$ Pulmonary surfactant deficiency was demonstrated for the first time in 1959 in infants who died from hyaline membrane disease. ${ }^{8}$ In the same year, the name of the syndrome was changed from 'idiopathic respiratory distress syndrome' into '(infant) respiratory distress syndrome', which has been used synonymously with hyaline membrane disease ever since. ${ }^{33}$

Before the ventilator era, the clinical course of RDS was remarkable in that death usually occurred within the first few days after birth. As the specialty of neonatology emerged in the 1950s and 1960s, more attention was given to supportive care of the infants, particularly with more accurate ventilators and the survival of these infants increased. In the late 1960s and early 1970s a further improvement of survival occurred mainly because micro determinations of blood gases became available and ventilators further improved. ${ }^{33}$ The introduction of continuous positive airway pressure (CPAP) resulted in markedly reduced RDS severity and mortality from the early 1970s. In 1973, it was demonstrated that RDS could be prevented by antenatal corticosteroid administration and in 1980, Fujiwara and colleagues ${ }^{74}$ demonstrated the effectiveness of artificial surfactant replacement therapy and this was followed by many authors publishing prospective, controlled, clinical trials promoting surfactant replacement therapy. ${ }^{73,119}$ Surfactant being administered at birth appeared to prevent RDS and in 1993 the synergistic effects of the combined use of prenatal corticosteroids and surfactant replacement were demonstrated. ${ }^{33}$

As a consequence of these improvements in perinatal care of preterm infants, severity of RDS gradually decreased during the past decades. ${ }^{56}$ This did not seem to affect the incidence rate in The Netherlands during past decades. (Table 1) However, the number of preterm births as well as the survival of very preterm infants increased at the same time. ${ }^{280}$ Since most deaths occur within the first days of life, ${ }^{280}$ RDS might not have been registered in these infants. Both incidence rate and RDS severity are still inversely proportional to gestational age and birth weight (Table 1). 
Table 1. Incidence rates of RDS and CLD in preterm infants ( $<37$ weeks gestation) according to time period, gestational age and birth weight.

\begin{tabular}{|c|c|c|c|c|c|}
\hline & $<28$ weeks & $<30$ weeks & $<32$ weeks & $\geq 32$ weeks & All \\
\hline \multicolumn{6}{|l|}{ RDS } \\
\hline internationally 1996136 & $60-80 \%$ & & & $15-30 \%$ & \\
\hline nationally $1983^{272}$ & & & $57 \%$ & & \\
\hline nationally $1993^{54}$ & $54 \%$ & $45 \%$ & $43 \%$ & $14 \%$ & $30 \%$ \\
\hline nationally $2001^{54}$ & $81 \%$ & $73 \%$ & $59 \%$ & $19 \%$ & $39 \%$ \\
\hline \multicolumn{6}{|l|}{ CLD } \\
\hline 1987163 & & & $25 \% / 11 \%$ & & \\
\hline $1992^{163}$ & & & $41 \% / 26 \%$ & & \\
\hline 1997163 & & & $42 \% / 29 \%$ & & \\
\hline 1989-1999163 & & & $40-45 \%$ & & \\
\hline nationally $1993^{54}$ & $30 \%$ & $20 \%$ & $14 \%$ & $0 \%$ & $7 \%$ \\
\hline \multirow[t]{2}{*}{ nationally $200^{154}$} & $24 \% / 8 \%$ & $17 \% / 6 \%$ & $10 \% / 4 \%$ & $0 \%$ & $2 \%$ \\
\hline & $<1000 \mathrm{~g}$ & \multicolumn{2}{|c|}{$1000-1500 \mathrm{~g}$} & $>1500 \mathrm{~g}$ & All \\
\hline \multicolumn{6}{|l|}{ RDS } \\
\hline nationally $1993^{54}$ & $49 \%$ & \multicolumn{2}{|c|}{$35 \%$} & $21 \%$ & $30 \%$ \\
\hline nationally $2001^{54}$ & $68 \%$ & \multicolumn{2}{|c|}{$52 \%$} & $24 \%$ & $39 \%$ \\
\hline \multicolumn{6}{|l|}{ CLD } \\
\hline $1967 a$ & $100 \%$ & \multicolumn{2}{|c|}{$43 \%$} & $27 \%$ & $28 \%$ \\
\hline 1989b,195 & $71 \%$ & \multirow{2}{*}{\multicolumn{2}{|c|}{$14 \%$}} & & $31 \%$ \\
\hline 1989-1991b,222 & $37 \%$ & & & & \\
\hline nationally $1993^{54}$ & $25 \%$ & \multicolumn{2}{|c|}{$8 \%$} & $1 \%$ & $7 \%$ \\
\hline nationally $2001^{54}$ & $19 \% / 9 \%$ & \multicolumn{2}{|c|}{$7 \% / 2 \%$} & $0 \%$ & $2 \%$ \\
\hline
\end{tabular}

a 'Classic' BPD, as described by Northway and colleagues. ${ }^{19}$

b CLD defined as supplemental oxygen requirement for 28 days after birth.

c Including infants meeting one of both definitions of CLD: supplemental oxygen requirement for 28 days after birth and at 36 weeks post-conceptional age.

d Incidence rates of CLD for each definition separately: supplemental oxygen requirement for 28 days after birth/at 36 weeks post-conceptional age.

Besides gestational age, other factors may increase the risk of RDS development. At any given gestational age, incidence of RDS is higher for caesarean section without labour than for vaginal delivery.62 Also after a precipitous delivery the risk is increased. ${ }^{136}$ When corrected for gestational age, the occurrence of RDS is significantly increased in gestational diabetes and in insulin-dependent mothers. ${ }^{218}$ Similarly, overnourished infants who are large for gestational age without evidence of maternal diabetes seem to be at increased risk. ${ }^{192}$ Genetic factors may also play an important role in determining susceptibility to RDS. ${ }^{145}$ Incidence is also increased in male sex, the second-born of twins, Caucasian race when compared to the negroid race, ${ }^{33}$ and in situations of cold stress and asphyxia. ${ }^{136}$ 


\subsubsection{Clinical features}

Signs of RDS indicating difficulty in breathing usually appear within minutes after (preterm) birth. Characteristically, infants are cyanotic in room air with expiratory grunting, tachypnoea, subcostal and intercostal retractions, nasal flaring, and duskiness appearance. ${ }^{107,136}$ The increasing cyanosis is often relatively unresponsive to oxygen administration. ${ }^{136}$ Respiratory rate is well above the normal range of 30 to 60 per minute to maintain adequate alveolar ventilation at low tidal volumes due to increased stiffness of the lung. The usually prominent expiratory grunting is caused by partial closure of the glottis to maintain lung volume and gas exchange during exhalation. ${ }^{107}$ Breath sounds during auscultation may be normal or diminished with a harsh tubular quality, and, on deep inspiration, fine rales may be heard, especially over the lung bases posteriorly. ${ }^{136}$ Blood gas and acid-base values are characterized initially by hypoxemia and later by progressive hypoxemia, hypercarbia, and variable metabolic acidosis. Within 6-12 hours typical radiological abnormalities may develop at the chest radiograph, which can be graded according to a system described by Giedion and colleagues comprising of four stages. It should be noted that modern techniques of mechanical ventilation are likely to obscure these abnormalities. ${ }^{81}$ Stage 1 abnormalities include very fine reticulo-granular pattern, normal lucency, and air bronchograms absent or only present within the cardiac border; stage 2 abnormalities show a generalized typical reticulo-granular pattern, slightly diminished lucency, and air bronchograms extending the cardiac border; stage 3 includes confluent reticulogranular densities, marked reduction of lucency with indistinct cardiac and diaphragmatic borders; and stage 4 represents total opacification with a total white appearance. ${ }^{81}$ Increasingly this classification is thought to be obsolete, especially because early mechanical ventilation is able to obscure the radiographic characteristics. Figure 3 shows an example of the radiological appearance of RDS.

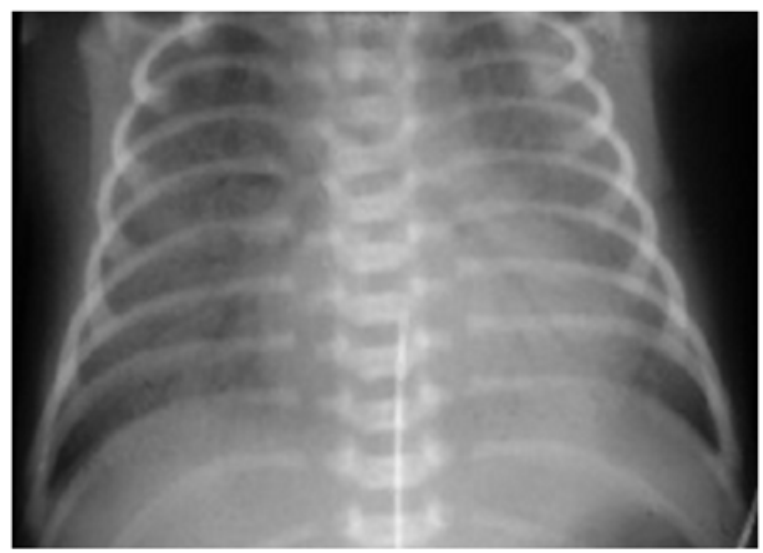

Figure 3.

Radiological appearance of RDS stage 3 according to Giedion. Confluent reticulogranular densities can be recognized with a marked reduction of lucency and indistinct cardiac and diaphragmatic borders. 
The natural course is characterized by progressive worsening of cyanosis and dyspnoea. Initially, the unventilated infant requires 40 to 50 per cent oxygen after birth for relief of central cyanosis, but develops an increasing oxygen requirement over the subsequent 24 to 48 hours, sometimes reaching as high as 100 per cent.107 Progression and duration of RDS depends on the therapeutic measures and complications that may arise during the course of the disease. If RDS is uncomplicated, symptoms and signs may reach a peak within 3 days, after which gradual improvement sets in. Improvement is often heralded by a spontaneous diuresis and the ability to oxygenate the infant with lower inspired oxygen levels. ${ }^{136}$ The decline in oxygen requirement is relatively rapid after 72 hours and, usually, oxygen can be discontinued after 1 week. However, complications during the course of RDS occur frequently. Especially extremely preterm infants often require mechanical ventilation and show a prolonged course of respiratory disease. Complications may include air leaks, like pulmonary interstitial emphysema, pneumothorax, pneumomediastinum, and pneumopericardium; patent ductus arteriosus which often becomes apparent after recovery from RDS; peri- and intraventricular haemorrhage and hypoxic-ischemic brain damage; and chronic lung disease of prematurity (CLD). ${ }^{107}$ Death is rare on the first day of illness, usually occurs between days 2 and 7, and is associated with alveolar air leaks (interstitial emphysema, pneumothorax), and pulmonary or intraventricular haemorrhage. Mortality may be delayed weeks or months if CLD develops in mechanically ventilated infants with severe hyaline membrane disease.

\subsubsection{Pathophysiology}

Although in foetal lung homogenates of 20 weeks gestation surfactant is present at high concentrations, it does not reach the surface of the lung until later. Between 28 and 32 weeks surfactant appears in the amniotic fluid. Mature levels of pulmonary surfactant are usually present after 35 weeks of gestation. Surfactant synthesis depends in part on normal $\mathrm{pH}$, temperature, and perfusion. Asphyxia, hypoxemia, and pulmonary ischaemia, particularly in association with hypovolemia, hypotension, and cold stress, may suppress surfactant synthesis. The epithelial lining of the lung may be injured by high oxygen concentrations and, together with the effects of respiratory management, this may result in further reduction of surfactant. ${ }^{136}$

Due to deficiency of surfactant production, the alveolar surface tension increases producing atelectases in presence of a highly compliant chest wall and small respiratory units. The number of adequately ventilated alveoli is decreased and hypoxia may develop. Decreased lung compliance, small tidal volumes, increased physiologic dead space, increased work of breathing, and insufficient alveolar ventilation eventually result in hypercarbia. ${ }^{136}$ The collagen and elastin matrix is underdeveloped in preterm infants, and the pressure-volume curve for the preterm lung will not achieve a plateau or maximal volume. Increasing inspiratory pressures 
will yield linearly increasing volumes yielding additional lung damage. ${ }^{129}$ Furthermore, following hypercarbia, hypoxia and acidosis, ${ }^{83}$ pulmonary arterial vasoconstriction may develop with increased right-to-left shunting through the oval foramen, arterial duct, and within the lung itself. Because of reduced pulmonary blood flow, ischemic injury to the surfactant producing cells and the vascular endothelial cells, effusion of proteinaceous material, recognizable as hyaline membranes, into the alveolar spaces occurs. Interstitial oedema may develop and, as a consequence, the compliance of the lungs further decreases, requiring higher pressures to expand the small alveoli and airways. ${ }^{136}$ Epithelial damage appears within 30 minutes of the onset of breathing, and the hyaline membranes appear within 3 hours after birth. Bronchiolar lesions may result from a combination of atelectases in terminal air spaces and disruptive overdistention of more proximal airways. ${ }^{107}$ As a consequence of atelectasis, the functional residual capacity (FRC), measured by nitrogen washout, can be reduced. When measured with body plethysmography FRC may be increased, because of airtrapping secondary to bronchiolar obstruction. Although bronchus obstruction may exist, most of the affected bronchi may be collapsed and pulmonary resistance may not be gravely affected in this situation. ${ }^{136}$ (See also Figure 5.) It must be emphasized that severity of RDS has decreased substantially during the past decades and the severe forms of RDS with massive atelectases are rare nowadays, because of improved ventilatory care, i.e. antenatal corticosteroid treatment, more effective ways of mechanical ventilation that are less harmful for lung tissue, and exogenous surfactant therapy.

\subsubsection{Prevention and treatment}

In case preterm labour can no longer be postponed, administration of dexamethasone or betamethasone to women 48 to 72 hours before delivery of foetuses at 32 weeks or less of gestation prevents development of severe RDS and significantly reduces the incidence, morbidity and mortality of RDS up to about 40 per cent. ${ }^{37,129,134}$ However, in case of imminent preterm birth the required time for steroids to become effective is often too short. Postnatally, early selective use of surfactant administered in infants requiring assisted ventilation leads to a decreased risk of neonatal mortality and CLD at 36 weeks gestation in comparison with a delay in such treatment. ${ }^{289}$ Antenatal steroids may act synergistically with postnatal exogenous surfactant therapy. ${ }^{136}$

Treatment is required in presence of an inadequate exchange of oxygen and carbon dioxide with secondary metabolic acidosis and circulatory insufficiency. Early supportive care to minimize acidosis, hypoxia, hypotension, and hypothermia lessen RDS severity. Treatment requires careful and frequent monitoring of heart and respiratory rates, arterial partial oxygen $\left(\mathrm{PaO}_{2}\right)$ and carbon dioxide pressures $\left(\mathrm{PaCO}_{2}\right)$, $\mathrm{pH}$, bicarbonate, electrolytes, blood glucose, haematocrit, blood pressure and temperature. ${ }^{136}$ Warm humidified oxygen should be provided at a concentration 
sufficient to keep the oxygen saturation levels of haemoglobine $\left(\mathrm{SaO}_{2}\right)$ between 90 and 95 per cent. When this cannot be achieved by supplemental oxygen alone, continuous positive airway pressure (CPAP) by nasal prongs is indicated. This usually produces a sharp rise in $\mathrm{PaO}_{2}$. When despite these measures, the inspiratory oxygen fraction $\left(\mathrm{FiO}_{2}\right)$ remains above 0.7 , the partial arterial carbon dioxide pressure exceeds $55 \mathrm{mmHg}$, acidosis persists with $\mathrm{pH}$ decreasing below 7.25 , or serious apnoeas persist, mechanical ventilation is required to achieve adequate $\mathrm{SaO}_{2}$ levels and to keep the $\mathrm{PaCO}_{2}$ between $40-45 \mathrm{mmHg}$. When peak inspiratory pressure during mechanical ventilation (PIP) remains below $20 \mathrm{cmH}_{2} \mathrm{O}$ while $\mathrm{FiO}_{2}$ rises above 0.6, or when PIP exceeds $20 \mathrm{cmH}_{2} \mathrm{O}$ while $\mathrm{FiO}_{2}$ is above 0.45 rescue therapy with exogenous surfactant is required. Rescue treatment is usually initiated in the first 24 hours of life via the endotracheal tube and multiple doses may be needed. ${ }^{136}$

\subsubsection{Factors affecting postnatal lung development: chronic lung disease of prematurity (CLD)}

Despite successful efforts to decrease RDS severity, like exogenous surfactant, increased use of antenatal corticosteroids, more effective modalities of mechanical ventilation that are less harmful for lung tissue, a more careful use of fluid intake and a more aggressive management of a patent ductus arteriosus, the incidence CLD remained high or may even have increased ${ }^{14,55}$ and RDS does not seem to be a prerequisite for CLD development anymore. ${ }^{201}$

\subsubsection{History and epidemiology}

As a consequence of improved survival in preterm infants, proliferate reparative phenomena had a chance to develop, as was described for the first time in 1962 by Boss and colleagues. ${ }^{24}$ This was supported by the findings of Robertson and colleagues in 1964, who found thickened alveolar walls with an increase of fibroblasts and excess of reticulin or collagen fibres, and radiological abnormalities, like emphysematous blebs and patchy infiltrates changing to small areas of atelectases in these infants. ${ }^{219}$ In 1967, Northway and colleagues described the clinical, histological and radiological features of this pulmonary disease following respiratory therapy of RDS and they called it bronchopulmonary dysplasia (BPD). ${ }^{197}$ These infants developed severe RDS for which they required high $\mathrm{FiO} 2$ and prolonged mechanical ventilation with high positive airway pressures. However, as a consequence of decreased RDS severity nowadays and increased survival of extremely premature infants (i.e. 24-26 weeks gestation, birth weight $<1000$ grams), who are most likely to develop CLD, $55,196,203$ the clinical features and definition of CLD has been changing since then. The initial description by Northway and colleagues ${ }^{197}$ was followed by a definition consisting of the presence of respiratory distress, abnormal chest radiograph 
and need for supplemental oxygen after 28 or 30 days of age in 1979. ${ }^{13,259}$ Since need for supplemental oxygen may be a function of immaturity rather than intrinsic lung pathology, Shennan and colleagues proposed a definition that better predicted pulmonary outcome and consisted of the requirement for additional oxygen at 36 weeks corrected post-conceptional age in 1988.230 Both definitions for CLD (i.e. oxygen requirement at 28 days of life and at 36 weeks post-conceptional age) have remained in common use until now, ${ }^{143}$ and it has been proposed that a combination of these definition ${ }^{59}$ more accurately describes the subsequent adverse pulmonary effects of preterm birth, although it is not in widespread use yet. ${ }^{194}$ However, in practice, even the term BPD is still used synonymously with CLD. ${ }^{201}$

Apart from the use of different criteria to define CLD, the reported variation in incidence rates of CLD can be attributed to differences in patient populations and management. ${ }^{55}$ Valuable reported data about incidence rates include information regarding the definition used and the patient population studied. In 1989, the overall percentage of infants with RDS developing CLD (based on oxygen requirement beyond 28 days of age) was similar to the incidence rate of BPD in the early 1960s (31 per cent vs. 28 per cent, respectively) (Table 1), but, simultaneously, the number of infants surviving with birth weights below 1000 grams had dramatically increased. Since the incidence rate of CLD increased between 1987 and 1992 and subsequently decreased between 1992 and 1997, accompanied by a significant improvement of survival, the actual risk of CLD development may have fallen. ${ }^{63,163}$ In The Netherlands, a small decreasing trend of the incidence rate of CLD was seen between 1993 and 2001. However, care should be taken when interpreting these data, since registration in 1993 was not complete.

\subsubsection{Clinical features}

'Classic' BPD, as described by Northway and colleagues, ${ }^{197}$ is a severe form of chronic lung disease that traditionally occurred in infants experiencing RDS or other lung problems after prolonged exposure to high levels of ventilator support and oxygen. ${ }^{128}$ Since this form of chronic lung disease is uncommon nowadays, only the clinical features of the 'new' CLD will be discussed. Although not necessary, CLD still often develops in the setting of neonatal RDS, most often secondary to surfactant deficiency and pulmonary immaturity. ${ }^{201}$ Infants who develop CLD nowadays initially require, in contrast to the classic BPD, only low or moderate concentrations of oxygen, mechanical ventilation with low pressures, and show a favourable response to the administration of surfactant during the acute phase. 55 The modest ventilator and oxygen needs decrease over weeks to months ${ }^{128}$ and chest radiographs appear diffuse hazy and dense, persisting over time and sometimes progressing into a fine lacy parenchymal pattern with modest hyperinflation and a few large cysts (Figure 4). 12,258 Maldistribution of ventilation leads to a mismatch of ventilation and perfusion resulting in hypoxemia. Both respiratory rate and physiologic dead space are increased 


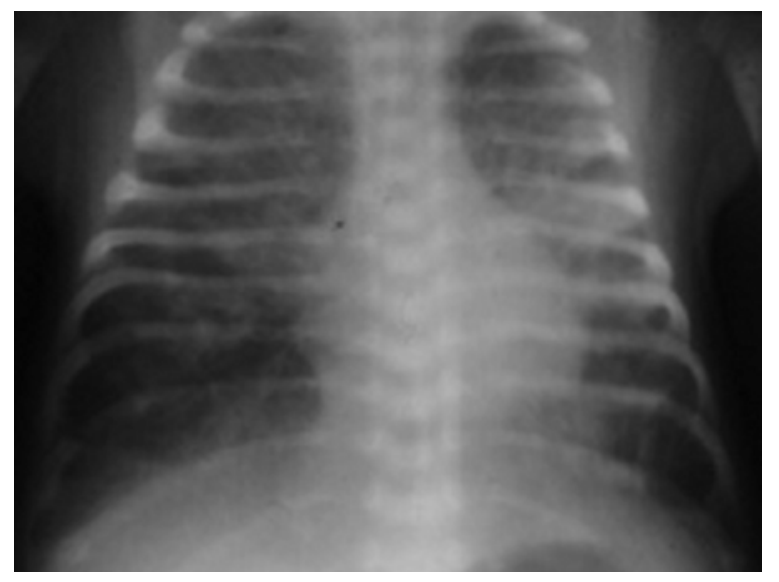

\section{Figure 4.}

Radiological appearance of CLD at 1e month after birth. Small cystic lucencies appear in both lungs alternating with dense areas. Lung volume is increased, presented by the horizontal configuration of the ribs.

causing decreased alveolar ventilation and elevated $\mathrm{PaCO}_{2}$ values. Severity of CLD varies considerably. Infants with minimal disease (the vast majority) show some signs of respiratory insufficiency during exercise, e.g. tachypnoea and hypoxia when feeding, but a large number are asymptomatic and do not require supplemental oxygen by the time they are discharged home. Infants with more severe disease show signs of respiratory insufficiency sufficient to require prolonged oxygen therapy beyond 36 weeks post-conceptional age. Airflow obstruction can be recognized by wheezing, decreased exercise tolerance, insufficient respiratory reserve, deformities of the chest wall (hyperinflation) and a depressed liver. Even in rest, these children may exhibit tachypnoea, hypoxia, and intercostal and subcostal recession. Generally during the first 2 years of life, a gradual improvement in pulmonary function is seen due mainly to continuing lung growth and constant repair and remodelling. However, even those with mild disease are prone to recurrent attacks of wheezing especially in association with viral respiratory tract infections. Infants with moderate to severe CLD may be wheezy even at rest. ${ }^{201}$ Most children are weaned of oxygen within a few months, but a few may require supplemental oxygen for several years..$^{53}$ Since survivors of the 'new' CLD did not reach adult age yet, pulmonary sequelae at this age are unknown to date.

\subsubsection{Pathophysiology}

CLD is a multifactorial disease. Infants who develop CLD nowadays are more immature compared to the 'classic' BPD and signs of RDS are usually mild and may even be absent. ${ }^{31}$ A predominant pathogenetic factor in CLD is the interruption of normal lung parenchymal development during the canalicular or saccular stage of human lung development (paragraph 1.1.1.) at preterm birth, resulting in fewer and 
larger alveoli. The total number of arteries may also be reduced, maintaining a normal alveolar to arterial ratio in infants with CLD, while a marked alveolar hypoplasia may become prominent during subsequent years. 55,104

While the 'classic' BPD was mainly the consequence of barotraumas and oxygen toxicity, the 'new' CLD in the surfactant era results from the interaction of many factors, including extreme prematurity, poor respiratory effort, need for prolonged mechanical ventilation and colonization of the airways with pathogens triggering an inflammatory cascade. ${ }^{14}$ Frequently, these infants develop sepsis and in many of them a patent ductus arteriosus reopens. ${ }^{85}$ Hence, the persistent or recurrent pulmonary inflammatory reaction, although less prominent compared to the 'classic' BPD, ${ }^{129}$ is probably caused by intrauterine infection/inflammatory disease that continues after birth, or by neonatal damage to the airways and airspaces due to hyperoxia, barotrauma/volutrauma or other toxins. ${ }^{292}$ Elevated concentrations of oxygen lead to the production of oxygen radical species and the release of chemotactic factors that attract polymorphonuclear leukocytes to the lung. When activated, these cells release inflammatory mediators and proteolytic enzymes. Compared to infants born at term, preterm infants have significantly lower levels of antioxidant enzymes, such as catalase, superoxide dismutase, and peroxidase and, consequently, are more susceptible to oxygen toxicity. During progression to CLD leukocytes remain present in alveolar lavages ${ }^{129}$ and anti-inflammatory cytokines such as interleukin (IL)-10 are low when pro-inflammatory cytokines are high. ${ }^{130}$ Presence of multiple protein and lipid mediators of inflammation in lung tissue cause a prolonged release of proteolases. Eventually, destruction of elastin by uncontrolled elastase activity due to an imbalance between elastase and alpha protease inhibitor induce development of neonatal lung injury. ${ }^{12,194}$ Consequences of persistent pulmonary inflammation include deficiencies in surfactant components (SP-A), ${ }^{104}$ interstitial and peribronchial oedema, loss of epithelial cells and ciliae, necrotizing bronchiolitis and, eventually, fibrosis. ${ }^{106,201}$ Elevated concentrations of fibronectin in the lung, released by pulmonary alveolar macrophages, and macrophage inflammatory protein-1 are associated with development of pulmonary fibrosis. ${ }^{12}$ Pulmonary oedema before or during CLD development may be exaggerated by reopening of the ductus arteriosus decreasing lung compliance and increasing airway resistance by narrowing of the terminal airways. ${ }^{197}$ Reopening of the ductus may also play a pathogenetic role in the development of CLD. ${ }^{12}$

At autopsy of infants with CLD, pulmonary fibrosis is less compared to the 'classic' BPD, but deregulated alveolarization ${ }^{160}$ or arrested airway development is increased. ${ }^{125}$ Open lung biopsy specimens from infants of low birth weight on ventilator support (postnatal age 2 weeks to 7 months) were reported to show a simplified distal lung acinus, consistent lack of significant alveolarization, abnormal capillary configuration, and an adaptive dysmorphic pattern of vascular organization. ${ }^{106}$ Additionally, normal development of the extracellular matrix around the smaller airways is disrupted with focal, dense and abnormal deposition of 
elastin. ${ }^{160}$ The walls of both large and small conducting airways are thickened largely as the result of an increased smooth muscle mass. Alveolar attachments tethering the airway are remarkably decreased in number, while their thickness is increased. This results in a deformation of the diameter of the airways increasing airway resistance. ${ }^{201}$ Airway resistance further increases by damage and destruction of airways (airway plugging with cellular debris), airway inflammation with peribronchial oedema and increased mucus production, and airway wall thickening by increased airway smooth muscle mass also exhibiting bronchospasms. ${ }^{80,84,106,151,201}$ Increased baseline airway resistance is only partly reversible by inhaled bronchodilators and systemic corticosteroids. ${ }^{201}$ Static compliance is decreased, but may be increased if damage to the lung is sufficient to result in loss of elastic recoil. ${ }^{106}$ FRC may be decreased following diffuse atelectases, but may also be increased in presence of air trapping caused by increased dynamic airway obstruction. As a consequence of alveolar hypoplasia, total lung capacity and lung compliance may be decreased. Infants and children with CLD exhibit bronchial hyperresponsiveness ${ }^{201}$ due to interruption of normal maturational changes in respiratory mechanics as a consequence of impaired lung maturation (paragraph 1.1.3.). ${ }^{180}$ (See also Figure 5.)

\subsubsection{Prevention and treatment}

Preventive measures include limitation of potential provocative factors. As in RDS, prematurity should be prevented whenever possible. The infant's heart rate can be recorded during uterus contractions, reducing the risk of neonatal asphyxia. After birth, supplemental oxygen therapy and high inspiratory pressures during mechanical ventilation should be avoided when possible. Current practice is to maintain $\mathrm{SaO}_{2}$ between 92 and 95 per cent instead of higher values. ${ }^{194} \mathrm{~A}$ few large ventilator breaths during resuscitation may already induce an inflammatory cascade, compromising potential improvement by other interventions, like surfactant therapy..$^{14,129}$ Prophylactic use of nasal continuous positive airway pressure (nCPAP) may preclude initiation of intermittent positive pressure ventilation (IPPV) and may thereby decrease the risk of CLD development, but this was not confirmed by systematic reviews. ${ }^{247}$ Since Kraybill and colleagues found that CLD rates were inversely related to $\mathrm{PaCO}_{2}$ levels, ${ }^{139}$ it was thought that permissive hypercapnia may prevent hyperventilation and, thereby, severe lung damage. However, a clear benefit in reducing mortality, pulmonary and neurodevelopmental morbidity has not been proven yet. ${ }^{284}$ Injury to surfactant-deficient lungs can be minimized by recruiting lung volumes and then maintaining a higher-than-normal FRC by high-frequency oscillatory ventilation (HFOV). ${ }^{72}$ More recently described recruitment strategies, in which pressures are gradually increased to recruit collapsed lung units followed by decreasing pressures to the least PEEP necessary to maintain sufficient alveolar recruitment with or without the use of surfactant, are more promising with regard to decreasing lung injury. ${ }^{217,270}$ 
Diuretics may immediately improve lung mechanics by diminishing pulmonary oedema and, thereby, reducing the need for high inspiratory pressures. ${ }^{25}$ Effective treatment of a patent ductus arteriosus reduces interstitial oedema. Because of increased expenditure of energy probably due to increased work of breathing caloric intake should be elevated. Growth is extremely important to overcome respiratory problems associated with preterm birth, because airway resistance decreases (Figure 2) and the response to bronchodilators and corticosteroids improves with somatic growth. ${ }^{201}$ Other measures include vigilance regarding antenatal and postnatal infections, including postnatal sepsis. Prevention of prematurity should get the highest priority, but prolonged exposure of the foetus to pro-inflammatory cytokines may inadvertently promote development of CLD. Therefore, strategies to identify important antenatal cytokines related to CLD development may increase future potential for prevention. ${ }^{128}$ Although antenatal administration of corticosteroids improves lung maturation and decreases the incidence of RDS, repeated courses may increase the incidence of CLD. ${ }^{70}$

Postnatal corticosteroids facilitate extubation from the ventilator due to the antiinflammatory effect ${ }^{48}$ reducing airway oedema and obstruction. ${ }^{42}$ However, because of the adverse long-term side effects, ${ }^{97,266}$ its use has been restricted dramatically during recent years. In some centres, postnatal corticosteroids are not being administered at all. In our centre, hydrocortisone instead of dexamethasone is used. There may be indications that some of the adverse long-term side effects are less severe when hydrocortisone is used. ${ }^{268}$ Alternatively, in some infants, bronchodilators help to decrease airway resistance in $\mathrm{CLD}^{282}$ and inhaled corticosteroids may accelerate extubation. ${ }^{153}$

One of the innovative treatments is nitric oxide that may be efficient in the treatment of severe hypoxemic RDS. ${ }^{16}$ As long as the randomized controlled trial testing the hypothesis that early low-dose inhaled nitric oxide may reduce the incidence of death or CLD in infants with severe RDS is under way, nitric oxide therapy should be considered an experimental drug, and its use restricted to clinical studies in this specific population. ${ }^{217}$

After discharge from hospital, managing hypoxemia with low flow domiciliary oxygen and nutrition has improved the respiratory outcome of children with CLD. 53 Nutrition has been improved with calorie and vitamin supplementation orally or, when necessary, given via a naso-gastric tube or a percutaneous gastrostomy. Drug therapy includes inhaled corticosteroids and, if associated with symptomatic improvement, beta-2-agonist bronchodilators. Oral corticosteroids may be used during respiratory exacerbations. Diuretics may be helpful when there is evidence of right heart involvement with interstitial pulmonary oedema. Immunizations against respiratory pathogens Haemophilus influenzae type B, Bordetella pertussis, and influenza viruses are given at the earliest opportunity. Specific anti-respiratory syncytial virus 
(anti-RSV) immunoglobulin therapy during winter months has recently become common practice in the European population. ${ }^{255}$

\subsubsection{Factors affecting postnatal lung development: postnatal therapeutic interventions}

Although life-saving, mechanical ventilation induces additional lung injury when the lungs are inflated to volumes that exceed total lung capacity. Immature lungs are especially susceptible to lung damage, because only 10 to $20 \mathrm{ml} / \mathrm{kg}$ of lung gas volume is available between FRC and total lung capacity for safe ventilation of the preterm infants without hyperinflation. ${ }^{129}$ Regional or uniform overinflation causes rupture of the distal air spaces leading to pulmonary interstitial emphysema and leukocyte migration (macrophages and granulocytes) into lung parenchyma inducing an inflammatory cascade with increased permeability, and interstitial and alveolar

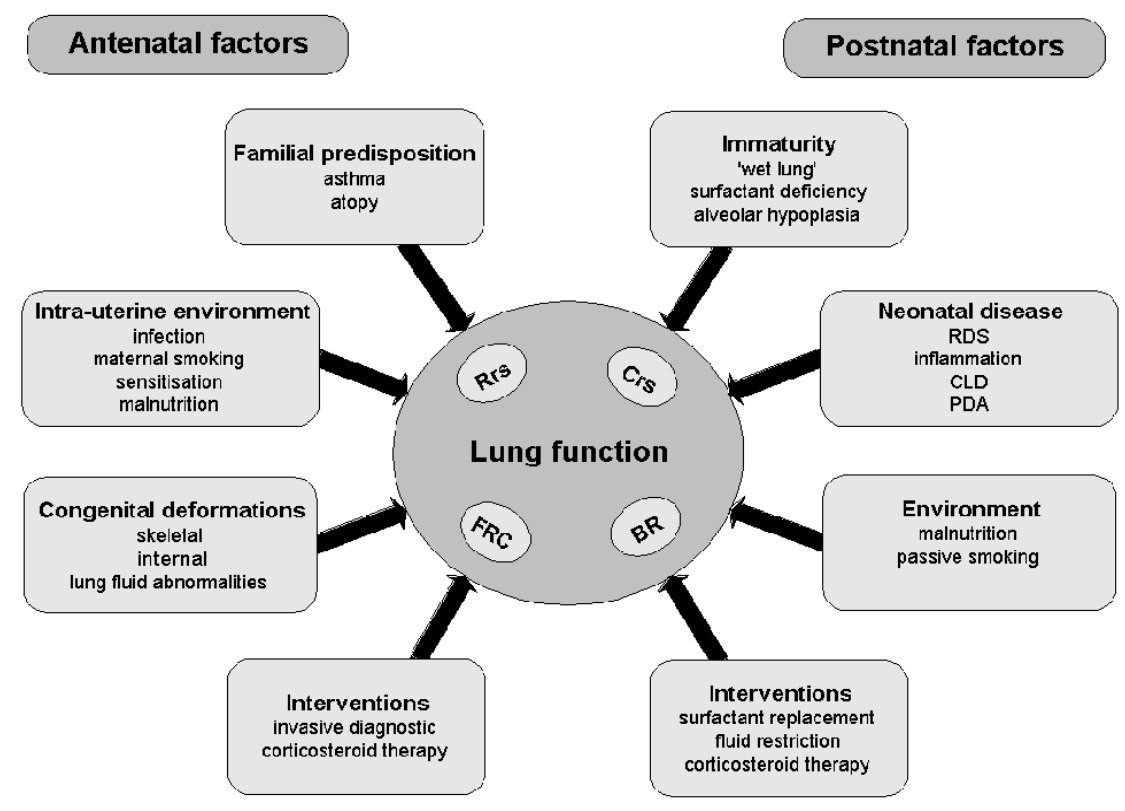

Figure 5. Summary of ante- and postnatal factors affecting postnatal lung function. $\operatorname{Rrs}=$ respiratory system resistance; $C r s=$ respiratory system compliance; $\mathrm{FRC}=$ functional residual capacity. $\mathrm{BR}=$ bronchial responsiveness. 
oedema. ${ }^{14,129,198}$ As with overdistention, ventilation at low lung volumes promotes accumulation and activation of peripheral leukocytes in the lungs, because of the cyclic opening and closing of lung units. Mechanical ventilation seems to interfere not only with alveolarization but also with the surfactant system, causing increased lipid pools in tissue, while alveolar pools stayed relatively low at about $20 \mathrm{mmol} / \mathrm{kg}$ of saturated phosphatidylcholine. Already after a few large ventilator breaths, secretion of de novo-synthesized saturated phosphatidylcholine may be decreased and its catabolism increased. ${ }^{14,129}$ Important preventive measures regarding mechanical ventilation have to focus on prevention of high inspiratory pressures especially during acute resuscitation, gradual recruitment of collapsed lung units, maintaining sufficient alveolar recruitment and minimizing supplemental oxygen to preclude an stimulation of the pulmonary inflammation. ${ }^{194}$

Surfactant treatments have profound effects on static lung volumes that translate into better lung mechanics, improved gas exchange, and a 30 to 40 per cent decrease in mortality for preterm infants with RDS. Oxygenation is improved by increased recruitment of new gas exchange surface as well as by increasing functional residual capacity (FRC) due to increased stability of the lungs on deflation (Figure 5)..129 Immediate effects include improved alveolar-arterial oxygen gradients, reduced ventilator mean airway pressure, ${ }^{124}$ increased pulmonary compliance (Figure 5), 45,113 and improved appearance of the chest roentgenogram..$^{50}$ By stabilizing lung volume and avoiding alveolar overdistention surfactant may mitigate the inflammatory response in the lung. In addition, endotoxin-stimulated secretion of several proinflammatory cytokines, like tumour necrosis factor, IL-1 and IL-6 by alveolar macrophages, may be decreased after administration of surfactant. ${ }^{256}$ Hence, surfactant treatment may decrease the granulocyte accumulation and oedema that is partly caused by mechanical ventilation. ${ }^{129}$ Furthermore, surfactant also inhibits neutrophil superoxide production ${ }^{5}$ and has been shown to reduce bacterial growth in experimentally induced group-B streptococcal infection in immature rabbits. ${ }^{114}$ On the other hand, administration of surfactant increases the risk of overdistention of some air spaces if peak inspiratory pressures are not reduced as rapidly as lung compliance improves. ${ }^{14}$

The rationale for the postnatal use of glucocorticoids was primarily to prevent or treat CLD by minimizing the inflammation and injury associated with ventilation and oxygen exposure. By suppressing a number of indicators of inflammation/injury, postnatal glucocorticoids acutely improve lung function, facilitate extubation, but only have modest effects on the incidence of CLD defined as supplemental oxygen need at 36 weeks. ${ }^{96,101,103}$ Unfortunately, according to experiments in rodents, the major effect of glucocorticoids on the pre-alveolarized postnatal lung is to decrease alveolar and vascular development. ${ }^{164}$ Furthermore, postnatal corticosteroid therapy increases the risk of adverse long-term neurodevelopmental outcome. ${ }^{95,266}$ As a consequence of these recent publications, the use of postnatal corticosteroid therapy has been 
dramatically restricted. The Cochrane Reviews regarding this kind of therapy recommend to limit the use of postnatal corticosteroids to infants, who cannot be weaned from the ventilator, and to perform further research to find out safer treatment strategies and the effectiveness of less potent drugs. ${ }^{94,100,103}$

Diuretics diminish interstitial and peribronchial oedema, theoretically improving lung function (Figure 5) and, consequently, decreasing ventilator conditions. Inhaled corticosteroids will limit the ongoing inflammatory processes, reducing pulmonary oedema and potentially decreasing bronchial responsiveness, although the relationship between pulmonary inflammation and the subsequent development of bronchial hyperresponsiveness is still obscure.201 Bronchodilators will reduce the reversible component of airway narrowing, if present, directly decreasing airway resistance (Figure 5). Stimulating somatic growth by sufficient calorie and vitamin intake will decrease airway resistance, increase lung volume (Figures 2 and 5) and improve the response to inhaled bronchodilators and corticosteroids although in the more severe cases the irreversibility may be permanent. 


\section{CHAPTER 2}

Respiratory function at neonatal age and during the first years of life 

After birth the lung starts to function as an absorptive organ in contrast to its secretory function in utero. The primary objective of the lung is to provide the body with oxygen $\left(\mathrm{O}_{2}\right)$ and to release carbon dioxide $\left(\mathrm{CO}_{2}\right)$ that is produced by metabolism processes. This is achieved by:

1. Ventilation of the lung by environmental air.

2. Gas exchange at the alveolar surface area by diffusion of $\mathrm{O}_{2}$ from the alveolar space into the capillary system and of $\mathrm{CO}_{2}$ from the capillary system into the alveolar space.

To accomplish this, the respiratory system consists of active components including (a) respiratory muscles which generate pressures required to produce airflow between alveoli and mouth and (b) smooth muscle in the airway walls regulating resistance to flow, and passive components including the airways, their diameter depending on lung volume, and the gas exchange surfaces of the alveoli. All components may be affected by age and respiratory disease. In this chapter, respiratory function will be discussed starting with the physiological properties of the respiratory system followed by a description of various possibilities to measure respiratory function in infancy and early childhood. At the end of the chapter, the tests that were used for the studies in this thesis are described in detail.

\subsection{Physical properties of the respiratory system}

\subsubsection{Forces operating during quiet ventilation}

Although, already in the second century, Galen described the mechanism of breathing as the expansion of the thorax allowing the weight of the surrounding air to fill the increased volume, ${ }^{245}$ it took until 1820 to complete the description of forces involved in breathing by a description of lung elasticity. ${ }^{28}$ This was confirmed by Donders in 184952 and developed further by Rohrer in $1916{ }^{208}$ who described the pressure-volume relationships of the lungs and chest wall, including anatomical chest wall, diaphragm and abdominal contents. To produce inspiratory flow, the intrapulmonary pressure has to become subatmospheric. To achieve this, inspiratory muscles, mainly diaphragm, produce a pressure that is more negative than the recoil pressure of the lung tending to collapse it. Airflow is directed into the lungs according to Boyle's/Mariotte's law, stating that the product of pressure and volume is constant for an isothermal process. For an adequate expiration, relaxation of inspiratory muscles is required. The energy stored in the lung, its elastic recoil, is sufficient to generate expiratory flow. ${ }^{123}$ During the respiratory cycle, several pressures operate at different locations inside the thorax that can be expressed as differences relative to barometric pressure $\left(P_{\mathrm{B}}\right)$ (Figure 1). 


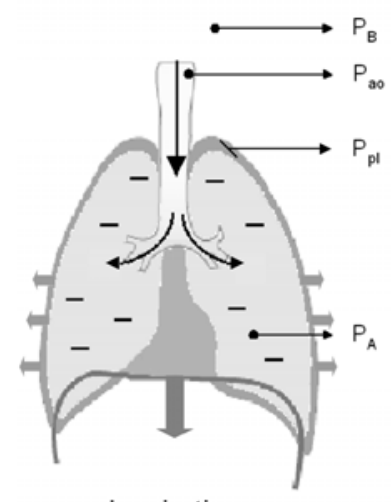

Inspiration

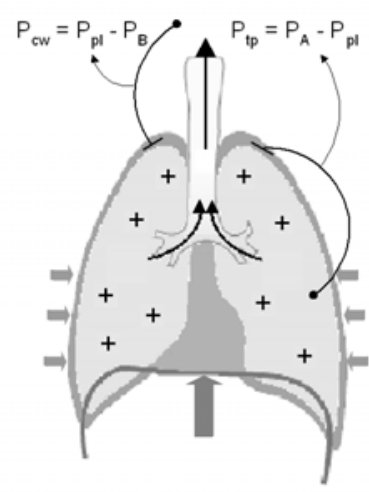

Expiration

Figure 1. Pressures and pressure differences in the respiratory system, while presenting the intrapulmonary situation during in- and expiration.

At static conditions, when no flow is present, it is assumed that the pressure measured at the airway opening $\left(P_{\mathrm{ao}}\right)$ resembles alveolar pressure $\left(P_{\mathrm{A}}\right)$. This pressure can be used to assess mechanical properties of the respiratory system, which can be separated into lung and chest wall components. The lung component or transpulmonary pressure $\left(P_{\mathrm{tp}}\right)$ is the difference between $P_{\mathrm{A}}$ and the interpleural pressure $\left(P_{\mathrm{pl}}\right)$, which is usually measured in the oesophagus. The chest wall component $\left(P_{\mathrm{cw}}\right)$ is the difference between $P_{\mathrm{pl}}$ and $P_{\mathrm{B}}$. Actual pressure course of alveolar and pleural pressures during the entire respiratory cycle is presented in Figure 2.

The pressure differences can be used to estimate mechanical characteristics of the respiratory system. Rohrer applied Newton's laws of motion to the respiratory system, 207 being further developed by his pupil Wirz, who initiated together with his colleague Neergard studies of lung mechanics in humans. ${ }^{184}$ Rohrer proposed three independent forces involved in lung movement, namely, elastic recoil, resistance, and inertance (the force required to initiate movement of the lung mass). According to the equation of motion, the sum of these forces provides the total driving force for breathing:

$$
P_{\text {tot }}=P_{\mathrm{el}}+P_{\mathrm{res}}+P_{\mathrm{in}}
$$

Equation 1

where $P_{\text {tot }}$ is the total pressure to move the lungs, $P_{\mathrm{el}}$ is the pressure required to overcome lung elasticity, $P_{\text {res }}$ is the pressure to overcome airway resistance, and $P_{\text {in }}$ is 


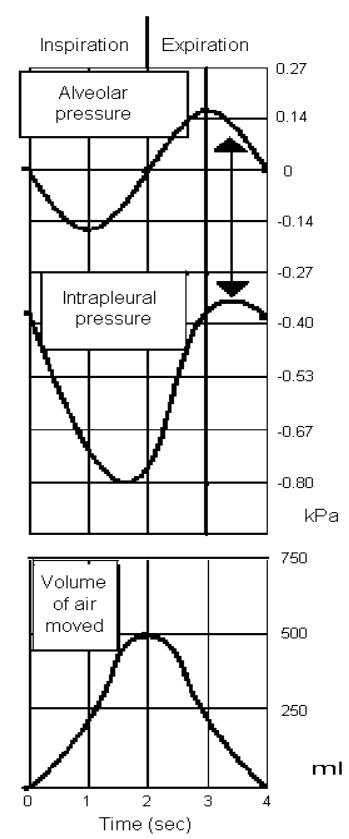

Figure 2. Comparison of changes in alveolar and interpleural pressure during the respiratory cycle. Note that the interpleural pressure normally remains negative throughout the respiratory cycle. By contrast, alveolar pressure is atmospheric when there is no gas flow, that is, at end-expiration and at end-inspiration, but becomes negative during inspiration and positive during expiration, thereby providing the driving force to move air in and out of the lungs. (See also Figure 1.)

the pressure needed to accelerate lung mass. In a situation of zero airflow with complete equilibration of mouth and alveolar pressures and no muscle activity, pressure differences only represent the elastic properties of both lungs and chest wall. $P_{\mathrm{el}}$ is determined by the following components:

$$
P_{\mathrm{el}}=P_{\mathrm{el}}(\text { lung })+P_{\mathrm{el}}(\text { chest wall) }
$$

Equation 2

of which the elastic forces of the lung can be broken down into the surface forces of the alveolar air-liquid interface, responsible for about 65 to 75 per cent of the lung's elastic recoil pressure, ${ }^{123}$ and the retractile forces of the lung tissues. ${ }^{171}$ Increased surface tension at the air-liquid interface in alveoli due to lack of surfactant may therefore substantially increase the pressure required to inflate the lung. This can be explained by LaPlace's law, in which internal pressure of a spherical structure increases with increasing surface tension and decreasing radius. ${ }^{258}$ The physical properties of surfactant include stabilizing the lung by decreasing surface tension relative to the surface area of the alveolus. The larger the surface area, the greater surface tension is allowed before alveoli tend to collapse. So, increased surface tension in a large alveolus offsets the larger radius, making the recoil pressure equal to that in a smaller alveolus. ${ }^{123}$ Elastance of the respiratory system $\left(E_{\mathrm{rs}}\right)$ is further related to volume 
changes, i.e. $P_{\mathrm{el}}$ depends on the volume of air present inside the lungs. Hence, $E_{\mathrm{rs}}$ resembles $P_{\mathrm{el}} / V$.

The resistive component of the driving pressure mainly depends on diameter and geometric configuration of bronchi through which the air is flowing. In infants, peripheral airways may account for a significant proportion of the total resistance to airflow, whereas in older children and adolescents, they account for a negligible proportion of the total resistance of airflow, which is then mainly determined by the central airways:

$$
P_{\text {res }}=P_{\text {res }}(\text { airways })+P_{\text {res }}(\text { lung tissues })+P_{\text {res }} \text { (chest wall) Equation } 3
$$

In the infant, who often breathes through the nose, an additional category due to nasal resistance should be added. ${ }^{230}$ Furthermore, the resistance of the respiratory system $\left(R_{\mathrm{rs}}\right)$ also depends on the physical properties of the air according to Poiseuille's law, in which resistance increases with increasing viscosity of air. ${ }^{21}$ Airway diameter may vary enormously as a consequence of smooth muscle contraction. Also according to Poiseuille's law, $R_{\mathrm{rs}}$ is further related to the rate of volume change (flow: $V$ ), i.e. $P_{\text {res }}$ depends on airflow into or out of the lungs. Hence, $R_{\mathrm{rs}}$ resembles $P_{\text {res }} / V$.

$P_{\text {in }}$ is determined by the following components:

$$
P_{\text {in }}=P_{\text {in }}(\text { lung })+P_{\text {in }}(\text { chest wall })
$$

Equation 4

The inertance of the respiratory system $\left(I_{\mathrm{rs}}\right)$ is related to the rate of flow, i.e. $P_{\text {in }}$ depends on acceleration of volume flowing into or out of the lungs ( $V$ '). Hence, $I_{\mathrm{rs}}$ resembles $P_{\text {in }} / V^{\prime \prime}$.

After replacing these pressure differences by respiratory system elastance, resistance and inertance Equation 1 can be rewritten into Equation 5:

$$
P_{\mathrm{rs}}=E_{\mathrm{rs}} \cdot V+R_{\mathrm{rs}} \cdot V^{\prime}+I_{\mathrm{rs}} \cdot V^{\prime \prime}
$$

Equation 5

$E_{\mathrm{rs}}$ is the reciprocal of compliance $\left(C_{\mathrm{rs}}\right)$, a measure of respiratory system stiffness, and can be replaced by $1 / C_{\mathrm{rs}}$ in Equation 5 . In young infants, the compliance of the chest wall is very high, at least three times as high as that of the lung, increasing to five times as high in preterm infants. ${ }^{84}$ So, at young age, lung compliance is the major factor determining $C_{\mathrm{rs}}$. The principal assumption when applying Equation 5 is that the respiratory system behaves like a single unit and that its mechanical characteristics are linear. ${ }^{220}$ Depending on a single compliance and resistance, the single-compartment respiratory system empties according to a single time constant $(\tau)$, i.e. the time required to expire 63 per cent of the residual air left inside the lungs. Since three time constants are required to achieve 95 per cent lung emptying, the combination of a relatively rapid respiratory rate (short expiratory time) and a long time constant is 
likely to result in dynamic elevation of end-expiratory lung volume and the development of intrinsic positive end-expiratory pressure (iPEEP). The $\tau$ of the respiratory system $\left(\tau_{\mathrm{rs}}\right)$ can be defined as:

$$
T_{\mathrm{rs}}=C_{\mathrm{rs}} \cdot R_{\mathrm{rs}}
$$

Equation 6

Inertial forces are negligible during quiet tidal breathing compared to the much greater elastic and resistive forces. ${ }^{170}$ Pressure-volume relationships of the lungs are assumed to be linear within the tidal volume range and static hysteresis is minimal. ${ }^{184}$ Hysteresis develops in elastic systems at volumes outside the tidal volume range when more pressure is required to move or stretch the system than is recovered on relaxation. Consequently, the pressure-volume curve differs during inspiration and expiration, i.e. at the same volume, $P_{\text {tp }}$ is smaller during expiration than during inspiration.

\subsubsection{Changes in normal respiratory physiology}

Whereas the normal lung may act as a single-compartment model, the associated assumptions tend to break down under conditions of increased ventilator requirements such as exercise, disease, and infancy. At extremes of flow, for instance, resistance becomes non-linear as airflow within the airways moves from a rather predictable laminar flow to turbulent flow. Compliance varies non-linearly at volumes

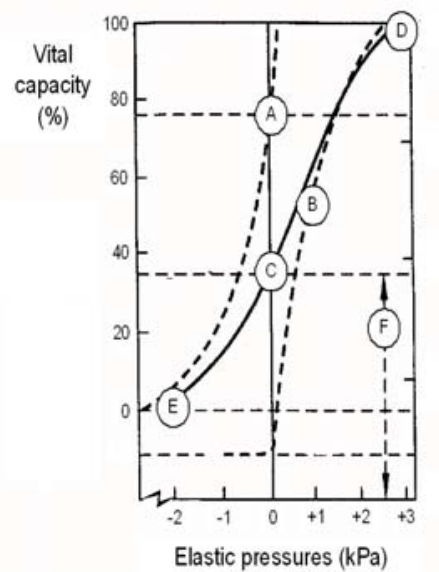

Figure 3. Static volume-pressure curve of the lung, chest wall, and the total respiratory system. Volume is expressed as percentage of vital capacity. The compliance of the lung, chest wall and the total respiratory system are the slopes of the respective curves at the level of the functional residual capacity.

A = chest wall;
B = lung;
C = respiratory system;
D = forced inspiration;
E = forced expiration;
F = functional residual capacity (FRC) 


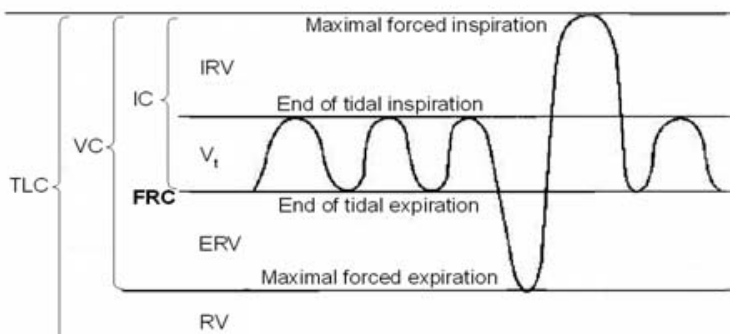

Figure 4. Lung volumes and capacities at different levels during respiration.

TLC = total lung capacity; VC = vital capacity; IC = inspiratory capacity; FRC = functional residual capacity (>elastic equilibrium volume in infants); IRV = inspiratory reserve volume; $V_{\mathrm{t}}=$ tidal volume; $\mathrm{ERV}=$ expiratory reserve volume; $\mathrm{RV}=$ residual volume.

above or below tidal volume (Figure 3). Furthermore, during severe respiratory diseases inequality of ventilation and unequal distribution of resistance and compliance may create a number of single-compartment models, arranged either in parallel to each other or in series. Asynchronous filling and emptying of lung units can result in an important frequency dependence, which can be attributed to continuing gas flows within the lungs while flow at the airway opening has already stopped. Eventually, mechanical models will become increasingly complex and difficult to follow. ${ }^{233}$

Since lung volumes increase dramatically through infancy, childhood, and into adolescence, the distinction between anatomical/morphological growth and functional development varies with age. The chest wall has a major influence on the underlying lungs, as its outward recoil is extremely low in the infant and young child, ${ }^{84}$ but increases with age. The equilibrium of the outward recoil pressure of the chest wall and the inward recoil pressure of the lungs determines the static resting volume of the lungs, i.e. elastic equilibrium volume (EEV). Because of the high compliance of the chest wall in young infants, the respiratory system reaches this equilibrium at a relatively lower lung volume compared to older children and adults. Infants are at risk of airway closure at these low lung volumes, because of decreased distending pressures across the airways. Since this may lead to progressive atelectases and increased venous admixture, end-expiratory lung volume (functional residual capacity (FRC); Figure 4) is dynamically elevated above EEV in young infants to overcome the tendency of the lung to collapse by using various strategies: ${ }^{139,180}$ 
1. Inspiratory muscle activity slowly decreases during the first part of expiration, causing expiratory braking.

2. Subsequent inspiration is initiated prematurely before expiration has reached EEV.

3. Increasing airflow resistance in the upper airways by laryngeal adduction during expiration, further increasing expiratory braking.

4. Increasing respiratory rate with insufficient expiratory time to reach EEV regarding to what is needed with the prevailing respiratory system time constant (see later in paragraph).

Between 6 to 12 months of age, infants abandon these strategies and expiratory flow changes into a more relaxed pattern..$^{35}$

The large central airways are supported by cartilage to resist collapse during expiration, whereas smaller peripheral airways have no such support and rely on a transpulmonary distending pressure together with the surface-tension-reducing properties of surfactant, to remain patent. ${ }^{233}$ Consequently, with a highly compliant chest wall, young infants are very susceptible to peripheral airway closure. Since the peripheral airways are the most substantial part of total respiratory system resistance, peripheral airway closure can have severe consequences, in which mainly basal ventilation is reduced while perfusion is increased, resulting in a significant ventilationperfusion mismatch. ${ }^{40}$ The ability of young infants to cope with added respiratory load is also reduced by the configuration of the respiratory pump. Ribs are placed horizontally, reducing the potential for thorax expansion ${ }^{191}$ and relying more on diaphragmatic activity to increase thoracic volume. Together with the high compliance of the chest wall, inspiratory activity may cause an inward movement of the floppy chest, so that up to about one third of the tidal volume is lost due to paradoxical chest movement. ${ }^{182}$ The loss is made up by an increased work of breathing, even under normal conditions. Furthermore, young infants have fewer type I muscle fibres and are therefore at greater risk of developing respiratory failure due to fatigue. ${ }^{182}$ The high compliance of both airways and chest wall increases the tendency of the airways to collapse at low lung volumes in these infants. Airway smooth muscle is also less contractile and less sensitive to neural and mediator control compared to the mature subject. 229

Sleep state may also affect respiratory function. In the neonatal period, three sleep states can be recognized: active, quiet, and indeterminate (transitional) sleep. ${ }^{39}$ After 6 months of age, sleep states start to resemble those in adults with an active (rapid eye movement) REM sleep for 25 per cent of sleeping time. In preterm infants, REM sleep accounts for 80 per cent of sleeping time and in term infants for 50 per cent. ${ }^{36}$ During REM sleep, diaphragmatic and postural (intercostal and abdominal) muscle tone and laryngeal braking are diminished. As a consequence, FRC (Figure 4) falls during REM sleep. Other circumstances in which lung volumes may be functionally decreased are atelectases, pneumothorax and congenital myopathy. 
Structural reduction of lung volume may occur in cases of lung hypoplasia and alveolar hypoplasia.

Finally, factors affecting $\mathrm{O}_{2}$ uptake will be discussed. Since achieving and maintaining gas exchange is the primary function of the lung, blood gases may complete the picture of total respiratory function. $\mathrm{O}_{2}$ uptake depends on the matching between alveolar ventilation and pulmonary blood flow ( $V^{\prime} / Q^{\prime}$ matching) and on diffusion time, diffusion gradient, and diffusion area. It is not possible to distinguish between different variations of $V / Q$ ' mismatching, for example, whether mainly unventilated areas are well perfused, well-ventilated areas are badly perfused, or inequalities between ventilation and perfusion exist. ${ }^{259} \mathrm{~V} / Q^{\prime}$ matching and diffusion area are substantially affected by lung volume. Both high and very low lung volumes will decrease lung compliance and may impair both alveolar ventilation and effective pulmonary blood flow. During mechanical ventilation, $\mathrm{O}_{2}$ uptake depends mainly on mean airway pressure. The reason is unclear, but it is likely that it is related to optimizing lung volumes and $V / Q$ matching by, for example, recruitment of unventilated alveoli. ${ }^{26}$

Since $\mathrm{CO}_{2}$ diffuses readily from the blood into the alveoli, its elimination depends almost exclusively on alveolar ventilation, which is the product of breathing rate and tidal volume. If the time allowed for inspiration becomes too short and minute ventilation will drop, $\mathrm{CO}_{2}$ retention will occur. ${ }^{65}$ If the time for expiration becomes too short, gas trapping may result, which will manifest as $\mathrm{CO}_{2}$ retention, impaired venous return to the heart, poor cardiac output, and thus compromized $\mathrm{O}_{2}$ uptake. ${ }^{27}$

\subsection{Measuring respiratory function in infants and young children}

Since the 1700s, various attempts have been made to measure lung volumes; the water spirometer has existed for nearly 150 years. ${ }^{121}$ But it was not until the 1940s and 1950s that pulmonary function testing in adults and older children began to develop as a science with clinical, epidemiological, and research applications. ${ }^{3}$ However, tests for measuring lung function in infants have lagged behind. There are a number of reasons that may explain this: (a) infants are uncooperative and therefore difficult to study, (b) sedation is often required and the duration of test procedures is long, (c) parental concerns exist over testing and sedation, (d) lung dynamics may change rapidly, and (e) there may be a lack of physicians and scientists interested in this field. ${ }^{233}$ Although there were pulmonary physiologic studies of normal neonates and new-borns with HMD as early as the mid-1950s ${ }^{2}$ and of infants with bronchiolitis ${ }^{143}$ and cystic fibrosis 198 in the 1960s, the interest in studying the respiratory system in infants has only increased substantially since the past 25 years. Initial tests of lung function in infants focused predominantly on measurements of dynamic compliance, resistance, and lung volumes. ${ }^{2}$ Initially, measurements of the former two were invasive and 
cumbersome, requiring oesophageal balloons or complicated equipment and testing procedures. Recently, technologic advances provided tests that are less invasive and easier to perform, such as passive deflation curves or occlusion techniques, ${ }^{150,180,190}$ the weighed spirometry for measuring respiratory system compliance, ${ }^{44,236}$ forced expiratory manoeuvres by chest compression techniques. ${ }^{234}$ More recently, even less invasive tests such as the analysis of tidal breathing pattern have been devised to assess respiratory health and disease. ${ }^{252}$

In infant lung function testing the optimal measurement condition depends very much on the environment. To optimize success rate, infants should be well fed, clean and dry and placed in a standardized position. Maybe even more important are the qualities of investigators in the field of infant lung function testing, who should be very patient and have great attention to detail. When sedation is allowed in combination with the respiratory function tests, the chance of obtaining reliable repeated measurements is increased..$^{82}$

Mechanical ventilation induces several limitations for respiratory function testing. 228 In ventilated infants, non-linearity of the pressure-volume relationships due to alveolar collapse toward end-expiration, if insufficient positive end-expiratory pressure (PEEP) is used, or overdistension of some lung units toward end-inspiration, if peak ventilation pressures are too high, may substantially affect respiratory function. ${ }^{41}$ Presence of the endotracheal tube will add a (non-linear) resistance to the actual resistance of the airways or respiratory system. ${ }^{152,164}$ To reduce this effect, routine suctioning, to clear secretions in the endotracheal tube and trachea before respiratory function testing, is required. ${ }^{220}$ In infants, endotracheal tubes are uncuffed, increasing the risk of air leakage between tube and trachea. Expiration into a ventilator system with maintained PEEP will affect respiratory function results, as will tube geometry and characteristics of the ventilator tubes connected to measuring equipment. ${ }^{142}$ Characteristics of the inspired gas regarding composition, temperature, relative humidity and pressure may considerably affect respiratory function measurements, ${ }^{21,47,69,92,96,97,110,212,248,250,263,269}$ as does variation in flow rates (Chapter 3 of this thesis). ${ }^{67,73}$ It should be noted that respiratory function testing should be performed during adequately long periods of spontaneous breathing or continuous mechanical ventilation. Combination of the two modes of ventilation (i.e. spontaneous and continuous) causes havoc with analysis and interpretation of the data. One advantage of mechanical ventilation is that airflows and pressures during breathing can easily be recorded via the endotracheal tube; infants and young children often do not accept a facemask that is sealed against their face. Furthermore, results are not affected by the compliance of the upper respiratory tract.

Always note that the equipment used for respiratory function measurements will increase total measured resistance and dead space affecting $\mathrm{SaO}_{2}$ and $\mathrm{PaCO}_{2}$ values, spontaneous breathing pattern and FRC. ${ }^{220}$ 


\section{Respiratory function in early life}

In the following sections, only the respiratory function techniques that were used for the studies in this thesis are described. Considerations regarding the choice of respiratory function measurements are described followed by a description of the equipment required, basic principles, and advantages and disadvantages of the techniques will be discussed.

\subsubsection{Considerations regarding the choice of respiratory function measurements}

The results in this thesis are based on a study population of preterm infants who were mechanically ventilated within 24 hours after birth. Respiratory function measurements were performed in a period ranging from the first day after birth until the second birthday. Considering this group of children, high demands were made upon respiratory function testing. Since vital functions are relatively unstable in preterm ventilated infants, even minimal disturbances can result in severe clinical deterioration. So, measurements should preferably not disturb the patient, be noninvasive, easy applicable and little time consuming. Furthermore, additional dead space and resistance of the equipment should be minimal because of small lung volumes and low muscle strength. When not sufficiently adjusted, these factors may result in inadequate ventilation with increased $\mathrm{PaCO}_{2}$ levels and, eventually, decreased $\mathrm{PaO}_{2}$ levels. From the ethical point of view, it is no topic of discussion that infants should remain inside the incubator and ventilator conditions should not be changed when respiratory function tests are performed. Also, transport of the infants may substantially disturb the patient and is therefore not preferred.

Taking all this into consideration, we chose to use the single breath occlusion technique (SBT) for respiratory function testing during the neonatal period of mechanically ventilated preterm infants. This technique can be easily performed during mechanical ventilation. It only takes a few seconds to put a pneumotachometer with valve mechanism between endotracheal tube and ventilator tubes. Pneumotachometers with a minimal dead space and low resistance to airflow are commercially available. Five occluded breaths are required according to the European Respiratory Society recommendations. ${ }^{80}$ Including the time for the FRC level to regress to original values between separate occluded breaths, it only takes a few minutes to complete the test. When (a) the test is scheduled after a routine suction procedure to remove mucus that may have been accumulated inside the endotracheal tube and (b) testing equipment is situated next to the incubator at the neonatal intensive care unit (NICU), disturbance to the patient will be minimized. This technique is little time consuming. In contrast to the measurement of dynamic respiratory system mechanics instability of the chest wall may be an advantage rather than a disadvantage, because the portion reflected by the lungs will be increased. Compared to the multiple occlusion technique (MOT), this technique less depends on stability of the FRC level, which may substantially vary in preterm infants. In contrast 
to tidal breathing parameters, which can be determined very easy and fast as well, the SBT allows separation of the resistive and elastic properties of the respiratory system. Compared to forced expiration techniques, SBT allows maintaining a PEEP, which is highly recommended in ventilated preterm infants, and infants do not have to be paralysed. Although body plethysmography has the advantage of assessing lung volumes together with mechanics, it probably is too much of an effort for preterm infants to breathe against a closed valve, losing the effect of PEEP. Furthermore, body plethysmography requires expensive equipment, is complicated by pressure and temperature instability, and is rather time consuming. Although lung volume measurements by gas dilution techniques are preferred during mechanical ventilation in preterm infants, measurement errors will be relatively large because of the small lung volumes.

During follow-up period of our study, respiratory function measurements were repeated at 1 year and 2 years of age. At 1 year of age, respiratory function testing can be performed successfully after sedation by chloral hydrate. Since lung volume assessment may add important information to respiratory system mechanics, FRC assessment was added to the measurement of the compliance $\left(C_{\mathrm{rs}}\right)$ and resistance $\left(R_{\mathrm{rs}}\right)$ of the respiratory system, provided that the child was sleeping long enough. Since equipment required to perform the helium dilution technique in these infants was already available, including an appropriate gas analyser and spirometer, this method was chosen.

According to our experience of the measurements at 1 year of age, it seemed that falling asleep was sometimes hard to accomplish, despite additional measures, like scheduling the test according to personal daily sleeping patterns of the infants and actively keeping the infants awake during the car ride to hospital. Therefore, we decided not to repeat the same measurements at 2 years of age, because with the children walking and playing by that time, falling asleep would even be harder and the risk of getting hurt by losing their balance, induced by chloral hydrate, would be increased. We chose to perform bronchial provocation tests at 2 years of age, for which no sedation is required and which reveals information about bronchial responsiveness, a relevant determinant of respiratory course in ventilated preterm infants. $174,181,195,222$ Adequate inhalation of methacholine can be achieved if their attention is sufficiently diverted.

\subsubsection{Single breath occlusion technique (SBT) to determine passive respiratory system mechanics at neonatal age and at 1 year of age}

Passive mechanical characteristics are assessed in absence of respiratory muscle activity and can be obtained during spontaneous breathing as well as during mechanical ventilation. For the occlusion techniques, besides a heated 


\section{Respiratory function in early life}

pneumotachometer to measure airflow, a valve mechanism has to be connected between endotracheal tube and ventilator tubes or to a facemask to perform occlusions at the airway opening at end-expiration. In spontaneously breathing infants, occlusions can also be performed manually at the outlet of the pneumotachometer. This technique relies on the fact that the Hering-Breuer inflation reflex is still strong in infants and young children. Consequently, if lung volume is held above FRC, either by inflation or by an occlusion at the airway opening, there is stimulation of stretch receptor activity, inhibiting subsequent inspiratory effort, resulting in prolongation of expiration with complete relaxation of respiratory muscles. ${ }^{150}$

Via a pneumotachometer and pressure transducer, signals of airflow, volume (integrated from airflow) and mouth pressure can be displayed simultaneously on a

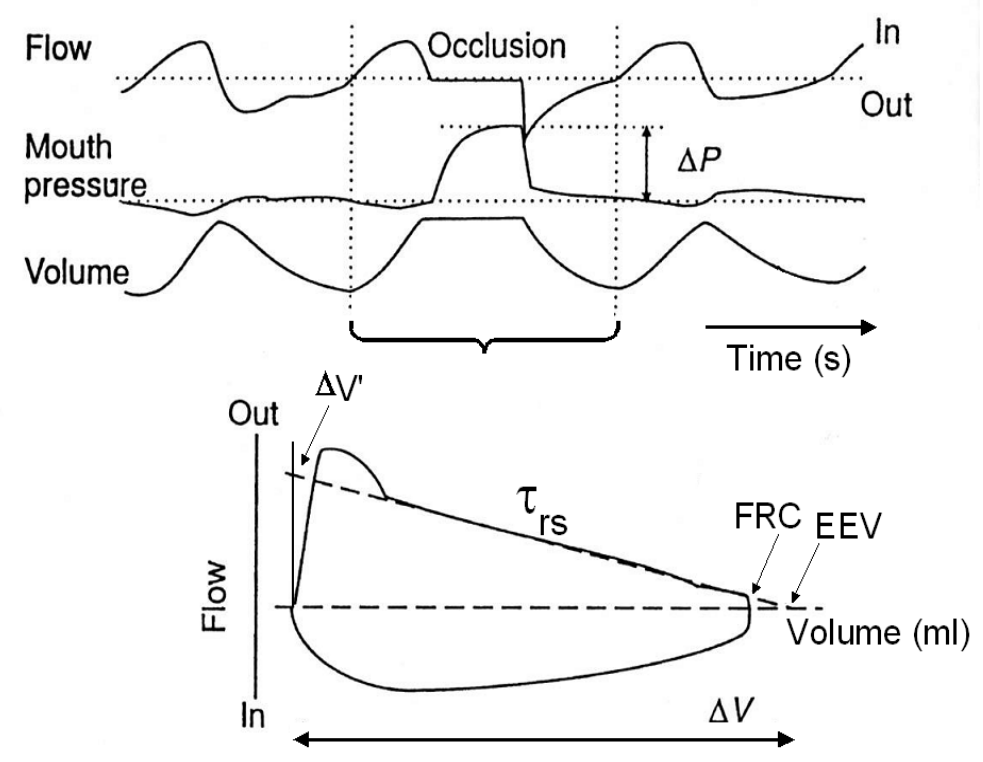

Figure 5. Single breath occlusion technique. At high inspiratory volume the airway is briefly occluded. During this occlusion no airflow is present, mouth pressure rises and reaches a plateau, and volume remains stable. The pressure difference $(\Delta P)$ between baseline and plateau is used for calculations of both $C_{\mathrm{rs}}$ and $\mathrm{R}_{\mathrm{rs}}$. After the occluded breath is rendered into a flow volume graph, $\tau_{\mathrm{rs}}$ can be determined as well as flow difference $(\Delta V)$ and volume difference $(\Delta V)$. Subsequently, $C_{\mathrm{rs}}$ can be calculated by $\Delta V / \Delta P$ and $R_{\mathrm{rs}}$ by $\Delta P / \Delta V . F R C=$ functional residual capacity; $E E V=$ elastic equilibrium volume. 
computer screen. As was described by Le Souef and colleagues in 1984, ${ }^{150}$ occlusions are performed at high tidal volumes to elicit the Hering-Breuer reflex resulting in respiratory muscle relaxation. As a consequence of the inward recoil pressure of lungs and chest wall, mouth pressure rises to reach a plateau representing equilibrium between the recoil pressures of lung and chest wall related to the amount of air present inside the lungs. The optimal duration of occlusions has still to be determined $^{163}$ and is related to respiratory rate and degree of respiratory disease affecting equilibration time. After occlusion is released, a passive expiration occurs determined by the $\tau_{\mathrm{rs}}$. The $\tau_{\mathrm{rs}}$ is reflected by the linear portion of the expiration part of the flow-volume curve (Figure 5). EEV is determined by crossing of the line representing $\tau_{\mathrm{rs}}$ and the volume-axis. The ratio between the volume of air within the lungs above EEV and the mouth pressure representing alveolar pressure during occlusion reflects $C_{\mathrm{rs}}$. By extrapolating the line representing $\tau_{\mathrm{rs}}$ towards the flow-axis the airflow during occlusion can be determined. The ratio of mouth pressure and the airflow during occlusion reflects $R_{\mathrm{rs}} .{ }^{150}$ Since $\tau_{\mathrm{rs}}$ resembles the product of $C_{\mathrm{rs}}$ and $R_{\mathrm{rs}}$, $R_{\mathrm{rs}}$ can alternatively be calculated by dividing $\tau_{\mathrm{rs}}$ by $C_{\mathrm{rs}}{ }^{180}$

If expiration occurs into the ventilator circuit, mechanical properties of this circuit are included in these calculations. Ideally, infants should expire into the atmospheric air; however, the loss of PEEP may have adverse clinical implications. There is no current consensus as to the best approach in these circumstances. ${ }^{80}$

The SBT is based on a physical model relying on several assumptions. For many respiratory function tests, the respiratory system is considered to behave like a singlecompartment model, as was already mentioned in paragraph 2.1. Consequently, both $C_{\mathrm{rs}}$ and $\mathrm{R}_{\mathrm{rs}}$ are thought to remain constant throughout expiration within the range of tidal breathing and the respiratory system should be described by a single $\tau_{\mathrm{rs}}$. Furthermore, it is assumed that the pressure at the airway opening resembles the elastic recoil pressure of the respiratory system. This can only be achieved when there is complete relaxation of respiratory muscles and complete equilibration of pressures throughout the respiratory system. Especially in neonates, brief airway occlusions do not invariably induce a respiratory pause and, in the presence of severe airway obstruction and rapid respiratory rates, complete equilibration of airway and alveolar pressures may not be reached. Finally, it is assumed that all respiratory muscles remain relaxed during the expiration following occlusion and that inertance is negligible. ${ }^{68}$

Important advantages of the SBT include the ease and non-invasive character of the test and the fact that it only takes a few minutes to carry out. Since enough time for redistribution of intrapulmonary air during a period of zero airflow is provided during airway occlusion, results are not frequency dependent. However, although respiratory muscle activity is eliminated, lung characteristics cannot be separated from chest wall characteristics. In some cases, no muscle relaxation can be achieved and thus no results obtained. During mechanical ventilation, the accuracy of the results can be substantially affected by the endotracheal tube in situ 228 and air leakage 
alongside the endotracheal tube. Also inspiratory pressures affect the level within the pressure-volume curve reflecting the degree of lung recruitment and overdistention, directly affecting $C_{\mathrm{rs}} .{ }^{132}$ Additionally, in infants with upper or lower airway obstruction there may be marked changes in resistance throughout the respiratory cycle, invalidating the assumption regarding linearity (paragraph 2.1.1.).41 Furthermore, factors causing severe deformations of the expiratory part of the flow-volume curve, like laryngeal braking, expiratory muscle activity or severe airway obstruction, may result in serious deviations of EEV. Therefore, when EEV, assessed after extrapolation of the linear part of the expiratory flow-volume curve to the volume axis, deviates by more than 10 per cent from FRC, where the expiratory flow-volume curve really crosses the volume axis at end-expiration, results should be considered unreliable. Following end-inspiratory occlusions, infants often inspire earlier than usual on the subsequent breath, such that any rise in end-expiratory resistance due to dynamic airway closure may be missed.

To ensure reliability of $C_{\mathrm{rs}}$ and $R_{\mathrm{rs}}$ assessments by SBT, occluded breaths have to meet certain criteria. There should be no evidence of leaks around the endotracheal tube or facemask. Leaks in the system or around the endotracheal tube can be easily detected as mouth pressure decreases gradually during occlusion. With gentle manual compression of the trachea present leaks can be minimized. Persisting tube leaks causing a decrease in occlusion pressure of $0.02 \mathrm{kPa}$ or more have to be excluded from further analysis. No drift of the volume signal is allowed. At least five tidal breaths should be recorded before each occlusion to allow regression to original FRC level. Passive expiration should proceed to at least 80 per cent of the volume expired during pre-occlusion tidal breathing, because early inspiration by the infant may invalidate analysis. ${ }^{68}$ Analyses were performed according to the European Respiratory Society (ERS) recommendations valid during the time of the measurements. ${ }^{61,80}$ Breath analysis was considered unreliable, when no muscle relaxation was achieved. Muscle relaxation is assumed to be present when (1) during a short occlusion period of less than 0.5 seconds mouth pressure reaches a plateau for at least 0.1 seconds during which pressure changes should be trivial (i.e. with a standard deviation (SD) of $0.01 \mathrm{kPa}$ or less), and (2) the expiration part of the flow volume curve is linear for at least 50 per cent of the volume to be expired. As a criterion for linearity, a correlation coefficient of 0.98 or more after exclusion of the final 5 per cent of the expired volume has to be reached. However, a linear expiratory flow-volume curve does not guarantee relaxation of respiratory muscles or the presence of a single passive time constant, it may also represent balanced respiratory muscle activity or reciprocal changes in compliance and resistance as lung volume decreases. We intended to use at least five occluded breaths meeting the above-mentioned criteria to calculate a mean value for both respiratory mechanics for each single measurement.

Finally, a specific description of the measurement procedure and equipment that was used during neonatal measurements and measurements at 1 year of age is 


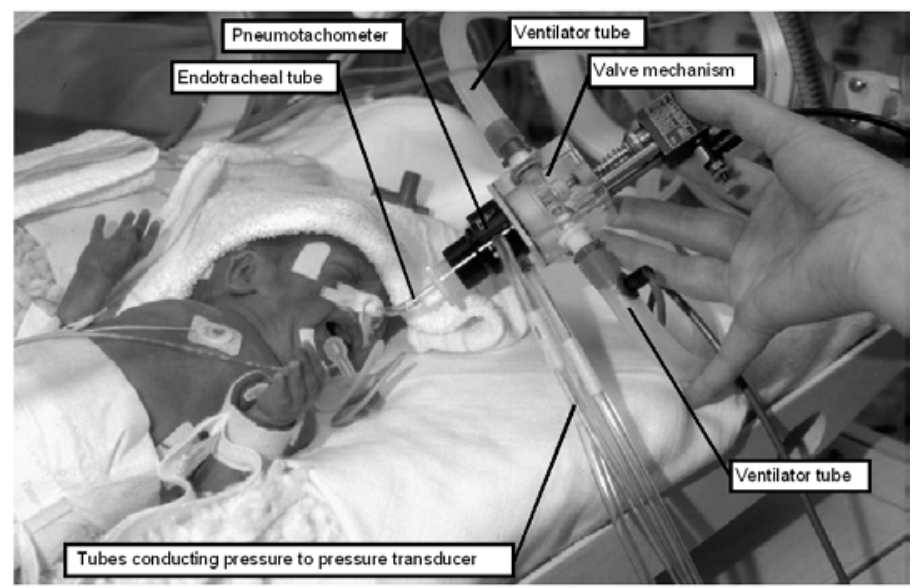

Figure 6. Respiratory function testing during the neonatal period at the neonatal intensive care unit.

provided. During the neonatal period mechanically ventilated preterm infants were measured using a heated Lilly-type pneumotachometer (8410A, linear range 0-10 $\mathrm{L} / \mathrm{min}$, dead space $1.3 \mathrm{ml}$, resistance $0.96 \mathrm{kPa} / \mathrm{L} \cdot \mathrm{s}$ at $5 \mathrm{~L} / \mathrm{min}$; Hans Rudolph Inc., Kansas City, MO, USA) placed between endotracheal tube and ventilator tubes (Figure 6). A pressure transducer (Honeywell, type 163PC01D75, Morristown, NJ, USA) and an IBM compatible personal computer with a Pentium processor running the software package Respiratory Analysis System Program (Version 007.17, Physio Logic, High Clere, Great Britain) were used to record signals at a sampling rate of 125 $\mathrm{Hz}$ using a 12 bit analogue-to-digital converter (LabMaster DMA, Scientific Solutions, Mentor, OH, USA). Mouth pressure and flow signals were obtained after calibration before each single measurement. For flow calibration, a calibrated rotameter (Air 0-15 $\mathrm{L} / \mathrm{min}$, Medec, Aalst, Belgium) was used followed by a volume calibration using a precision syringe of $50-\mathrm{ml}$ containing the actual gas mixture. For pressure calibration, a pressure transducer tester (Veri-Cal 650-900, Utah Medical Products Inc., Midvale, UT, USA) was used.

At 1 year of age, the SBT was performed using a heated Lilly-type pneumotachometer $(3500 \mathrm{~B}$, linear range $0-35 \mathrm{~L} / \mathrm{min}$, dead space $8.74 \mathrm{ml}$, resistance 0.32 $\mathrm{kPa} / \mathrm{L} \cdot \mathrm{s}$ at $17.5 \mathrm{~L} / \mathrm{min}$; Hans Rudolph Inc., Kansas City, MO), attached to a facemask (Laerdal Infant Mask No.1, Laerdal BeNelux BV, Valkenswaard, The Netherlands; dead space $10 \mathrm{ml}$ ) covered with therapeutic putty (Magic Putty, medium, Oldelft Benelux BV, Delft, The Netherlands) to prevent air leakage (Figure 7). Infants were 


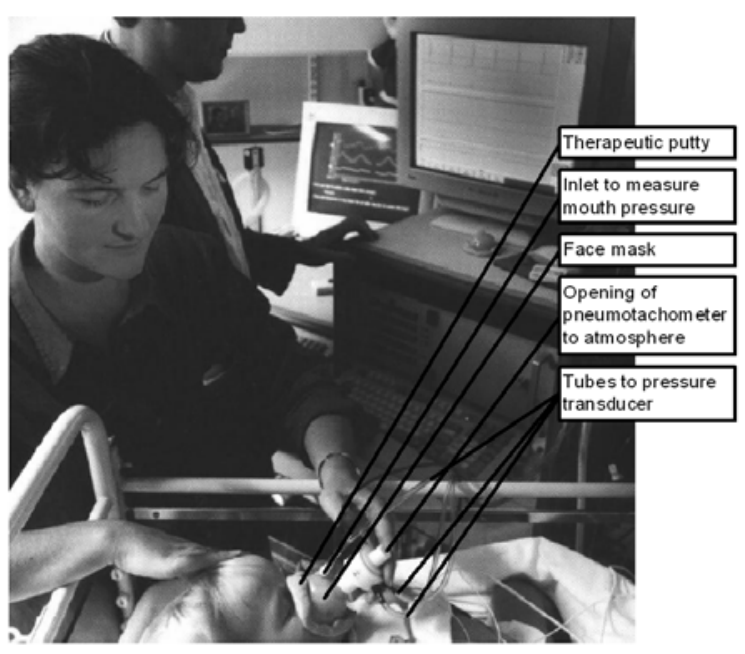

Figure 7. Respiratory function testing at 1 year of age. Infants were sedated while breathing through a facemask and pneumotachometer. Occlusions were performed manually at the opening of the pneumotachometer to atmosphere. For FRC measurements the pneumotachometer was removed and the valve attached to the spirometer closed system was connected to the facemask.

sedated with $50-100 \mathrm{mg} / \mathrm{kg}$ chloral hydrate and measurements were performed in supine position with the head end elevated at approximately 30 degrees relative to horizontal. Calibration procedure was performed with a precision syringe containing $100 \mathrm{ml}$ of air.

\subsubsection{Methacholine provocation test at 2 years of age}

Provocation tests were initially applied in older children and adults, since conventional methods included peak expiratory flow assessments (PEF) ${ }^{29}$ or spirometry measures (forced expiratory volume in 1 second) ${ }^{140}$ to determine the response to non-specific irritants. The introduction of other measures, like $V_{\max , \mathrm{FRC}}{ }^{181} \mathrm{R}_{\mathrm{aw}}$, $G_{\text {aw }}{ }^{140}$ and chest auscultation with or without the assessment of $\mathrm{SaO}_{2}, 9,225$ made this technique also feasible in infants and young children. The simplest method to perform in infants and young children is probably the method described by Avital and colleagues in 1988. ${ }^{9}$ For this technique, chest auscultation is used to determine the threshold value of methacholine. Compared to the conventional method, chest auscultation seems to correlate well with spirometer measures in older children. ${ }^{9}, 224,225$ 
Chest auscultation is performed before the periods of nebulizing are started. Continuous $\mathrm{SpO}_{2}$ monitoring takes place by attaching a pulse oximeter to a toe of the child. Subsequently, nebulizing is started for periods of 2 minutes starting with isotonic saline followed by increasing concentrations of methacholine. ${ }^{34}$ After each nebulizing period, chest auscultation is performed. If wheezing occurs during chest auscultation or oxygen saturation falls below 90 per cent the preceding methacholine concentration is recorded as the threshold value. Finally, salbutamol is administered to relieve bronchial constriction.

According to the literature, the method described by Avital and colleagues seems to be safe, effective, easily applicable, and clinically significant in young children. $918,98,225$ However, especially in young children, the method is time consuming and without sedation only applicable from 2 years of age. At this age, great effort is required to divert the attention of the child.

Besides respiratory disease, airway responsiveness is affected by pulmonary hyperinflation and age, and these factors should therefore be taken into consideration when results are interpreted. Since the nebulizing techniques that are used nowadays are similar at different ages, the concentration of inhaled methacholine may relatively increase with decreasing ages, because dilution of the nebulizing solution of methacholine by environmental air is less in young children compared to older children and adults, in whom inspiratory airflow during tidal breathing will exceed the airflow used to nebulize methacholine $(6 \mathrm{~L} / \mathrm{min})$. Hence, the increased bronchial responsiveness at younger ages may exist due partly to exposure to relatively increased methacholine concentrations. ${ }^{148}$ Lack of standardization of the method in young children limits clinical application. However, in studies of children of the same age, results are less determined by interindividual differences of methacholine exposure. Still, factors like excessive commotion and crying during the test may induce additional bronchial constriction. There is also a large deficit of studies investigating bronchial responsiveness in healthy children. In conclusion, the method needs further validation before implementation in clinical practice should be encouraged in such young children.

A specific description of the measurement procedure and equipment that was used during measurements at 2 years of age is provided. Bronchial challenge tests were carried out using a continuous inhalation tidal breathing method as described by Cockroft and colleagues. ${ }^{34}$ Before the test was started, chest auscultation was performed. Isotonic saline was administered for 2 minutes from a nebulizer (Up-draft II nebuliser, Hudson Respiratory Care Inc., Temecula, CA), connected to a flow meter (Endomed Air $20^{\circ} \mathrm{C}$ 0-17 L/min, Endomed B.V., Didam, The Netherlands) delivering a flow rate of $6 \mathrm{~L} / \mathrm{min}$ out of the hospital circuit of compressed air (Figure 8). Chest auscultation was repeated shortly after saline nebulization. If no wheeze was heard, a serial inhalation of doubling concentrations of methacholine was started from a concentration of $0.15 \mathrm{mg} / \mathrm{ml}$ every 5 minutes to a maximum concentration of 9.6 


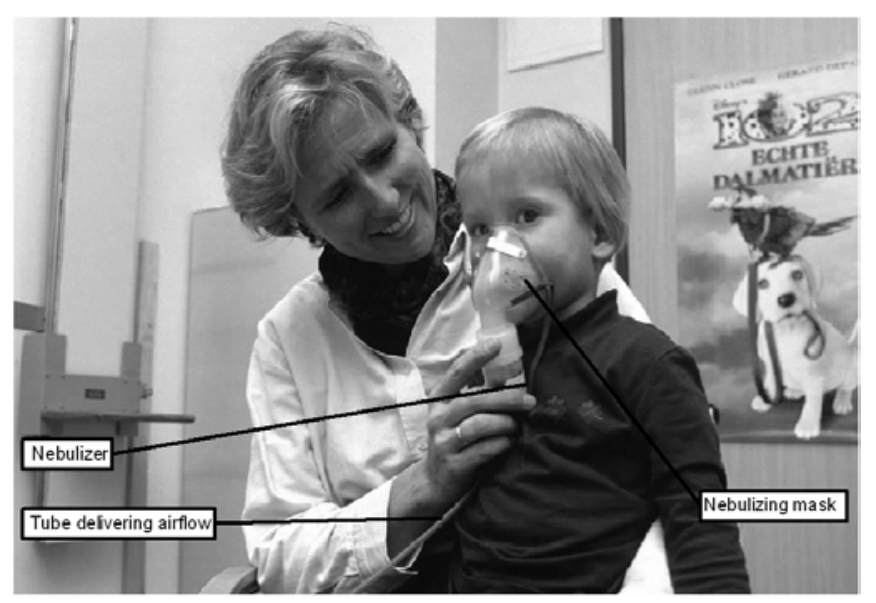

Figure 8. Respiratory function testing at 2 years of age. A nebulising mask is held loosely in front of the child's face producing nebulized methacholine for periods of 2 minutes.

$\mathrm{mg} / \mathrm{ml}$ (i.e. $0.15,0.30,0.60,1.20,2.40,4.80$ and $9.60 \mathrm{mg} / \mathrm{ml}$ consecutively). Each methacholine concentration was nebulized for 2 minutes. Shortly after each inhalation, chest auscultation was performed by the same observer every time.

The response to the challenge was monitored by a pulse oximeter (Nellcor $\mathrm{N}$ 395, Mallinckrodt Europe B.V., 's-Hertogenbosch, The Netherlands) attached around a toe. Baseline values of $\mathrm{SpO}_{2}$ were attained after a short period of stabilization. The challenge was considered positive and terminated if an audible wheeze was present over the chest, if $\mathrm{SpO}_{2}$ fell below 90 per cent (for at least ten seconds) or if the final methacholine concentration of $9.6 \mathrm{mg} / \mathrm{ml}$ was reached, whatever occurred first. At that moment, the provocative concentration of methacholine (PC) was recorded, as was the $\mathrm{SpO}_{2}$. Children inhaled $800 \mu \mathrm{g}$ of Salbutamol via a Babyhaler to decrease bronchusconstriction. When children were wheezing before initiation of the test, their parents were kindly requested to come back for the test a few days or weeks later.

\subsubsection{Pulse oximetry for monitoring during provocation tests at 2 years of age}

At 2 years of age, $\mathrm{SpO}_{2}$ was monitored during methacholine provocation tests to check for desaturations that may follow methacholine provocation. Additionally, $\mathrm{SpO}_{2}$ was used to determine the threshold value of methacholine when no wheeze was heard by auscultation and $\mathrm{SpO}_{2}$ decreased below 90 per cent. In general, appropriate flexible sensors should be attached around a toe or finger and, if $\mathrm{SpO}_{2}$ values are low 
together with a low heart rate, the sensor should be repositioned until a reliable signal is obtained.

Although this technique was developed in 1974,7 it took another 10 years before it was introduced in clinical practice. The technique is based on the difference in absorption spectra of oxygenated and deoxygenated haemoglobin. Oxygenated haemoglobin absorbs more light in the infrared band $(850-1000 \mathrm{~nm})$ and deoxygenated haemoglobin absorbs more light in the red band $(600-750 \mathrm{~nm})$. The oximeter probe emits light at two specific wavelengths (at 660 and $940 \mathrm{~nm}$ ), being transmitted through tissue (e.g. a finger or toe) and measured by a photodetector. The ratio of the light absorbances at the two wavelengths correlates with the proportion of oxygenated to deoxygenated haemoglobin in the tissue. To select only the pulsating part of the tissue, which correlates with arterial $\mathrm{O}_{2}$ saturation and is characterized by changes in the total light permitted (Figure 9), the pulse oximeter divides the absorbances at the peaks by those at the corresponding troughs, thereby obtaining a 'pulse-added' absorbance that is independent of the absorbance characteristics of the non-pulsating parts of the tissue. A major advantage of the technique is the ease of usage, because no calibration or heating of the skin is required to immediately provide information about arterial oxygenation. Response time is faster compared to $\mathrm{P}_{\mathrm{tc}} \mathrm{O}_{2}$ monitoring, but may vary depending on the averaging time of the pulse oximeter. Sensitivity and specificity of detecting hypoxemia and hyperoxemia differs substantially between different brands, but is most of the time better than $\mathrm{P}_{\mathrm{tc}} \mathrm{O}_{2}$ monitoring. Most manufacturers claim that 95 per cent of the measured values will be within 4-6 per cent of the actual $\mathrm{SaO}_{2}$. Pulse oximetry depends less on peripheral perfusion. However, improper placement and sensor type may affect the precision of a pulse oximeter by optical shunting of ambient light. Furthermore, skin burns have been described in situations where one brand of sensor was used with a different brand of instrument, the sensor surface was damaged, but also without any apparent cause. ${ }^{200}$ Since the pulsating signal is only $1-5$ per cent of the total absorbance

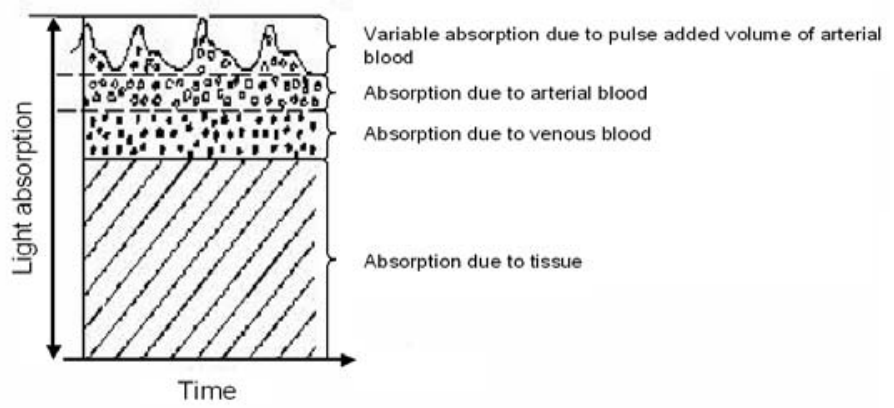

Figure 9. Basal and pulsatile components in the light absorbances measured by the pulse oximeter. 


\section{Respiratory function in early life}

measured, the instrument is very sensitive to sudden changes in background signal due to, for example, body movements. To minimize this, the light plethysmographic waveforms should be judged simultaneously to see whether they are distorted. Abnormal haemoglobins or dyes with abnormal absorption spectra may also affect the accuracy of the pulse oximeter. Since pulse oximeters rely on algorithms based on in vitro measurements of $\mathrm{SaO}_{2}$ obtained from healthy adult volunteers within a range of 70 to 100 per cent, for saturations below this range algorithms are simply extrapolated. This procedure may lead to an underestimation of true degree of hypoxemia and has led to recommendations that in these situations $\mathrm{SaO}_{2}$ should be determined in order to measure the true severity of hypoxemia. ${ }^{200}$

For $\mathrm{SpO}_{2}$ monitoring during the methacholine provocation tests at 2 years of age, a Nellcor pulse oximeter was used (Nellcor N-200 pulse oximeter, Mallinckrodt Europe BV, 's-Hertogenbosch, The Netherlands) with an appropriate sensor (Nellcor I-20 oxisensor 2, Mallinckrodt Europe BV, 's-Hertogenbosch, The Netherlands). The sensor was attached around a toe and, when reliable $\mathrm{SpO}_{2}$ signals were being displayed, the child's sock was put back on. When at the provocation test $\mathrm{SpO}_{2}$ values decreased below 90 per cent, the test was ended. 


\title{
CHAPTER 3
}

\section{Correction factors for oxygen and flow-rate effects on neonatal Fleisch and Lilly pneumotachometers}

\author{
Yvonne Snepvangers \\ Peter de Winter \\ Huibert Burger \\ Hens Brouwers \\ Jan Bogaard \\ Kors van der Ent
}

Published in: Pediatric Critical Care Medicine, 2003;4:227-232 



\subsection{Abstract}

Objective: To assess the effects of different oxygen concentrations and flow rates on the measurement errors of neonatal pneumotachometers in heated and unheated situations, and to develop correction factors to correct for these effects.

Design: Prospective laboratory study.

Setting: Outpatient clinic with equipment in a standardized setting.

Subjects: Neonatal pneumotachometers.

Interventions: In standardized conditions the tested pneumotachometer was calibrated at a flow rate of $3 \mathrm{~L} / \mathrm{min}$ with 60 per cent oxygen and was set in series with a closed spirometer system being used as a reference. Different airflow levels (1-9 $\mathrm{L} / \mathrm{min}$ ) and oxygen concentrations (21-100\%) were infused into the closed system with pneumotachometer and spirometer.

Measurements and Main Results: The pneumotachometers were significantly affected by changing oxygen concentrations $(p<0.01)$ and increasing flow rates $(p<0.01)$ increasing the actually measured flow rate. Correction factors, developed by multiple regression analysis, significantly reduced the overall maximum errors of the pneumotachometers from $-1.1-0.6 \mathrm{~L} / \mathrm{min}$ to $-0.5-0.4 \mathrm{~L} / \mathrm{min}$.

Conclusions: The effects of changes in oxygen concentrations and flow rates on neonatal pneumotachometers could be considerably decreased by the use of correction factors as were calculated in this study. This will preclude frequent calibration procedures with actual flow and oxygen levels during changes in experimental settings.

\subsection{Introduction}

In research as well as in clinical practice, accurate measurements of respiratory gas flow rates are important. Pneumotachometers are used in set-ups for estimating lung volumes, respiratory system compliance and resistance. Pneumotachometers can be based on several principles, like linear resistive pneumotachometers, turbine flowmeters, thermal devices, ultrasonic flowmeters, polyvinylidene fluoride piezoelectric film flowmeters, non-linear differential pressure-based transducers, linear differential pressure devices and flow plethysmography. ${ }^{68}$ Since the dead space of pneumotachometers has been sufficiently reduced they can be used in neonates. There are, however, more specific conditions to be taken into account during lung function testing in neonates, especially when they are mechanically ventilated. According to Hagen-Poiseuille's law, changes in physical characteristics of a respiratory gas (composition, temperature, relative humidity, and pressure) ${ }^{21}$ directly affect pneumotachometer flow measurements. 47, 69, 73, 92, 96, 97, 110, 212, 248, 250, 263, 269 


$$
\mathrm{p} D=(8 \cdot L \cdot \eta \cdot \nu) /(\pi \cdot r 4)
$$

Where $\mathrm{p} D$ is the decrease in pressure; $L$ the length of tube over which pressure decrease is measured; $\eta$ the viscosity of gas; $v$ the velocity; and $r$ the radius of tube over which pressure decrease is measured

In addition, turbulence, ${ }^{279}$ the geometry of the tubes connecting the pneumotachometers ${ }^{142}$ and the dimensions of the resistive element ${ }^{279}$ influence flow recordings by pneumotachometers. In experimental settings at a neonatal intensive care unit (NICU) water can accumulate on the resistive element of pneumotachometers increasing resistance and causing turbulence, leading to measurement errors. Heating of the pneumotachometer diminishes this effect. In the NICU flow rates and oxygen concentrations are often changed leading to a varied accuracy of flow signals. In recently developed neonatal ventilators algorithms are incorporated to reduce the measurement error of their pneumotachometers. However, most of them (Stephanie and Dräger among other companies) only include corrections for body temperature and pressure saturated with $\mathrm{H}_{2} \mathrm{O}$ vapour (BTPS). The Viasys Avea and the VIP Bird neonatal and paediatric ventilators additionally correct for altitude (barometric pressure). However, to our best knowledge, no correction is made for actual oxygen concentration and flow rate. We investigated the accuracy of the two most frequently used neonatal pneumotachometers ${ }^{17}$ to measure lung function in experimental settings. Both are based on the linear resistive principle: a Fleisch type pneumotachometer (consisting of parallel capillary tubes) ${ }^{92}$ and a Lilly type pneumotachometer (containing a fine wire mesh screen). ${ }^{212}$ We studied measurement errors secondary to changing measurement conditions (e.g. flow rate, oxygen concentration and heating of the devices). Correction factors were calculated in order to prevent the need for frequent calibration procedures at actual flow rates and oxygen concentrations.

\subsection{Material and methods}

A Lilly pneumotachometer (8410A, Hans Rudolph Inc., Kansas City, MO; linear range $0-10 \mathrm{~L} / \mathrm{min}$ ) and a Fleisch pneumotachometer (No. 00, General Medical Corp., Erie, PA; linear range 0-9 L/min) were tested. Pressure differences across the resistive element of both pneumotachometers were measured with a differential pressure transducer (type 163PC01D75, Honeywell, Morristown, NJ). Air and oxygen from the hospital circuit were mixed with an oxygen-air mixer (961 Siemens-Elema, Siemens, The Hague, The Netherlands) and flow rates were roughly set by a rotameter (Air 0 $15 \mathrm{~L} / \mathrm{min}$, Medec, Aalst, Belgium) (Figure 1). 


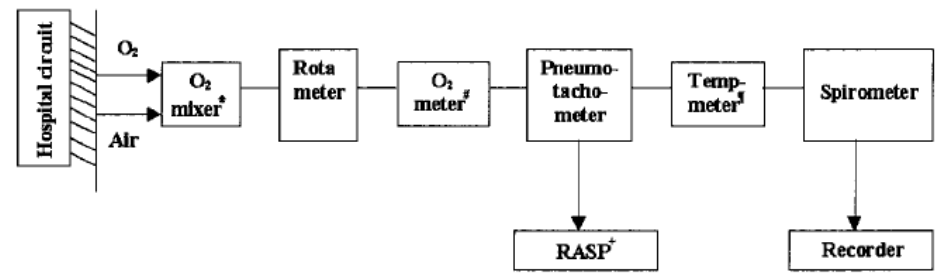

Figure 1. Schematic view of the experimental setting during the measurements. *oxygen-air mixer; \# Oxygen concentration meter; Ithermometer; ${ }^{+}$RASP, Respiratory Analysis System Program.

The oxygen concentration (at $30 \mathrm{~cm}$ before the pneumotachometer) as well as the temperature (at $80 \mathrm{~cm}$ after the pneumotachometer) inside the system were recorded by an oxygen meter (Teledyne TED 60T, Teledyne Brown Engineering, Huntsville, Alabama) and a thermometer (Fluke $51 \mathrm{~K} / \mathrm{J}$ thermometer, Fluke Nederland B.V., Eindhoven, The Netherlands), respectively. A 10-litre-rolling-seal spirometer (Masterscreen-FRC Jaeger complete system, Erich Jaeger GmbH, Hoechberg, Germany) was used as a gold standard for volume against time. Connections between different parts of the equipment were formed by ribbed rubber tubes with an inner diameter of $2 \mathrm{~cm}$ and an outer diameter of $2.9 \mathrm{~cm}$ before and after the pneumotachometer, and the standard ribbed tubes belonging to the spirometer with an inner diameter of $3.0 \mathrm{~cm}$ and an outer diameter of $3.2 \mathrm{~cm}$ connected the former tubes from the point of temperature assessment to the spirometer. Flow signals from the pneumotachometers were processed by computer using the software package RASP (Respiratory Analysis System Program, Physio Logic, High Clere, Great Britain).

We calibrated the pneumotachometer system at an oxygen concentration of 60 per cent to keep the maximum error, expected to be caused by the most extreme oxygen concentrations, as small as possible. Flow calibration was performed at zero flow and at a maximum of $3 \mathrm{~L} / \mathrm{min}$ according to the spirometer. Since flow rates measured by the spirometer can only be determined afterwards all measured values were multiplied with a new calibration factor determined afterwards at $3 \mathrm{~L} / \mathrm{min}$ and 60 per cent oxygen. This calibration procedure was performed for each measurement session separately.

At the start of the measurements an oxygen concentration of 21 per cent and a steady flow rate of about $1 \mathrm{~L} / \mathrm{min}$ (according to the spirometer) was used. We repeated the same procedure at an oxygen concentration of 21 per cent and a steady flow rate of $2 \mathrm{~L} / \mathrm{min}$, followed by $3,4,6$ and $9 \mathrm{~L} / \mathrm{min}$. Afterwards we tested the other oxygen concentrations of $30,40,50,60,70,80,90$ and 100 per cent at the same steady 
flow rates. We repeated half the session, i.e. the same measurement procedures in the opposite order at 100, 80, 60, 40 and 21 per cent oxygen every time at 1, 3, 6 and 9 $\mathrm{L} / \mathrm{min}$.

The complete procedure was performed for each pneumotachometer, i.e. the Lilly and the Fleisch pneumotachometer heated as well as unheated. Recordings during every measurement were continued until the spirometer with a volume of $10 \mathrm{~L}$. was completely filled, except at a flow rate of $1 \mathrm{~L} / \mathrm{min}$ a maximum total volume of 6 L. mean was achieved. No lower volumes were accepted to prevent measurement errors due to a potential inaccuracy of the spirometer. Before and after each measurement procedure calibration settings were checked with a precision syringe of $50 \mathrm{ml}$ independently from the spirometer.

When this check revealed an error of more than 2.5 per cent the whole measurement procedure was repeated. Paired-samples $t$-tests were performed to estimate significant differences of the measurement errors between calibration level and the lowest and highest oxygen concentrations and flow rates. Bonferroni correction for multiple comparisons was performed.

Multiple regression analyses were used to derive correction equations for each pneumotachometer. We used a stepwise forward regression technique with the reference flow (i.e. the flow recorded by the spirometer) as the dependent variable. The flow measured by the pneumotachometer, its product term with the oxygen concentration and its square were included as independent variables. Variables were added on the basis of statistical significance $(p<0.05)$ or when they substantially improved the fit of the model, according to the distribution of the residuals left. To enable comparison between pneumotachometers data were presented as relative measurement errors calculated from the regression equations. This means that the differences between the flow rates measured by the pneumotachometer and the calculated spirometer flow rates (dependent variable in regression equation) after entering the pneumotachometer flows in the equations were presented. These differences were divided by the spirometer values and expressed as percentages. Since variances explained by the regression equations were very high $\left(R_{2}>0.99\right)$ no important deviation from the real measured data was to be expected. To test the improvement due to the use of the correction factors following from the regression equations Bland\&Altman plots ${ }^{20}$ were used in which the differences between spirometer and pneumotachometer were plotted against the mean of their flow rates. The paired samples $t$-test was again used to estimate any significant improvements due to the use of these correction factors.

Polynomials of different pneumotachometer flow errors relative to spirometer flow were calculated with the multiple regression equations using arbitrary oxygen concentrations and flow rates. The Statistical Product and Service Solutions 10.0 (SPSS Inc, Chicago, IL, USA) for Windows was used for the statistical calculations. 


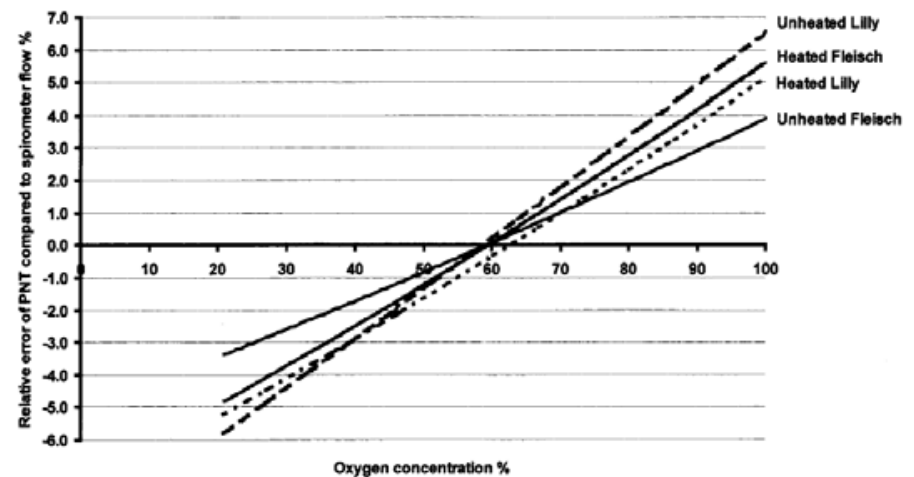

Figure 2. Trends of relative measurement errors of the pneumotachometers (PNT) at the calibrated flow rate $(3 \mathrm{~L} / \mathrm{min})$ at different oxygen concentrations.

\subsection{Results}

The temperature measured inside the closed circuit at $80 \mathrm{~cm}$ after the pneumotachometer did not change by more than $1.3^{\circ} \mathrm{C}$ (ranges for each experiment, 21.9-23.2; 20.2-21.1; 21.1-21.7; 20.3-21.4) for the unheated pneumotachometers and not more than $2.4^{\circ} \mathrm{C}$ (ranges for each experiment, 20.7-23.1; 20.6-21.7; 22.1-23.1; 21.3-23.1) for the heated pneumotachometers within each experiment. Changes of barometric pressure and humidity during the measurement procedures were not present or very small reaching maximum values of $0.2 \mathrm{kPa}$ and 4 per cent, respectively.

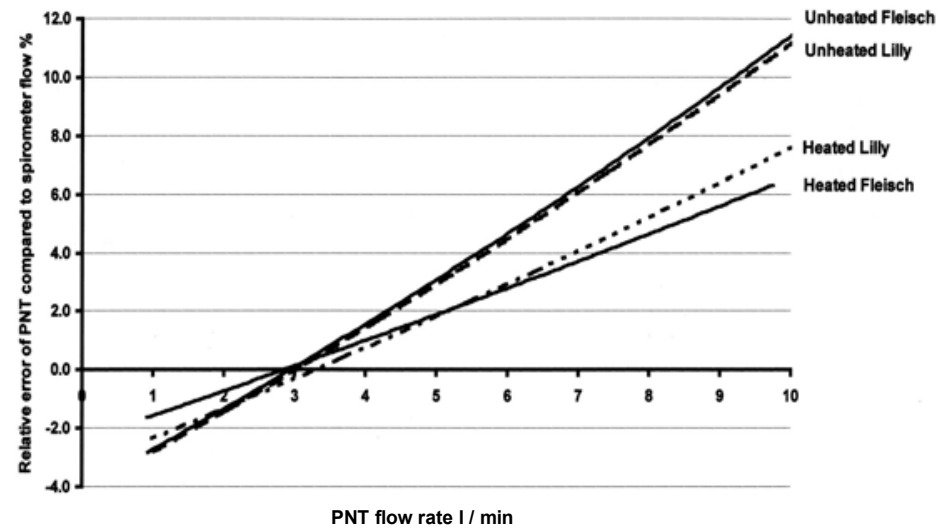

Figure 3. Trends of relative measurement errors of the pneumotachometers (PNT) at the calibrated oxygen concentration $(60 \%)$ at different flow rates. 
Oxygen and flow-rate effects on pneumotachometers

Table 1. Multiple regression equations for each separate pneumotachometer.

\begin{tabular}{|c|c|c|}
\hline Pneumotachometer & Regression Equation & $r^{2}$ \\
\hline Heated Fleisch & Flow $^{a}=1.10 \cdot$ Flow $^{\mathrm{b}}-0.0013 \cdot$ Flow $_{M} \cdot \mathrm{O}_{2} \%-0.086 \cdot\left(\text { Flow }_{M}\right)^{2}$ & $>.99$ \\
\hline Unheated Fleisch & Flow $_{\mathrm{R}}=1.10 \cdot$ Flow $_{M}-0.0009 \cdot$ Flow $_{M} \cdot \mathrm{O}_{2} \%-0.015 \cdot\left(\text { Flow }_{M}\right)^{2}$ & $>.99$ \\
\hline Heated Lilly & Flow $_{\mathrm{R}}=1.11 \cdot$ Flow $_{\mathrm{M}}-0.0013 \cdot$ Flow $_{\mathrm{M}} \cdot \mathrm{O}_{2} \%-0.011 \cdot\left(\text { Flow }_{\mathrm{M}}\right)^{2}$ & $>.99$ \\
\hline Unheated Lilly & Flow $_{R}=1.14 \cdot$ Flow $_{M}-0.0013 \cdot$ Flow $_{M} \cdot \mathrm{O}_{2} \%-0.014 \cdot\left(\text { Flow }_{M}\right)^{2}$ & $>.99$ \\
\hline
\end{tabular}

Considerable effects of oxygen concentration, shown in Figure 2, were underlined by the relative error being significantly different at 21 and 100 per cent oxygen (at $3 \mathrm{~L} / \mathrm{min}$ ) compared to calibration level $(60$ per cent oxygen at $3 \mathrm{~L} / \mathrm{min}$ ) in all pneumotachometers $(p<0.02)$. All pneumotachometers showed a comparable increase of the relative measurement error at rising oxygen concentrations, the most prominent for the unheated Lilly pneumotachometer. However, differences between pneumotachometers were small. Compared to calibration level $(3 \mathrm{~L} / \mathrm{min}$ at 60 per cent oxygen) the measurement errors were significantly different at $1 \mathrm{~L} / \mathrm{min}$ and 9 $\mathrm{L} / \mathrm{min}$ at 60 per cent oxygen in all pneumotachometers $(p<0.02)$ (Figure 3). An increasing measurement error became apparent in all pneumotachometers above calibration flow rate. The relative measurement error also increased at lower flow rates. Again differences between pneumotachometers were relatively small.

Multiple regression analyses yielded correction equations for each pneumotachometer (Table 1). With these regression equations a corrected flow could be calculated from the measured flow at different levels of oxygen concentration and

Table 2. Mean differences, limits of agreement and maximum error ranges in $\mathrm{L} / \mathrm{min}$ according to Bland \& Altman analysis ${ }^{20}$ of the differences between the pneumotachometers and the spirometer.

\begin{tabular}{|c|c|c|c|c|c|}
\hline \multirow[t]{2}{*}{ Pneumotachometer } & \multirow{2}{*}{$\begin{array}{l}\text { Mean } \\
\text { difference }\end{array}$} & \multirow[t]{2}{*}{ Limits of Agreement } & \multicolumn{3}{|c|}{ Maximum Error Range } \\
\hline & & & $\min$ & $\max$ & (difference) \\
\hline \multicolumn{6}{|l|}{ Before correction } \\
\hline Heated Fleisch & -0.18 & $-0.83,0.46$ & -1.09 & 0.40 & (1.5) \\
\hline Unheated Fleisch & -0.20 & $-1.02,0.62$ & -1.26 & 0.51 & (1.8) \\
\hline Heated Lilly & -0.36 & $-1.33,0.61$ & -1.08 & 0.17 & (1.3) \\
\hline Unheated Lilly & -0.13 & $-1.01,0.74$ & -1.28 & 0.58 & (1.9) \\
\hline \multicolumn{6}{|l|}{ After correction } \\
\hline Heated Fleisch & -0.01 & $-0.26,0.24$ & -0.28 & 0.35 & $(0.6)$ \\
\hline Unheated Fleisch & -0.01 & $-0.34,0.31$ & -0.29 & 0.39 & $(0.7)$ \\
\hline Heated Lilly & -0.04 & $-0.32,0.24$ & -0.51 & 0.37 & $(0.9)$ \\
\hline Unheated Lilly & -0.02 & $-0.28,0.24$ & -0.46 & 0.37 & $(0.8)$ \\
\hline
\end{tabular}



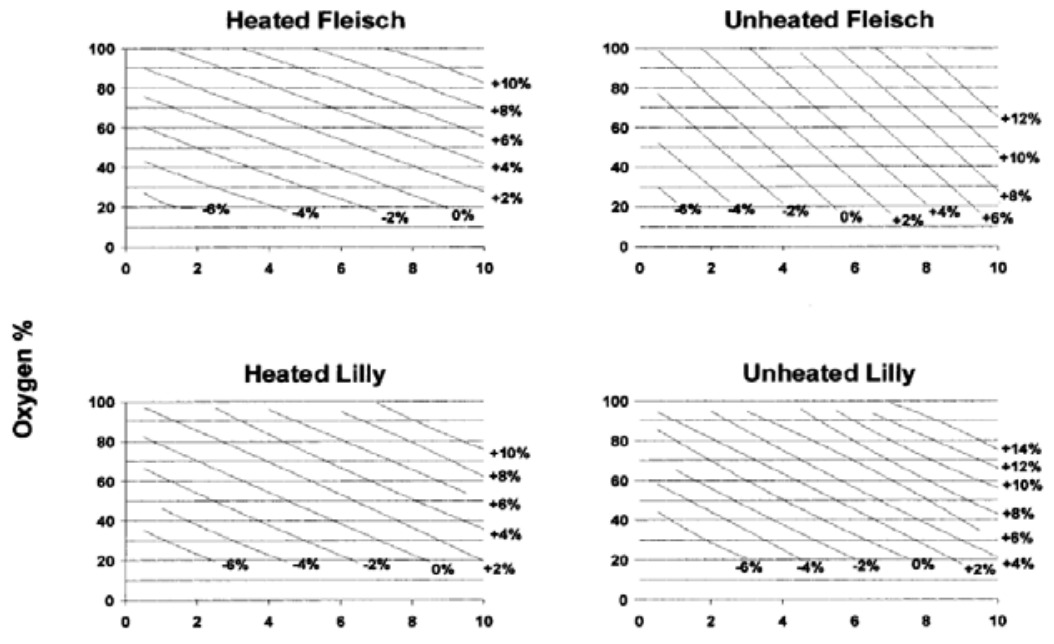

Flow rate L/min

Figure 4. Relative measurement errors of the pneumotachometers relative to the reference flow rate of the spirometer plotted as polynomials according to oxygen concentration and flow rate. Underestimation of flow rates by the pneumotachometer is indicated as a negative relative error and overestimation of flow rates as a positive relative error.

flow rate. As illustrated in Table 2, correction of the measured values with pneumotachometers resulted in a significant decrease of the measurement errors $(p<0.03)$ in all pneumotachometers. Before correction a mean measurement error varied between -0.13 and $-0.36 \mathrm{~L} / \mathrm{min}$ depending on the pneumotachometer tested. After correction this was significantly reduced to -0.01 and $-0.04 \mathrm{~L} / \mathrm{min}$. Limits of agreement, corresponding with two standard deviations of the differences between pneumotachometer and spirometer, even exceeded $1 \mathrm{~L} / \mathrm{min}$ in some cases, while these limits remained below $0.40 \mathrm{~L} / \mathrm{min}$ after correction. Maximal error ranges showed a comparable pattern. To avoid the use of these complex correction equations in practice we calculated polynomials to estimate the relative measurement errors at arbitrary oxygen concentrations and flow rates (Figure 4).

The heated Fleisch pneumotachometer showed the most dispersed polynomials in horizontal direction indicating that the relative measurement error did not increase as fast at rising flow levels as it did in the heated and unheated Lilly pneumotachometers and the unheated Fleisch pneumotachometer. The heated Fleisch curve in Figure 3 also seemed to be the flattest. Judging the dispersion of the curves in Figure 4 in vertical direction the increase of the measurement errors based upon a rise 
in oxygen concentration was most obvious in the unheated Lilly pneumotachometer and least obvious in the unheated Fleisch pneumotachometer. This was also demonstrated in Figure 2.

\subsection{Discussion}

Both the Fleisch and Lilly pneumotachometers were significantly affected by increasing oxygen concentrations from 21 to 100 per cent and increasing flow rates from 1 to $9 \mathrm{~L} / \mathrm{min}$ increasing the actually measured flow rate in the heated as well as in the unheated situation. Multiple regression analysis yielded correction factors to correct for these effects resulting in a significantly improved accuracy of the flow measurements in almost all pneumotachometers. This means that after a calibration at 0 and $3 \mathrm{~L} / \mathrm{min}$ and 60 per cent oxygen and correction for the influences of oxygen and flow rate reliable flow measurements can be achieved in the appropriate flow range and at all oxygen concentrations with these neonatal pneumotachometers.

Although linear resistance pneumotachometers are supposed to be used heated to avoid water accumulation, we tested them unheated as well. In practice sometimes unheated probes are used. Therefore, it would be interesting to correct for measurement errors caused by differences in heating assuming that no detectable water accumulation had occurred in the unheated situation. Our results confirmed that when heated pneumotachometers were used the error caused by flow rates, but not by oxygen concentrations, was less compared to when they were tested unheated. However, the actual gas temperature measured behind the heated pneumotachometer was also flow dependent. At lower flow rates the gas had more time to warm up compared to higher flow rates. Hence, the temperature difference that we measured between low $(1 \mathrm{~L} / \mathrm{min})$ and high $(9 \mathrm{~L} / \mathrm{min})$ flow rates was $10^{\circ} \mathrm{C}$ for the heated Fleisch pneumotachometer $\left(40.0\right.$ to $29.9^{\circ} \mathrm{C}$ ). For the heated Lilly pneumotachometer the maximum temperature difference was $5^{\circ} \mathrm{C}\left(30-24.9^{\circ} \mathrm{C}\right)$. Maximal temperature differences of $10^{\circ} \mathrm{C}$ and $5^{\circ} \mathrm{C}$ cause maximal viscosity changes of 2.6 per cent and 1.3 per cent, respectively. ${ }^{17}$ Temperature decreased at higher flow rates. So, levelling temperatures at all flows implies that the temperature at high flow rates should increase or the temperature at low flows should decrease. Increasing the temperature at high flows will increase gas viscosity and, thereby, the measurement error. The other possibility, decreasing temperature at low flow rates, results in the negative flow error to be increased. In both cases the slope of the lines of both heated pneumotachometers will rise approximating the ones of the unheated pneumotachometers.

Environmental changes during our experiments, like changes in temperature, barometric pressure and humidity outside the closed circuit, were negligible because changes were minimal and because calibration procedures were performed before 
each measurement procedure. The maximum changes in humidity would have caused a decrease in viscosity of 0.1 per cent. Barometric pressure changes would only give a measurable effect on viscosity when exceeding $500 \mathrm{kPa},{ }^{69}$ which it did not during our measurements. Because before each single measurement calibration was performed the effect caused by a slightly different connection of the tubes to the pneumotachometers could be ignored. Furthermore, changes in temperature inside the spirometer during heating of the pneumotachometers could be neglected. After being heated by the pneumotachometer to temperatures above $25^{\circ} \mathrm{C}$, air cools down to room temperature by the time it reached the spirometer, which was $160 \mathrm{~cm}$ further in the circuit. The maximum temperature $23.2^{\circ} \mathrm{C}$ measured at $80 \mathrm{~cm}$ behind the pneumotachometer, where the thermometer was located, proved that the air equilibrated quickly to room temperature $\left(22^{\circ} \mathrm{C}\right)$. When, for example at a NICU, the oxygen concentration in inspiratory air is increased, the viscosity of this gas mixture increases and thereby causes the pneumotachometers to overestimate flow rates. ${ }^{97}$ This effect was confirmed by our results presenting a progressive measurement error at increasing oxygen concentrations. Because differences between pneumotachometers were relatively small and because they were not considered as the purpose of this study, a thorough discussion concerning the potential causes did not seem appropriate.

In equipment based on differential pressure, like pneumotachometers, turbulence can cause a considerable error, thereby overestimating flow rates. Frey et $\mathrm{al}^{73}$ indicated that the physical properties of most pneumotachometers usually cause a non-linear relationship between the applied and measured signals. According to Yeh et al. ${ }^{269}$ flow-conductance characteristics of differential pressure pneumotachometers are non-linear. Finucane et al. ${ }^{67}$ observed a non-linearity in screen pneumotachometers as well as in Fleisch type pneumotachometers. Our data showed that flow errors in all pneumotachometers increased considerably when flow rates were progressively higher than calibration flow rate. Pneumotachometers underestimated at flow rates lower than calibration flow of $3 \mathrm{~L} / \mathrm{min}$.

Although constant in the absolute way, this small error increased relatively at lower flow rates. Noise in the recorded signals probably caused the most important part of this measurement error. Although the used pneumotachometers should be linear within the flow range measured, non-linearity of the pneumotachometers within this range was, to our opinion, the most plausible explanation for the error pattern that was found. Differences between pneumotachometers were again relatively small and were therefore not considered for discussion.

The correction factors, following the multiple regression equations for each heated and unheated pneumotachometer separately, reduced the measurement errors significantly in all pneumotachometers. Maximal error ranges were reduced by more than 50 per cent in most pneumotachometers. Peak flow rates in preterm neonates might exceed $6 \mathrm{~L} / \mathrm{min}$ in some cases. If we assume that flow rates in preterm infants 
varied between 0 and $6 \mathrm{~L} / \mathrm{min}$, according to our results, a measurement error of \pm 9 per cent could be expected when no correction was performed $( \pm 5$ per cent because of the oxygen effect plus \pm 4 per cent caused by the flow rate effect). After correction for oxygen and flow effects a residual effect of \pm 2 per cent caused by variance in oxygen concentration and flow rate could be expected within a flow range of $0-6$ $\mathrm{L} / \mathrm{min}$. The pneumotachometers we tested are predominantly used in research settings to measure lung function (compliance, resistance, forced expiratory flow rates, etc.) in preterm infants. The role of lung function testing in improving ventilator adjustments, and diagnosis and prognosis of respiratory morbidity in neonates has been investigated increasingly during recent years. Consequently, lung function measures like respiratory resistance, which is highly flow dependent, will become more accurate after correction. Some mechanical ventilators use comparable pneumotachometers. When the same pneumotachometers are used as described above the built-in corrections for BTPS might be extended with corrections for oxygen concentrations and flow rates based on our results. Integral application of the regression equations will be necessary for a reliable correction during conditions of changing flow.

Correction factors were based upon measurements with dry gases at $22^{\circ} \mathrm{C}$, which were sufficiently constant during our measurement procedures. Because of their opposite effects on the viscosity of a gas a simultaneous increase of humidity and temperature from dry air in the hospital circuit during calibration procedures (relative humidity approximating 0 per cent at $22^{\circ} \mathrm{C}$ ) to a humidified and warmed environment (relative humidity 95 per cent at $37^{\circ} \mathrm{C}$ ) will result in a measurement error of approximately 2.9 per cent ( 4.1 per cent caused by the increase of temperature and 1.2 per cent caused by the increased humidity). ${ }^{17}$ This implicates that besides corrections for the effects of oxygen concentrations and flow rates humidity and temperature effects should be corrected for when flow measurements are performed at the NICU.

\subsection{Conclusions}

Our data showed that measurement errors in pneumotachometers were significantly affected by both flow rate and oxygen concentration. Therefore, it is necessary to calibrate with the actual oxygen concentrations and flow rates before starting a measurement in clinical or experimental settings. However, frequent calibration procedures during changes in gas composition in the clinical setting is often not practical, e.g. in case of increasing oxygen need in the neonate. For this reason we calculated regression equations to correct flow readings during changing conditions, which significantly reduced the measurement errors. 
This means that after a calibration at 0 and $3 \mathrm{~L} / \mathrm{min}$ and 60 per cent oxygen reliable flow measurements can be achieved in the appropriate flow range and at all oxygen concentrations with the described neonatal pneumotachometers. In practice quick corrections can be performed using polynomials as shown in Figure 4. Further investigation is needed to extend the applicability of the correction factors in humidified and warmed conditions. 



\section{CHAPTER 4}

Chest radiograph scores in preterm infants: interobserver agreement and relation with respiratory function

Yvonne Snepvangers

Huibert Burger

Peter de Winter

Bert Arets

Eric Beek

Kors van der Ent 



\subsection{Abstract}

To evaluate scoring systems for chest radiographs, we determined the interobserver agreement and relation with lung function during the first week of life in ventilated preterm infants. Three independent observers examined chest radiographs by applying radiological scores according to Lischka, Yuksel, Greenough, Toce, and Giedion at postnatal days 2 and 7. Kappa-statistics were used to assess interobserver agreement. By means of regression analysis mean scores and individual radiological scores of three observers were studied in relation to ventilation and oxygenation indices, and respiratory system resistance and compliance. Forty-eight radiographs were evaluated at day 2 , and 17 at day 7 . All scoring systems showed kappa values equal or lower than 0.5. Regression analysis revealed no significant associations between radiological scores and ventilator requirements or respiratory mechanics. We conclude that in ventilated preterm infants radiological scoring systems showed poor interobserver agreement and that they were not related to current respiratory function.

\subsection{Introduction}

Several radiological scoring systems have been developed to describe chest radiographs in relation to pulmonary disease and respiratory course in preterm neonates. Yuksel et al., ${ }^{277}$ Greenough et al. ${ }^{95}$ and Toce et al. ${ }^{242}$ developed scores to predict the respiratory outcome of preterm infants. Scores of Lischka et al. ${ }^{154}$ and Giedion et al. ${ }^{86}$ were developed to indicate the severity of respiratory distress syndrome (RDS). The scores are mainly based on characteristics like lung volume, air bronchograms, reticulogranular pattern, and the presence of cysts that may point to RDS (Figure 1) or a beginning chronic lung disease of the newborn (CLD; Figure 2).

At the neonatal intensive care unit (NICU), chest radiography is regularly used to check for pulmonary problems. In the multidisciplinary NICU setting these radiographs are often judged by more than one physician, e.g. a neonatologist, radiologist or pulmonologist. Good interobserver agreement is a prerequisite for applying scoring systems in general practice and as an outcome parameter in clinical studies.

In older children, for example with cystic fibrosis, a good correlation has been observed between radiological scores and lung function. ${ }^{169}$, 203 Such associations have been studied only scarcely in ventilated preterm infants. ${ }^{154}$

In order to evaluate the clinical value of chest radiographs in preterm ventilated infants, we studied the interobserver agreement of radiological scoring systems and whether these scoring systems related to functional markers of the respiratory condition at that time. 


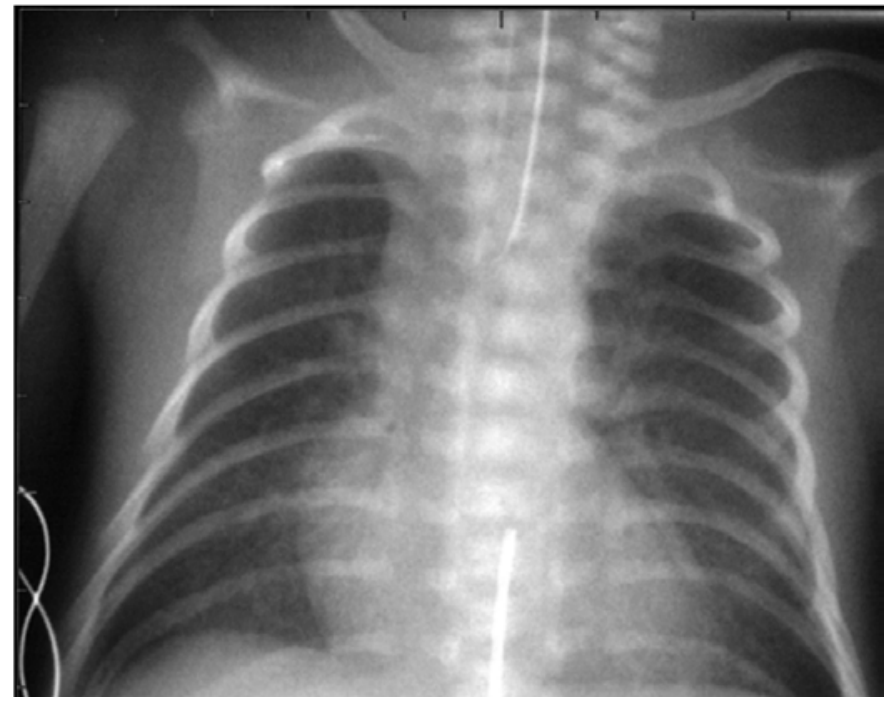

Figure 1. Chest radiograph of boy at day 2 of life. Birth weight: 1575 g. Gestational age: 33 wk +5 d. Surfactant was administered once at 6 hours after birth. Current ventilator requirements: synchronized intermittent mandatory ventilation with a peak inspiratory pressure of $17 \mathrm{~cm} \mathrm{H}_{2} \mathrm{O}$, a positive end expiratory pressure of $5 \mathrm{~cm} \mathrm{H} \mathrm{H}_{2} \mathrm{O}$, a frequency of $40 / \mathrm{min}$, and no supplemental oxygen.

Scores of three observers:

Lischka scores:

$4-9-4$; mean \pm S.D.: $5.7 \pm 2.9$

Yuksel scores:

$4-4-4$; mean \pm S.D.: $4.0 \pm 0.0$

Greenough scores:

$2-2-2 ;$ mean \pm S.D.: $2.0 \pm 0.0$

Toce scores:

Giedion scores:

$2-2-3$; mean \pm S.D.: $2.3 \pm 0.6$

$0-1-0$; mean \pm S.D.: $0.3 \pm 0.6$

\subsection{Patients and methods}

This study was part of a large longitudinal study investigating lung function in ventilated preterm infants. Infants of less than 37 weeks gestation born between July 1999 and August 2000 at the NICU of the University Medical Centre Utrecht, The Netherlands, or admitted to this centre within 24 hours after birth, were recruited. For inclusion, mechanical ventilation had to be started within 24 hours after birth. Infants with congenital anomalies, like multiple external dysmorphias, skeleton abnormalities, and hernia diaphragmatica were excluded from the study. Lung function tests were 


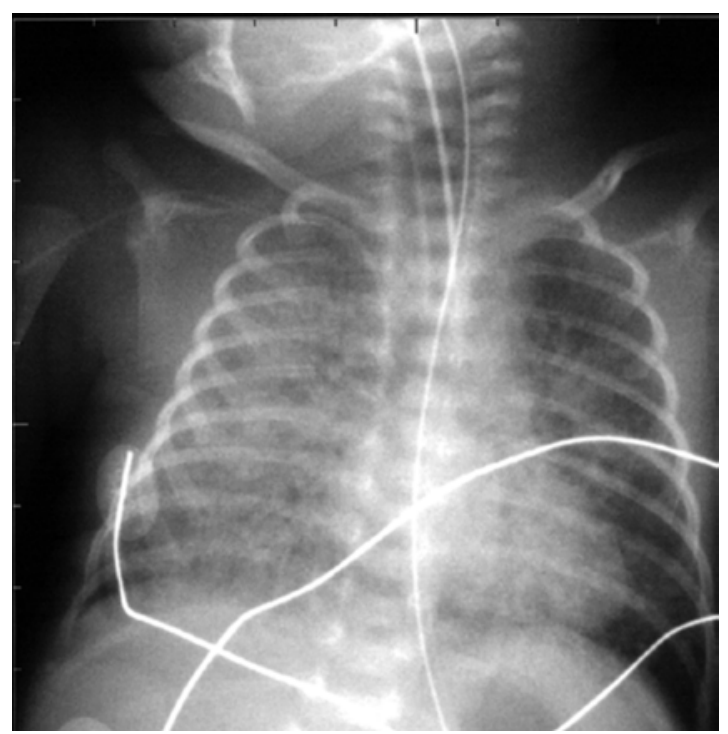

Figure 2. Chest radiograph of boy at day 6 of life. Birth weight: 1060 g. Gestational age: 28 wk +4 d. Surfactant was administered twice during the first days of life. Current ventilator requirements: synchronized intermittent mandatory ventilation with a peak inspiratory pressure of $16 \mathrm{~cm} \mathrm{H}_{2} \mathrm{O}$, a positive end expiratory pressure of $5 \mathrm{~cm} \mathrm{H} \mathrm{H}_{2} \mathrm{O}$, a frequency of $40 / \mathrm{min}$, and an inspired oxygen fraction of 0.27 .

Scores of three observers:

Lischka scores:

$$
\begin{array}{cl}
10-9-10 ; \text { mean } \pm \text { S.D.: } & 9.7 \pm 0.6 \\
10-15-11 ; \text { mean } \pm \text { S.D.: } & 12.0 \pm 2.7 \\
6-8-6 \text {; mean } \pm \text { S.D.: } & 6.7 \pm 1.2 \\
7-7-5 ; \text { mean } \pm \text { S.D.: } & 6.3 \pm 1.2
\end{array}
$$

$\begin{array}{ll}\text { Toce scores: } & 7-7-5 \text {; mean } \pm \text { S.D.: } 6.3 \pm 1.2 \\ \text { Giedion scores: } & 1-4-3 \text {; mean }+ \text { S.D.: } 2.7 \pm 1.5\end{array}$

performed at days 2 (24-48 hours) and 7. Only infants, in whom digital chest radiographs of day 2 (24-48 hours) or day $7 \pm 1$ were clinically indicated, were selected for further analysis. The study was approved by the Medical Ethics Review Committee of our centre and parental informed consent was obtained.

Mechanical ventilation was started as a consequence of respiratory distress, when, despite supplemental oxygen or continuous positive airway pressure, the inspiratory oxygen fraction $\left(\mathrm{FiO}_{2}\right)$ remained above 0.7 , the partial arterial carbon dioxide pressure $\left(\mathrm{PaCO}_{2}\right)$ exceeded $55 \mathrm{~mm} \mathrm{Hg}$, acidosis persisted with $\mathrm{pH}$ decreasing below 7.25, or when serious apnoeas existed. Ventilator setting was adjusted to keep the arterial 
oxygen saturation of haemoglobin between $90-95 \%$ and the $\mathrm{PaCO}_{2}$ between $40-45$ $\mathrm{mm} \mathrm{Hg}$. For mechanical ventilation, one of the following infant respirators was used: Stephanie (Stephan, Gackenbach, Germany), Bear BP2001 (Bear, Palm Springs, CA, USA), and Bourns BP200 (Bourns, Inc., Riverside, CA, USA). When the PIP exceeded $25 \mathrm{cmH}_{2} \mathrm{O}$, conventional ventilation was switched to high frequency oscillatory ventilation (HFOV).

Table 1. Characteristics of radiological scores

\begin{tabular}{|c|c|}
\hline Radiological scores & Characteristics \\
\hline Lischka $^{154}$ & $\begin{array}{l}\text { Pattern (few streaks to completely reticulogranular: score } 1,2,3 \text { ) } \\
\text { Size of specific pattern (single area to > two third of lungs: score } 1,2,3 \text { ) } \\
\text { Density of pattern (<liver to homogeneous to liver: score } 1,2,3) \\
\text { Air distribution (normal to extra-alveolar presence of air: score } 1,2,3 \text { ) }\end{array}$ \\
\hline Yuksel 277 & $\begin{array}{l}\text { Quality of X-ray (bad or good: score } 0,1 \text { ) } \\
\text { Intubated or not (score } 0,1) \\
\text { Lung volume } \\
\text { (configuration diaphragm/ribs: normal, de-, increased: score } 0,1,2) \\
\text { Number of posterior ribs }(<14,14-16,>16 \text { : score } 0,1,2 \text { ) } \\
\text { Opacification (not present, perihilar/regional, complete: score } 0,1,2,3 \text { ) } \\
\text { Air bronchogram(s) present in outer third of lungs or not (score } 0,1 \text { ) } \\
\text { Interstitial changes (none to present in four zones: score } 0,1,2,3,4) \\
\text { Emphysematic changes (none to bullae present: score } 0,1,2) \\
\text { Cystic changes (none to multiple: score } 0,1,2 \text { ) } \\
\text { Size of cysts (not present to >one third of lungs: score } 0,1,2)\end{array}$ \\
\hline Greenough ${ }^{95}$ & $\begin{array}{l}\text { Number of posterior ribs }(<14,14-16,>16 \text { : score } 0,1,2) \\
\text { Interstitial changes (none to present in four zones: score } 0,1,2,3,4) \\
\text { Cystic changes (none to multiple: score } 0,1,2 \text { ) }\end{array}$ \\
\hline Toce $^{242}$ & $\begin{array}{l}\text { Number of posterior and anterior ribs right sided } \\
\quad(<14,14-16,>16 \text { : score } 0,1,2) \\
\text { Heart figure size (normal to enlarged: score } 0,1,2) \\
\text { Interstitial changes (none to considerable: score } 0,1,2) \\
\text { Emphysematic changes (none to bullae present: score } 0,1,2) \\
\text { Subjective judgement (mild to seriously abnormal: score } 0,1,2)\end{array}$ \\
\hline Giedion $^{86}$ & $\begin{array}{l}\text { (0) No signs of pulmonary disease } \\
\text { (1) Reticulogranular pattern } \\
\text { (2) Reticulogranular pattern and air bronchogram } \\
\text { (3) Reticulogranular pattern, air bronchogram and indistinct heart outline } \\
\text { (4) Totally white appearance }\end{array}$ \\
\hline
\end{tabular}


Digital chest radiographs were obtained at maximal inspiratory level in supine position. Five radiological scores for preterm neonates were used: Lischka, ${ }_{154}^{154}$ Yuksel, 277 Greenough, ${ }^{95}$ Toce $^{242}$ and Giedion ${ }^{86}$ (Table 1). Two paediatric pulmonologists (B.A. and K.v.d.E.) and one paediatric radiologist (E.B.) independently evaluated the images with the use of the five scoring systems. They were unaware of the infants' clinical condition.

With each radiograph, the oxygenation index (= (mean airway pressure $\left.* \mathrm{FiO}_{2}\right) /$ $\mathrm{PaO}_{2}$ ), a measure for diffusion capacity of the lungs, and the ventilation index (= (ventilator frequency $*$ peak inspiratory pressure $* \mathrm{PaCO}_{2}$ ) / 1000), ${ }^{197}$ a measure for alveolar ventilation, were determined. High indices imply poor pulmonary gas exchange. Time of assessment was equal to the time at which respiratory function measurements were performed.

Measurements of passive respiratory system resistance $\left(R_{\mathrm{rs}}\right)$ and compliance $\left(C_{\mathrm{rs}}\right)$ were performed at days 2 and 7 of life. Since measurements were only performed in intubated infants, fewer infants participated at day 7 because they did not longer require mechanical ventilation. $R_{\mathrm{rs}}$ and $C_{\mathrm{rs}}$ were assessed according to the single breath occlusion technique (SBT) described by Le Souef.68,150 A heated Lilly-type pneumotachometer $(8410 \mathrm{~A}$, linear range $0-10 \mathrm{~L} / \mathrm{min}$, dead space $1.3 \mathrm{ml}$, resistance 0.96 $\mathrm{kPa} / \mathrm{L} \cdot \mathrm{s}$ at $5 \mathrm{~L} / \mathrm{min}$; Hans Rudolph Inc., Kansas City, MO, USA) was placed between endotracheal tube and ventilator tubes. A pressure transducer (Honeywell, type 163PC01D75, Morristown, NJ, USA) and an IBM compatible personal computer with a Pentium processor running the software package Respiratory Analysis System Program (Version 007.17, Physio Logic, High Clere, UK) were used to record signals at a sampling rate of $125 \mathrm{~Hz}$ using a 12 bit analogue-to-digital converter (LabMaster DMA, Scientific Solutions, Mentor, OH, USA). Mouth pressure and flow signals were obtained after calibration before each single measurement. For flow calibration, a calibrated rotameter (air 0-15 L/min, Medec, Aalst, Belgium) was used followed by a volume calibration using a precision syringe of $50 \mathrm{ml}$ containing the actual gas mixture. For pressure calibration, a pressure transducer tester (Veri-Cal 650-900, UT Medical Products Inc., Midvale, UT, USA) was used. Leaks in the system or around the endotracheal tube were easily detected as mouth pressure decreased gradually during occlusion. With gentle manual compression of the trachea present leaks were minimized. Persisting tube leaks causing a decrease in occlusion pressure of $0.02 \mathrm{kPa}$ or more were excluded from further analysis. ${ }^{45,220}$ During occlusion, activity of inspiratory muscles is inhibited via stimulation of the airway receptors inducing the Hering-Breuer reflex. Breath analysis was considered unreliable, when no muscle relaxation was achieved. Muscle relaxation was assumed to be present when (1) during a short occlusion period of less than 0.5 second mouth pressure did reach a plateau for at least 0.1 seconds with a standard deviation (SD) of $0.01 \mathrm{kPa}$ or less, and (2) the expiration part of the flow volume curve was linear for at least 50 per cent of the volume to be expired. As a criterion for linearity, a correlation coefficient of 0.98 or 
Neonatal chest radiograph scores

more after exclusion of the final 5 per cent of the expired volume had to be reached. At least five occluded breaths meeting these criteria were used to calculate a mean value for both respiratory mechanics in each single measurement. These criteria complied with the European Respiratory Society (ERS) recommendations. ${ }^{80}$ No correction was made for endotracheal tube resistance. ${ }^{164}$

\subsubsection{Statistics}

Kappa-statistics were calculated to assess the chance-corrected proportional agreement between observers. ${ }^{6}$ For each possible pair of observers (three in total) a kappa was calculated. Subsequently, the obtained kappas were averaged for each score at days 2 and 7 separately. Kappa values lower than 0.5 represent poor agreement. ${ }^{6}$ To assess whether interobserver variability affected our results individual as well as mean scores of the three observers were studied in relation to clinical and lung function variables. Using regression analyses, associations between radiological scores as independent variables and the oxygenation and ventilation indices, $R_{\mathrm{rs}}$ and $C_{\mathrm{rs}}$ as dependent variables were studied. The oxygenation index and $R_{\mathrm{rs}}$ were transformed by taking the natural logarithm, because of their deviation from the normal distribution. Data were analysed for days 2 and 7 separately.

\subsection{Results}

Fifty-one infants were initially included in the study. In three of them respiratory function measurements were unreliable according to ERS criteria. Hence, at the second day of life, results of 48 infants were evaluated. In 17 of 51 infants both chest radiographs and respiratory function results were available at day 7 . Fourteen of these 17 patients had been included for evaluation at day 2. Patient characteristics are presented in Table 2.

Radiological scoring systems were categorized before kappas were calculated. Kappa-statistics could not be calculated when constants were present or when complete categories were not scored by one or two observers. The Yuksel score was categorized into four categories: 0-5/6-10/11-15/16-20. At day 2, the mean kappa of the three pairs of observers was 0.48 and at day 7 this was 0.11 . The Greenough score was categorized into three categories: $0-2 / 3-5 / 6-8$. The mean kappa of the Greenough score was 0.18 at day 2 and 0.20 at day 7 . The Toce score was categorized into three categories: $0-2 / 3-6 / 7-10$ and its mean kappa was 0.20 at day 2 and 0.50 at day 7. The Lischka score was categorized into three categories: 4-6/7-9/10-12 and its mean kappa was 0.01 at day 2 , and 0.14 at day 7 . Finally, the Giedion score was categorized into three categories: 0/1-2/3-4 and its mean kappa was -0.04 at day 2 and 0.26 at day 7 . 
Table 2. Patient characteristics.

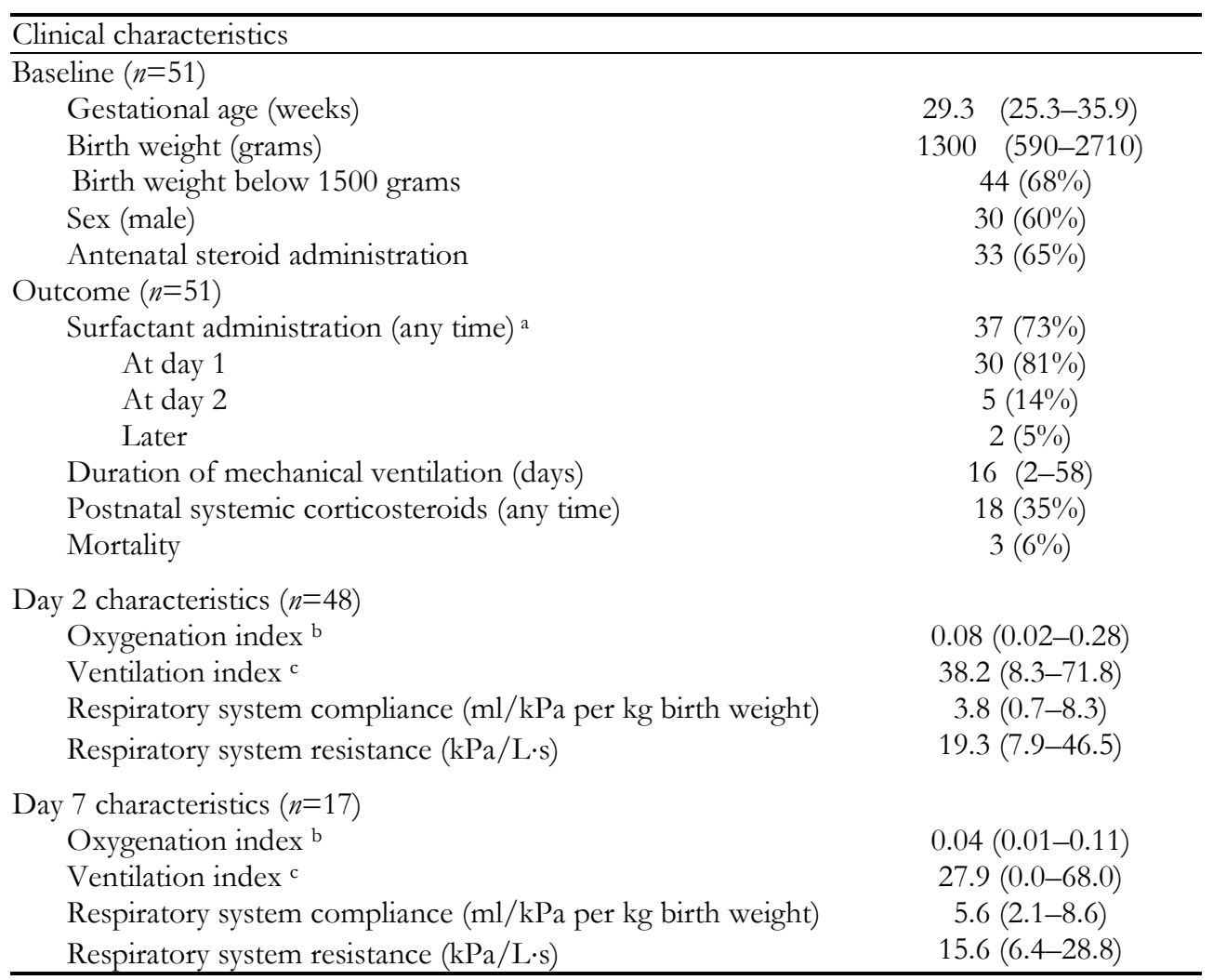

Data presented as mean (range) or number (percentage).

${ }^{a}$ Criteria for surfactant administration are explained in the Methods section.

$\mathrm{b}$ Oxygenation index $=($ mean airway pressure $*$ inspired oxygen fraction $) / \mathrm{pO}_{2}$.

c Ventilation index $=$ (ventilator frequency $*$ peak inspiratory pressure $\left.* \mathrm{pCO}_{2}\right) / 1000$. One infant receiving continuous positive airway pressure was indexed 0 concerning the ventilation index.

No statistically significant associations were found between the mean radiological scores of the three observers and any of the respiratory function measures neither at day 2 (Figure 3) nor at day 7. With respect to individual radiological scores, only two statistically significant associations were found: (1) between respiratory system resistance and the Toce score by one observer at day 7 ( $\beta$ per radiological score unit $=0.126 ; 95 \%$ confidence interval $(\mathrm{CI}), 0.017$ to $0.234 ; p=0.027)$, and (2) between oxygenation index and Lischka score by another observer at day $2(\beta$ per radiological score unit $=0.049 ; 95 \%$ CI, 0.003 to 0.096; $p=0.037$ ). Besides these two exceptions, results concerning individual radiological scores as well as mean scores at day 7 were similar to the results of the mean scores at day 2, presented in Figure 3. 

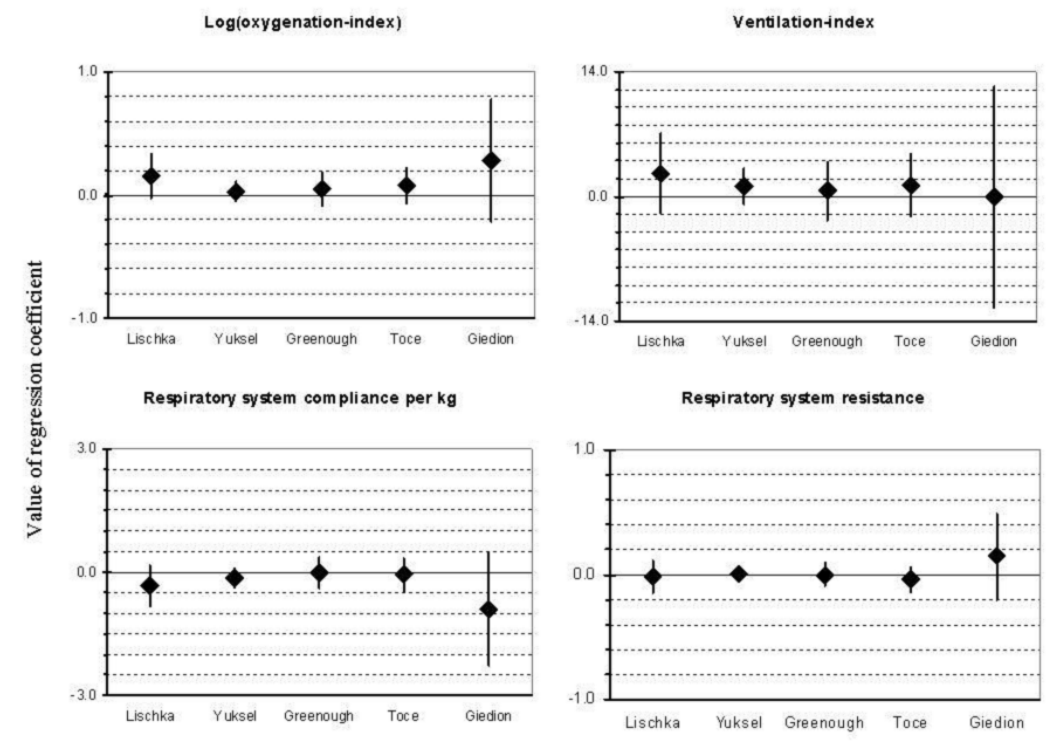

Radiological scoring systems used as independents in each regression model

Figure 3. Results of regression analysis exploring the associations between the mean radiological scores of three observers (independent variables) and respiratory function variables (dependent variables) assessed at the second day of life. Regression coefficients ( $\beta$ ) are presented with their $95 \%$ confidence intervals. Results concerning individual radiological scores and scores at day seven were similar to these results.

\subsection{Discussion}

Radiological scoring systems used to evaluate neonatal chest radiographs showed poor interobserver agreement. Furthermore, none of the studied radiological scoring systems was associated with respiratory function. Although in earlier studies radiological scoring systems were developed and evaluated, this is the first study to evaluate associations between several of these scoring systems and respiratory function measures during the first week of life in ventilated preterm infants.

The poor interobserver agreement is of clinical importance. Our data show that experienced physicians remarkably differ in their interpretations of radiological findings. This argues the importance of the presence of actual chest radiographs during clinical discussions, since individual physicians cannot judge chest radiographs from oral or written reports of a colleague. Our findings also question the value of 
using scoring systems as an endpoint in clinical studies. Interobserver variability may be decreased in computer-assisted analysis of chest radiographs in preterm infants. ${ }^{50}$

We were not able to find any relationship between radiological scores and lung function parameters. Beside the lack of inter-observer agreement this may be due to several other factors. Firstly, radiographs may accentuate different aspects of respiratory pathology compared to respiratory function. Clear radiological abnormalities, like singular cysts, may only have trivial effects on respiratory condition. On the contrary, radiologically invisible abnormalities, like bronchospasms, may have major effects on current respiratory condition. Factors affecting the radiological lung appearance as well as extrapulmonary factors affecting ventilation or oxygenation indices, like cardiovascular shock or improper placement of the arterial catheter, may cause a poor match between radiological findings and ventilator requirements.

A second reason for the lack of agreement between lung function and chest radiograph scores may be that the used scoring systems were not developed for children at this age. The Yuksel score, partly based on aspects related to persistent pulmonary problems, like haziness and cystic abnormalities, was originally developed to be used at 1 month of age. The Greenough score should be used at 28 days of age and consisted of similar radiological items, including interstitial abnormalities and lung volume. Toce's score was meant to be applied at 21 days of age to assess the severity of CLD. Yuksel et al. stated that their score was also applicable at day 7 of life 276 and because Greenough and Toce scores were very similar, we decided to include these scoring systems as well. Furthermore, after unravelling the Yuksel, Greenough and Toce scores, it appeared that they consisted partly of radiological aspects that may already be seen from the second day of life, like interstitial abnormalities and in- or decreased lung volume.

Furthermore, ventilator conditions may directly affect the radiological lung appearance, obscuring potential associations between radiological and functional factors as well. ${ }^{86}$ Additionally, limitations of the neonatal respiratory function measurements, ${ }^{150}$ especially in intubated preterm infants, may obscure potential relationships as well. ${ }^{228}$ However, by using strict criteria recommended by the ERS, validity of neonatal respiratory function results was acceptable. Although endotracheal tube resistance is known to substantially affect neonatal $R_{\mathrm{rs}}$ values, we decided not to correct for endotracheal tube resistance. Previous studies showed that correction does not guarantee a better approximation of $R_{\mathrm{rs}}{ }^{152,164}$

In contrast to our findings, Lischka and colleagues described significant associations between their score and $C_{\mathrm{rs}}$ within 24 hours after birth. ${ }^{154}$ Others described correlations between Giedion scores and parameters of lung function ${ }^{43,124}$, However, these studies were performed before exogenous surfactant therapy had been widely introduced. Many infants in our study did receive exogenous surfactant at the first day of life, which is known to affect both radiological and functional aspects, decreasing RDS severity ${ }^{32}$ and increasing $C_{\mathrm{rs}}$ directly. ${ }^{45}$ 
In conclusion, radiological scoring systems used to evaluate neonatal chest radiographs showed poor interobserver agreement in ventilated preterm infants and were not associated with functional parameters of respiratory condition. The use of these scoring systems as an indicator of the current respiratory condition during the first week of life is not justified. 


\title{
CHAPTER 5
}

\section{Early prediction of clinical respiratory course in preterm ventilated infants}

\author{
Yvonne Snepvangers \\ Huibert Burger \\ Peter de Winter \\ Bert Arets \\ Eric Beek \\ Kors van der Ent
}

Submitted 



\subsection{Abstract}

Aim: To predict prolonged mechanical ventilation ( $\geq 10$ days) and need of postnatal steroid therapy in preterm ventilated infants early after birth in an attempt to select high-risk infants in order to improve implementation of practical preventive measures and increase research possibilities.

Methods: Preterm infants of $<37$ weeks gestation, mechanically ventilated from day 1 , were recruited. Chest radiographs and lung function tests performed at day 2 were evaluated together with demographic information by logistic regression analysis and receiver-operating characteristic (ROC) curves.

Results: In 48 preterm infants prolonged mechanical ventilation was reliably predicted by radiological signs of haziness and hyperinflation, and the respiratory system resistance. Gestational age, haziness and respiratory system resistance accurately predicted postnatal need of steroid therapy. Both prediction models had areas under the ROC curves of $>0.80$ after dichotomization of independent variables. Convenient scores based on regression coefficients roughly indicated infants to three different risk levels for each outcome.

Conclusion: Preterm infants at high risk of prolonged mechanical ventilation and postnatal need of steroid therapy could be selected at 48 hours after birth using prediction models based upon clinical, radiological and lung mechanical information. Identifying preterm infants at risk of separate aspects of respiratory outcome may have important advantages in serving separate goals.

\subsection{Introduction}

The introduction of prenatal corticosteroids and postnatal exogenous surfactant treatment in perinatal care, probably have played a major role in the observed trend of decreasing severity of respiratory distress syndrome (RDS) during the past decades. ${ }^{32}$ Nevertheless, the risk of developing chronic lung disease of the neonate (CLD) has remained high and may even have increased. ${ }^{58}$ The risk to develop acute respiratory morbidity decreases with increasing gestational age, although RDS may develop until 37 weeks post-conceptional age up to even 5 per cent of the infants born after this age. ${ }^{138}$

Since pathophysiologic processes causing chronic lung disease (CLD) in these infants may start as early as in utero, ${ }^{211,272}$ early intervention ${ }^{101}$ may be one of the most effective ways to prevent pulmonary morbidity. During the first 24 hours after birth, signs of a wet lung may obscure underlying pulmonary pathology. At the second day of life signs of RDS generally become more apparent. Preventive measures, like minimizing ventilator conditions ${ }^{268}$ or early corticosteroid administration, probably 
give most benefit when applied as early as possible. Hence, the ideal timing to predict clinical respiratory course in these infants may be between 24 and 48 hours of life.

Most infants with RDS are being ventilated for less than 1 week, since clinical deterioration generally reaches a peak around the third day of life. 138 Hence, ventilator dependency of $\geq 10$ days would reliably select those infants with ongoing pulmonary problems. On the other hand, infants in need of late steroid therapy are most interesting subjects to investigate the effect of early steroid therapy in lower doses or with less potent steroid drugs, as has been implicated by the Cochrane Review. ${ }^{101}$

We intended to construct two prediction models to select high-risk infants for prolonged mechanical ventilation ( $\geq 10$ days) and postnatal systemic steroid treatment in ventilated preterm infants. To include as many infants at risk as possible, all preterm infants with respiratory distress requiring mechanical ventilation within 24 hours after birth born after a gestational age of $<37$ weeks were studied.

\subsection{Materials and methods}

\subsubsection{Patients}

This study was part of a large longitudinal study investigating respiratory function in ventilated preterm infants. Infants of less than 37 weeks gestation born between 28 July 1999 and 31 August 2000 at the University Medical Centre Utrecht, The Netherlands, or admitted to this centre within 24 hours after birth, were recruited into the study. For inclusion, mechanical ventilation had to be started within 24 hours after birth (i.e. day 1). Infants with congenital anomalies were excluded from the study. For the longitudinal study, respiratory function tests were performed at days $1(0-24$ hours), 2, 3, 7 and just before extubation. Only infants, in whom digital chest radiographs of day 2 (24-48 hours) were clinically indicated, were selected for further analysis. The study was approved by the Medical Ethics Review Committee of the University Medical Centre Utrecht and informed parental consent was obtained for each infant.

Infants were intubated when, despite measures of supplemental oxygen and/or nasal continuous positive airway pressure (CPAP), an oxygen supply of $>70$ per cent remained necessary, partial pressure of arterial carbon dioxide $\left(\mathrm{PaCO}_{2}\right)$ was $>55 \mathrm{~mm}$ $\mathrm{Hg}$ or arterial $\mathrm{pH}$ was $<7.25$. The inner diameter of the endotracheal tubes varied from $2.5 \mathrm{~mm}$ to $3.0 \mathrm{~mm}$ with a total length between 11.0 and $18.0 \mathrm{~cm}$. The ventilators that were used were: Stephanie (Stephan, Gackenbach, Germany), Bear BP2001 (Bear, Palm Springs, CA, USA), and Bourns BP200 (Bourns, Inc., Riverside, CA, USA). At the discretion of the attending neonatologist, who was not involved in the study, ventilator conditions were adjusted to keep the arterial oxygen saturation of haemoglobin $\left(\mathrm{SaO}_{2}\right)$ between $90-95$ per cent and $\mathrm{PaCO}_{2}$ at $40-45 \mathrm{~mm} \mathrm{Hg}$. When 
radiological signs of $\operatorname{RDS}^{86}$ were present while being mechanically ventilated, exogenous surfactant (natural bovine surfactant: Alvofact ${ }^{\circledR}$, Boehringer, Ingelheim, Germany) was administered at a dose of $50 \mathrm{mg} / \mathrm{kg}$ according to the following limits of positive inspiratory pressure (PIP) and the inspiratory fraction of oxygen $\left(\mathrm{FiO}_{2}\right)$ : a PIP $<20 \mathrm{cmH}_{2} \mathrm{O}$ with a $\mathrm{FiO}_{2}>0.60$ and a PIP $\geq 20 \mathrm{cmH}_{2} \mathrm{O}$ with a $\mathrm{FiO}_{2}>0.45$. When the PIP exceeded $25-\mathrm{cmH}_{2} \mathrm{O}$ conventional ventilation was switched to high frequency oscillatory ventilation (HFOV). In case of (1) a postnatal age of >7-14 days, (2) ventilator dependency with an increased inspired oxygen fraction of $>0.30$, (3) radiological signs of CLD (including stage I abnormalities) described by Northway et al. ${ }^{188}$, and (4) no potential to decrease ventilator conditions for 72 hours, systemic hydrocortisone therapy (Solu-Cortef ${ }^{\circledR}$, Pharmacia \& Upjohn, Pharmacia Corporation, NJ, USA) was started. Hydrocortisone therapy was initiated at the discretion of the attending neonatologist, who was not involved in the study. This occurred most often in the second week of life with $5 \mathrm{mg} / \mathrm{kg}$ per day in four divided doses. The amount per dose as well as the number of doses decreased slowly at intervals of 5 days reaching a total of 22 days of therapy. In the absence of respiratory improvement, steroid treatment was either prolonged or repeated.

\subsubsection{Predictors}

We studied the predictive value of seven, early available, variables to predict prolonged ventilator need ( $\geq 10$ days) and need of postnatal steroid treatment. These variables referred to clinical, radiological and lung function aspects, of which the latter two were assessed between 24 and 48 hours after birth. Clinical variables included gestational age $(\mathrm{GA})$, prenatal steroid administration to stimulate lung maturation and premature rupture of the membranes (PROM). Radiological variables included the presence of haziness (increased density: $0=$ no haziness; $1=$ regionally localized or around hilus; 2 regionally and around hilus; $3=$ total haziness) and the number of posterior ribs bilaterally $(0=$ less than 14 ribs; $1=$ between 14 to 16 ribs; $2=$ more than 16 ribs) as a measure of hyperinflation. Digital chest radiographs were obtained at maximal inspiration in infants in supine position. These chest radiographs were scored independently by a paediatric pulmonologist and a paediatric radiologist, both unaware of the clinical course of the infants. The mean score of both physicians was calculated for each variable, since this corresponded with current clinical practice at most NICUs, where chest radiographs are judged by at least two physicians, a neonatologist and a radiologist. Lung function variables included respiratory system compliance $\left(C_{\mathrm{rs}}\right)$ and resistance $\left(\mathrm{R}_{\mathrm{rs}}\right)$. 


\subsubsection{Pulmonary Function Tests}

$R_{\mathrm{rs}}$ and $C_{\mathrm{rs}}$ were assessed between 24 and 48 hours of life according to the single breath occlusion technique (SBT) described by Le Souef.68,150 Sometimes measurements could not be performed, because of clinical instability of the patient or unavailability of qualified personnel. In case infants received HFOV, a switch to SIMV was required when the clinical condition allowed this. A heated Lilly-type pneumotachometer $(8410 \mathrm{~A}$, linear range $0-10 \mathrm{~L} / \mathrm{min}$, dead space $1.3 \mathrm{ml}$, resistance $0.94 \mathrm{kPa} / \mathrm{L} \cdot \mathrm{s}$ at $5 \mathrm{~L} / \mathrm{min}$; Hans Rudolph Inc., Kansas City, MO, USA) was placed between endotracheal tube and ventilator tubes. A pressure transducer (Honeywell, type 163PC01D75, Morristown, NJ, USA) and an IBM compatible personal computer with a Pentium processor running the software package Respiratory Analysis System Program (Version 007.17, Physio Logic, High Clere, Great Britain) were used to record signals at a sampling rate of $125 \mathrm{~Hz}$ using a 12 bit analogue-to-digital converter (LabMaster DMA, Scientific Solutions, Mentor, OH, USA). Mouth pressure and flow signals were obtained after calibration before each single measurement. For flow calibration, a calibrated rotameter (Air 0-15 L/min, Medec, Aalst, Belgium) was used followed by a volume calibration using a precision syringe of $50-\mathrm{ml}$ containing the actual gas mixture. For pressure calibration, a pressure transducer tester (Veri-Cal 650900, Utah Medical Products Inc., Midvale, UT, USA) was used. Leaks in the system or around the endotracheal tube were easily detected as mouth pressure decreased gradually during occlusion. With gentle manual compression of the trachea present leaks were minimized. Measurements with persisting tube leaks causing a decrease in occlusion pressure of $0.02 \mathrm{kPa}$ or more were excluded from further analysis. 45,220 During occlusion, activity of inspiratory muscles is inhibited via stimulation of the airway receptors inducing the Hering-Breuer reflex. Analyses were performed according to the European Respiratory Society (ERS) recommendations valid during the time of the measurements. ${ }^{61,80}$ Breath analysis was considered unreliable, when no muscle relaxation was achieved. Muscle relaxation was assumed to be present when (1) during a short occlusion period of less than 0.5 seconds mouth pressure did reach a plateau for at least 0.1 seconds during which pressure changes showed a standard deviation (SD) of $0.01 \mathrm{kPa}$ or less, and (2) the expiration part of the flow volume curve was linear for at least 50 per cent of the volume to be expired. As a criterion for linearity, a correlation coefficient of 0.98 or more after exclusion of the final 5 per cent of the expired volume had to be reached. At least five occluded breaths meeting these criteria were used to calculate a mean value for both respiratory mechanics at the second day of life. 
Table 1. Patient characteristics $(n=48)$.

\begin{tabular}{|c|c|}
\hline & $\begin{array}{c}\text { Absolute number/mean } \\
(\% / \text { range })\end{array}$ \\
\hline Baseline: & \\
\hline \multicolumn{2}{|l|}{ Gestational age (wk) } \\
\hline$<28$ & $15(31)$ \\
\hline $28-29$ & $16(33)$ \\
\hline $30-31$ & $9(19)$ \\
\hline $32-34$ & $7(15)$ \\
\hline $35-36$ & $1(2)$ \\
\hline \multicolumn{2}{|l|}{ Birth weight (g) } \\
\hline $500-999$ & $16(33)$ \\
\hline $1000-1499$ & $16(33)$ \\
\hline 1500-1999 & $14(29)$ \\
\hline $2000-2499$ & $2(4)$ \\
\hline Sex (male) & $28(58)$ \\
\hline 1-minute Apgar score & $4.8(0-9)$ \\
\hline 5-minute Apgar score & $7.7(3-10)$ \\
\hline Umbilical cord $\mathrm{pH}$ & $7.19(6.89-7.39)$ \\
\hline Maximum RDS classification ${ }^{a}$ & $3(1-4)$ \\
\hline Surfactant administration before 24 hours & $28(58)$ \\
\hline Surfactant administration between $24-48$ hours & $5(10)$ \\
\hline PDA (diagnosed before 48 hours) ${ }^{\mathrm{b}}$ & $13(27)$ \\
\hline $\mathrm{HFOV}$ at day $2^{\mathrm{c}}$ & $17(35)$ \\
\hline Duration of mechanical ventilation (days) & $16(2-58)$ \\
\hline Mortality & $3(6)$ \\
\hline \multicolumn{2}{|l|}{ Potential predictors } \\
\hline $\mathrm{PROM}^{\mathrm{d}}$ & $8(17)$ \\
\hline Prenatal corticosteroid administration & $31(65)$ \\
\hline Mean haziness score at radiographe & $1.1(0-3)$ \\
\hline Mean lung volume score at radiograph ${ }^{\mathrm{f}}$ & $1.7(1-2)$ \\
\hline$C_{\mathrm{rs}} / \mathrm{kg}(\mathrm{ml} / \mathrm{kPa})^{\mathrm{g}}$ & $3.8(0.7-8.3)$ \\
\hline $\mathrm{R}_{\mathrm{rs}} / \mathrm{kg}(\mathrm{kPa} / \mathrm{Las})^{\mathrm{h}}$ & $18.5(4.6-62.4)$ \\
\hline \multicolumn{2}{|l|}{ Outcome: } \\
\hline Prolonged mechanical ventilation ( $\geq 10$ days) & $27(56)$ \\
\hline Postnatal systemic steroid therapy (any time) & $17(35)$ \\
\hline
\end{tabular}

a Maximum score during the admission period according to the radiological Giedion gradation of respiratory distress syndrome $(0=$ no abnormalities and $4=$ total white appearance $) .86$

b Patent ductus arteriosus established by ultrasound performed by a paediatric cardiologist.

c High frequency oscillatory ventilation.

d Premature rupture of membranes ( 24 hours before delivery. ${ }^{\text {e }}$ Mean haziness score of two physicians according to 4 stages: $0=$ no haziness; $1=$ regionally localized or around hilus; $2=$ regionally and around hilus; $3=$ total haziness.

${ }_{\mathrm{f}}$ Mean lung volume score of two physicians according to the number of posterior ribs visible at the chest radiograph in 3 categories: $0=$ less than 14 ribs; $1=14$ to 16 ribs; $2=$ more than 16 ribs.

$\mathrm{g}$ Compliance of the respiratory system per kilogram birth weight according to the single breath occlusion technique. ${ }^{68}$

${ }^{\mathrm{h}}$ Resistance of the respiratory system per kilogram birth weight according to the single breath occlusion technique. 
Table 2. Univariate associations between a priori selected variables and outcome variables.

\begin{tabular}{|c|c|c|}
\hline \multirow[t]{2}{*}{ Independents (cut-off values) } & \multicolumn{2}{|c|}{ OR $(95 \% \text { CI })^{a}$} \\
\hline & $\begin{array}{l}\text { Prolonged } \\
\text { ventilation }\end{array}$ & $\begin{array}{c}\text { Need of } \\
\text { steroid therapy }\end{array}$ \\
\hline Gestational age $(<30 / \geq 30 \mathrm{wk})$ & $31.3(5.6-175.1)$ & $17.1(2.0-144.8)$ \\
\hline Prenatal corticosteroids (yes/no) & $0.6(0.2-1.9)$ & $0.3(0.1-1.1)$ \\
\hline $\mathrm{PROM}^{\mathrm{b}}$ (yes/no) & $1.4(0.3-6.5)$ & $3.9(0.8-18.9)$ \\
\hline Haziness $(0-3 \text { stages: } 0 / \geq 1)^{c}$ & $10.4(1.1-94.8)$ & $3.8(0.4-34.9)$ \\
\hline Lung volume $(0-2 \text { stages: } 0-1 / 2)^{\mathrm{d}}$ & $4.9(1.1-21.8)$ & $7.6(0.9-65.8)$ \\
\hline$C_{\mathrm{rs}} / \mathrm{kg}(\mathrm{ml} / \mathrm{kPa})(\leq 4 />4)^{\mathrm{e}}$ & $2.1(0.6-7.3)$ & $2.1(0.5-7.8)$ \\
\hline$R_{\mathrm{rs}} / \mathrm{kg}(\mathrm{kPa} / \mathrm{Las})(<15 / \geq 15)^{\mathrm{f}}$ & $5.7(1.6-20.0)$ & - \\
\hline$R_{\mathrm{rs}} / \mathrm{kg}(\mathrm{kPa} / \mathrm{Las})(<20 / \geq 20)$ & - & $9.6(2.3-40.2)$ \\
\hline \multicolumn{3}{|l|}{ adds ratio with $95 \%$ confidence interval. } \\
\hline \multicolumn{3}{|c|}{$\begin{array}{l}\text { b Premature rupture of membranes } \geq 24 \text { hours before delivery. } \\
\text { c Mean haziness score of two physicians according to } 4 \text { stages: } 0=\text { no haziness; } 1=\text { regionally localized or } \\
\text { around hilus; } 2=\text { regionally and around hilus; } 3=\text { total haziness. } \\
\text { d Mean lung volume score of two physicians according to the number of back ribs visible at the chest } \\
\text { radiograph in } 3 \text { categories: } 0=\text { less than } 14 \text { ribs; } 1=14 \text { to } 16 \text { ribs; } 2=\text { more than } 16 \text { ribs. } \\
\text { e Compliance of respiratory system per } \mathrm{kg} \text { birth weight according to the single breath occlusion } \\
\text { technique. } 6 \\
\text { f Resistance of respiratory system per } \mathrm{kg} \text { birth weight according to the single breath occlusion } \\
\text { technique. } 68\end{array}$} \\
\hline
\end{tabular}

\subsubsection{Statistical analyses}

A priori selection of potential predictors was based on clinical and physiological plausibility. After univariate analysis, the predictors were entered into multivariable logistic regression models as independent variables to assess their independent value in predicting prolonged ventilator need $(\geq 10$ days $)$ or need of postnatal steroid treatment. A combination of backward and forward stepwise independent selection was used. Final models consisted of independents with $p$-values less than 0.157 (Akaike's information criterion ${ }^{111}$ ), as was suggested by Steyerberg et al. ${ }^{226}$ for stepwise selection of independents in small data sets. Finally, selection variables were dichotomized.

Regression coefficients and corresponding odds ratios of both prediction models were presented after correction for overestimation according to the heuristic shrinkage method described by van Houwelingen and le Cessie. ${ }^{254}$ Subsequently, by calculating the area under the receiver operating characteristic curves (AUC) ${ }^{104}$ the overall discriminatory capacity of each model was obtained. AUC-values range from 0.5 (no discriminatory capacity) to 1.0 (perfect discrimination). The Hosmer and Lemeshow test was performed to test the 'goodness-of-fit' of each model. 
Table 3. Characteristics of dichotomous shrinked prediction models for each outcome variable.

\begin{tabular}{|c|c|c|c|}
\hline Independent & Regression coefficients & OR $(95 \% \mathrm{CI})^{\mathrm{a}}$ & Score \\
\hline \multicolumn{4}{|c|}{ Prolonged mechanical ventilation ( $\geq 10$ days): } \\
\hline Constant & -4.0 & & \\
\hline $\mathrm{R}_{\mathrm{rs}} / \mathrm{kg}(\geq 15 \mathrm{kPa} / \mathrm{L} \cdot \mathrm{s})^{\mathrm{b}}$ & 1.6 & $6.7(1.5-30.3)$ & 1 \\
\hline No. of ribs $(>16)^{c}$ & 1.9 & $9.7(1.6-58.0)$ & 1 \\
\hline Haziness (present) ${ }^{\mathrm{d}}$ & 2.2 & $13.8(1.2-161.6)$ & 1.5 \\
\hline \multicolumn{4}{|l|}{ Need of postnatal steroid therapy: } \\
\hline Constant & -2.8 & & \\
\hline Haziness (present) & 0.5 & $1.9(0.1-27.3)$ & 1 \\
\hline $\mathrm{R}_{\mathrm{rs}} / \mathrm{kg}(\geq 20 \mathrm{kPa} / \mathrm{L} \cdot \mathrm{s})$ & 1.3 & $4.7(1.0-21.6)$ & 2.5 \\
\hline Gestational age ( $<30$ weeks) & 1.7 & $7.7(0.8-76.4)$ & 3.5 \\
\hline \multicolumn{4}{|c|}{$\begin{array}{l}\text { a Odds ratio with } 95 \% \text { confidence interval. } \\
\text { b Resistance of respiratory system per kg birth weight according to the single-breath occlusion tech- } \\
\text { nique. }{ }^{68} \\
\text { c Mean lung volume score of two physicians according to the number of back ribs visible at the chest } \\
\text { radiograph in } 3 \text { categories: } 0=\text { less than } 14 \text { ribs; } 1=14 \text { to } 16 \text { ribs; } 2=\text { more than } 16 \text { ribs. } \\
\text { d Mean haziness score of two physicians according to } 4 \text { stages: } 0=\text { no haziness; } 1=\text { regionally localized } \\
\text { or around hilus; } 2=\text { regionally and around hilus; } 3=\text { total haziness. }\end{array}$} \\
\hline
\end{tabular}

To facilitate practical implementation, risk scores were developed and evaluated. Points were assigned to all predictors by dividing each coefficient by the smallest in the model and rounding it to halves. Total scores for individual infants were calculated by adding up the separate points for each predictor present. Subsequently, an observed risk of the outcome was obtained for the total score categories.

Our data were analysed with the Statistical Product and Service Solutions 10.0 for Windows (SPSS Inc, Chicago, IL, USA).

\subsection{Results}

Eighty-eight infants were initially included for the large longitudinal study. In 51 of 88 infants, chest radiographs of day 2 were available, but in three of them respiratory function measurements were unreliable according to ERS criteria. Hence, 48 premature infants were enrolled in the study. Patient characteristics are summarized in Table 1, showing wide ranges of GA, birth weight, 1- and 5-minute Apgar scores, umbilical cord $\mathrm{pH}$ and RDS classification. Twenty-eight infants received exogenous surfactant before 24 hours of age, i.e. before assessment of radiological or lung mechanical variables. Five infants received surfactant between 24 and 48 hours and relation to assessment of radiological or lung mechanical variables could not be detected. Three infants died while being mechanically ventilated. In one of them there 

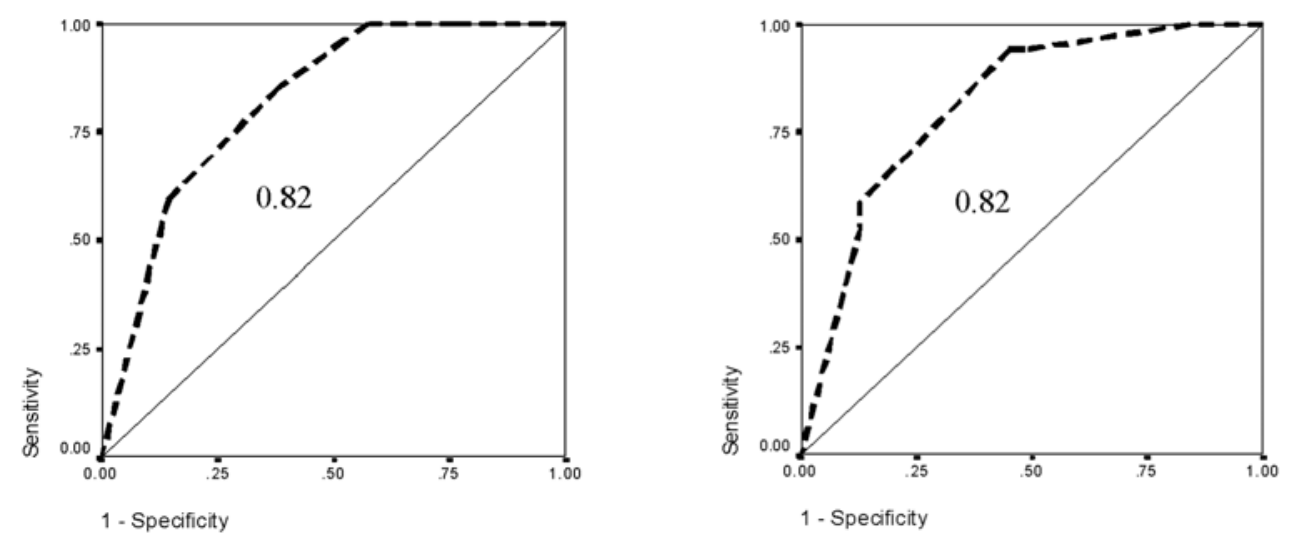

Figure 1 (left). ROC curve of the prediction model to predict prolonged mechanical ventilation $(\geq 10$ days) in preterm infants.

Figure 2 (right). ROC curve of the prediction model to predict postnatal steroid need in preterm infants.

was a discrepancy between the actual duration of mechanical ventilation and its severe respiratory condition, which was the reason it died. Therefore, it was allocated to the prolonged ventilation group, which eventually included infants who were mechanically ventilated for $\geq 10$ days or died because of respiratory insufficiency.

Results of univariate regression analysis are presented in Table 2. Following multivariate regression analysis, three out of seven a priori selected potential predictors were actually included in each logistic regression model (Table 3). The radiological items, haziness and number of posterior ribs bilaterally, significantly contributed to the prediction model for prolonged mechanical ventilation. GA and haziness were included in the prediction model for postnatal steroid therapy. $R_{\mathrm{rs}}$ expressed per kilogram birth weight $\left(\mathrm{R}_{\mathrm{rs}} / \mathrm{kg}\right)$ was additionally selected for both prediction models. With these models, the individual probability of outcome can be calculated for each patient fulfilling the inclusion criteria as follows: $1 / 1+\mathrm{e}^{\mathrm{LP}}$ where LP (linear predictor) is the sum of the constant and the regression coefficients for each variable present. For instance, the probability of prolonged mechanical ventilation of an infant with haziness on its chest radiograph, 14 posterior ribs visible, and a $R_{\mathrm{rs}} / \mathrm{kg}$ of $16 \mathrm{kPa} / \mathrm{L} \cdot \mathrm{s}$ per $\mathrm{kg}$ is $1 / 1+\mathrm{e}^{-(-4.0+2.2+1.6)}$, i.e. 45 per cent. Overall discriminatory capacity of each model was illustrated with ROC curves (Figures 1 and 2). The AUC was 0.82 for each outcome, representing a good discriminatory capacity 
Table 4. Relative number of infants with positive outcome for each score to predict prolonged mechanical ventilation ( $\geq 10$ days) and need of postnatal steroid therapy.

\begin{tabular}{lcc}
\hline Total score & $\begin{array}{c}\text { Probability of positive } \\
\text { outcome } \\
(\%)\end{array}$ & $\begin{array}{c}\text { Number of infants with positive outcome } \\
\text { / number of infants in score category }\end{array}$ \\
\hline $\begin{array}{l}\text { Prolonged mechanical ventilation }(\geq 10 \text { days): } \\
<2.5\end{array}$ & 9.1 & $1 / 11$ \\
2.5 & 55.6 & $10 / 18$ \\
$>2.5$ & 84.2 & $16 / 19$ \\
Total & 56.3 & $27 / 48$ \\
& & \\
Need of steroid therapy: & & $1 / 18$ \\
$<4.5$ & 5.6 & $6 / 16$ \\
4.5 & 37.5 & $10 / 14$ \\
$>4.5$ & 71.4 & $17 / 48$ \\
Total & 35.4 & \\
\hline
\end{tabular}

according to Weinstein et al. ${ }^{264}$ The Hosmer and Lemeshow test was not significant in neither model, indicating good fit.

Risk scores were perhaps more convenient but less precise. Points for each variable present were added up and, subsequently, the corresponding probability could be read from Table 4. Individual scores to predict prolonged mechanical ventilation and need of steroid treatment ranged from 0 to 3.5 and from 0 to 7.0 for the patients in our study, respectively, and were strongly related to the observed outcome probability, illustrated in Table 4. Using the same example as described earlier, the sum of the points would be 3.5 , i.e. $>2.5$, indicating a probability of approximately 84 per cent. Compared to the former calculation, resulting in a probability of 45 per cent, the probability based on the calculated risk score was much higher.

\subsection{Discussion}

In this study, reliable prediction models were constructed to select high-risk preterm infants for (1) prolonged mechanical ventilation ( $\geq 10$ days) and (2) need of systemic postnatal steroid therapy. Radiological haziness and hyperinflation, and respiratory system resistance were important predictors in the ventilation model. Gestational age, radiological haziness and respiratory system resistance were important in the steroid model. 
Both models can have relevant implications for clinical practice as well as for scientific research. Early reliable selection of high-risk infants of prolonged mechanical ventilation and postnatal corticosteroid therapy help to have one's priorities in clinical practice and to limit exposition to potentially adverse effects of new treatment strategies, when studying early administration of alternative antiinflammatory drugs. Although indications for postnatal steroid therapy might differ substantially between countries and medical centres, our steroid model can be widely used, when the indications for postnatal steroid administration, described in the methods section of this manuscript, are used as the outcome measures.

Our results were confirmed by other authors, who found decreased GA to predict adverse respiratory outcome. ${ }^{210,256,270}$ In contrast to these findings, acute lung inflammation is often less severe in extremely immature infants. One might think that in these infants long-term respiratory morbidity would be less severe as well. However, while RDS severity decreased during past decades accompanied by an increased survival of more immature infants, the incidence of CLD did not. ${ }^{58,125}$

Radiological risk scores were seldom studied this early after birth..$^{19,87,154}$ Chest radiographs later in respiratory course have the advantage of showing more radiological signs of advanced lung disease and may, therefore, become more sensitive in predicting respiratory outcome. Substantial interobserver variability of chest radiographs ${ }^{213}$ usually reduces their value in prediction models. ${ }^{270}$ In order to decrease interobserver variability two dichotomous radiological items were chosen which were easy to interpret.

Radiological appearance is also known to be affected by differences in ventilator modes. HFOV-conditions are usually determined by the inflation degree on chest radiographs (guidelines used at our NICU consisted of an inflation degree 8 to 8.5 ribs unilaterally during HFOV) and a constantly present high MAP during HFOV might decrease pulmonary oedema, and, thereby, haziness at the radiograph. In our study population, no significant differences were found between infants being conventionally ventilated and those ventilated by HFOV ( 77 per cent of the infants in both groups showed hyperinflation according to the criteria in Table $2(p=0.94) ; 81$ per cent of the conventionally ventilated infants showed haziness vs. 94 per cent of the infants receiving HFOV ( $p=0.21)$; data not shown). Administration of exogenous surfactant might affect radiological lung appearance as well. However, we found no such effect in our study population. Of the infants receiving surfactant within 24 hours after birth (28), 50 per cent had some degree of haziness at the chest radiograph and 50 per cent showed hyperinflation.

Lung mechanical characteristics have been studied before as potential predictors of the respiratory condition in preterm ventilated infants, but results are inconsistent. 19,46,70,89,137,160,256 Increased respiratory system resistance may both contribute to development of lung damage and be a manifestation of CLD itself. Airway damage secondary to endotracheal intubation, positive pressure, and/or high oxygen 
concentrations may cause increased respiratory system resistance, as may increased pulmonary blood flow or left heart failure secondary to a patent ductus arteriosus (PDA) causing peribronchial oedema. ${ }^{89}$ However, we did not find a significant difference in PDA prevalence (confirmed by ultrasound performed by a paediatric cardiologist) between infants with high and infants with low $R_{\mathrm{rs}}(29 \%$ vs. $27 \%$, respectively; $p=0.88$, when using a cut-off value of $20 \mathrm{kPa} / \mathrm{L} \cdot \mathrm{s}$; and $33 \%$ vs. $19 \%$, respectively; $p=0.27$, when using a cut-off value of $15 \mathrm{kPa} / \mathrm{L} \cdot \mathrm{s})$.

Although $C_{\mathrm{rs}}$ decreases in the presence of respiratory disease in preterm infants, we did not find this variable to be an important predictor of clinical respiratory course. Previous studies performed before the surfactant era were also inconsistent. 19,46,62,70,89,91,137 219,256 Although administration of exogenous surfactant is known to immediately affect $C_{\mathrm{rs}},{ }^{45}$ we did not find significant differences in surfactant administration between infants with high and infants with lower $C_{\mathrm{rs}}$ values (data not shown).

There are some remarks concerning the study design. The statistical power of the study was limited as a consequence of the small study population. Therefore, the number of a priori selected predictors was limited, increasing the risk of missing valuable predictors, for example, ventilator conditions, which have been studied as potential predictors of respiratory course before. ${ }^{91,137,214,25,270}$ Since in small data sets regression coefficients tend to overestimate, we used the heuristic shrinkage method to correct for this effect. However, external prospective validation in a comparable group of infants is still recommended before widespread use of the prediction models can be encouraged. Although in the majority of studies a pre-selection of infants was made based on GA $<30$ or $<32$ weeks or birth weights of $<1500$ or $<1000$ grams ${ }^{19,95,210,214,270}$ in order to optimize sensitivity of the models, we preferred to enroll all preterm infants of $<37$ weeks gestation, as did Toce et al. ${ }^{242}$ and Lischka et al., ${ }^{154}$ to include different pathologic aspects in both extremely immature infants and less premature infants. Switching ventilator conditions from HFOV to SIMV to make lung function testing possible might have caused a selection bias, excluding most severely ill patients not capable to undergo this switch without problems, or a measurement bias when reactive bronchospasms occurred after this switch. Since in only one infant of our study this switch was needed, we assumed this effect to be trivial.

Both prolonged mechanical ventilation and need for postnatal steroid therapy are known to be strongly associated with CLD development. However, the main advantage of our models is the possibility to make separate selections. Early selection of high-risk infants for prolonged mechanical ventilation may have important consequences for clinical practice in having one's priorities concerning preventive strategies. Early selection of infants at risk of late steroid therapy may increase research possibilities for new treatment strategies by decreasing the risk of unnecessary exposure. The Cochrane Review ${ }^{101}$ about early postnatal steroid therapy 
( $<96$ hours after birth) implicated an urgent need of further studies investigating different doses and schedules of steroid treatment, effects of inhaled steroids and potentially safer steroids.

In summary, reliable prediction of prolonged mechanical ventilation $(\geq 10$ days $)$ and need of postnatal steroid therapy in preterm infants can be performed before 48 hours after birth with clinical, radiological and lung mechanical variables. Prospective external validation of these prediction models is recommended before widespread application can be encouraged. Eventually, early selection will increase the potential to improve preventive strategies as well as further research concerning new interventions in preterm ventilated infants. 


\section{CHAPTER 6}

\section{Respiratory outcome in preterm ventilated infants: importance of early respiratory system resistance}

Yvonne Snepvangers

Peter de Winter

Huibert Burger

Hens Brouwers

Kors van der Ent 



\subsection{Abstract}

Because severity of acute respiratory distress (RDS) has been decreasing, aetiology of long-term respiratory problems may have changed in preterm infants. We investigated the causal relationships between early neonatal respiratory resistance $\left(R_{\mathrm{rs}}\right)$ and compliance $\left(C_{\mathrm{rs}}\right)$ and development of respiratory morbidity and lung function abnormalities during the first year of life in ventilated preterm infants.

In 70 infants of less than 37 weeks gestation, mechanically ventilated within 24 hours after birth, $R_{\mathrm{rs}}$ and $C_{\mathrm{rs}}$ were assessed daily during the first 3 days of life and medians were calculated subsequently $\left(R_{\mathrm{rs}, \text { neo }}\right.$ and $\left.C_{\mathrm{rs}, \text { neo }}\right) . R_{\mathrm{rs}}$ and $C_{\mathrm{rs}}$ were reassessed 1 year later in 57 of 70 infants $\left(R_{\mathrm{rs}, 1 \text { year }}\right.$ and $\left.C_{\mathrm{rs}, 1 \text { year }}\right)$.

After correction for endotracheal tube size, increased $R_{\mathrm{rs}, \text { neo }}$ was significantly related to respiratory morbidity during the first year of life (odds ratio, 1.20; 95\% confidence interval (CI), 1.04 to $1.38 ; p=0.013$ ), increased $R_{\mathrm{rs}, 1 \text { year }}$ (multiplicative $\beta$ per $\mathrm{kPa} / \mathrm{L} \cdot \mathrm{s}, 1.017 ; 95 \% \mathrm{CI}, 1.000$ to $1.034 ; p=0.045$ ), and decreased $C_{\mathrm{rs}, 1 \text { year }}$ (multiplicative $\beta$ per kPa $/ \mathrm{L} \cdot \mathrm{s}=0.986 ; 95 \% \mathrm{CI}, 0.974$ to $0.998 ; p=0.023$ ). Associations were not adversely affected by degree of prematurity, neonatal disease and treatment. $C_{\mathrm{rs}, \text { neo }}$ did not show any significant associations.

In the current surfactant era, increased $R_{\mathrm{rs}, \text { neo, }}$ and not $C_{\mathrm{r}, \text { neoo, }}$ was associated with poor respiratory outcome during the first year of life in preterm ventilated infants. Neonatal disease and treatment seemed to be of minor importance compared to inborn properties of the respiratory system.

\subsection{Introduction}

The introduction of prenatal corticosteroids and postnatal exogenous surfactant treatment in perinatal care, probably have played a major role in the observed trend of decreasing severity of respiratory distress syndrome (RDS) during the past decades. ${ }^{32}$ Nevertheless, the risk of developing chronic lung disease of the neonate (CLD) has remained high and may even have increased..$^{58}$

Respiratory system resistance $\left(R_{\mathrm{rs}}\right)$ and compliance $\left(C_{\mathrm{rs}}\right)$ have been extensively studied in preterm infants. Results relating these parameters to respiratory outcome are conflicting, particularly with regard to the development of CLD. 19,70,89,91,137,160,219,256 Moreover, most of these studies were performed before the use of prenatal corticosteroids and the introduction of exogenous surfactant therapy. As a consequence of these recent developments in perinatal care, the survival of more immature infants has increased. Acute respiratory disease is often less severe and respiratory outcome seems to be mainly determined by an arrest of lung development in these infants. ${ }^{125}$ Antenatal corticosteroid treatment may also cause impaired postnatal lung growth. ${ }^{108,247}$ So, contemporary associations between early postnatal $R_{\mathrm{rs}}$ 
and $C_{\mathrm{rs}}$ and long-term respiratory outcome may have changed compared to the presurfactant era.

The purpose of this study was to investigate whether neonatal respiratory mechanics play a role in the aetiology of respiratory morbidity and lung function abnormalities during infancy in preterm ventilated infants and whether other relevant neonatal factors, like degree of prematurity, ventilator conditions, respiratory disease severity and therapeutic measures, affected these relationships.

\subsection{Subjects and methods}

Preterm infants of less than 37 weeks of gestation, who were admitted to our neonatal intensive care unit between 28 July 1999 and 31 August 2000 and who were mechanically ventilated within 24 hours after birth, were studied prospectively. Infants with congenital anomalies were excluded from the study. The study was approved by the Medical Ethics Review Committee of our centre and parental informed consent was obtained.

Mechanical ventilation was started as a consequence of respiratory distress, when, despite supplemental oxygen and/or continuous positive airway pressure, the inspiratory oxygen fraction $\left(\mathrm{FiO}_{2}\right)$ remained above 0.7 , the partial arterial carbon dioxide pressure exceeded $55 \mathrm{~mm} \mathrm{Hg}$, acidosis persisted with $\mathrm{pH}$ decreasing below 7.25 , or when serious apnoeas existed. Ventilator setting was adjusted to keep the arterial oxygen saturation of haemoglobin between 90-95 per cent and the partial arterial carbon dioxide pressure between $40-45 \mathrm{~mm} \mathrm{Hg}$. For mechanical ventilation, one of the following infant respirators was used: Stephanie (Stephan, Gackenbach, Germany), Bear BP2001 (Bear, Palm Springs, CA, USA), and Bourns BP200 (Bourns, Inc., Riverside, CA, USA).

RDS was diagnosed when clinical symptoms of tachypnoea $(>60 / \mathrm{min})$, retractions, expiratory grunting and cyanosis were present in combination with radiological signs described by Giedion. ${ }^{86}$ Exogenous surfactant (natural bovine surfactant: Alvofact, Boehringer, Ingelheim, Germany) was administered at a dose of $50 \mathrm{mg} / \mathrm{kg}$ using the following limits of peak inspiratory pressure (PIP) and $\mathrm{FiO}_{2}$ : PIP $<20 \mathrm{cmH}_{2} \mathrm{O}$ and $\mathrm{FiO}_{2}>0.6$, or PIP $\geq 20 \mathrm{cmH}_{2} \mathrm{O}$ and $\mathrm{FiO}_{2}>0.45$. When PIP exceeded $25 \mathrm{cmH}_{2} \mathrm{O}$, conventional ventilation was switched to high frequency oscillatory ventilation (HFOV).

Measurements of passive respiratory system mechanics were performed daily during the first 3 days of life in intubated infants. No extra sedation of the infants was required. Sometimes measurements could not be performed, because of clinical instability of the patient, unavailability of qualified personnel, i.e. two of the contributing authors (Y.S. and P.d.W.), or because patients were extubated before the actual time of measurement. To optimize the total number of measurement results, 
the median passive respiratory system resistance $\left(R_{\mathrm{rs}, \mathrm{neo}}\right)$ and compliance $\left(C_{\mathrm{r}, \mathrm{nneo}}\right)$ of the first 3 days of life were calculated. $R_{\mathrm{rs}}$ and $C_{\mathrm{rs}}$ were assessed according to the single breath occlusion technique (SBT) described by Le Souef. ${ }^{68,150}$ A heated Lilly-type pneumotachometer $(8410 \mathrm{~A}$, linear range $0-10 \mathrm{~L} / \mathrm{min}$, dead space $1.3 \mathrm{ml}$, resistance $0.94 \mathrm{kPa} / \mathrm{L} \cdot \mathrm{s}$ at $5 \mathrm{~L} / \mathrm{min}$; Hans Rudolph Inc., Kansas City, MO, USA) was placed between endotracheal tube and ventilator tubes. A pressure transducer (Honeywell, type 163PC01D75, Morristown, NJ, USA) and an IBM compatible personal computer with a Pentium processor running the software package Respiratory Analysis System Program (Version 007.17, Physio Logic, High Clere, Great Britain) were used to record signals at a sampling rate of $125 \mathrm{~Hz}$ using a 12 bit analogue-to-digital converter (LabMaster DMA, Scientific Solutions, Mentor, OH, USA). Mouth pressure and flow signals were obtained after calibration before each single measurement. For flow calibration, a calibrated rotameter (Air 0-15 L/min, Medec, Aalst, Belgium) was used followed by a volume calibration using a precision syringe of $50-\mathrm{ml}$ containing the actual gas mixture. For pressure calibration, a pressure transducer tester (Veri-Cal 650900, Utah Medical Products Inc., Midvale, UT, USA) was used. Leaks in the system or around the endotracheal tube were easily detected as mouth pressure decreased gradually during occlusion. With gentle manual compression of the trachea present leaks were minimized. Measurements with persisting tube leaks causing a decrease in occlusion pressure of $0.02 \mathrm{kPa}$ or more were excluded from further analysis. 45,220 During occlusion, activity of inspiratory muscles is inhibited via stimulation of the airway receptors inducing the Hering-Breuer reflex. Analyses were performed according to the actual European Respiratory Society (ERS) ${ }^{61}$ Breath analysis was considered unreliable, when no muscle relaxation was achieved. Muscle relaxation was assumed to be present when (1) during a short occlusion period of less than 0.5 seconds mouth pressure did reach a plateau for at least 0.1 seconds during which pressure changes showed a standard deviation (SD) of $0.01 \mathrm{kPa}$ or less, and (2) the expiration part of the flow volume curve was linear for at least 50 per cent of the volume to be expired. As a criterion for linearity, a correlation coefficient of 0.98 or more after exclusion of the final 5 per cent of the expired volume had to be reached. At least five occluded breaths meeting these criteria were used to calculate a mean value for both respiratory mechanics for each single measurement.

At the age of one, $R_{\mathrm{rs}, 1 \text { year }}$ and $C_{\mathrm{rs}, 1 \text { year }}$ were determined during spontaneous breathing using the same technique (SBT). At this age, infants were sedated with 50 $100-\mathrm{mg} / \mathrm{kg}$ chloral hydrate and measurements were performed in supine position with the head end elevated at approximately 30 degrees relative to horizontal. A heated Lilly-type pneumotachometer $(3500 \mathrm{~B}$, linear range $0-35 \mathrm{~L} / \mathrm{min}$, dead space $8.74 \mathrm{ml}$, resistance $0.32 \mathrm{kPa} / \mathrm{L} \cdot \mathrm{s}$ at $17.5 \mathrm{~L} / \mathrm{min}$; Hans Rudolph Inc., Kansas City, MO, USA) was attached to a facemask (Laerdal Infant Mask No.1, Laerdal BeNelux BV, Valkenswaard, The Netherlands; dead space $10 \mathrm{ml}$ ) covered with therapeutic putty (Magic Putty, medium, Oldelft Benelux BV, Delft, The Netherlands) to prevent air leakage. 


\section{Respiratory outcome one year after preterm birth}

To evaluate internal validity of the tests, the intrasubject variability of both $R_{\mathrm{rs}}$ and $C_{\mathrm{rs}}$ in mechanically ventilated infants was studied.228 Coefficients of variance of $R_{\mathrm{rs}}$ and $C_{\mathrm{rs}}$ were determined within subjects within each occasion and within subjects between up to three occasions that were used to calculate median $R_{\mathrm{rs}}$ and $C_{\mathrm{rs}}$ of the first 3 days of life.

Clinical data, i.e. gestational age, birth weight, severity of RDS according to Giedion, ${ }^{86}$ duration of mechanical ventilation, mean PIP during ventilation, number of days requiring a $\mathrm{FiO}_{2}$ of more than 0.4 , and oxygen dependency at 36 weeks gestational age, was derived from clinical charts. To achieve an indication of respiratory morbidity after hospital discharge, data about inhaled corticosteroid or bronchodilator therapy and hospital readmission for respiratory problems were obtained via questionnaires completed by the parents at the end of the first year. The questions in these questionnaires were similar to the key questions in the questionnaire used by Kuehni et al. ${ }^{144}$ in children between 0 and 5 years of age. Questions included (translated from Dutch):

- During the past 12 months, has your child taken medicine for wheezing or coughing?

- If so, which medication was taken?

- During the past 12 months, has your child been admitted to hospital for respiratory problems?

If inhaled corticosteroids or bronchodilators were used, or rehospitalization occurred during the first year of life respiratory morbidity was judged as present.

\subsubsection{Data analysis}

Characteristics of the patients who were lost to follow-up were compared to the characteristics of the patients who returned for follow-up measurements by independent Student's $t$-tests, when data were normally distributed and variances were equal. Otherwise, Mann-Whitney $U$-tests were used. For comparison of frequencies of dichotomous variables between groups, Chi-square analyses were performed.

As a first evaluation of data, associations between early neonatal clinical and functional data (as independent variables) and respiratory morbidity, $R_{\mathrm{rs}, 1 \text { year, }}$, and $C_{\mathrm{rs}, 1 \text { year }}$ (as dependent variables) were studied by univariate regression analyses. Since endotracheal tube size substantially affects $R_{\mathrm{rs}}$ measurements during mechanical ventilation, ${ }^{152,164} R_{\mathrm{rs}, \text { neo }}$ was subsequently corrected for this effect by including tube diameter in all regression models. In these cases, we will refer to $R_{\mathrm{rs}, \text { neoc. }}$. Respiratory morbidity during the first year of life was expressed dichotomously ( 1 for present and 0 for absent) and logistic regression analyses were used regarding this outcome measure. $R_{\mathrm{rs}, 1 \text { year }}$ and $C_{\mathrm{rs}, 1 \text { year }}$ were transformed by taking the natural logarithm, because 
of their deviation from the normal distribution. Since results of regression analyses are presented after back transformation, regression coefficients and their $95 \%$ confidence intervals (CI) should be interpreted as multiplicative instead of additive.

Subsequently, aetiological models were constructed and their independency from potentially confounding factors was studied (i.e. clinical data, such as gestational age, birth weight, severity of RDS according to Giedion, ${ }^{86}$ duration of mechanical ventilation, mean PIP during ventilation, number of days requiring a $\mathrm{FiO}_{2}$ of more than 0.4 , and oxygen dependency at 36 weeks gestational age). Only factors affecting the initial regression coefficients of the respiratory mechanics by more than 10 per cent were included in the models and discussed subsequently.

Our data were analysed with the Statistical Product and Service Solutions 10.0 for Windows (SPSS Inc, Chicago, IL, USA). For all analyses, a $p$-value of $<0.05$ was considered statistically significant.

\subsection{Results}

Initially, 88 infants were randomly recruited. Eleven infants died during the neonatal period leaving 77 infants for follow-up at the age of one. Six infants died of respiratory insufficiency, four due to neurological problems and one due to circulatory problems. $R_{\mathrm{rs}, \text { neo }}$ and $C_{\mathrm{rs} \text {,neo }}$ results in seven infants were considered unreliable according to the ERS criteria and, therefore, excluded from further analysis. Eventually, data in 70 infants were analysed. Patient characteristics of this group are listed in Table 1.

Information about respiratory morbidity during the first year of life was complete for $66 / 70(94 \%)$ infants. In $57 / 70(81 \%)$ infants, respiratory function measurements were performed at the age of one. Parents of 10 infants decided not to return for follow-up respiratory function measurements for various personal reasons, in two cases no reason was given, and one infant was tested 3 months late and therefore excluded for further analysis. The 13/70 (19\%) infants, in whom follow-up data were missing, did not substantially differ from the $57 / 70$ (81\%) infants, in whom these data were available, with respect to gestational age (median (range), 29.9 (26.6-33.7) vs. 29.4 (25.3-36.3) weeks, respectively; $p=0.794)$, birth weight $(1536$ (875-1860) vs. 1240 (630-3020) $\mathrm{g} ; p=0.572$ ), sex (54\% vs. 56\% male; $p=0.881$ ), maximum PIP (23 (14-40) vs. $\left.22(15-35) \mathrm{cmH}_{2} \mathrm{O} ; p=0.387\right)$, Giedion score (3 (0-4) vs. $\left.3(0-4) ; p=0.268\right)$, and oxygen dependency at 36 weeks post-conceptional age ( $0 \%$ vs. $7 \% ; p=0.325)$. However, groups did differ with respect to duration of mechanical ventilation (5 (115) vs. 11 (1-67) days; $p=0.014)$ and number of days requiring $\mathrm{FiO}_{2}$ of $>0.4(0(0-1)$ vs. $1(0-48)$ days; $p=0.008)$. $R_{\mathrm{rs}, \text { neo }}$ and $C_{\mathrm{rs} \text {,neo }}$ were comparable between both groups (14.9 (9.8-22.1) vs. $15.3(8.8-30.4) \mathrm{kPa} / \mathrm{L} \cdot \mathrm{s} ; ~ p=0.381$, and $5.9(3.6-12.1)$ vs. $5.2(2.3-$ 
22.2) $\mathrm{ml} / \mathrm{kPa} ; p=0.962$, respectively), as was the occurrence of respiratory morbidity, i.e. inhalation therapy or hospital readmission, during the first year of life $(50 \%$ vs. $41 \%$; $p=0.557)$.

Table 1. Patient characteristics.

\begin{tabular}{|c|c|c|}
\hline & & $\begin{array}{c}\text { Median (range) / } \\
\text { Number }(\%)\end{array}$ \\
\hline Neonatal period & $n=70)$ & \\
\hline Gestational age (weeks) & & $29.5(25.3-36.3)$ \\
\hline Birth weight (grams) & & $1277(630-3020)$ \\
\hline Sex (male) & & $39(56 \%)$ \\
\hline Prenatal exposure to tobacco smoke & & $16(23 \%)$ \\
\hline Prenatal corticosteroids administration ( $\geq 24 \mathrm{~h}$ antenatally) & & $45(64 \%)$ \\
\hline Radiological RDS severitya & & $3(0-4)$ \\
\hline Duration of mechanical ventilation (days) & & $9(1-67)$ \\
\hline Maximum peak inspiratory pressure $\left(\mathrm{cm} \mathrm{H}_{2} \mathrm{O}\right)$ & & $22(14-40)$ \\
\hline Number of days with $\mathrm{FiO}_{2}>0.4$ & & $1(0-48)$ \\
\hline Surfactant administration & & $46(66 \%)$ \\
\hline Hour of life of surfactant instillation & & $9(1-52)$ \\
\hline Diagnosis of a patent ductus arteriosus $(\mathrm{PDA})^{\mathrm{b}}$ & & $27(39 \%)$ \\
\hline Day of PDA diagnosis & & $2(1-9)$ \\
\hline \multicolumn{3}{|l|}{ Size of endotracheal tube (internal diameter): } \\
\hline $2.5 \mathrm{~mm}$ & & $35(50 \%)$ \\
\hline $3.0 \mathrm{~mm}$ & & $32(46 \%)$ \\
\hline $3.5 \mathrm{~mm}$ & & $2(3 \%)$ \\
\hline Switched from 2.5 to $3.0 \mathrm{~mm}$ at second day of life & & $1(1 \%)$ \\
\hline$R_{\mathrm{rs}, \mathrm{neo}}(\mathrm{kPa} / \mathrm{L} \cdot \mathrm{s})$ & & $15.1(8.8-30.4)$ \\
\hline$C_{\mathrm{rs}, \text { пеo }}(\mathrm{ml} / \mathrm{kPa})$ & & $5.4(2.3-22.2)$ \\
\hline Oxygen dependency at 36 weeks & & $4(6 \%)$ \\
\hline Postnatal corticosteroid administration & & $23(33 \%)$ \\
\hline \multicolumn{3}{|l|}{ Outcome during the first year of life } \\
\hline Inhalation therapy with bronchodilators and/or steroids & $(n=66)$ & $27(41 \%)$ \\
\hline Rehospitalization for respiratory problems & & $9(14 \%)$ \\
\hline Respiratory morbidityc ${ }^{1}$ & & $28(42 \%)$ \\
\hline$R_{\mathrm{rs}, 1 \text { year }}(\mathrm{kPa} / \mathrm{L} \cdot \mathrm{s})$ & $(n=57)$ & $4.6(2.7-8.3)$ \\
\hline$C_{\mathrm{rs}, 1 \text { year }}(\mathrm{ml} / \mathrm{kPa})$ & & $99.2(59.6-169.3)$ \\
\hline Age at follow-up measurement corrected for gestation (days) & & $303(270-437)$ \\
\hline Weight at follow-up measurement $(\mathrm{kg})$ & & $8.8(6.6-12.0)$ \\
\hline Height at follow-up measurement (cm) & & $72.3(65.0-80.0)$ \\
\hline
\end{tabular}




\subsubsection{Respiratory function measurements during mechanical ventilation}

In the initial study population of 70 infants, 55 assessments of $C_{\mathrm{rs}}$ were obtained at day 1, 48 at day 2, and 45 at day 3 . Since in a few of these occasions non-linear expiratory flow patterns precluded reliable $R_{\mathrm{rs}}$ assessment, $53 R_{\mathrm{rs}}$ assessments were obtained at day 1, 45 at day 2 and 44 at day 3 . The median number of occluded breaths (range), on which results of each single measurement were based on, was 6 (312). In nine measurements the amount of five occluded breaths was not reached and results were based on four reliable occluded breaths in five measurements and three reliable occluded breaths in four measurements. Of all neonatal respiratory function measurements, the median $\mathrm{CV}$ (range) within subjects within measurements of $C_{\mathrm{rs}}$ was 7.7 (1.5-38.7) and of $R_{\mathrm{rs}}$ was $6.4(1.8-51.3)$. CV values within subjects between measurements of the first 3 days of life were calculated in 68 per cent of the $C_{\mathrm{rs}}$ measurements and in 64 per cent of the $R_{\mathrm{rs}}$ measurements where respiratory function measures were based on more than one measurement. The median CV (range) between $C_{\mathrm{rs}}$ measurements was $24.9(0.8$ to 60.0$)$ and between $R_{\mathrm{rs}}$ measurements 15.6 (4.2 to 62.1). The median CV (range) between $R_{\mathrm{rs}}$ measurements was 15.6 (4.2-62.1) and between $C_{\mathrm{rs}}$ measurements $24.9(0.8-60.0)$.

\subsubsection{Relationships between early postnatal factors and respiratory outcome during infancy}

Univariate regression analyses revealed no significant associations between early neonatal clinical data (gestational age, birth weight, radiological RDS severity according to Giedion, duration of mechanical ventilation, maximal PIP, number of days requiring a $\mathrm{FiO}_{2}$ of more than 0.4, and oxygen dependency at 36 weeks gestational age) and respiratory morbidity during the first year of life and $R_{\mathrm{rs}, 1 \text { year. }}$. We did find, however, significant associations between gestational age, birth weight, and duration of mechanical ventilation and $C_{\mathrm{rs}, 1 \text { year }}($ Table 2).

Increased $\mathrm{R}_{\mathrm{rs}, \text { neo }}$ was associated with respiratory morbidity during the first year of life (odds ratio (OR), 1.20; 95\% confidence interval (CI), 1.04 to $1.38 ; p=0.013$ ). The association between the actually measured values of $R_{\mathrm{rs}, \text { neo }}$ and respiratory morbidity is presented in Figure 1. Although independent of gestational age, birth weight, radiological RDS severity according to Giedion, ${ }^{86}$ duration of mechanical ventilation, number of days requiring a $\mathrm{FiO}_{2}$ of more than 0.4 , and oxygen dependency at 36 weeks gestational age, the association between $R_{\mathrm{rs}, \text { neoC }}$ and respiratory morbidity was affected by the maximal PIP used during mechanical ventilation. After including maximal PIP as independent factor into the regression model, the initial relation between $R_{\mathrm{rs}, \mathrm{neo}}$ and respiratory morbidity became stronger and more significant (odds ratio, 1.23; 95\% CI, 1.05 to 1.43; $p=0.009$ ), but maximal PIP itself was not associated with respiratory morbidity (odds ratio, $0.90 ; 95 \% \mathrm{CI}, 0.78$ 
Table 2. Results of univariate regression analyses with $C_{\mathrm{rs}, 1 \text { year }}$ as a dependent variable.

\begin{tabular}{|c|c|c|c|}
\hline Early postnatal factor & $\begin{array}{l}\text { Multipli- } \\
\text { cative } \beta \\
\text { per unit }\end{array}$ & $\begin{array}{l}95 \% \\
\text { confidence } \\
\text { interval of } \beta\end{array}$ & $p$-value \\
\hline Gestational age (weeks) & 1.035 & $1.013-1.057$ & 0.002 \\
\hline Birth weight (grams) & 1.0002 & $1.000-1.000$ & 0.001 \\
\hline Radiological RDS severity ${ }^{a}$ & 1.005 & $0.954-1.055$ & 0.851 \\
\hline Duration of mechanical ventilation (days) & 0.996 & $0.992-0.999$ & 0.018 \\
\hline Maximum peak inspiratory pressure $\left(\mathrm{cm} \mathrm{H} \mathrm{H}_{2} \mathrm{O}\right)$ & 0.995 & $0.979-1.011$ & 0.510 \\
\hline Number of days with $\mathrm{FiO}_{2}>0.4$ & 0.993 & $0.986-1.000$ & 0.062 \\
\hline Oxygen dependency at 36 weeks post-conceptional age & 1.229 & $0.991-1.466$ & 0.059 \\
\hline $\boldsymbol{R}_{\mathrm{rs}, \text { neoC }}(\mathrm{kPa} / \mathrm{L} \cdot \mathrm{s})$ & 0.986 & $0.974-0.998$ & 0.023 \\
\hline$C_{\mathrm{rs}, \text { neo }}(\mathrm{ml} / \mathrm{kPa})$ & 1.015 & $1.000-1.030$ & 0.049 \\
\hline
\end{tabular}

${ }^{a}$ Radiological score for severity of respiratory distress syndrome according to Giedion (0-4). ${ }^{86}$

to $1.04 ; p=0.149)$. Secondly, increased $R_{\mathrm{rs}, \text { neoC }}$ was related to increased $R_{\mathrm{rs}, \text { 1year }}$ (multiplicative $\beta$ per $\mathrm{kPa} / \mathrm{L} \cdot \mathrm{s}, 1.017 ; 95 \% \mathrm{CI}, 1.000$ to $1.034 ; p=0.045$ ) and this association was independent of all potentially confounding factors. Thirdly, $R_{\mathrm{rs}, \mathrm{neoC}}$ was negatively associated with $C_{\mathrm{rs}, 1 \text { year }}$ (multiplicative $\beta$ per kPa/L·s $=0.986 ; 95 \% \mathrm{CI}$, 0.974 to $0.998 ; p=0.023$ ). Of all potential confounding factors, the number of days requiring a $\mathrm{FiO}_{2}$ of more than 0.4 substantially affected this association. After including this variable into the regression model, the initial association between $R_{\mathrm{rs}, \mathrm{neoC}}$ and $C_{\mathrm{rs}, 1 \text { year }}$ became more significant (multiplicative $\beta$ per kPa $/ \mathrm{L} \cdot \mathrm{s}=0.984 ; 95 \% \mathrm{CI}$, 0.972 to $0.995 ; p=0.007$ ) and the number of days requiring a $\mathrm{FiO}_{2}$ of more than 0.4 itself was negatively associated with $C_{\mathrm{rs}, 1 \text { year }}$ (multiplicative $\beta$ per day $=0.992 ; 95 \% \mathrm{CI}$, 0.986 to $0.999 ; p=0.019)$.

$C_{\mathrm{rs}, \text { neo }}$ was positively associated with $C_{\mathrm{rs}, 1 \text { year }}$ (multiplicative $\beta$ per $\mathrm{ml} / \mathrm{kPa}, 1.015$; $95 \%$ CI, 1.000 to $1.030 ; p=0.049)$. However, after correction for birth weight this association lost statistical significance (multiplicative $\beta$ per $\mathrm{ml} / \mathrm{kPa}, 1.001 ; 95 \% \mathrm{CI}$, 0.984 to $1.018 ; p=0.885$ ) and birth weight itself was significantly associated with $C_{\mathrm{rs}, 1 \text { year }}$ (multiplicative $\beta$ per kg, 1.181; 95\% CI, 1.052 to $1.310 ; p=0.007$ ). $C_{\mathrm{rs}, \text { neo }}$ was neither associated with $R_{\mathrm{rs}, 1 \text { year }}$ (multiplicative $\beta$ per $\mathrm{ml} / \mathrm{kPa}, 0.998 ; 95 \% \mathrm{CI}, 0.990$ to $1.007 ; p=0.653)$ nor with respiratory morbidity (OR, 0.96 ; $95 \% \mathrm{CI}, 0.84$ to 1.09 ; $p=0.495)$. 


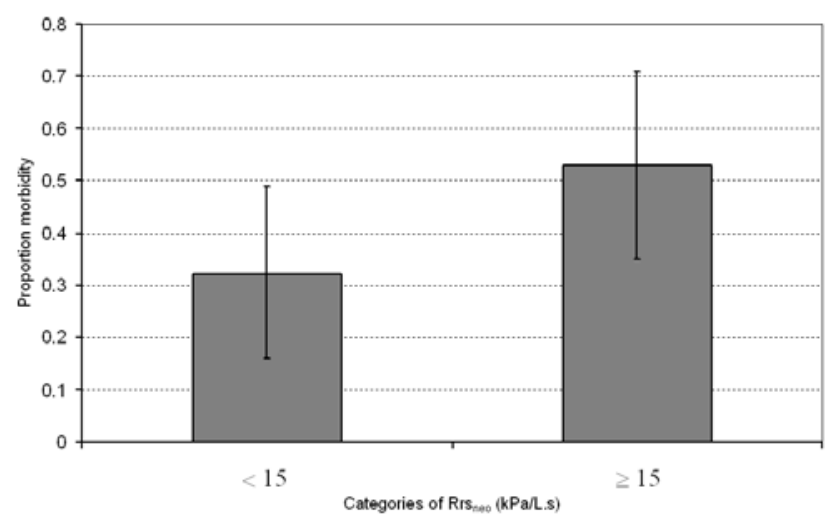

Figure 1. Mean proportions of infants with respiratory morbidity (i.e. inhaled corticosteroid or bronchodilator therapy, or rehospitalization) during the first year of life for two categories of

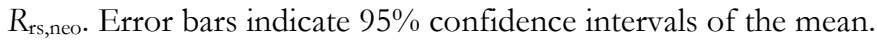

\subsection{Discussion}

We found that, in the current surfactant era, early neonatal $R_{\mathrm{rs}}\left(R_{\mathrm{rs}, n e o c}\right)$, and not $C_{\mathrm{rs}}\left(C_{\mathrm{rs}, \mathrm{neo}}\right)$, was associated with respiratory outcome during infancy. Increased $R_{\mathrm{rs}, \text { neoC }}$ was associated with respiratory morbidity during the first year of life, increased $R_{\mathrm{rs}}$ $\left(R_{\mathrm{rs}, 1 \text { year }}\right)$ and decreased $C_{\mathrm{rs}}\left(C_{\mathrm{rs}, 1 \text { year }}\right)$ at 1 year of age. These causal relationships were not affected by factors indicating degree of prematurity and neonatal disease. The relationship between $R_{\mathrm{rs}, \text { neoC }}$ and respiratory morbidity was strengthened by inclusion of maximal PIP required during mechanical ventilation in the neonatal period into the regression model. The relationship between $R_{\mathrm{rs}, \mathrm{neoC}}$ and $C_{\mathrm{rs}, 1 \text { year }}$ was strengthened by the number of days requiring a $\mathrm{FiO}_{2}$ of more than 0.4. Early postnatal $C_{\mathrm{rs}, \text { neo }}$ was not associated with respiratory outcome during the first year of life. Degree of prematurity and duration of mechanical ventilation were also associated with $C_{\mathrm{rs}, 1 \text { year. }}$.

$R_{\mathrm{rs}, \mathrm{neoC}}$ was the most consistent factor related to respiratory outcome during the first year of life in ventilated preterm infants. $R_{\mathrm{rs}}$ represents the sum of the resistive properties of chest wall, airways and, especially in immature infants, the lung parenchyma ${ }^{199}$ Acute respiratory disease in preterm infants is dominated by parenchymal changes, like surfactant deficiency, alveolar hyaline membrane deposition, interstitial oedema, and pulmonary interstitial emphysema. Because treatment facilities for acute respiratory disease have improved dramatically, early postnatal $R_{\mathrm{rs}}$ increasingly reflects inborn properties of the respiratory system, ${ }^{68}$ 
depending on gestational age, intra-uterine lung development, and familial predisposition. It has been suggested before that both interruption of antenatal lung development by preterm birth ${ }^{58,125}$ and intra-uterine growth retardation ${ }^{59}$ are important indicators of respiratory outcome in preterm infants. In a study of very low birth weight infants, Chan et al. ${ }^{30}$ found that conductance (the reciprocal of resistance) in the first year of life was not associated with neonatal oxygen score and duration of mechanical ventilation. Lui et al., ${ }^{160}$ who found that $R_{\mathrm{rs}}$, measured during the first week of life in 46 infants with birth weights of $<1000 \mathrm{~g}$, was related to respiratory outcome, while $C_{\mathrm{rs}}$ was not. They also found that $R_{\mathrm{rs}}$ was a better predictor of CLD development than RDS and surfactant use. These findings support our findings and argue that inborn properties of the respiratory system more strongly affect respiratory outcome than acute neonatal lung injury by RDS or ventilator conditions.

Both gestational age and birth weight, as markers of intra-uterine body growth, did not affect the relationships between $R_{\mathrm{rs}, \text { neoC }}$ and respiratory outcome. Intra-uterine lung growth may be completely incorporated by $R_{\mathrm{rs}, \text { neo }}$, and may therefore not be a real confounder. We found that inspiratory pressures during mechanical ventilation (maximal PIP) and duration of supplemental oxygen exposure did affect the relationships between $R_{\mathrm{rs}, \text { neoC }}$ and respiratory morbidity, and $R_{\mathrm{rs}, \text { neoC }}$ and $C_{\mathrm{rs}, 1 \text { year, }}$ respectively. Hence, correction for these factors seems important to reveal more pure aetiological relationships.

Since the compliance of the chest wall is very high in preterm infants, ${ }^{84} C_{\mathrm{rs}, \text { neo }}$ is predominantly affected by changing conditions of lung tissue. In severe RDS, surfactant deficiency together with alveolar deposition of hyaline membranes and interstitial oedema are known to decrease $C_{\mathrm{rs}}{ }^{43}$ As a consequence of decreased RDS severity, ${ }^{32}$ neonatal $C_{\mathrm{rs}}$ is less deteriorated and therefore not related to long-term respiratory outcome. In preterm infants developing CLD, Baraldi et al. ${ }^{15}$ showed that, instead of $R_{\mathrm{rs}}, C_{\mathrm{rs}}$ was strongly associated with forced expiratory flow ( $V_{\max , \mathrm{FRC})}$ at the age of 2 . However, $C_{\mathrm{rs}}$ was measured between 10 and 20 days of life. By that time, CLD usually shows serious progress, causing $C_{\mathrm{rs}}$ to decrease. Alternatively, the increased variability of early neonatal $C_{\mathrm{rs}}$ measurements make this a less sensitive tool in relation to respiratory outcome. The poor relationship between $C_{\mathrm{rs}, \text { neo }}$ and respiratory outcome in this study may well have been influenced by the use of surfactant. In 66 per cent of the infants in our study population, exogenous surfactant was instilled at a median age of 9 hours. Surfactant is known to immediately improve $C_{\text {rs }}$ values, ${ }^{45}$ and to decrease RDS severity. ${ }^{32}$ Postnatal corticosteroid administration did not show any confounding effect. Assessment of passive respiratory mechanics using the SBT according to Le Souef et al. ${ }^{150}$ is simple and can be easily performed in intubated preterm infants with minimal disturbance to the patient. We are well aware of the limitations of this technique, especially in intubated preterm infants. ${ }^{228}$ By using strict criteria recommended by the ERS, neonatal respiratory function results can be considered reliable. $\mathrm{CV}$ values of $\mathrm{R}_{\mathrm{rs}}$ and $C_{\mathrm{rs}}$ within subjects within measurements 
were acceptable, indicated by low median $C V$ values. $C V$ values of $R_{\mathrm{rs}}$ and $C_{\mathrm{rs}}$ within subjects between the daily measurements during the first 3 days of life were larger, especially for $C_{\mathrm{rs}}$ assessments. This indicates that $R_{\mathrm{rs}}$ and $C_{\mathrm{rs}}$ may change considerably during the first days of life.

More studies are needed to explore which factors contribute to intra-uterine lung development, postnatal lung function and respiratory outcome in the current surfactant era. We intend to follow our study population into childhood to explore future respiratory development in relation to early respiratory system mechanics.

To conclude, we found important causal relationships between early postnatal $R_{\mathrm{rs}}$ (but not $C_{\mathrm{rs}}$ and respiratory outcome during the first year of life in preterm ventilated infants. Relationships were not adversely affected by measures reflecting degree of prematurity, neonatal disease, and ventilator conditions. This indicates that inborn properties of the respiratory system have become increasingly important in relation to respiratory outcome instead of neonatal disease and treatment conditions. 



\title{
CHAPTER 7
}

\author{
Neonatal respiratory mechanics and \\ development of bronchial byperresponsiveness \\ in preterm infants
}

Yvonne Snepvangers

Peter de Winter

Huibert Burger

Hens Brouwers

Kors van der Ent 



\subsection{Abstract}

Background: In preterm ventilated infants, irreversible damage to the airway mucosa in the neonatal period might be related to the development of bronchial hyperresponsiveness (BHR) in subsequent years.

Aims: To evaluate whether neonatal indicators of long-term respiratory morbidity, respiratory system compliance $\left(C_{\mathrm{rs}}\right)$ and resistance $\left(R_{\mathrm{rs}}\right)$, were causally related to bronchial responsiveness at the age of two and whether these relationships were affected by other factors.

Study design: Mean neonatal $C_{\mathrm{rs}}$ and $R_{\mathrm{rs}}$ of the first 3 days of life were assessed using the single breath occlusion technique. Bronchial challenge tests were performed at 2 years of age. When wheezing occurred during chest auscultation or oxygen saturation decreased below 90 per cent, the provocative concentration of methacholine was recorded.

Subjects: Forty-five preterm infants of $<37$ weeks gestation, being mechanically ventilated within 24 hours after birth.

Results: Decreased neonatal $C_{\mathrm{rs}}$ was related to BHR ( $\beta$ per $\mathrm{ml} / \mathrm{kPa}, 0.061 ; 95 \%$ confidence interval, 0.019 to $0.103 ; p=0.006$ ). Correction was required for radiological gradation of respiratory distress syndrome, the maximal peak inspiratory pressure required during mechanical ventilation and postnatal corticosteroid therapy. Neonatal $R_{\mathrm{rs}}$, gestational age and birth weight were not related to subsequent BHR development.

Conclusion: In ventilated preterm infants, decreased neonatal $C_{\mathrm{rs}}$ was related to the development of BHR at the age of two.

\subsection{Introduction}

In preterm infants with chronic lung disease (CLD), bronchial hyperresponsiveness (BHR) in childhood is common. ${ }^{174,181,195,223}$ Also without development of CLD, bronchial responsiveness may be increased in preterm infants at school age. $78,94,195$ Irreversible damage to the airway mucosa due to inflammation and mechanical ventilation in the neonatal period is thought to cause BHR development in subsequent years. ${ }^{174}$ Data on BHR in infancy are scarce, as conventional methods required spirometry.

Alternatively, chest auscultation with pulse oximetry is easy to perform in young infants and seems to reveal valid results during bronchial challenge testing in older children. ${ }^{9,224}$ In preterm infants, neonatal respiratory system compliance $\left(C_{\mathrm{rs}}\right)$ and resistance $\left(R_{\mathrm{rs}}\right)$ are indicative of long-term respiratory morbidity, ${ }^{30,85,151,153,179,193}$ which may reflect BHR during childhood. ${ }^{174,181,195,223}$ More information about causal factors of BHR development is needed to elucidate the pathological process of BHR 
development in preterm infants. The purpose of this study was to investigate whether neonatal respiratory mechanics play a role in the aetiology of bronchial responsiveness in subsequent years in preterm ventilated infants and whether other relevant neonatal factors, like degree of prematurity, ventilator conditions, respiratory disease severity and therapeutic measures affected this relationship. To determine the clinical importance of BHR, we also investigated whether bronchial responsiveness was related to recent respiratory morbidity.

\subsection{Material and methods}

\subsubsection{Subjects}

Preterm infants of less than 37 weeks of gestation, who were admitted to our neonatal intensive care unit (NICU) between 28 July 1999 and 31 August 2000 and who were mechanically ventilated within 24 hours after birth, were studied prospectively. Infants with congenital anomalies were excluded from the study. The study was approved by the Medical Ethics Review Committee of our centre and parental informed consent was obtained.

Mechanical ventilation was started as a consequence of respiratory distress, when, despite supplemental oxygen and/or continuous positive airway pressure, the inspiratory oxygen fraction $\left(\mathrm{FiO}_{2}\right)$ remained above 0.7, the partial arterial carbon dioxide pressure exceeded $55 \mathrm{~mm} \mathrm{Hg}$, acidosis persisted with $\mathrm{pH}$ decreasing below 7.25 , or when serious apnoeas existed. Ventilator settings were adjusted to keep the arterial oxygen saturation of haemoglobin $\left(\mathrm{SaO}_{2}\right)$ between $90-95 \%$ and the partial arterial carbon dioxide pressure between $40-45 \mathrm{~mm} \mathrm{Hg}$. For mechanical ventilation, one of the following infant respirators was used: Stephanie (Stephan, Gackenbach, Germany), Bear BP2001 (Bear, Palm Springs, CA, USA), and Bourns BP200 (Bourns, Inc., Riverside, CA, USA). Respiratory function measurements were performed at neonatal age and bronchial challenge tests at the age of two. Additional information about respiratory morbidity within this period was collected from clinical charts during the neonatal period and questionnaires being completed by the parents at the end of the second year for the period after hospital discharge.

\subsubsection{Respiratory function tests}

We intended to perform measurements of passive respiratory system mechanics on each day during the first 3 days of life. Sometimes measurements could not be performed, because of clinical instability of the patient or unavailability of qualified personnel (i.e. two of the authors, Y.S. and J.P.d.W.). To optimize the total number of 
measurement results, mean $C_{\mathrm{rs}}$ and $R_{\mathrm{rs}}$ of the first 3 days of life were calculated. $C_{\mathrm{rs}}$ and $R_{\mathrm{rs}}$ were assessed according to the single breath occlusion technique (SBT) described by Le Souef et al. ${ }^{68,150} \mathrm{~A}$ heated Lilly-type pneumotachometer $(8410 \mathrm{~A}$, linear range $0-10 \mathrm{~L} / \mathrm{min}$, dead space $1.3 \mathrm{ml}$, resistance $0.94 \mathrm{kPa} / \mathrm{L} \cdot \mathrm{s}$ at $5 \mathrm{~L} / \mathrm{min}$; Hans Rudolph Inc., Kansas City, MO, USA) was placed between endotracheal tube and ventilator tubes. A pressure transducer (Honeywell, type 163PC01D75, Morristown, NJ, USA) and an IBM compatible personal computer with a Pentium processor running the software package Respiratory Analysis System Program (Version 007.17, Physio Logic, High Clere, Great Britain) were used to record signals at a sampling rate of $125 \mathrm{~Hz}$ using a 12 bit analogue-to-digital converter (LabMaster DMA, Scientific Solutions, Mentor, OH, USA). Mouth pressure and flow signals were obtained after calibration before each single measurement. For flow calibration, a calibrated rotameter (air 0-15 L/min, Medec, Aalst, Belgium) was used followed by a volume calibration using a precision syringe of $50 \mathrm{ml}$ containing the actual gas mixture. For pressure calibration, a pressure transducer tester (Veri-Cal 650-900, Utah Medical Products Inc., Midvale, Utah) was used. Leaks in the system or around the endotracheal tube were easily detected as mouth pressure decreased gradually during occlusion. With gentle manual compression of the trachea present leaks were minimized. Occluded breaths with tube leaks causing a decrease in occlusion pressure of $0.02 \mathrm{kPa}$ or more were excluded from further analysis.45,220 During occlusion, activity of inspiratory muscles is inhibited via stimulation of the airway receptors inducing the Hering-Breuer reflex. Breath analysis was considered unreliable, when no muscle relaxation was achieved. Muscle relaxation was assumed to be present when (1) during a short occlusion period of less than 0.5 second mouth pressure did reach a plateau for at least 0.1 seconds and pressure changes during this period showed a standard deviation (SD) of $0.01 \mathrm{kPa}$ or less, and (2) the expiration part of the flow volume curve was linear for at least 50 per cent of the volume to be expired. As a criterion for linearity, a correlation coefficient of 0.98 or more had to be achieved after exclusion of the final 5 per cent of the expired volume. We intended to use at least five occluded breaths meeting these criteria for calculation of a mean value for both respiratory system mechanics for each single measurement. These criteria complied with the European Respiratory Society recommendations. ${ }^{80}$ We are well aware of the limitations of these respiratory function measurements in intubated preterm infants. ${ }^{230}$ To evaluate internal validity of the tests, the intrasubject variabilities of $R_{\mathrm{rs}}$ and $C_{\mathrm{rs}}$ were studied by evaluating their coefficients of variance (CV) within each single measurement of all measurements meeting the European Respiratory Society recommendations. CV values within subjects between the measurements of the first 3 days were also assessed. 


\subsubsection{Bronchial challenge tests}

Bronchial challenge tests were carried out by one of the authors (Y.S.) at 2 years of age using a continuous inhalation tidal breathing method as described by Cockroft et al. ${ }^{34}$ Before the test was started, chest auscultation was performed. Isotonic saline was administered for 2 minutes from a nebulizer (Up-draft II nebulizer, Hudson Respiratory Care Inc., Temecula, CA), connected to a flow meter (Endomed Air $20^{\circ} \mathrm{C}$ 0-17 L/min, Endomed B.V., Didam, The Netherlands) delivering a flow rate of 6 $\mathrm{L} / \mathrm{min}$ out of the hospital circuit of compressed air into the nebulizer. Chest auscultation was repeated shortly after saline nebulization. If no wheeze was heard, a serial inhalation of doubling concentrations of methacholine was started from a concentration of $0.15 \mathrm{mg} / \mathrm{ml}$ every 5 minutes to a maximum concentration of 9.6 $\mathrm{mg} / \mathrm{ml}$ (i.e. $0.15,0.30,0.60,1.20,2.40,4.80$ and $9.60 \mathrm{mg} / \mathrm{ml}$ consecutively). Each methacholine concentration was nebulized for 2 minutes. Shortly after each inhalation, chest auscultation was performed by the same observer every time.

The response to the challenge was monitored by a pulse oximeter (Nellcor $\mathrm{N}$ 395, Mallinckrodt Europe B.V., 's-Hertogenbosch, The Netherlands) attached around a toe. Baseline values of $\mathrm{SpO}_{2}$ were attained after a short period of stabilization. The challenge was considered positive and terminated if an audible wheeze was present over the chest, if $\mathrm{SpO}_{2}$ fell below 90 per cent (for at least ten seconds) or if the final methacholine concentration of $9.6 \mathrm{mg} / \mathrm{ml}$ was reached, whatever occurred first. At that moment, the provocative concentration of methacholine (PC) was recorded, as was the $\mathrm{SpO}_{2}$. Children inhaled $800 \mu \mathrm{g}$ of Salbutamol via a Babyhaler to decrease bronchusconstriction. When children were wheezing before initiation of the test, their parents were kindly requested to come back for the test a few days or weeks later.

\subsubsection{Data analysis}

PCs were expressed as the number of doubling concentrations of methacholine and were transformed by taking the square root to accomplish a normal distribution. Relevant clinical neonatal factors were studied univariately in relation to PC, including gestational age, birth weight, maximal radiological gradation for respiratory distress syndrome (RDS) according to Giedion, ${ }^{86}$ duration of mechanical ventilation, number of days with a $\mathrm{FiO}_{2}$ of $>0.4$, maximal peak inspiratory pressure (PIP) required during ventilation, postnatal corticosteroid therapy, CLD development (oxygen dependency at 36 weeks postconceptional age), and $C_{\mathrm{rs}}$ and $R_{\mathrm{rs}}$.

Secondly, causal relationships based on a priori assumptions were investigated between neonatal mechanics and PC by linear regression analyses with PC as the dependent variable and $C_{\mathrm{rs}}$ and $R_{\mathrm{rs}}$ as alternate independent variables. Thirdly, we studied whether these relationships were affected by potentially confounding factors: 
gestational age, birth weight, maximal radiological RDS grade according to Giedion, ${ }^{86}$ duration of mechanical ventilation, maximal PIP required during ventilation, number of days requiring a $\mathrm{FiO}_{2}$ of $>0.4$, postnatal steroid therapy, CLD (oxygen dependency at 36 weeks post-conceptional age), familial history of asthma or atopy, and use of inhaled corticosteroids, number of colds, smoking by one or more household members, and the presence of pets within the household for at least 1 year. These factors were included separately into the initial regression models. Any factors affecting the regression coefficients of either $C_{\mathrm{rs}}$ or $R_{\mathrm{rs}}$ by more than $10 \%$ were considered important and were included in the models.

To study the clinical relevance of PC in our study population, we investigated whether PC was associated with occurrence of respiratory symptoms of coughing or wheezing of any duration and inhalation therapy, e.g. bronchodilators or corticosteroids, during the second year of life by logistic regression analyses. To compare PC values in our study with the values described in previous studies in which individual data were presented of children of similar ages, independent Students' $t$-tests were used. Statistical significance was defined as a $p$-value of less than 0.05 .

\subsection{Results}

Eighty-eight infants were recruited. Eleven of them died during the neonatal period at the NICU, leaving 77 for follow-up. Six infants died of respiratory insufficiency, four due to neurological problems and one due to circulatory problems. In seven of 77 infants, neonatal $C_{\mathrm{rs}}$ and $R_{\mathrm{rs}}$ assessments did not meet the criteria recommended by the European Respiratory Society. In $45(64 \%)$ of the 70 infants left, bronchial responsiveness was assessed at the age of two. Parents of 18 infants did not return for the bronchial challenge tests for various personal reasons or because they did not want to expose their children to the tests. In two additional cases, no reason was given. Four children did visit the hospital, but did not complete the challenge tests, and one child was tested 4 months late. The results of these children were excluded for further analysis. No clinically important differences existed between the original group of 70 patients and the group of 45 patients who were included for further analysis (Table 1). Information about occurrence of respiratory symptoms and use of inhalation medication during the second year of life was complete in 44 of 45 $(98 \%)$ infants.

In the majority of cases $(77 \%)$, bronchial challenge tests were terminated in the presence of at least an audible wheeze during chest auscultation. In $13 \%$ of cases this was accompanied by a decrease of $\mathrm{SpO}_{2}$ below 90 per cent. In $18 \%$ of cases, tests were terminated by a decrease of $\mathrm{SpO}_{2}$ below 90 per cent without an audible wheeze. In one child, the maximum concentration of $9.6 \mathrm{mg} / \mathrm{ml}$ was reached and in another case the test was terminated after reaching the fourth doubling concentration of 
Table 1. Patient characteristics in 70 infants who were initially available for follow-up compared to 45 infants with actual bronchial challenge test results.

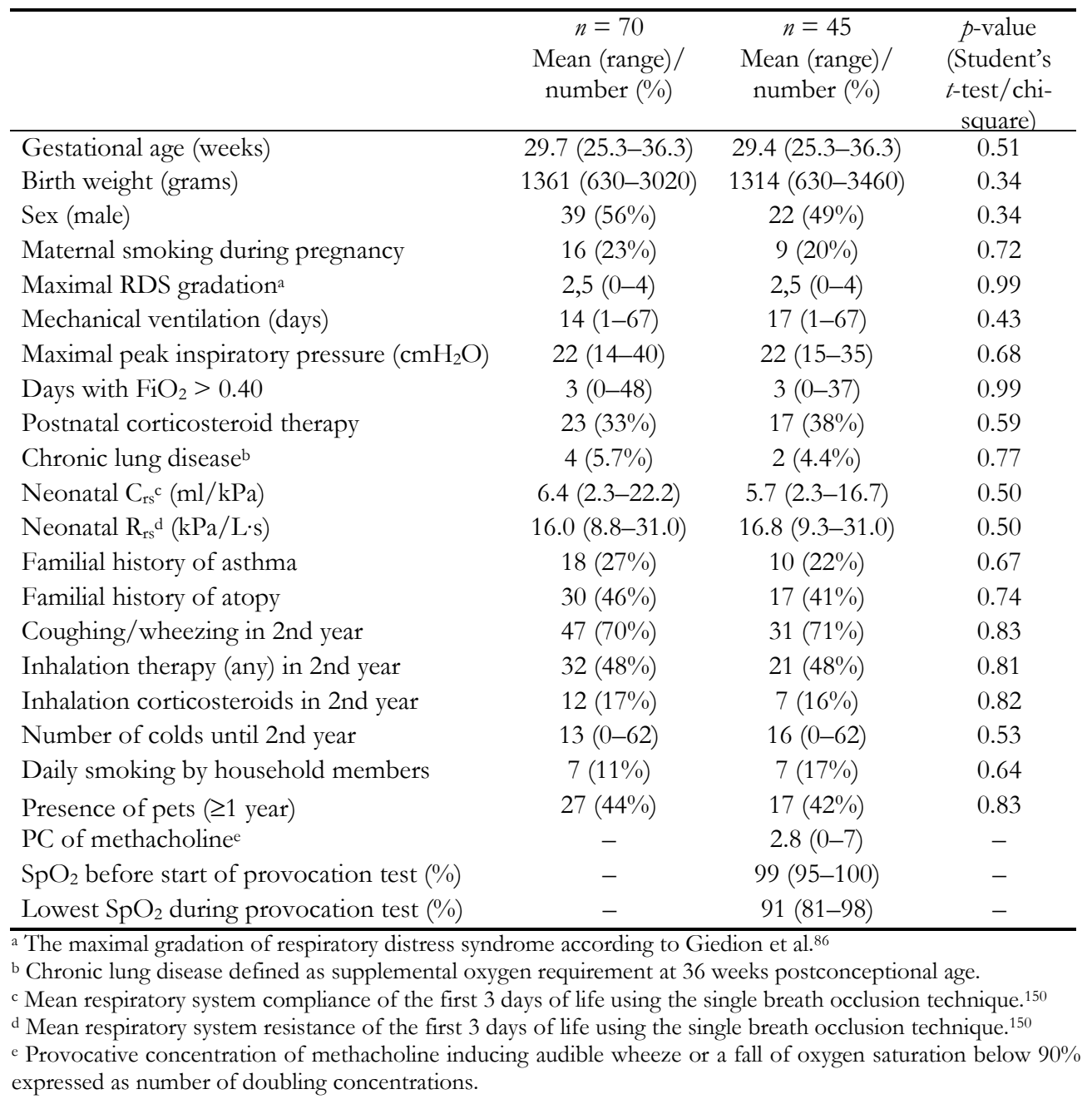

methacholine without any wheeze or $\mathrm{SpO}_{2}$ decrease, because the child did not longer accept the nebulizing mask near its face. $\mathrm{SpO}_{2}$ during the provocation tests did not descend below 80 per cent (Table 1).

According to univariate analyses, PC was significantly associated with maximal radiological RDS gradation according to Giedion ${ }^{86}$ and neonatal $C_{\mathrm{rs}}$ (Table 2). No associations were found between PC and gestational age, birth weight, maximal peak 
Table 2. Results of univariate regression analyses studying associations between neonatal factors and bronchial responsiveness at 2 years of age (dependent variable)

\begin{tabular}{|c|c|c|c|}
\hline Independent variable & $\beta$ per unit of independent & $95 \%$ confidence interval & $p$-value \\
\hline Gestational age (weeks) & 0.038 & $-0.020-0.096$ & 0.193 \\
\hline Birth weight (grams) & 0.0002 & $0.000-0.001$ & 0.147 \\
\hline Maximal RDS gradation ${ }^{a}$ & -0.141 & $-0.259--0.024$ & 0.020 \\
\hline Maximal PIPb $\left(\mathrm{cmH}_{2} \mathrm{O}\right)$ & 0.025 & $-0.009-0.059$ & 0.144 \\
\hline Mechanical ventilation (days) & -0.005 & $-0.013-0.003$ & 0.247 \\
\hline Days with $\mathrm{FiO}_{2}>0.40$ & 0.008 & $-0.015-0.032$ & 0.466 \\
\hline Postnatal corticosteroid therapy & -0.020 & $-0.327-0.287$ & 0.895 \\
\hline $\mathrm{CLD}^{\mathrm{c}}$ & -0.240 & $-0.958-0.479$ & 0.505 \\
\hline Neonatal Crs ${ }^{\mathrm{d}}(\mathrm{ml} / \mathrm{kPa})$ & 0.061 & $0.019-0.103$ & 0.006 \\
\hline Neonatal Rrse $(\mathrm{kPa} / \mathrm{L} \cdot \mathrm{s})$ & -0.021 & $-0.048-0.005$ & 0.111 \\
\hline
\end{tabular}

inspiratory pressure, duration of mechanical ventilation, number of days with $\mathrm{FiO}_{2}$ of $>0.4$, postnatal corticosteroid therapy, and CLD development. Neonatal $R_{\mathrm{rs}}$ was inversely related to PC, but not significantly. Correction for endotracheal tube size by adding this variable in the regression model did not change this $(\beta$ per $\mathrm{kPa} / \mathrm{L} \cdot \mathrm{s},-0.021$; $95 \%$ confidence interval (CI), -0.051 to $0.009 ; p=0.170)$.

Since decreased neonatal $C_{\mathrm{rs}}$ was significantly associated with decreased PC at the

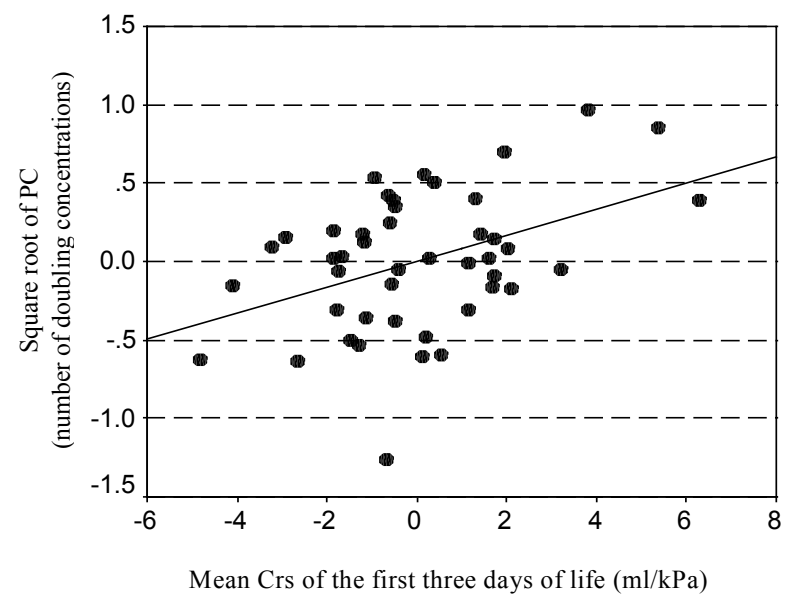

Figure 1. The association between neonatal $C_{\mathrm{rs}}$ and the provocative concentration (PC) of methacholine at the age of two after correction for RDS gradation, maximal PIP during mechanical ventilation and postnatal corticosteroid therapy $(\beta$ per $\mathrm{ml} / \mathrm{kPa}, 0.083$; $95 \%$ confidence interval, 0.026 to $0.140 ; p=0.006) . R^{2}=0.18$. 


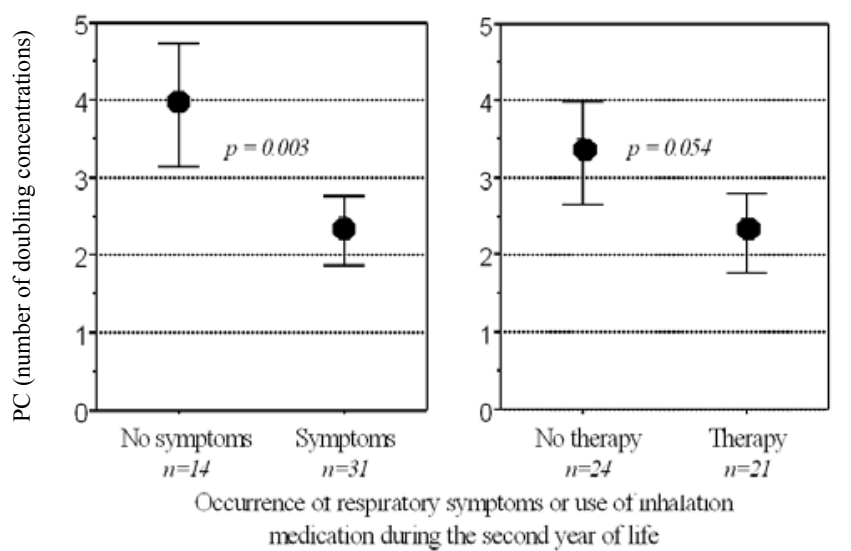

medication during the second year of life
Figure 2. Mean provoc-ative concentration (PC) of methacholine causing an audible wheeze or a fall of oxygen saturation below 90 per cent for infants with and without periods of respiratory symptoms and for infants who did and did not use inhalation medication during the second year of life. Error bars indicate the $95 \%$ confidence interval.

age of two, we further analyzed this association. RDS gradation substantially affected this association ( $\beta$ per $\mathrm{ml} / \mathrm{kPa}, 0.047 ; 95 \% \mathrm{CI},-0.009$ to $0.103 ; p=0.098$ ). After including maximal PIP into the model, neonatal $C_{\mathrm{rs}}$ became more strongly related to bronchial responsiveness $(\beta$ per $\mathrm{ml} / \mathrm{kPa}, 0.077 ; 95 \% \mathrm{CI}, 0.036$ to $0.118 ; p=0.000$ ). A similar effect was seen after including postnatal corticosteroid administration $(\beta$ per $\mathrm{ml} / \mathrm{kPa}, 0.076 ; 95 \% \mathrm{CI}, 0.030$ to $0.122 ; p=0.002)$. Figure 1 represents the most accurate form of the association between neonatal $C_{\mathrm{rs}}$ and PC, i.e. after correction for RDS gradation, maximal PIP and postnatal steroid therapy (partial plot). Due to this correction, $C_{\mathrm{rs}}$ values in Figure 1 do not represent the initial measured values and may even be negative.

Presence of respiratory symptoms of wheezing and coughing during the second year of life was significantly related to decreased PC at the age of 2 (odds ratio (OR), $0.05 ; 95 \% \mathrm{CI}, 0.01$ to $0.34 ; p=0.003$ ). The association between the use of inhaled bronchodilators and/or steroids during the second year of life and decreased PC was borderline significant (OR, 0.27; 95\% CI, 0.07 to 1.03; $p=0.054$ ) (Figure 2).

In the initial study population of 70 infants, 55 assessments of $C_{\mathrm{rs}}$ were obtained at day 1, 48 at day 2, and 45 at day 3 . Since in a few of these occasions non-linear expiratory flow patterns precluded reliable $R_{\mathrm{rs}}$ assessment, $53 R_{\mathrm{rs}}$ assessments were obtained at day 1, 45 at day 2 and 44 at day 3 . The median number of occluded breaths (range), on which results of each single measurement were based on, was 6 (312). In nine measurements the amount of five occluded breaths was not reached and results were based on four reliable occluded breaths in five measurements and three reliable occluded breaths in four measurements. Of all neonatal respiratory function measurements, the median $\mathrm{CV}$ (range) within subjects within measurements of $C_{\mathrm{rs}}$ was 7.7 (1.5-38.7) and of $R_{\mathrm{rs}}$ was 6.4 (1.8-51.3). CV values within subjects between 


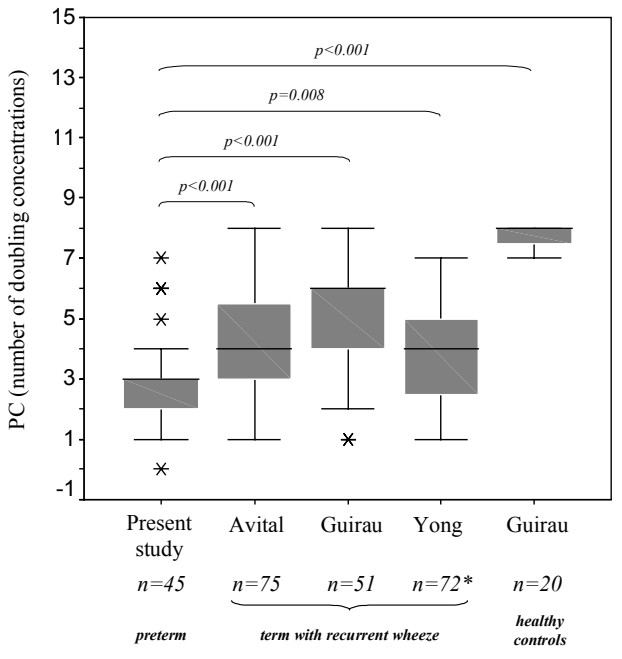

Figure 3. Provocative concen-trations of methacholine (PC) found in various studies using a similar protocol for bronchial challenge testing. Values are presented in a boxplot, in which each box represents 50 per cent of the cases (from 25th to 75th centile) and medians are marked by a horizontal line. The whiskers represent highest and lowest values within a range of 1.5 interquartile distance from the boxes. Values outside these ranges are marked as outliers.

measurements of the first 3 days of life were calculated in 68 per cent of the $C_{\mathrm{rs}}$ measurements and in 64 per cent of the $R_{\mathrm{rs}}$ measurements where respiratory function measures were based on more than one measurement. The median CV (range) between $C_{\mathrm{rs}}$ measurements was $24.9(0.8$ to 60.0$)$ and between $R_{\mathrm{rs}}$ measurements 15.6 (4.2 to 62.1).

\subsection{Discussion}

Decreased neonatal $C_{\mathrm{rs}}$ was significantly related to increased bronchial responsiveness at 2 years of age in preterm ventilated infants. Additional correction was required since RDS gradation, maximal PIP required during mechanical ventilation and postnatal corticosteroid therapy substantially affected this relationship. Bronchial challenge tests revealed clinically relevant results at the age of two, since bronchial responsiveness was increased in children with respiratory morbidity during the second year of life.

To our best knowledge, this is the first longitudinal study of preterm ventilated infants showing significant associations between neonatal respiratory function and bronchial responsiveness at the age of two. Without assessing neonatal respiratory function, other studies showed that childhood bronchial responsiveness was increased in preterm infants with and without CLD. $78,94,174,181,195,223$ In our study, only four per cent of the infants developed CLD. Hence, prematurity per se may be related to 
subsequent BHR development, which has been suggested before. ${ }^{195}$ Although impaired parenchymal development of the lung has been associated with CLD nowadays, ${ }^{57}$ it is predominantly caused by interruption of lung development at preterm birth and probably reflects mainly the degree of prematurity. ${ }^{115}$

Pulmonary immaturity after preterm birth is characterized by surfactant deficiency causing RDS with alveolar deposition of hyaline membranes and interstitial oedema. ${ }^{66}$ With increasing RDS severity $C_{\mathrm{rs}}$ decreases. ${ }^{53,112}$ Decreased neonatal $C_{\mathrm{rs}}$ might therefore point to a higher degree of pulmonary immaturity with an increased risk to develop BHR in subsequent years. During the infantile period, bronchial responsiveness decreases with age. ${ }^{178}$ Contractile forces of the airway smooth muscle, geometry of the airways, and impedance against which the airway smooth muscle must contract (cartilage and elastic recoil of the lung) change with age and affect airway reactivity. ${ }^{178}$ Additionally, forces of interdependence between pulmonary airways and parenchyma increase with age, limiting airway narrowing, ${ }^{239}$ and the number of cholinergic muscarinic receptors and their affinity for muscarinic agonists decrease with age while airway acetylcholinesterase activity increases. ${ }^{175}$ Hence, increased bronchial responsiveness after preterm birth may indicate that normal maturational changes in respiratory mechanics were, at least partly, absent. ${ }^{178}$ However, there is still some debate about whether bronchial responsiveness really decreases with age. ${ }^{280}$ Bronchial responsiveness may also be increased in infants with small airways, because narrowing of the airway lumen will be greater for the same degree of airway smooth muscle shortening. Although we found that increased neonatal $R_{\mathrm{rs}}$ was associated with BHR, this was not statistically significant. Neonatal $R_{\mathrm{rs}}$ was undoubtedly affected by endotracheal tube resistance. However, correction for tube size did not improve the association.

After correction for radiological RDS gradation, neonatal $C_{\mathrm{rs}}$ became less strongly associated with bronchial responsiveness. This suggests that radiological RDS gradation and $C_{\mathrm{rs}}$ contain independent, complementary information related to BHR development. Mechanical ventilation might cause irreversible damage to the airway mucosa in the neonatal period increasing the risk of BHR development. ${ }^{174}$ Independently of pulmonary disease, however, the level of end-inspiratory pressures within the pressure-volume curve during mechanical ventilation reflects the degree of lung recruitment and overdistention, and immediately affects $C_{\mathrm{rs}} .132$ This may explain why the association between $C_{\mathrm{rs}}$ and bronchial responsiveness was strengthened after correction for PIP. Infants with decreased neonatal $C_{\mathrm{rs}}$ are at increased risk of receiving postnatal corticosteroid therapy as a consequence of increased pulmonary disease severity. In our study, neonatal $C_{\mathrm{rs}}$ became more strongly associated with bronchial responsiveness after correction for postnatal corticosteroid therapy. Postnatal corticosteroid therapy may inhibit subsequent development of BHR by reducing initial lung damage. Dik et al. ${ }^{49}$ demonstrated that dexamethasone causes a downregulation of inflammation in preterm infants at risk of CLD development. 
However, they also found that fibroblast proliferation was not reduced after dexamethasone administration and probably will remain increased in most of these infants. By counteracting bronchusconstriction, fibrosis could be an additional explanation for reduced BHR development.

In international literature, there is a substantial deficit of studies investigating bronchial responsiveness in healthy children. According to the results in 20 healthy young children studied by Guirau et al.,98 bronchial responsiveness was increased (i.e. $\mathrm{PC}<5 \mathrm{mg} / \mathrm{ml}$ methacholine) in 44 of $45(98 \%)$ children in our study (Figure 3). Avital et al. ${ }^{9}$ studied 75 children at a mean age of 4.6 years ( 1 to 8 years) with clinical bronchial asthma, recurrent pneumonia, or chronic cough. Most of their children were older and $\mathrm{SpO}_{2}$ was not a criterion for challenge test termination. Both factors might explain their higher PC values. PC values in our study were also significantly lower compared to the results of Yong et al. ${ }^{274}$, who performed 72 bronchial provocation tests in 39 recurrently wheezing children at the same age using a similar protocol. Compared to the results of these studies bronchial responsiveness was substantially increased in our study population.

Assessment of passive respiratory mechanics according to Le Souef et al. ${ }^{150}$ is simple and can be easily performed in intubated preterm infants with minimal disturbance to the patient. We are well aware of the limitations of this technique, especially in intubated preterm infants. ${ }^{230}$ By using strict criteria recommended by the ERS, neonatal respiratory function results were reliable. CV values of $R_{\mathrm{rs}}$ and $C_{\mathrm{rs}}$ within subjects within measurements were acceptable, indicated by low median CV values. $\mathrm{CV}$ values of $C_{\mathrm{rs}}$ and $R_{\mathrm{rs}}$ within subjects between the daily measurements during the first 3 days of life were larger, especially for $C_{\mathrm{rs}}$ assessments, indicating that $C_{\mathrm{rs}}$ may change considerably during the first days of life in ventilated preterm infants.

Avital et al. ${ }^{9}$ showed that clinical grading of asthma was significantly related to degree of bronchial responsiveness. According to Guirau et al..$^{98}$ bronchial responsiveness was significantly increased in wheezing babies under 2 years of age compared to healthy controls. This was supported by our findings. There may be, however, some concern about the safety and applicability of the method we used to assess bronchial responsiveness in young children.282 Our results were similar to the results described by Springer et al., ${ }^{226}$ who found that termination of the bronchial provocation test was accompanied by at least the presence of audible wheeze in 81 per cent of young children with asthma (mean age 4.3 years), compared to 77 per cent in our study, and no severe desaturations were noted. In other studies was shown that occurrence of lung sounds agreed well with a 20 per cent fall in forced expiratory volume in one second during bronchial challenge tests in older children.9,224 Also, interobserver variability was highly acceptable with complete agreement between three observers in 80 per cent of cases. ${ }^{9}$ As was concluded by Springer et al., ${ }^{282}$ we think that the method that we used to assess bronchial responsiveness is safe, effective, easily applicable and clinically significant. However, further standardization and validation of bronchial 
challenge tests is required in both healthy and non-healthy children at different ages, but especially in young children.

One important limitation of this study is the low statistical power, because of the relatively small study population. However, infants studied during follow-up measurements at 2 years of age were representative of the initial group of recruited infants. Our results give, therefore, a first impression of determinants of bronchial responsiveness during lung maturation in preterm infants, but need further confirmation by larger studies.

In summary, in preterm ventilated infants, decreased neonatal $C_{\mathrm{rs}}$ was related to BHR at the age of two. BHR might reflect the absence of normal maturational changes during lung development as a consequence of early interruption of lung development at an immature stage at preterm birth. 


\section{CHAPTER 8}

Course of respiratory function in preterm ventilated infants during the first week and early years after birth

Yvonne Snepvangers

Peter de Winter

Kors van der Ent 



\subsection{Abstract}

Due to recent developments in perinatal care, the incidence of severe acute respiratory disease in preterm infants has decreased during the past 10 to 20 years. This may have important consequences for the respiratory course during the early years after birth. We explored tracking of respiratory function during the first week and first 2 years after birth in ventilated preterm infants.

Preterm infants of less than 37 weeks gestation with the requirement of mechanical ventilation within the first 24 hours after birth were recruited. Serial respiratory function tests were performed at days 1,2, 3 and 7, and at 1 year and 2 years of age. Respiratory system resistance and compliance were assessed during the neonatal period and at 1 year of age by applying the Single Breath Occlusion Technique. At 2 years of age, bronchial responsiveness was assessed by performing methacholine challenge tests.

In 80 infants, tests were performed during the neonatal period. Fifty-seven infants were tested at 1 year of age and 45 infants at 2 years of age. During the first week of life, a general deterioration of both $R_{\mathrm{rs}}$ and $C_{\mathrm{rs}}$ was noticed during the first 3 days of life followed by a gradual improvement towards the end of the first week. During the first year of life, deterioration of both measures improved and interindividual differences decreased considerably. Tracking of $R_{\mathrm{rs}}$ during this period was more obvious than tracking of $C_{\mathrm{rs}}$. Finally, bronchial responsiveness at 2 years of age was most consistently associated with $C_{\mathrm{rs}}$ course during the first year of life.

Courses of neonatal $R_{\mathrm{rs}}$ and $C_{\mathrm{rs}}$ during the first week of life reflected characteristic pulmonary pathology in ventilated preterm infants During the first year of life, $R_{\mathrm{rs}}$ showed better tracking than $C_{\mathrm{rs}}$, although it was only partially present. Interindividual differences tended to decline for both measures and an overall improvement was seen.

\subsection{Introduction}

In the past, neonatal and childhood respiratory function have been studied extensively in relation to preterm birth $^{78,94,179,193,243}$, CLD development ${ }^{15,19,70,85,89,91,137,}$ 160,193,219,256, and neonatal therapeutic interventions. ${ }^{94,151,196,275}$ Only one of these studies consequently performed serial respiratory function measurements in 24 infants, who developed CLD, during the first 2 years of life commencing in the neonatal period. ${ }^{15}$ It would therefore be interesting to summarize the results of chapters 6 and 7 of this thesis and show the complete course of respiratory function from the neonatal period until 2 years of age. Therefore, respiratory function results of ventilated preterm infants are presented that were performed at days 1,2, 3 and 7, and at 1 year and 2 years of age. $R_{\mathrm{rs}}$ and $C_{\mathrm{rs}}$ were assessed during the neonatal period and at 1 year of age 
by using the Single Breath Occlusion Technique described by Le Souef et al. ${ }^{150}$ At 2 years of age, bronchial responsiveness was assessed by applying methacholine provocation tests described by Cockroft et al. ${ }^{34}$ We investigated whether respiratory function course corresponded with expected pulmonary pathology during the first week of life in preterm ventilated infants. Additionally, tracking of respiratory function during the first 2 years of life was explored.

\subsection{Methods}

For the description of the subjects and respiratory function measurements, the reader is referred to Chapters 6 and 7 of this thesis.

\subsubsection{Data analysis}

Courses of $R_{\mathrm{rs}}$ and $C_{\mathrm{rs}}$ during the first week of life were presented for the whole study population as mean z-scores and their standard deviations (SD) of $R_{\mathrm{rs}}$ and $C_{\mathrm{rs}}$ at days $1,2,3$ and 7. $Z$-score calculations were based on reference values of healthy, non-intubated preterm infants described by Gappa et al. ${ }^{81}$ As a conse-quence of missing values due mainly to extubation before measurements were performed, the number of infants declined during this period. Results of $R_{\mathrm{rs}}$ were presented with and without correction for tube resistance, which was estimated in vitro at a flow rate of $50 \mathrm{ml} / \mathrm{s}$.

Secondly, tracking of $R_{\mathrm{rs}}$ and $C_{\mathrm{rs}}$ during the first year of life was presented by mean $z$-scores and their 95\% confidence intervals (CI) for each tertile of the study population. $Z$-score calculations were based on reference values of healthy term infants under 2 years of age described by Gutkowski et al. ${ }^{99}$ Tertiles were based on the median $R_{\mathrm{rs}}$ or $C_{\mathrm{rs}}$ value of the first 3 days of life, tertile 1 consisted of the lowest values and tertile 3 of the highest values measured. Finally, first-year tracking of $R_{\mathrm{rs}}$ and $C_{\mathrm{rs}}$ was studied in relation to bronchial responsiveness at 2 years of age. Means with $95 \%$ CIs were presented for the same tertiles.

In case results were normally distributed and variances within tertiles were equal for each tertile (according to Levene's test of homogeneity of variance), differences between tertiles were analysed by ANOVA analyses of variance, followed by a Bonferroni correction for multiple comparisons. If results were not normally distributed or Levene's test was significant, Kruskal-Wallis analyses were performed and, if significant, Mann-Whitney $U$-tests were executed subsequently to elucidate which tertiles significantly differed from each other. For comparison of patient characteristics also Chi-square tests were used when investigating differences of frequency of occurrence. 


\subsection{Results}

\subsubsection{Subjects}

Initially, 88 infants were recruited (Figure 1). Since during the first 3 days the results of $8(9 \%)$ infants were considered not reliable according to the recommendations of the European Respiratory Society (ERS), 80 infants were included for further analyses. 10/80 (13\%) infants died during the neonatal period, six infants died of respiratory insufficiency, three due to neurological problems and one due to circulatory problems. Parents of 11 infants decided not to return for follow-up measurements for various personal reasons, in one additional case no reason was given, and one infant was tested 3 months late and therefore excluded for further analysis. Parents of 19 infants did not return for the bronchial challenge tests for various personal reasons or because they did not want to expose their children to this kind of tests. In two additional cases, no reason was given. Three children did not complete the challenge tests, and one child was tested 4 months late and therefore excluded for further analysis. Patient characteristics for all groups of children are presented in Table 1.

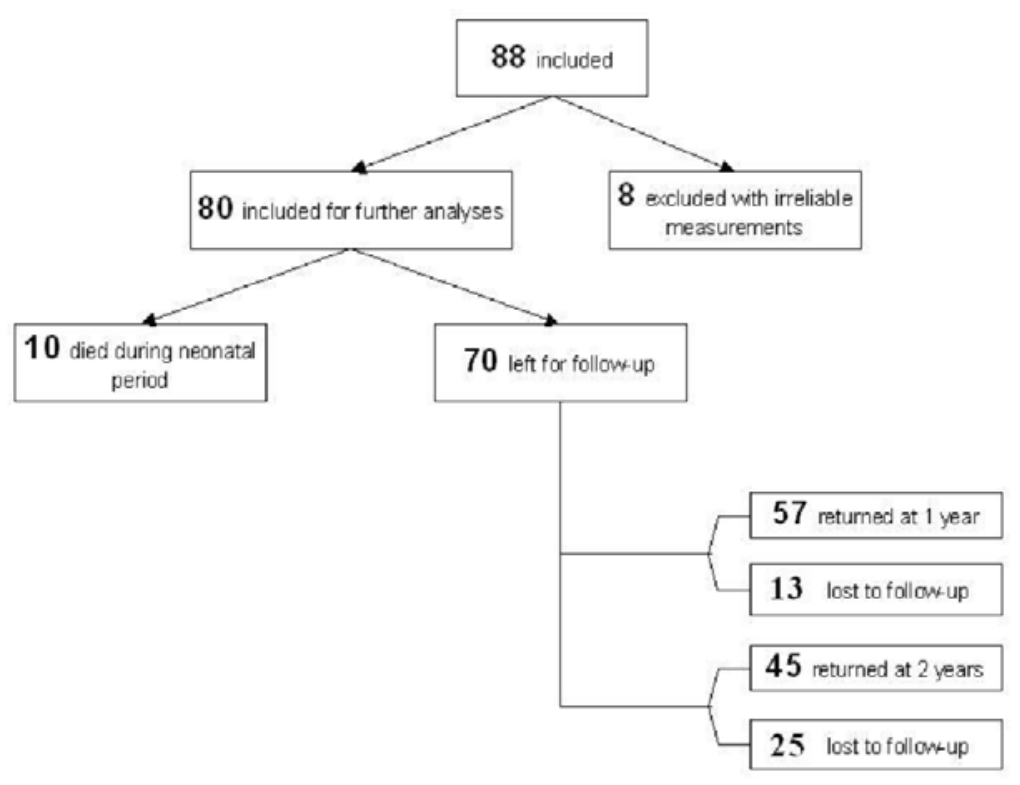

Figure 1. Patient selection and dropouts. 


\section{Course of respiratory function in early life}

Table 1. Patient characteristics of different selections of infants based on availability of respiratory function measurements during the neonatal period $(n=80)$, at 1 year of age $(n=57)$, and at 2 years of age $(n=45)$. Data are presented as medians (range) or as numbers (percentage).

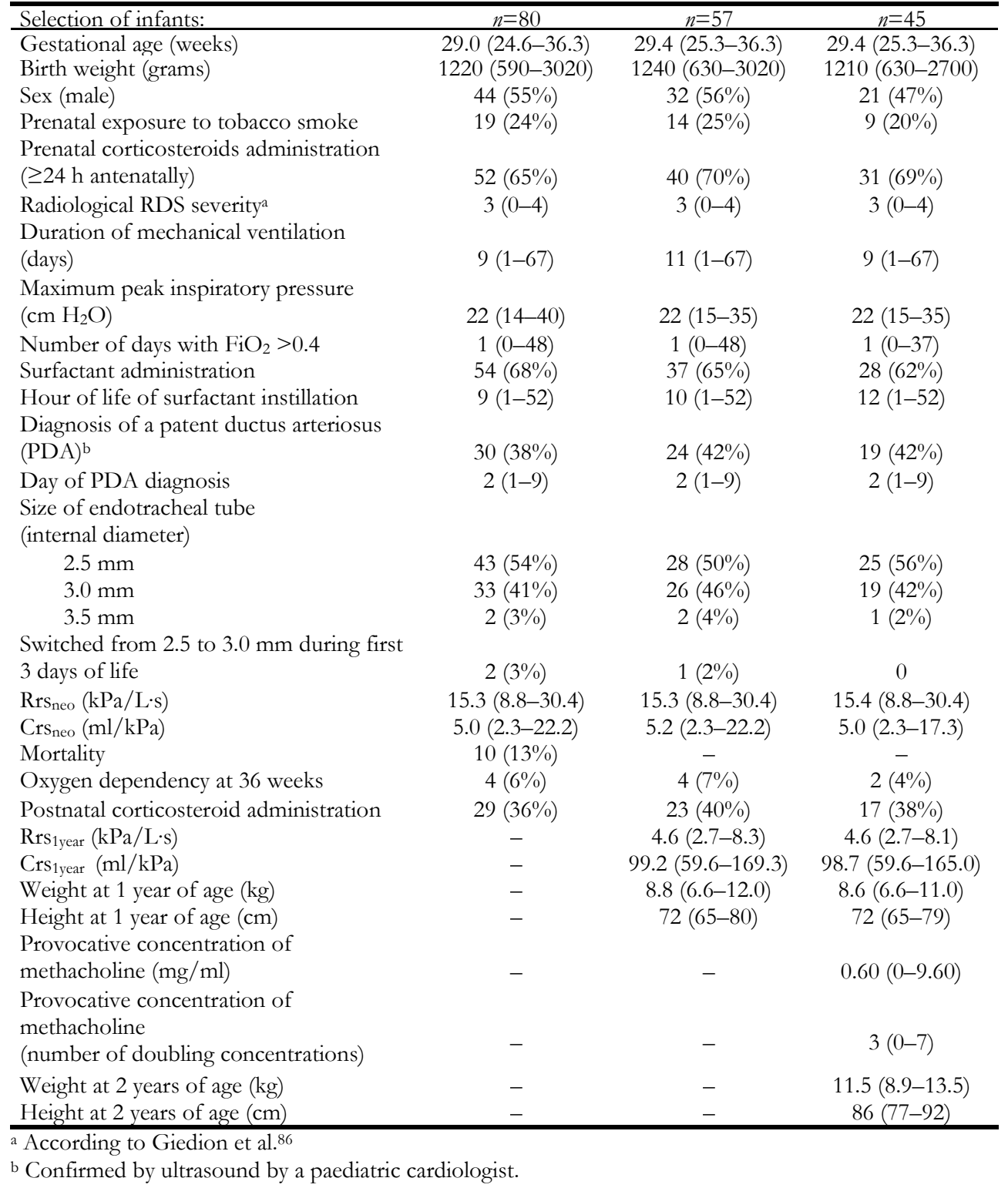



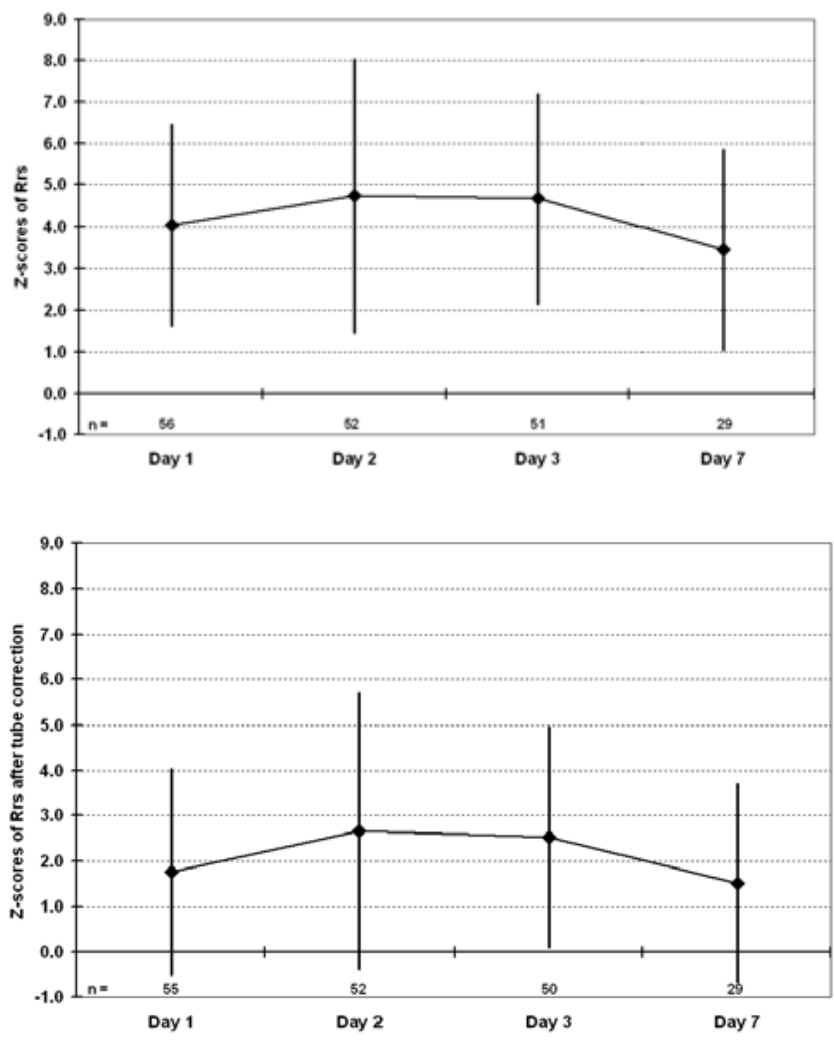

A.

Without correction for tube size.
B.

With correction for tube size.

Figure 2. $R_{\mathrm{rs}}$ course during the first week of life. Results are presented as means of the $z$-scores according to the results of Gappa et al. in healthy preterm infants. ${ }^{81}$ Error bars indicate $95 \%$ confidence intervals of the means.

\subsubsection{Neonatal respiratory function}

Results regarding the first week of life are presented as z-scores in Figures 2 and 3 for $R_{\mathrm{rs}}$ and $C_{\mathrm{rs}}$, respectively. $R_{\mathrm{rs}}$ results were presented both with and without correction for tube size. Of the results with tube correction, a few results were missing, because of wrong assessment of the length of the endotracheal tube. The results for the whole group of infants were substantially less deteriorated after tube correction compared to the results without tube correction. However, interindividual 


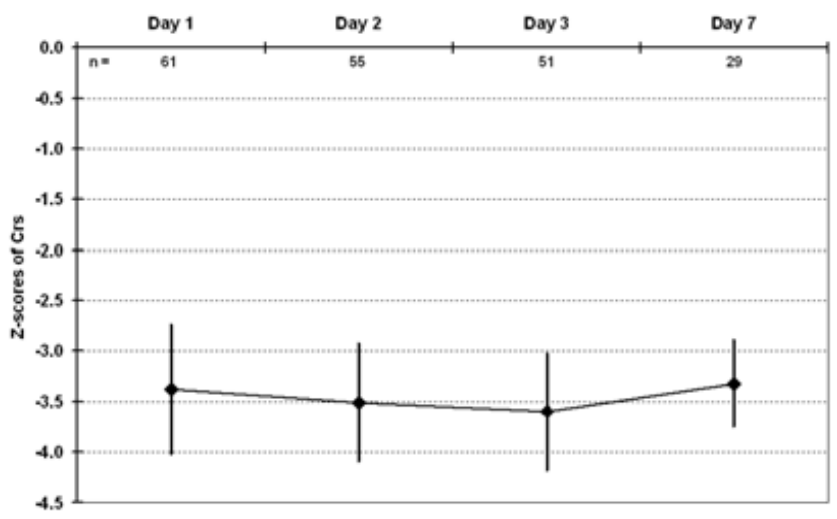

Figure 3. $C_{\mathrm{rs}}$ course during the first week of life. Results are presented as means of the $z$-scores according to the results of Gappa et al. in healthy preterm infants. ${ }^{81}$ Error bars indicate $95 \%$ confidence intervals of the means.

differences were relatively similar. During the first week of life, a deterioration of both $R_{\mathrm{rs}}$ and $C_{\mathrm{rs}}$ was seen within the first 3 days of life followed by an improvement towards day 7.

Table 2. Patient characteristics of each $R_{\mathrm{rs}}$ tertile without tube correction (mean $(95 \%$ confidence interval of mean)/number (percentage))

\begin{tabular}{lccc}
\hline & Tertile 1 & Tertile 2 & Tertile 3 \\
\hline Gestational age (weeks) & $31.0(29.9-32.2)^{* * *}$ & $29.5(28.5-30.6)$ & $28.5(27.6-29.4)^{*}$ \\
Birth weight (g) & $1615(1390-1840)^{* * *}$ & $1340(1112-1568)$ & $1131(985-1277)^{*}$ \\
Body weight at 1 yr (kg) & $9.6(9.1-10.0)^{* * *}$ & $8.7(8.0-9.4)$ & $8.4(7.9-8.9)^{*}$ \\
Body weight at 2 yrs (kg) & $11.8(11.2-12.3)$ & $11.0(10.4-11.6)$ & $11.2(10.6-11.8)$ \\
Antenatal corticosteroid & & & \\
therapy & $14(61 \%)$ & $16(67 \%)$ & $15(65 \%)$ \\
Maternal smoking & $6(26 \%)$ & $6(25 \%)$ & $4(17 \%)$ \\
RDS gradation & $2.1(1.5-2.7)$ & $2.7(2.2-3.2)$ & $2.7(2.1-3.2)$ \\
CLD & $3(13 \%)$ & $1(4 \%)$ & - \\
PDA & $10(44 \%)$ & $5(21 \%)$ & $12(52 \%)$ \\
Mechanical ventilation (days) & $11.8(4.2-19.4)^{* * *}$ & $12.3(7.4-17.2)$ & $19.1(12.3-25.9)^{*}$ \\
Supplemental oxygen (days) & $15.3(5.1-25.4)$ & $17.8(8.9-26.8)$ & $20.5(14.2-26.8)$ \\
Maximal peak inspiratory & $21.4(19.9-22.9)$ & $22.7(20.5-24.8)$ & $23.3(21.0-25.5)$ \\
pressure (cmH $\left.{ }_{2} \mathrm{O}\right)$ & & & \\
Surfactant therapy & $13(57 \%)$ & $14(58 \%)$ & $19(83 \%)$ \\
Postnatal corticosteroid & $5(22 \%)$ & $9(38 \%)$ & $9(39 \%)$ \\
\hline
\end{tabular}

${ }^{*} p<0.05$ compared to tertile 1 .

$* * * p<0.05$ compared to tertile 3 . 
Table 3. Patient characteristics of each $C_{\mathrm{rs}}$ tertile (mean ( $95 \%$ confidence interval of mean)/ number (percentage))

\begin{tabular}{lccc}
\hline & Tertile 1 & Tertile 2 & Tertile 3 \\
\hline Gestational age (wk) & $30.3(29.0-31.6)^{* * *}$ & $29.1(28.1-30.1)^{* * *}$ & $29.7(28.7-30.7)^{\#}$ \\
Birth weight (g) & $1587(1351-1822)^{* * *}$ & $1192(1032-1351)^{* * *}$ & $1314(1085-1543)$ \\
Body weight at 1 yr (kg) & $9.2(8.6-9.8)$ & $8.6(8.1-9.1)$ & $8.8(8.1-9.5)$ \\
Body weight at 2 yrs (kg) & $11.6(11.0-12.1)$ & $11.1(10.4-11.7)^{* * *}$ & $11.4(10.7-12.0)^{* *}$ \\
Antenatal corticosteroid & & & \\
therapy & $13(57 \%)^{\#}$ & $15(63 \%)^{*}$ & $17(74 \%)^{*}$ \\
Maternal smoking & $6(26 \%)$ & $4(17 \%)$ & $6(26 \%)$ \\
RDS & $3.3(3.0-3.6)^{* * *}$ & $2.5(2.1-3.0)^{* * *}$ & $1.6(1.0-2.2)^{\#}$ \\
CLD & $3(13 \%)$ & - & $1(4 \%)$ \\
PDA & $13(57 \%)^{\#}$ & $12(50 \%)^{*}$ & $2(9 \%)^{*}$ \\
Mechanical ventilation & & & \\
(days) & $13.2(8.3-18.2)^{* * *}$ & $20.2(12.2-28.3)$ & $9.4(4.0-14.9)^{*}$ \\
Supplemental oxygen & & & \\
(days) & $21.8(10.9-32.6)^{\#}$ & $20.3(12.9-27.7)^{*}$ & $11.3(4.8-17.9)^{*}$ \\
Maximal peak inspiratory & & & \\
pressure $\left(\mathrm{cm} \mathrm{H}_{2} \mathrm{O}\right)$ & $24.3(22.1-26.4)$ & $22.8(21.7-23.8)^{* * *}$ & $20.3(18.0-22.6)^{* *}$ \\
Surfactant therapy & $22(96 \%)^{* * *}$ & $18(75 \%)^{* * *}$ & $6(26 \%)^{\#}$ \\
Postnatal corticosteroid & $10(44 \%)^{* * *}$ & $8(33 \%)^{* * *}$ & $5(2)^{\# *}$ \\
\hline
\end{tabular}

${ }^{*} p<0.05$ compared to tertile $1 ;{ }^{* *} p<0.05$ compared to tertile 2 .

$* * * p<0.05$ compared to tertile 3; \#p<0.05 compared to all other tertiles.

\subsubsection{Respiratory function during infancy}

The study population was divided in three tertiles according to the median $R_{\mathrm{rs}}$ or $C_{\text {rs }}$ of the first 3 days of life. Patient characteristics of each tertile are presented in Tables 2 and 3. Main differences between $R_{\mathrm{rs}}$ tertiles concerned factors indicating degree of prematurity, like gestational age and birth weight. Although these factors were also different between $C_{\mathrm{rs}}$ tertiles, factors indicating respiratory disease severity, therapeutic interventions and complications showed also significant differences regarding $C_{\text {rs }}$ tertiles.

In Figure 4 is shown that tracking of $R_{\mathrm{rs}}$ was present during the first year of life, although two of the three tertiles were not distinguishable. Tracking of $C_{\mathrm{rs}}$ was not obvious during this period (Figure 5). The present neonatal deterioration of both $R_{\mathrm{rs}}$ and $C_{\mathrm{rs}}$ decreased during the first year of life, although this was not so obvious for the first $R_{\mathrm{rs}}$ tertile. Neonatal tertiles were significantly different, but these intertertile differences declined considerably during the first year of life. The rank order of the tertiles concerning bronchial responsiveness at 2 years of age, however, was inconsistent with respect to $R_{\mathrm{rs}}$ tertiles. In relation to $C_{\mathrm{rs}}$, bronchial responsiveness increased more consequently with decreasing $C_{\mathrm{rs}}$ values. 


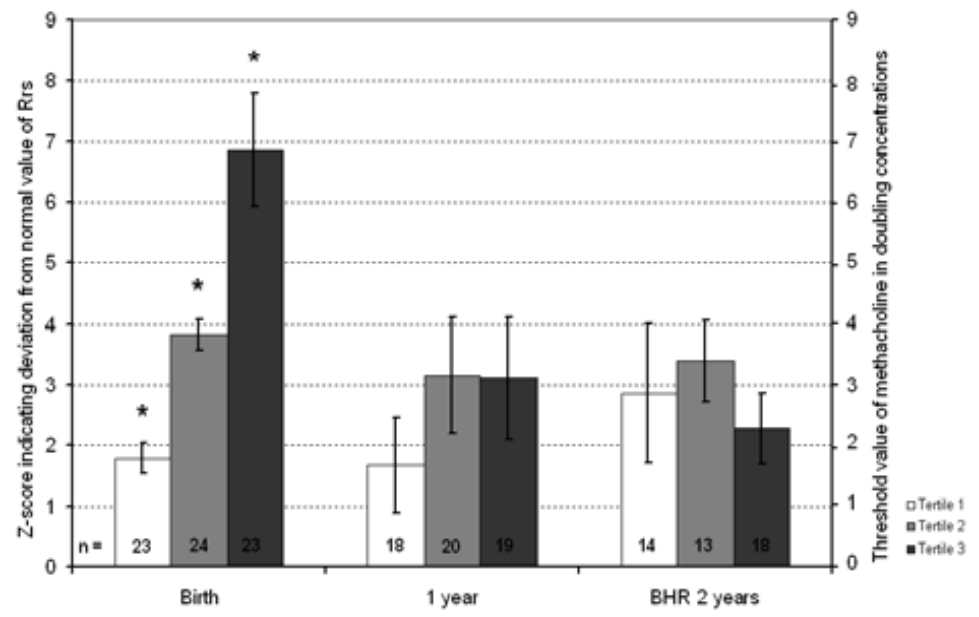

Figure 4. $R_{\mathrm{rs}}$ course during the first year of life combined with bronchial responsiveness at 2 years of age. Results are presented as means of tertiles of the study population based on median $R_{\mathrm{rs}}$ values during the first 3 days of life (bars in different gray scales). Error bars indicate 95\% confidence intervals of the means. *Significantly different from other tertiles at the same day according to the Mann-Whitney $U$ - test.

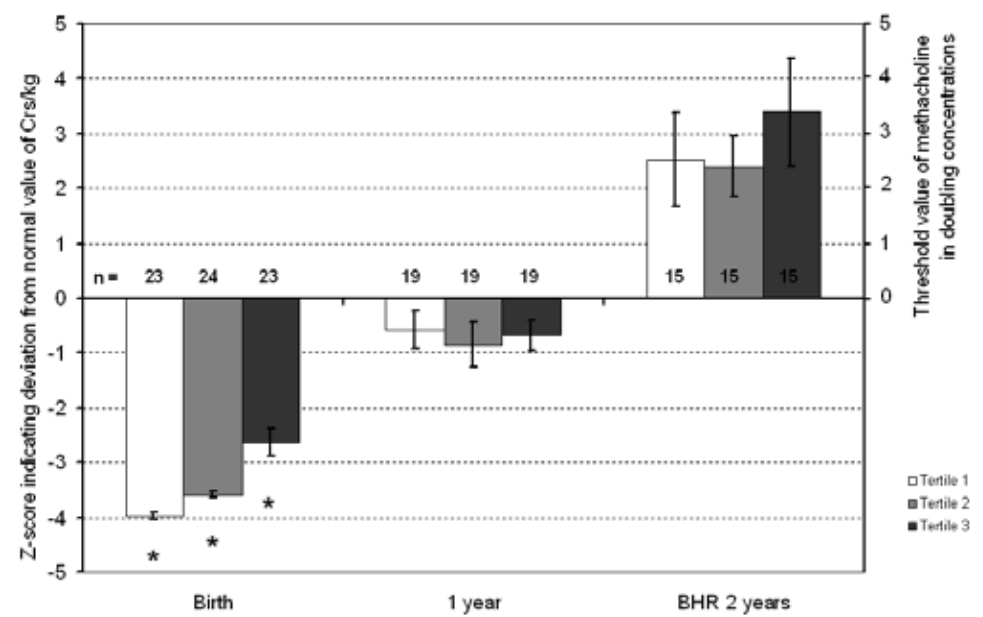

Figure 5. $C_{\mathrm{rs}}$ course during the first year of life combined with bronchial responsiveness at 2 years of age. Results are presented as means of tertiles of the study population based on median $C_{\mathrm{rs}}$ values during the first 3 days of life (bars in different gray scales). Error bars indicate $95 \%$ confidence intervals of the means. $*$ Significantly different from other tertiles at the same day according to the Mann-Whitney $U$-test. 


\subsection{Discussion}

In this study of ventilated preterm infants, course of respiratory function was studied during the first 2 years of life. During the first week of life, a general deterioration of both $R_{\mathrm{rs}}$ and $C_{\mathrm{rs}}$ was noticed during the first 3 days of life followed by a gradual improvement towards the end of the first week. During the first year of life, deterioration of both measures improved and intertertile differences decreased considerably. Tracking of $R_{\mathrm{rs}}$ during this period was more obvious than tracking of $C_{\mathrm{rs}}$. Finally, bronchial responsiveness at 2 years of age was most consistently associated with $C_{\mathrm{rs}}$ course during the first year of life.

To our best knowledge, this study is unique with respect to its concept of serial respiratory function assessments during the first 2 years of life in a group of ventilated preterm infants in the current surfactant era. Not only preterm infants developing CLD, but all preterm infants, in whom mechanical ventilation was initiated, were considered at risk of long-term respiratory morbidity. During the first week of life, respiratory function course showed a recognizable pattern and, during the early years after birth, a certain degree of tracking of respiratory function was present.

Courses of both $R_{\mathrm{rs}}$ and $C_{\mathrm{rs}}$ during the first week of life can be attributed to the course of pulmonary disease that is commonly seen in ventilated preterm infants. Respiratory condition often deteriorates during the first few days of life as a consequence of alveolar surfactant depletion and development of RDS. After symptoms and signs of RDS have reached a peak within 3 days, ${ }^{138}$ the respiratory condition normally shows a gradual improvement. When complications do not occur, infants are often extubated within the first week of life. Since, in this study, respiratory function measurements were only performed in intubated infants, a selection bias is likely, leading to a potential underestimation of respiratory function at mainly day 7 . Nevertheless, respiratory function showed a general improvement at day 7 compared to the first 3 days of life. Among those still intubated at day 7 are infants recovering from RDS, who will be extubated within the next few days, as well as infants developing CLD, who will remain intubated for a prolonged period. Irrespective of pulmonary pathology, preterm infants may also remain intubated as a consequence of immature breathing control mechanisms resulting in severe apnoeas.

$C_{\mathrm{rs}}$ results during the first week of life were decreased compared to previous findings in extremely preterm infants. ${ }^{136}$ In extremely preterm infants, RDS is often less severe ${ }^{58,129}$ and the chest wall more compliant. ${ }^{84}$ Interestingly, compared to findings in infants of 10 and 20 days old with established CLD, ${ }^{15}$ neonatal $C_{\text {rs }}$ results in our study were similar, but $R_{\mathrm{rs}}$ results were decreased. Hence, progression to chronic lung disease probably mainly affects $R_{\mathrm{rs}}$ by increased pulmonary oedema, increased smooth muscle mass, airway plugging with cellular debris, and increased mucus production. 85,188 


\section{Course of respiratory function in early life}

The improvement of both $R_{\mathrm{rs}}$ and $C_{\mathrm{rs}}$ in these infants during the first year of life can be attributed to excessive lung growth and to the continuous process of repair and remodelling, ${ }^{192}$ especially in infants with CLD. Extensive alveolarization and thinning of the alveolo-capillary membrane are the main factors increasing $C_{\mathrm{rs}}$, despite a considerable decrease of thoracic wall compliance. Since airway branching is normally complete at approximately the 16th week of gestation, ${ }^{202} R_{\mathrm{rs}}$ decreases by enlargement of the existing airways, changes of airway wall characteristics ${ }^{72}$ and structural changes in lung parenchyma. ${ }^{199}$

Although previous findings showed increased $R_{\mathrm{rs}}$ values between 10 to 20 days of life compared to neonatal values in our study, results at 2 years of age were similar, ${ }^{15}$ suggesting that long-term consequences of CLD may resemble long-term consequences in preterm infants without CLD. A potential confounding factor is postnatal administration of corticosteroids. Besides the effects of lung growth, intertertile $C_{\mathrm{rs}}$ differences at 2 year of age may be decreased by limited development of lung damage in the lowest $C_{\mathrm{rs}}$ tertile due to administration of postnatal corticosteroids. Several recent studies described a deterioration of airway function during the first year of life in preterm infants developing $\mathrm{CLD}^{117}$ and those without neonatal respiratory disease. ${ }^{119}$ Since in these studies maximal expiratory flow at functional residual capacity ( $V_{\text {maxFRC }}^{\prime}$ was studied, mainly representing characteristics of small intrathoracic airways, results were not comparable to the $R_{\mathrm{rs}}$ data of our study, which mainly represented characteristics of the large central airways ${ }^{72}$ and lung parenchyma. ${ }^{199}$ In our study, all $R_{\mathrm{rs}}$ tertiles showed improvement of the $z_{-}$-scores during the first year of life, probably mainly because of structural changes of lung parenchyma. ${ }^{199}$

Bronchial responsiveness increased most consistently with increasing $C_{\mathrm{rs}}$ values. Neonatal $C_{\mathrm{rs}}$ predominantly depends on parenchymal lung characteristics. The most important factors affecting lung parenchyma early after preterm birth are the development of RDS and degree of surfactant deficiency. The association between neonatal $C_{\mathrm{rs}}$ and RDS severity was confirmed by our finding that several factors related to RDS severity, like radiological RDS gradation, exogenous surfactant therapy, and maximal PIP were significantly different between neonatal $C_{\mathrm{rs}}$ tertiles. Hence, BHR development is probably associated with RDS severity and subsequent therapeutic measures.

Despite an initial recruitment of 88 ventilated preterm infants, only 57 and 45 infants were re-evaluated at 1 year and 2 years of age, respectively, because of neonatal mortality, unreliability of neonatal respiratory function assessments and refusal of parents to participate in follow-up measurements. The power of the study was therefore limited. However, infants studied during follow-up measurements at 1 year and 2 years of age were representative of the initial group of recruited infants. Unfortunately, $R_{\mathrm{rs}}$ and $C_{\mathrm{rs}}$ measurements were not repeated at 2 years of age, because sedation with chloral hydrate is increasingly unsuccessful at this age. Nevertheless, 
performing methacholine provocation test while awake added relevant information regarding the respiratory course in these children.

In conclusion, courses of neonatal $R_{\mathrm{rs}}$ and $C_{\mathrm{rs}}$ reflected characteristic pulmonary pathology in ventilated preterm infants during the first week of life. An initial deterioration, caused by surfactant depletion and RDS development, is followed by a gradual improvement as infants recover from RDS. During the first year of life $R_{\mathrm{rs}}$ showed better tracking than $C_{\mathrm{rs}}$, although it was only partially present. Interindividual differences tended to decline for both measures and an overall improvement was seen. 



\section{CHAPTER 9}

General discussion and future perspectives $\infty$

Algemene beschouwing en toekomstperspectief 



\subsection{Introduction}

The principle scope of the prospective cohort study described in this thesis was to investigate respiratory function during the neonatal period and early years after birth in ventilated preterm infants. Since the implementation of mechanical ventilation in the 1950s, survival of preterm infants has increased substantially and, as a consequence, physicians were confronted with unknown morbidity related to organ immaturity in a new patient population. Due to subsequent improvements in neonatal care, respiratory morbidity has changed considerably since then. The results of the studies described in this thesis will expand our knowledge on currently important clinical and mechanical determinants of early respiratory course in ventilated preterm infants.

\subsection{Limitations of respiratory function in mechanically ventilated preterm infants}

Possibilities for respiratory function assessments are limited in mechanically ventilated preterm infants. (Chapter 2) This is due mainly to the impaired physical condition of ventilated preterm infants and the specific environment at the neonatal intensive care unit. Because of clinical instability due to immaturity, small events may cause serious deterioration of the physical condition in these infants and measures to limit the number of moments of patient disturbance are thus of extreme importance. Therefore, respiratory function testing for research purposes should preferably be scheduled immediately after routine nursery care. Furthermore, equipment for respiratory function tests have to meet strict criteria with respect to dead space and resistance in order to minimise extra effort in breathing.

Besides limitations proceeding from the impaired physical condition of ventilated preterm infants, respiratory functions tests are limited by the specific environmental conditions. In intubated infants, the endotracheal tube is known to considerably increase the total resistance measured. ${ }^{152}$ Since in vitro assessments of endotracheal tube resistance do not correspond well with in vivo situations, ${ }^{152,164}$ correction of respiratory system resistance was not standard practice in the studies of this thesis. During mechanical ventilation, non-linearity of the pressure-volume relationships due to alveolar collapse toward end-expiration or overdistention of some lung units toward end-inspiration may affect actual respiratory function. Expiration into a ventilator system with maintained positive end-expiratory pressure (PEEP) will also affect respiratory function results, but loss of PEEP will often have unwanted adverse clinical implications in these vulnerable infants. Another important issue is the effect of the physical properties of ventilator gases on flow measurements by pneumotachometers, as was shown with respect to oxygen concentration in Chapter 3. 
Previous studies have demonstrated the effect of relative humidity, temperature, and pressure of gases on flow measurements $21,47,69,74,92,96,97,110,212,248,250,263,269$, but also of ventilator tubing characteristics and geometry. ${ }^{142}$ Effects are minimized when during calibration procedures environmental conditions correspond with measurement conditions or adequate correction is performed afterwards. Mechanical ventilators also depend on flow measurements by a pneumotachometer. Although in recently developed neonatal ventilators, algorithms are incorporated that correct for body temperature and pressure saturated with water vapour (BTPS), no correction is included for actual oxygen concentration and flow rate. In this thesis, correction factors for these effects were provided under specified calibration conditions. Other limitations for respiratory function testing in preterm ventilated infants include the inability to co-operate, dependence on incubator and ventilator, and the inability of transporting the patient from the neonatal intensive care unit to the department of paediatric pulmonology, where specialised equipment is available.

\subsection{Importance of respiratory function in neonatal respiratory course in ventilated preterm infants}

In clinical practice, several measures are used to indicate the actual respiratory condition in ventilated preterm infants. Chest radiographs are among others, like bloodgas analysis, pulse oximetry, ventilator conditions, and continuous monitoring of breathing movements, commonly used for this purpose. Although they can be easily applied without disturbance of the patient, interobserver agreement is known to be poor. ${ }^{213}$ In order to improve this, several scoring systems have been developed, in which specifically described radiographic aspects have to be scored according to a rank order of severity. Scores of different aspects can be summarized to calculate total scores, which have been found to associate with severity of respiratory distress syndrome (RDS) ${ }^{86,154}$ and clinical respiratory outcome. ${ }^{95,242,277}$ Despite these attempts to improve interobserver agreement, results in Chapter 4 indicate a poor interobserver agreement of the radiological scoring systems when applied during the first week of life in ventilated preterm infants. Although some of the scoring systems were not meant to be applied this early, this did not seem to be the most important cause, since other scoring systems, which were actually meant to be used this early, also showed poor agreement between observers. Furthermore, no associations were found between radiological scores and actual respiratory function. Poor interobserver agreement is a plausible cause, but the difference of pulmonary pathological aspects being accentuated by respiratory function and chest radiography may also play an important role.

Besides indicating the actual respiratory condition, early prediction of individual neonatal respiratory course is important for adequate neonatal care. In infants at 
increased risk of respiratory problems during the admission period at the NICU, increasing the priority share of measures to prevent development of pulmonary damage early after birth may improve respiratory outcome. In Chapter 5, reliable prediction models were presented for postnatal corticosteroid administration and prolonged mechanical ventilation, two important aspects of neonatal respiratory outcome in ventilated preterm infants. Previous studies mainly described prediction models for CLD development ${ }^{91}$ at 28 days ${ }^{19,62,89,160,210,215,256,270}$ or 36 weeks $^{87,95,160,214}$ and only a few were able to be applied within 2 days after birth. ${ }^{70,137,154}$ However, since the latter studies were performed before the surfactant era, results were not attributable to current practice. Furthermore, instead of predicting CLD development, prediction of different aspects of neonatal respiratory course may create new possibilities as to specific preventive measures in clinical practice as well as future research, for example, early selection of infants at high risk of postnatal corticosteroid therapy in order to study the effects of early administration of postnatal corticosteroid therapy.

\subsection{Importance of respiratory function in the early years after preterm birth}

Since severity of acute neonatal respiratory disease is decreasing and survival of extremely preterm infants is increasing, long-term consequences regarding respiratory function probably have changed considerably in ventilated preterm infants during past decades. The severe respiratory condition, as was described by Northway and colleagues in 1967, 188 is not frequently seen nowadays. Interruption of pulmonary development by preterm birth seems to be the main feature of neonatal respiratory disease nowadays, leading to abnormal postnatal lung growth and development and, eventually, hypoalveolarisation and decreased vascularisation. ${ }^{57}$ Although most children may not notice this during normal daily activities, a substantial number of infants will during exercise. According to respiratory function tests performed for the studies in this thesis, the majority of infants showed increased resistance, and decreased compliance of the respiratory system (Chapter 6). According to respiratory function tests performed at the age of two, the majority of infants showed increased bronchial reactivity (Chapter 7), indicating that various aspecific stimuli may cause increased airway resistance, elevating the work of breathing.

Studying associations between early clinical and mechanical patient characteristics and respiratory outcome during subsequent years may reveal important determinants for respiratory course during infancy. Results in Chapter $\mathbf{6}$ indicated that inborn properties of the respiratory system are important determinants of the need for inhalation therapy with bronchodilators or corticosteroids during the first year of life and respiratory function at one year of age, i.e. respiratory system resistance and 
compliance. Other factors, like degree of prematurity, expressed as gestational age or birth weight, severity of neonatal respiratory disease and neonatal treatment, like ventilator conditions and duration of ventilator therapy, were of minor importance. Only the number of days requiring supplemental oxygen was additionally associated with respiratory system compliance at one year of age. These findings resemble results from previous studies in healthy term infants, $48,166,167,232,274$ revealing that premorbid airway function was associated with the occurrence of wheezing and respiratory function in subsequent years. The fact that also in preterm ventilated infants, longterm respiratory sequelae seem to be mainly determined by inborn properties of the respiratory system and less by factors associated with prematurity, suggests that longterm respiratory course is mainly affected by intra-uterine pulmonary development. However, in Chapter 7 severity of neonatal lung damage, indicated by respiratory system compliance, RDS severity and inspiratory pressures, seems to be the main factor related to the development of bronchial hyperreactivity. These results are in line with previous findings regarding associations between prematurity ${ }^{79,94,195}$ or CLD 174,181,195,223 and subsequent development of bronchial hyperresponsiveness in childhood. When summarizing the main findings of Chapter 6 and Chapter 7, it appears that early neonatal determinants of long-term respiratory course may vary according to the outcome measure studied. Evidently, respiratory symptoms of coughing and wheezing during infancy predominantly depend on airway diameter, which is not the main part of the lungs being irreversibly damaged after prematurity. On the other hand, development of bronchial hyperreactivity during infancy seems to be mainly related to degree of neonatal parenchymal damage to the lungs, which can be irreversibly damaged by various events during the neonatal period.

These results suggest a certain degree of tracking of respiratory function throughout the first years of life in ventilated preterm infants, which was partially confirmed by the results in Chapter 8. Both respiratory system resistance and compliance improved considerably during the first year of life after preterm birth. Airway enlargement and extensive alveolarisation probably account for this. Although interindividual differences in respiratory function declined during the first year of life, tracking was partly present for mainly resistance of the respiratory system during the first year of life. Additionally, bronchial responsiveness at two years of age was consistently associated with compliance during the first year of life, but inconsistently with respiratory system resistance, supporting the findings reported in Chapter 7.

\subsection{Main conclusions}

The studies in this thesis show that in the current surfactant era, the majority of ventilated preterm infants are still suffering from respiratory morbidity and substantial respiratory function abnormalities throughout the early years of life. Since respiratory 
function testing during mechanical ventilation does have important limitations, certain preparatives have to be made before reliable results can be obtained and patients are not getting extra disturbed. Inborn resistive properties of the respiratory system are important in relation to early neonatal respiratory course as well as subsequent respiratory morbidity and respiratory function during the first year of life. With regard to the first-year respiratory course, inborn resistive properties are more important determinants than degree of prematurity and neonatal lung damage. However, in relation to the development of bronchial hyperresponsiveness during infancy, degree of neonatal parenchymal damage to the lung is most important. Tracking of respiratory function is partially present for the resistive properties of the respiratory system during the early years after birth in preterm ventilated infants.

\subsection{Future perspectives}

Despite the fact that the respiratory function technique used during mechanical ventilation in the studies of this thesis have been executed in research settings since the mid-1980s and recommendations of the European Respiratory Society are available to ensure a acceptable level of uniformity and reliability, methodological differences between studies may still be substantial. Further standardization of the technique is required and should focus on both practical application and correction for effects of changes in gas viscosity and other influencing factors, like endotracheal tube resistance.

The neonatal respiratory condition in ventilated preterm infants has changed considerably since the 1950 s, when mechanical ventilation of these infants was first applied. It is likely that together with this evolvement, early neonatal determinants of respiratory course have changed in these infants. Since the results in this thesis indicate that early respiratory function measurements provide useful information with respect to both the actual respiratory condition and neonatal respiratory course, further studies are needed to evaluate whether implementation of early respiratory function measurements in clinical practice will have consequences for neonatal care and respiratory outcome. Recent evidence regarding adverse long-term effects of postnatal dexamethason therapy, increases the need to search for alternative therapies with, for example, less potent steroids with less unwanted long-term side effects. Early selection of high-risk infants using our prediction model of postnatal corticosteroid therapy, helps to limit unnecessary exposure of these vulnerable infants to new treatment strategies. Early selection of infants at high risk of prolonged mechanical ventilation may create possibilities for early implementation of preventive measures. 
Instead of measures indicating degree of prematurity, inborn resistive properties of the respiratory system are the most important determinants of respiratory course during the first year of life. To decrease the risk of persistent respiratory morbidity and functional abnormalities, it is essential to reveal the determinants of early lung function. This can be achieved by a large prospective multicentre trial evaluating all pregnant women in a certain geographic area with regard to:

- familial predisposition

- $\quad$ way of conception (artificial, spontaneous)

- maternal smoking

- invasive procedures

- antenatal infections

- nutritional state

- antenatal corticosteroid therapy

- information by ultrasound about foetal growth and amniotic fluid

- inflammatory cytokines, nicotine, and IgE-level in maternal and foetal blood

By investigating associations between these data and early postnatal respiratory function and subsequent respiratory course, determinants of postnatal respiratory course can be revealed in both term and preterm infants. The results of such a trial may also increase the possibilities for prevention of persistent respiratory morbidity and functional abnormalities.

The degree of neonatal lung damage being important in the development of bronchial hyperresponsiveness during infancy in ventilated preterm infants emphasizes the need to further improve neonatal care. Hence, prospective clinical intervention trials investigating new strategies to decrease lung damage have to be conducted. For example, early postnatal administration of corticosteroids, using other, less potent, agents compared to dexamethasone at lower doses, may reduce the development of neonatal lung damage and subsequent bronchial hyperresponsiveness and may have less severe long-term consequences. 


\subsection{Inleiding}

In de studies, die beschreven worden in dit proefschrift, worden de mechanische eigenschappen van het ademhalingssysteem onderzocht tijdens de periode vlak na de geboorte en de eerste levensjaren bij te vroeg geboren kinderen (= prematuren), die kunstmatige beademing nodig hadden vanaf de eerste dag na de geboorte. De overleving van deze kinderen is sterk verbeterd sinds vanaf de 50 -er jaren kunstmatige beademing voor het eerst ook bij te vroeg geboren kinderen werd toegepast. Als gevolg hiervan werden artsen geconfronteerd met nieuwe ziektebeelden, die gerelateerd waren aan de onrijpheid van organen bij deze nieuwe patiëntenpopulatie. Problemen met en ziektebeelden van het ademhalingssysteem zijn veranderd door verbeteringen in de zorg voor pasgeborenen, zoals moderne beademingsapparatuur, die fijner afgesteld kan worden dan voorheen, en medicatie als corticosteroiden voor de geboorte aan moeder om de longrijping bij het kind te bevorderen en surfactant na de geboorte aan het kind om het openen van de longen te bevorderen. De resultaten van de studies in dit proefschrift geven ons een actueel beeld van klinische en mechanische factoren, die van belang zijn voor het beloop gedurende de eerste jaren na de geboorte bij beademde, prematuur geboren kinderen.

\subsection{Beperkingen van longfunctiemetingen bij beademde prematuren}

Bij beademde prematuren zijn de mogelijkheden voor longfunctiemetingen beperkt (Hoofdstuk 2). Dit komt met name door de verminderde lichamelijke conditie van deze kinderen en de specifieke omgeving op de intensive care afdeling voor pasgeborenen. Door de instabiele klinische toestand als gevolg van de onrijpheid van verschillende orgaansystemen bij deze kinderen, kunnen kleine gebeurtenissen of veranderingen reeds een serieuze verslechtering van hun lichamelijke conditie veroorzaken. Het is daarom van groot belang om het aantal momenten waarop deze patiënten gestoord worden zoveel mogelijk te beperken. Dat is de reden waarom longfunctiemetingen voor onderzoeksdoeleinden bij voorkeur meteen na een routine verzorgingsbeurt ingepland worden. Tijdens zo'n verzorgingsbeurt wordt ook de beademingsbuis uitgezogen om eventuele slijmophoping in de buis te verwijderen. Verder is het van belang dat tijdens de meting het kind geen extra inspanning hoeft te leveren om te ademen. Daarom moet de apparatuur die gebruikt wordt voor de longfunctiemetingen aan strenge eisen voldoen, waarbij de extra dode ruimte en weerstand minimaal zijn.

Naast de beperkingen die voortkomen uit een verminderde lichamelijke conditie bij beademde prematuren, worden longfunctiemetingen beperkt door specifieke omgevingsfactoren op de intensive care voor pasgeborenen. Vaak vergroten deze factoren het aandeel van ruissignalen, die opgevangen worden tijdens de metingen, of 
Algemene beschouwing en toekomstperspectief

zorgen ze er op een andere manier voor dat het gewenste signaal minder duidelijk wordt. Zo is algemeen bekend dat bij beademde kinderen de gemeten weerstand aanzienlijk verhoogd wordt door de aanwezigheid van de beademingsbuis in de luchtpijp. ${ }^{152}$ Correctie hiervoor is mogelijk door de weerstand van de beademingsbuis af te trekken van de gemeten weerstand. Hiervoor moet de weerstand van de beademingsbuis bepaald worden zonder dat deze ingebracht is in het kind (= in vitro). In eerdere studies is echter gebleken dat deze weerstand niet goed overeenkomt met de werkelijke weerstand van de beademingsbuis op het moment dat deze in de luchtpijp zit (= in vivo). ${ }^{152,164}$ Dat betekent dat correcties met in vitro bepaalde waarden niet bruikbaar zijn voor in vivo situaties. Daarom is de gemeten weerstand in de studies in dit proefschrift niet gecorrigeerd voor de weerstand van de beademingsbuis. De actuele longfunctie tijdens beademing wordt ook beïnvloed door de alineaire druk-volume relatie als gevolg van het dichtvallen van de longblaasjes aan het einde van de uitademing (= expiratie) en het overmatig uitzetten van een aantal longgedeelten aan het einde van de inademing (= inspiratie). Het feit dat de kinderen tijdens de longfunctiemetingen uitademen in een beademingscircuit, waarin een positieve eind-expiratoire druk (PEEP) gehandhaafd wordt, heeft ook een effect op de resultaten van de longfunctiemetingen. Echter, het opheffen van deze positieve druk heeft weer negatieve klinische gevolgen voor deze kwetsbare kinderen. Een ander belangrijk aspect is het effect van de physische samenstelling van de beademingsgassen op de metingen van luchtstroomsnelheid (flow) door flow-meters (pneumotachometers). Deze problematiek wordt belicht in Hoofdstuk 3. Eerdere studies hebben de effecten van relatieve luchtvochtigheid, temperatuur, en druk van

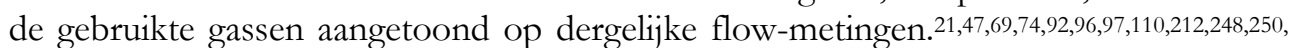
263,269 Ook kenmerken van de rubberen slangen, die verbonden zijn aan beademingsapparatuur, en de manier waarop zij aangesloten zijn hebben een effect op flow-metingen. ${ }^{142}$ De invloed van al deze effecten op de metingen zijn minimaal wanneer de omstandigheden tijdens de kalibratie-procedure, wanneer de apparatuur geijkt wordt, identiek zijn aan de omstandigheden tijdens de meting zelf of wanneer achteraf een juiste correctie wordt toegepast. De beademingsapparatuur zelf is ook afhankelijk van flow-metingen door een pneumotachometer. Hoewel in recent ontwikkelde beademingsmachines flow-registraties gecorrigeerd worden voor lichaamstemperatuur en druk bij volledige verzadiging met water (BTPS), vindt tot op heden nog geen correctie plaats voor het gebruikte zuurstofpercentage en de luchtstroomsnelheid tijdens beademing. In dit proefschrift worden correctiefactoren gegeven om te corrigeren voor deze effecten, waarbij specifieke omstandigheden gelden tijdens de kalibratie-procedure. Een andere beperkende factor voor longfunctiemetingen bij beademde prematuren is de afhankelijkheid van couveuse en beademingsapparatuur. Verder zijn deze kinderen niet in staat om actief mee te werken tijdens de meting of vervoerd te worden van de intensive care voor 
pasgeborenen naar de afdeling kinderlongziekten, waar gespecialiseerde apparatuur voor de metingen beschikbaar is.

\subsection{Het belang van longfunctiemetingen tijdens de eerste maanden na de geboorte bij beademde prematuren}

Om een idee te kriigen van de conditie van het ademhalingssysteem bij beademde prematuren wordt gebruik gemaakt van een aantal hulpmiddelen, zoals een röntgenfoto van de longen, bloedgasanalyse voor de partiële koolzuurspanning en zuurstofspanning in het bloed, pulse oximetrie voor een indirecte meting van de zuurstofsaturatie in het bloed, de beademingsvoorwaarden op dat moment en een continue registratie van de adembewegingen van het kind. Hulpmiddelen waarvan de interpretatie redelijk objectief is, zoals bloedgasanalyse, zijn vaak ingrijpend voor het kind. Hiervoor is namelijk bloedafname noodzakelijk. Röntgenfoto's kunnen eenvoudig gemaakt kunnen worden zonder dat de patiënt hier veel van merkt, maar de interpretatie ervan is subjectief, waardoor er aanzienlijke verschillen kunnen bestaan tussen verschillende beoordelaars. ${ }^{213}$ In een poging dit te verbeteren zijn er in het verleden verschillende scoringssystemen ontwikkeld, waarbij nauwkeurig omschreven aspecten van dergelijke röntgenfoto's worden gescoord volgens een bepaalde rangorde in ernst. Ondanks deze pogingen om de verschillen in beoordeling van röntgenfoto's te verkleinen, tonen de resultaten in Hoofdstuk 4 aan dat de scores van verschillende beoordelaars niet goed met elkaar overeenkomen. Daarnaast werd geen relatie gevonden tussen de röntgenscores en de longfunctie op dat moment. Dit kan verklaard worden door de verschillen in de beoordelingen, maar ook door het feit dat tijdens het meten van de longfunctie hele andere aspecten van de zieke long onderzocht worden dan tijdens röntgenonderzoek.

Naast het bepalen van de actuele conditie van het ademhalingssysteem, is het voorspellen van het individuele respiratoire beloop tijdens de eerste maanden na de geboorte van belang voor een optimale zorg bij beademde prematuren. Een hogere prioriteit voor preventieve maatregelen tijdens de behandeling van pasgeborenen met een verhoogd risico op problemen met het ademhalingssysteem zal waarschijnlijk het beloop bij deze kinderen verbeteren. In Hoofdstuk $\mathbf{5}$ worden betrouwbare modellen gepresenteerd om te kunnen voorspellen welke kinderen uiteindelijk corticosteroiden als medicatie nodig hebben en welke kinderen langdurige beademd zullen worden. Dit zijn twee belangrijke aspecten van het respiratoire beloop bij beademde prematuren. In eerdere studies zijn met name voorspellingsmodellen voor de ontwikkeling van chronische longziekte ${ }^{91}$ bij deze kinderen beschreven met als criterium extra zuurstofbehoefte na 28 dagen na de geboorte $19,62,89,160,210,215,256,270$ of bij 36 weken $^{87,95,160,214}$ na de conceptie. Slechts enkele hiervan konden toegepast worden binnen 48 uur na de geboorte, ${ }^{70,137,154}$ zoals bij onze studie. En aangezien deze laatste 
Algemene beschouning en toekomstperspectief

studies gedaan waren in de periode voordat er twee belangrijke ontwikkelingen plaatsvonden in de behandeling van beademde prematuren (namelijk: corticosteroiden voor de geboorte toedienen aan moeder om de longrijping bij het kind te bevorderen en surfactant na de geboorte toedienen aan het kind om het openen van de longen te bevorderen), zijn deze resultaten in het huidige tijdperk niet meer bruikbaar voor de klinische praktijk. Verder creëert het voorspellen van verschillende onderdelen van het respiratoire beloop bij deze kinderen, zoals corticosteroiden-toediening en langdurige beademing, in plaats van een algemene voorspelling van chronische longziekte nieuwe mogelijkheden. Preventieve maatregelen in de klinische praktijk kunnen op deze manier gerichter ingesteld worden. Maar ook voor verder onderzoek in de toekomst biedt dit voordelen, zoals bijvoorbeeld voor een vroege selectie van kinderen met een verhoogd risico op corticosteroiden-toediening om de effecten van toediening van deze medicatie in een veel vroegere fase te onderzoeken.

\subsection{Het belang van longfunctiemetingen in de eerste jaren na vroeggeboorte}

Gedurende de afgelopen decennia worden een aantal ontwikkelingen gezien op het gebied van longziekten bij beademde prematuren. De ernst van acute longziekte na de geboorte (RDS) is in deze periode afgenomen en de overleving van steeds vroeger geboren kinderen neemt toe. Dit heeft belangrijke consequenties voor de longfunctie op de lange termijn. Ernstige longziektebeelden, zoals die beschreven werden door Northway in 1967, 188 worden nog nauwelijks gezien op de dag van vandaag. Het ziektebeeld dat we op dit moment meestal zien wordt gekenmerkt door een onderbreking in de longontwikkeling door vroeggeboorte. Dit kan leiden tot een abnormale longgroei en -ontwikkeling vanaf de geboorte met als gevolg te weinig longblaasjes en een gestoorde doorbloeding. ${ }^{57}$ De meeste kinderen merken hier weinig van tijdens hun normale dagelijkse activiteiten, maar bij een toegenomen lichamelijke inspanning merken ze vaak wel dat ze tekort komen. Uit de longfunctiemetingen die voor de studies in dit proefschrift verricht zijn blijkt dat de meerderheid van de beademde prematuren een afwijkende longfunctie had met een verhoogde weerstand en een verlaagde compliantie (= toegenomen stijfheid) van het ademhalingssysteem op de leeftijd van 1 jaar (Hoofdstuk 6). Op tweejarige leeftijd bleek de gevoeligheid van de luchtwegen bij de meerderheid van de kinderen toegenomen te zijn (Hoofdstuk 7). Dit betekent dat verschillende aspecifieke factoren, zoals mist of een rokerige ruimte, ervoor kunnen zorgen dat de weerstand van de luchtwegen sterk toeneemt, waardoor kinderen veel meer moeite hebben met ademen.

Door vroege klinische en mechanische factoren van vlak na de geboorte bij deze kinderen te relateren aan het beloop gedurende de jaren erna, hopen we belangrijke determinanten te vinden voor dit beloop. De resultaten in Hoofdstuk 6 geven aan dat 
de kenmerken van het ademhalingssysteem, zoals ze aanwezig zijn bij de geboorte, belangrijke determinanten zijn voor het al dan niet nodig hebben van medicatie als luchtwegverwijders of ontstekingsremmers tijdens het eerste levensjaar. Ook blijken dit belangrijke determinanten te zijn voor de longfunctie op 1-jarige leeftijd. Andere factoren, zoals de mate van vroeggeboorte, uitgedrukt in zwangerschapsduur of geboortegewicht, de ernst van RDS en de behandeling die gegeven wordt, zoals beademingsvoorwaarden en beademingsduur, bleken van minder groot belang. Alleen het aantal dagen waarop extra zuurstoftoediening nodig was bleek tevens gerelateerd te zijn aan de compliantie van het ademhalingssysteem op 1-jarige leeftijd. Deze bevindingen komen overeen met resultaten van eerdere studies bij gezonde kinderen, die geboren waren na een normale zwangerschapsduur (= à term).48,166,167,232,274 In deze studies kwam naar voren dat de luchtwegfunctie vóór het doormaken van een luchtweginfectie gerelateerd was aan het optreden van klachten van een piepende ademhaling en/of hoesten en de longfunctie in de jaren erna. Het feit dat de bevindingen in onze studies bij beademde prematuren overeenkomen met de bevindingen bij gezonde kinderen, suggereert dat de lange termijn gevolgen voor het ademhalingssysteem met name bepaald worden door de longontwikkeling tot de geboorte en minder door factoren vlak na vroeggeboorte, zoals acute longziekte en beademing. Echter, in Hoofdstuk 7 blijkt dat de ernst van longziekte en de daardoor ontstane longschade wel een belangrijke determinant blijkt te zijn voor het ontwikkelen van overgevoeligheid van de luchtwegen. Deze resultaten komen overeen met eerdere bevindingen in de literatuur, waarin relaties worden beschreven tussen vroeggeboorte ${ }^{79,94,195}$ of de ontwikkeling van chronische longziekte ${ }^{174,181,195,223}$ en overgevoeligheid van de luchtwegen op de peuterleeftijd. Als we de resultaten van Hoofdstukken 6 en 7 samenvatten valt op dat vroege determinanten van het lange termijn beloop afhankelijk zijn van de uitkomstmaat die bestudeerd wordt. Klachten van hoesten en piepen op de jonge kinderleeftijd zijn voornamelijk gerelateerd aan de diameter van de luchtwegen. Dit gedeelte van het ademhalingssysteem is niet het belangrijkste gedeelte dat onherstelbaar beschadigd wordt na vroeggeboorte. Vandaar dat voor deze uitkomstmaten de aangeboren eigenschappen van het ademhalingssysteem belangrijker lijken dan factoren die met name verantwoordelijk zijn voor het ontstaan van longschade, zoals vroeggeboorte, RDS en beademing. Aan de andere kant lijkt de ontwikkeling van overgevoeligheid van de luchtwegen wel met name samen te hangen met de mate van longschade door deze factoren.

De longfunctieresultaten tijdens de eerste twee levensjaren bij beademde prematuren suggereren een zekere mate van tracking, zoals beschreven wordt in Hoofdstuk 8. Dat betekent dat de kinderen met de slechtste longfunctie vlak na de geboorte op 1- en 2-jarige leeftijd nog steeds de slechtste longfunctie hebben binnen deze studiepopulatie. Voor alle kinderen gold dat zowel de weerstand als de compliantie van het ademhalingssysteem sterk verbeterden tijdens het eerste jaar na vroeggeboorte. Dit wordt veroorzaakt door longgroei en -ontwikkeling, waarbij de 
Algemene beschouwing en toekomstperspectief

luchtwegdiameter toeneemt en het aantal longblaasjes explosief stijgt. Hoewel de verschillen tussen de kinderen gedurende deze periode kleiner werden, bleef er een zekere mate van tracking bestaan voor met name de weerstand. Daarnaast bleek de gevoeligheid van de luchtwegen op 2-jarige leeftijd gerelateerd te zijn aan het verloop van de compliantie tijdens het eerste levensjaar en niet aan het verloop van de weerstand tijdens dezelfde periode. Dit bevestigt de resultaten in Hoofdstuk 7.

\subsection{Belangrijkste conclusies}

De studies in dit proefschrift tonen aan dat tijdens het huidige tijdperk, waarin op uitgebreide schaal corticosteroiden voor de geboorte en surfactant na de geboorte worden toegepast, de meerderheid van de beademde prematuren klachten en longfunctie-afwijkingen houden tijdens de eerste levensjaren. Aangezien longfunctiemetingen tijdens de beademing belangrijke beperkingen kennen, moeten bepaalde maatregelen getroffen worden om betrouwbare resultaten te verkrijgen en te voorkomen dat deze kwetsbare patiënten onnodig gestoord worden.

Bepaalde aangeboren eigenschappen van het ademhalingssysteem, zoals de weerstand, blijken in belangrijke mate gerelateerd te zijn aan zowel het vroege klinische beloop als het hebben van klachten en longfunctieafwijkingen tijdens het eerste levensjaar. Met betrekking tot het respiratoire beloop tijdens het eerste jaar zijn deze eigenschappen belangrijker dan determinanten als vroeggeboorte, longziekte en beademing, die belangrijk zijn bij het ontstaan van onherstelbare longschade. Echter, met betrekking tot gevoeligheid van de luchtwegen op 2-jarige leeftijd lijken deze oorzakelijke factoren voor longschade wel belangrijke determinanten te zijn. Als laatste blijkt de longfunctie tijdens de eerste 2 jaar na vroeggeboorte een zekere mate van tracking te laten zien, waarbij de kinderen met de slechtste longfunctie aan het begin over het algemeen de slechtste longfunctie houden.

\subsection{Toekomstperspectief}

De techniek die gebruikt werd om de longfunctie te meten in de studies in dit proefschift wordt reeds vanaf halverwege de jaren 80 van de vorige eeuw toegepast voor onderzoeksdoeleinden. Er zijn ook Europese criteria van de European Respiratory Society beschikbaar om een acceptabel niveau van uniformiteit en betrouwbaarheid te waarborgen. Toch worden vaak nog grote methodologische verschillen gevonden tussen verschillende studies, wat het vergelijken van onderzoeksresultaten bemoeilijkt. Daarom is verdere standaardisatie van de methode wenselijk en zou het focus hierbij moeten liggen op de praktische toepassing ervan en 
correctie voor effecten van veranderingen in de eigenschappen van het gebruikte gas en voor de weerstand van de beademingsbuis.

De respiratoire conditie van beademde prematuren is sterk veranderd sinds de jaren 50 van de vorige eeuw, toen voor het eerst kunstmatige beademing bij deze kinderen werd toegepast. Het is aannemelijk dat tegelijkertijd ook de determinanten voor het respiratoire beloop bij deze kinderen veranderd zijn. De resultaten in dit proefschrift geven aan dat vroege longfunctiemetingen nuttige informatie geven over de actuele conditie van het ademhalingsysteem en het beloop op de langere termijn. Of implementatie in de klinische praktijk werkelijk zinvol is voor de directe zorg van deze kinderen en de uitkomst op de langere termijn zal in de toekomst verder onderzocht moeten worden. Door recente bevindingen met betrekking tot de nadelige lange termijn effecten van het toedienen van corticosteroiden na de geboorte is de noodzaak voor het zoeken naar alternatieve behandelingsmethoden met bijvoorbeeld minder sterke corticosteroiden met minder ongewenste bijwerkingen sterk toegenomen. Voor toekomstige studies op dit gebied kunnen onze voorspellingsmodellen gebruikt worden om alleen prematuren te selecteren met een verhoogd risico, zodat onnodige blootstelling aan nieuwe behandelingsmethoden met mogelijk ongewenste bijwerkingen zoveel mogelijk voorkomen wordt. Daarnaast kan een vroege selectie van prematuren met een verhoogd risico op langdurige beademing extra mogelijkheden creëren voor preventieve maatregelen.

In plaats van factoren die gerelateerd zijn aan de mate van vroeggeboorte, blijken aangeboren eigenschappen van de longen, zoals weerstand, belangrijke determinanten te zijn voor het respiratoire beloop tijdens het eerste levensjaar bij beademde prematuren. Om het risico op aanhoudende klachten en longfunctie-afwijkingen zoveel mogelijk in te perken, is verder onderzoek naar de determinanten van deze aangeboren eigenschappen van groot belang. Pas dan kunnen eventuele preventieve maatregelen genomen worden. Hiervoor is een grote, prospectieve studie in meerdere centra nodig, die alle zwangere vrouwen in een bepaald geografisch gebied onderzoekt op verschillende factoren zoals:

- familiaire aanleg

- conceptiemethode (natuurlijk of kunstmatig)

- roken door moeder

- bepaalde diagnostische ingrepen

- doormaken van infecties

- voedingstoestand

- corticosteroiden-toediening voor de geboorte

- informatie van het echografisch onderzoek met betrekking tot de groei van het ongeboren kind en de hoeveelheid vruchtwater

- aanwezigheid van bepaalde ontstekingsfactoren, nicotine en bepaalde antistoffen (IgE-antistoffen) in het bloed van moeder en kind. 


\section{Algemene beschouning en toekomstperspectief}

Door relaties tussen deze factoren en de longfunctie vroeg na de geboorte te bestuderen kunnen determinanten gevonden worden die bepalend zijn voor het respiratoire beloop bij zowel te vroeg geboren als à term geboren kinderen. Het is aannemelijk dat met deze gegevens de mogelijkheden voor het voorkomen van aanhoudende klachten en longfunctie-afwijkingen zullen toenemen.

Het feit dat overgevoeligheid van de luchtwegen op de jonge kinderleeftijd gerelateerd lijkt te zijn an de mate van de longschade die ontstaan is vlak na vroeggeboorte geeft aan dat toekomstige inzet nodig is op het verbeteren van de zorg van deze kinderen vlak na de geboorte. Daarvoor is verder onderzoek naar nieuwe strategieën om longschade zoveel mogelijk te voorkomen of te verminderen nodig. Hierbij kan gedacht worden aan het vroegtijdig toedienen van andere, minder potente corticosteroiden in lagere doseringen of andere beademingstechnieken. 


\section{References}

1. Inhaled nitric oxide for preterm infants with severe respiratory failure. Phase III study is currently recruiting patients. http:/ / clinicaltrials.gov:

2. Polgar G, Promadhat V, editors. Pulmonary function testing in children: techniques and standards. 1971; Saunders, Philadelphia.

3. Comroe JH, editors. Pulmonary and respiratory physiology, Parts I and II. 1976; Dowden, Hutchinson \& Ross, Stroudsburg, PA.

4. Moore KL, editors. The developing human; clinically oriented embryology. 1988; edition. W.B. Saunders Company, Philadelphia.

5. Ahuja A, Oh N, Chao W, Spragg RG, Smith RM. Inhibition of the human neutrophil respiratory burst by native and synthetic surfactant. Cell Mol Biol 1996; 14: 496-503.

6. Altman DG, Some common problems in medical research: Inter-rater agreement. In: Practical statistics for medical research; Altman DG, editor. 1991; edition first: 403-409.

7. Aoyagi T, Kishi M, Yamaguchi K, Watanabe S, Improvement of the earpiece oximeter. In: Abstracts of the Japanese Society of Medical Electronics and Biological Engineering 1974; 90-91.

8. Avery ME, Mead J. Surface properties in relation to atelectasis and hyaline membrane disease. Am J Dis Child 1959; 97: 517-523.

9. Avital A, Bar-Yishay E, Springer C, Godfrey S. Bronchial provocation tests in young children using tracheal auscultation. J Pediatr 1988; 112: 591-594.

10. Ballard PL, Ning Y, Polk D, Ikegami M, Jobe A. Glucocorticoid regulation of surfactant components in immature lambs. Am J Physiol 1997; 273: L1048-L1057.

11. Bancalari E, Pulmonary function testing and other diagnostic laboratory procedures. In: Neonatal pulmonary care; Thibeault DW, Gregory GA, editors, 1986.

12. Bancalari E. Epidemiology and risk factors for the 'new' bronchopulmonary dysplasia. Neo Reviews 2000; 1: e2-e5.

13. Bancalari E, Abdenour GE, Feller R, Gannon J. Bronchopulmonary dysplasia: clinical presentation. J Pediatr 1979; 95: 819-823.

14. Bancalari E, del Moral R. Bronchopulmonary dysplasia and surfactant. Biol Neonate 2001; 80: 7-13.

15. Baraldi E, Filipone M, Trevisanuto D, Zanardo V, Zacchello F. Pulmonary function until two years of life in infants with bronchopulmonary dysplasia. Am J Respir Crit Care Med 1997; 155: 149-155.

16. Barrington KJ, Finer NN, Inhaled nitric oxide for respiratory failure in preterm infants (Cochrane Review). 2002; Oxford, England: Update Software.

17. Bates JHT, Turner MJ, Lanteri CJ, Jonson B, Sly PD, Measurement of flow and volume. In: Infant respiratory function testing; Stocks J, Sly PD, Tepper RS, Morgan WJ, editors. 1996; 81-116.

18. Benoist MR, Brouard JJ, Rufin P, Delacourt C, Waernessyckle S, Scheinmann P. Ability of new lung function tests to assess methacholine-induced airway obstruction in infants. Pediatr Pulmonol 1994; 18: 308-316. 
19. Bhutani VK, Abbasi S. Relative likelihood of bronchopulmonary dysplasia based on pulmonary mechanics measured in preterm neonates during the first week of life. $J$ Pediatr 1992; 120: 605-613.

20. Bland JM, Altman DG. Statistical methods for assessing agreement between two methods of clinical measurement. Lancet 1986; February 8: 307-310.

21. Blumenfeld W, Turney SZ, Cowley RA. Mathematical model for flow in the heated Fleisch pneumotachometer. Med Biol Eng Comput 1972; Sept: 546-551.

22. Blystad W, Landing BH, Smith CA. Pulmonary hyaline membranes in newborn infants; statistical, morphological and experimental study of their nature, occurrence and significance. Pediatrics 1951; 8: 5-21.

23. Bocking AD, McMillen IC, Harding R, Thorburn GD. Effect of reduced uterine blood flow on fetal and maternal cortisol. J Dev Physiol 1986; 8: 237-245.

24. Boss JH, Graig JM. Reparative phenomena in lungs of neonates with hyaline membranes. Pediatrics 1962; 29: 890-898.

25. Brion LP, Primhak RA, Intravenous or enteral loop diuretics for preterm infants with (or developing) chronic lung disease (Cochrane Review). 2003; Oxford, England: Update Software.

26. Carlo WA, Beoglos A, Siner BS, Martin RJ. Neck and body position effects on pulmonary mechanics in infants. Pediatrics 1989; 84: 670-674.

27. Carlo WA, Martin RJ. Principles of neonatal assisted ventilation. Pediatr Clin North Am 1986; 33: 221-237.

28. Carson J. The elasticity of the lungs. Philos Trans R Soc Lond 1820; 111: 29-44.

29. Chan KN, Elliman A, Bryan E, Silverman M. Clinical significance of airway responsiveness in children of low birthweight. Pediatr Pulmonol 1989; 7: 251-258.

30. Chan KN, Wong YC, Silverman M. Relationship between infant lung mechanics and childhood lung function in children of very low birthweight. Pediatr Pulmonol 1990; 8: 74 81.

31. Charafeddine L, D'Angio CT, Phelps DL. Atypical chronic lung disease patterns in neonates. Pediatrics 1999; 103: 759-765.

32. Clark RH, Gerstmann DR, Jobe AH, Moffitt ST, Slutsky AS, Yoder BA. Lung injury in neonates: Causes, strategies for prevention, and long-term consequences. J Pediatr 2001; 139: 478-486.

33. Clements JA, Avery ME. Lung surfactant and neonatal respiratory distress syndrome. Am J Respir Crit Care Med 1998; 157: S59-S66.

34. Cockroft DW, Killian DN, Mellon JA, Hargreave FE. Bronchial reactivity to inhaled histamine: a method and clinical survey. Clin Allergy 1977; 7: 235-243.

35. Colin AA, Wohl MEB, Mead J, Ratjen FA, Glass G, Stark AR. Transition from dynamically maintained to relaxed end-expiratory volume in human infants. J Appl Physiol 1989; 67: 2107-2111.

36. Coons S, Guilleminault C. The development of sleep-wake patterns and non-rapid eye movement sleep stages during the first six months of life in normal infants. Pediatrics 1982; 69: 793-798.

37. Crowley P, Prophylactic corticosteroids for preterm birth (Cochrane Review). 2002; Oxford, England: Update Software. 
38. Crowther CA, Alfirevic Z, Haslam RR, Prenatal thyrotropin-releasing hormone for preterm birth (Cochrane Review). 2001; 2: Oxford, England: Update Software.

39. Curzi-Dascalova L. Physiological correlates of sleep development in premature and fullterm neonates. Neurophysiol Clin 1992; 22: 151-156.

40. Davies H, Kitchman R, Gordon I, Helms P. Regional ventilation in infancy. N Engl J Med 1985; 313: 1626-1628.

41. Davis GM, Stocks J, Gerhardt T, Abbasi S, Gappa M, Measurement of dynamic lung mechanics in infants. In: Infant Respiratory Function Testing; Stocks J, Sly PD, Tepper RS, Morgan WJ, editors. 1996; 259-281.

42. Davis PG, Henderson-Smart DJ, Intravenous dexamethasone for extubation of newborn infants (Cochrane Review). 2002; Oxford, England: Update Software.

43. de Winter JP, Egberts J, Merth IT, Peerdeman A, van Bel F, Bakker GC, Quanjer PhH. Biochemical lung maturity, static respiratory compliance and pulmonary gas transfer in intubated preterm infants with and without respiratory distress syndrome. Pediatr Pulmonol 1995; 20: 152-159.

44. de Winter JP, Merth IT, Brand R, Quanjer PhH. Functional residual capacity and static compliance during the first year in preterm infants treated with surfactant. Am J Perinat 2000; 17: 377-384.

45. de Winter JP, Merth IT, van Bel F, Egberts J, Brand R, Quanjer PhH. Changes of respiratory system mechanics in ventilated lungs of preterm infants with two different schedules of surfactant treatment. Pediatr Res 1994; 35: 541-549.

46. Desai NS, Cunningham MD, Boyer DJ, Gagel DM. Predisposing pulmonary dynamics from birth for infants with bronchopulmonary dysplasia. Clin Res 1983; 31: 908A.

47. Devabhaktuni VG, Torres A, Wilson S, Yeh MP. Effect of nitric oxide, perfluorocarbon, and heliox on minute volume measurement and ventilator volumes delivered. Crit Care Med 1999; 27: 1603-1607.

48. Dezateux C, Stocks J, Wade AM, Dundas I, Fletcher ME. Airway function at one year: association with premorbid airway function, wheezing, and maternal smoking. Thorax 2001; 56: 680-686.

49. Dik WA, Versnel MA, Naber BAE, Janssen DJ, van Kaam AHLC, Zimmermann LJI. Dexamethasone treatment does not inhibit fibroproliferation in chronic lung disease of prematurity. Eur Respir J 2003; 21: 842-847.

50. Dimitriou G, Greenough A, Kavvadia V, Shute M, Karani J. A radiographic method for assessing lung area in neonates. BrJ Radiol 1999; 72: 335-338.

51. Dimitriou G, Greenough A, Giffin FJ, Karani J. The appearance of 'early' chest radiographs and the response to surfactant replacement therapy. Br J Radiol 1995; 68: 1177-1180.

52. Donders FC. Bijdrage tot het mechanisme van ademhaling en bloedsomloop in de gezonde en zieke toestand. Nederland Lancet 1849; 5: 333.

53. Dreizzen E, Migdal M, Praud J-P, Magny J-F, Dehan M, Chambille B, Gaultier C. Passive compliance of total respiratory system in preterm newborn infants with respiratory distress syndrome. J Pediatr 1988; 112: 778-781.

54. Dunbar H, Kotecha S. Domicilary oxygen for infants with chronic lung disease of prematurity. Care Crit III 2000; 16: 90-93. 
55. Dutch Perinatal Registration Foundation. Stichting Perinatale Registratie Nederland. Bilthoven, The Netherlands, 2003.

56. Eber E, Zach MS. Long term sequelae of bronchopulmonary dysplasia (chronic lung disease of infancy). Thorax 2001; 56: 317-323.

57. Eber E, Zach MS, Neonatal chronic lung disease and its long-term consequences. In: European Respiratory Mon; Bush A, Carlsen K-H, Zach MS, editors. 2002; 99-115.

58. Eber E, Zach MS, Neonatal chronic lung disease and its long-term consequences. In: European Respiratory Monograph; Bush A, Carlsen K-H, Zach MS, editors. 2002; 99-115.

59. Egreteau L, Pauchard J-Y, Semama DS, Matis J, Liska A, Romeo B, Cneude F, Hamon I, Truffert P. Chronic oxygen dependency in infants born at less than 32 weeks' gestation: incidence and risk factors. Pediatrics 2001; 108: E26.

60. Ehrenkranz RA, Walsh-Sukys MC, Vohr BR. New consensus definition of bronchopulmonary dysplasia (BPD-DEF) predicts pulmonary and neuro-developemental outcomes in early infancy. Pediatr Res 2001; 49: 276A.

61. European Respiratory Society/American Respiratory Society. Respiratory mechanics in infants: Physiologic evaluation in health and disease. Eur Respir J 1993; 6: 279-230.

62. Farstad T, Bratlid D. Incidence and prediction of bronchopulmonary dysplasia in a cohort of premature infants. Acta Paediatr 1994; 83: 19-24.

63. Fedrick J, Butler NR. Hyaline membrane disease. Lancet 1972; 2: 768-769.

64. Field D. Trent neonatal survey 1999 annual report. University of Leicester 2000.

65. Field D, Milner AD, Hopkin IE. Inspiratory time and tidal volume during intermittent positive pressure ventilation. Arch Dis Child 1985; 60: 259-261.

66. Finlay-Jones JM, Papadimitriou JM, Barter RA. Pulmonary hyaline membrane: Light and electron microscopic study of the early stage. J Pathol 1974; 112: 117.

67. Finucane KE, Egan BA, Dawson SV. Linearity and frequency response of pneumotachographs. J Appl Physiol 1972; 32: 121-126.

68. Fletcher ME, Baraldi E, Steinbrugger B, Passive Respiratory Mechanics. In: Infant Respiratory Function Testing; Stocks J, Sly PD, Tepper RS, Morgan WJ, editors. 1996; 283327.

69. Foitzik B, Schmalisch G, Wauer RR. Einfluss der physikalischen Eigenschaften des Atemgases auf die pneumotachographische Ventilationsmessung bei Neugeborenen. Biomed Tech 1994; 39(4): 85-92.

70. Freezer NJ, Sly D. Predictive value of measurements of respiratory mechanics in preterm infants with HMD. Pediatr Pulmonol 1993; 16: 116-123.

71. French N, Hagan R, Evans S, Godfrey M, Benninger H, Mullan A. Repeated antenatal corticosteroids: growth and early childhood outcome. Proc Perinatal Soc Aust New Zealand 1997; A12.

72. Frey U, Makkonen K, Wellman T, Beardsmore C, Silverman M. Alterations in airway wall properties in infants with a history of wheezing disorders. Am J Respir Crit Care Med 2000; 161: 1825-1829.

73. Frey U, Stocks J, Coates A, Sly P, Bates J. Specifications for equipment used for infant pulmonary function testing. Eur Respir J 2000; 16: 731-740.

74. Frey U, Stocks J, Sly P, Bates J. Specifications for signal processing and data handling used for infant pulmonary function testing. Eur Respir J 2000; 16: 1016-1022. 
75. Froese AB, McCullough PR, Sugiura M, Vaclavik S, Possmayer F, Moller F. Optimizing alveolar expansion prolongs the effectiveness of exogenous surfactant therapy in the adult rabbit. Am Rev Respir Dis 1993; 148: 569-577.

76. Fujiwara T, Konishi M, Chida S, Okuyama K, Ogawa Y, Takeuchi Y, Nishida H, Kito H, Fujimara M, Nakamura H. Surfactant replacement therapy with a single postventilatory dose of a reconstituted bovine surfactant in preterm neonates with respiratory distress syndrome: final analysis of a multicenter, double-blind, randomized trial and comparison with similar trials. The Surfactant-TA Study Group. Pediatrics 1990; 86: 753-764.

77. Fujiwara T, Maeta H, Chida S, Morita T, Watabe Y, Abe T. Artificial surfactant therapy in hyaline membrane disease. Lancet 1980; 1: 55-59.

78. Galdès-Sebaldt M, Sheller JR, Grogaard J, Stahlman M. Prematurity is associated with abnormal airway function in childhood. Pediatr Pulmonol 1989; 7: 259-264.

79. Galdès-Sebaldt M, Sheller JR, Grogaard J, Stahlman M. Prematurity is associated with abnormal airway function in childhood. Pediatr Pulmonol 1989; 7: 259-264.

80. Gappa M, Colin AA, Goetz I, Stocks J. Passive respiratory mechanics: the occlusion techniques. Eur Respir J 2001; 17: 141-148.

81. Gappa M, Rabbette PS, Costeloe KL, Stocks J. Assessment of passive respiratory compliance in healthy preterm infants: a critical evaluation. Pediatr Pulmonol 1993; 15: 304 311.

82. Gaultier C, Fletcher ME, Beardsmore C, Motoyama E, Stocks J, Measurement conditions. In: Infant respiratory function testing; Stocks J, Sly PD, Tepper RS, Morgan WJ, editors. 1996; 29-44.

83. Gaultier CI. Lung volumes in neonates and infants. Eur Respir J 1989; 2: 130s-134s.

84. Gerhardt T, Bancalari E. Chestwall compliance in full-term and premature infants. Acta Paediatr Scand 1980; 69: 359-364.

85. Gerhardt T, Hehre D, Feller R, Reifenberg L, Bancalari E. Serial determination of pulmonary function in infants with chronic lung disease. J Pediatr 1987; 110: 448-456.

86. Giedion A, Haefliger H, Dangel P. Acute pulmonary X-ray changes in hyaline membrane disease treated with artificial ventilation and positive end-expiratory pressure (PEP). Pediatr Radiol 1973; 3: 145-152.

87. Giffin F, Greenough A, Karani J. Chest radiograph appearance at $24 \mathrm{~h}$ of age -prediction of chronic oxygen dependency. Br J Radiol 1995; 68: 248-251.

88. Gitto E, Reiter RJ, Karbownik M, Xian-Tan D, Barberi I. Respiratory distress syndrome in the newborn: role of oxidative stress. Intensive Care Med 2001; 27: 1116-1123.

89. Goldman SL, Gerhardt T, Sonni R, Feller R, Hehre D, Tapia JL, Bancalari E. Early prediction of chronic lung disease by pulmonary function testing. J Pediatr 1983; 102: 613617.

90. Gonzales A, Sosenko IRS, Chandar J, Hummler H, Claure N, Bancalari E. Influence of infection on patent ductus arteriosus and chronic lung disease in premature infants weighing 1,000 grams or less. J Pediatr 1996; 128: 470-478.

91. Graff MA, Novo RP, Diaz M, Smith C, Hiatt M, Hegyi T. Compliance measurement in respiratory distress syndrome: the prediction of outcome. Pediatr Pulmonol 1986; 2: 332 336.

92. Green M. The effects of changes in gas viscosity on measurement of gas flow rates and volumes by the pneumotachograph. Physiol Soc 1965; 19P-20P. 
93. Greenough A. Promotion of respiratory health in utero and during infancy. Monaldi Arch Chest Dis 2000; 55: 251-255.

94. Greenough A, Giffin FJ, Yüksel B. Respiratory morbidity in preschool children born prematurely. Relationship to adverse neonatal events. Acta Paediatr 1996; 85: 772-777.

95. Greenough A, Kavvadia V, Johnson AH, Calvert S, Peacock J, Karani J. A simple chest radiograph score to predict chronic lung disease in prematurely born infants. Br J Radiol 1999; 72: 530-533.

96. Grenvik $\AA$, Hedstrand U. The reliability of pneumotachography in respirator ventilation. Acta Anaesthesiol Scand 1966; 10: 157-167.

97. Grenvik Å, Hedstrand U, Sjögren H. Problems in pneumotachography. Acta Anaesthesiol Scand 1966; 10: 147-155.

98. Guirau LMB, Solé D, Naspitz CK. Bronchoprovocation with methacholine in children under two years old: a follow-up study. I Invest Allergol Clin Immunol 1997; 7: 110-114.

99. Gutkowski P, Migdal M. Predicted values of pulmonary mechanics parameters in children under 2 years. Pneumonol Pol 1987; LV: 65-70.

100. Halliday HL, Ehrenkranz RA, Delayed ( $>3$ weeks) postnatal corticosteroids for chronic lung disease in preterm infants (Cochrane Review). 2001; 2: Update Software Ltd, Oxford, England: Update Software.

101. Halliday HL, Ehrenkranz RA, Early postnatal ( $<96$ hours) corticosteroids for preventing chronic lung disease in preterm infants (Cochrane Review). 2001; 2: Oxford, England: Update Software.

102. Halliday HL, Ehrenkranz RA, Moderately early (7-14 days) postnatal corticosteroids for preventing chronic lung disease in preterm infants (Cochrane Review). 2001; 2: Oxford, England: Update Software.

103. Hallman M, Glumoff V, Rämet M. Surfactant in respiratory distress syndrome and lung injury. Comp Biochem Physiol Part A 2001; 129: 287-294.

104. Hanley JA, McNeil BJ. The meaning and use of the area under a receiver operating characteristic (ROC) curve. Radiology 1982; 143: 29-36.

105. Hansen T, Corbet A, Chronic lung disease. In: Avery's diseases of the newborn; Taeusch HW, Ballard RA, editors. 1998; edition 7: 634-647.

106. Hansen T, Corbet A, Disorders of the transition. In: Avery's diseases of the newborn; Taeusch HW, Ballard RA, editors. 1998; edition 7: 602-629.

107. Hansen T, Corbet A, Lung development and function. In: Avery's diseases of the newborn; Taeusch HW, Ballard RA, editors. 1998; edition 7: 541-551.

108. Harding R, Cock ML, Louey S, Joyce BJ, Davey MG, Albuquerque CA, Hooper SB, Maritz GS. The compromised intra-uterine environment: implications for future lung health. Clin Exp Pharmacol Physiol 2000; 27: 965-974.

109. Harding R, Tester ML, Moss TJ, Davey MG, Louey S, Joyce B, Hooper SB, Maritz G. Effects of intra-uterine growth restriction on the control of breathing and lung development after birth. Clin Exp Pharmacol Physiol 2000; 27: 114-119.

110. Hardt von der H, Zywietz C. Reliability in pneumotachographic measurements. Respiration 1976; 33: 416-424.

111. Harrell FE, Lee KL, Mark DB. Multivariable prognostic models: issues in developing models, evaluating assumptions and adequacy, and measuring and reducing errors. Stat Med 1996; 15: 361-387. 
112. Hentschel R, Brune T, Franke N, Harms E, Jorch G. Sequential changes in compliance and resistance after bolus administration or slow infusion of surfactant in preterm infants. Intensive Care Med 2002; 28: 622-628.

113. Herting E, Sun B, Jarstrand C, Curstedt T, Robertson B. Surfactant improves lung function and mitigates bacterial growth in immature ventilated rabbits with experimentally induced neonatal group B streptococcal pneumonia. Arch Dis Child 1997; 76: F3-F8.

114. Hislop AA, Haworth SG. Airway size and structure in the normal fetal and infant lung and the effect of premature delivery and artificial ventilation. Am Rev Respir Dis 1989; 140: 1717-1726.

115. Hjalmarson O, Sandberg K. Abnormal lung function in healthy preterm infants. Am J Respir Crit Care Med 2002; 165: 83-87.

116. Hochheim K. Über einige Befunde in den Lungen von Neugeborenen und die Beziehung derselben zur Aspiration von Fruchtwasser. Centralbl Pathol 1903; 14: 537-538.

117. Hofhuis W, Huysman MWA, van der Wiel EC, Holland WPJ, Hop WCJ, Brinkhorst G, de Jongste JC, Merkus PJFM. Worsening of V'maxFRC in infants with chronic lung disease in the first year of life. Am J Respir Crit Care Med 2002; 166: 1539-1543.

118. Hoo A-F, Henschen M, Dezateux C, Costeloe K, Stocks J. Respiratory function among preterm infants whose mothers smoked during pregnancy. Am J Respir Crit Care Med 1998; 158: 700-705.

119. Hoo AF, Dezateux C, Henschen M, Costeloe K, Stocks J. Development of airway function in infancy after preterm delivery. J Pediatr 2002; 141: 652-658.

120. Horbar JD, Wright L, Soll R. A multicenter randomized trial comparing two surfactants for the treatment of neonatal RDS. Pediatrics 1993; 123: 757-765.

121. Hutchinson J. On the capacity of the lungs, and on the respiratory functions, with a view of establishing a precise and easy method of detecting disease by the spirometer. Med Chir Soc (Lond) Trans 1846; 29: 137-145, 170, 184, 219-220, 234, 247-252.

122. Inselman LS, Mellins RB. Growth and development of the lung. J Pediatr 1981; 98: 1-15.

123. Iscoe S, Department of Physiology QUKO. Respiratory physiology. Lecture Notes available at: http://meds.queensu.ca/medicine/physiol/undergrad/210/210resp/Resp_notes.pdf 2001.

124. Isdale JM, Thomson PD. The value of radiography in the initial grading of the idiopathic respiratory distress syndrome. S Afr med J 1979; 56: 707-710.

125. Jobe AH. An unknown Lung growth and development after very preterm birth. Am J Respir Crit Care Med 166: 1529-1530.

126. Jobe AH. Pulmonary surfactant therapy. N Engl J Med 1993; 328: 861-868.

127. Jobe AH. The new BPD: an arrest of lung development. Pediatr Res 1999; 46: 641-643.

128. Jobe AH. Glucocorticoids, inflammation and the perinatal lung. Semin Neonatol 2001; 6: $331-342$.

129. Jobe AH, Ikegami M. Mechanisms initiating lung injury in the preterm. Early Hum Dev 1998; 53: 81-94.

130. Jobe AH, Ikegami M. Lung development and function in preterm infants in the surfactant treatment era. Annu Rev Physiol 2000; 62: 825-846.

131. Jones CA, Cayabyab RG, Kwong KYC, Stotts C, Wong B, Hamdan A, Minoo P, deLemos RA. Undetectable interleukin (IL)-10 and persistent IL-8 expression early in 
hyaline membrane disease: a possible development basis for the predisposition to chronic lung inflammation in preterm newborns. Pediatr Res 1996; 39: 966-975.

132. Jonson B, Richard J-C, Straus C, Mancebo J, Lemaire F, Brochard L. Pressure-volume curves and compliance in acute lung injury: evidence of recruitment above the lower inflection point. Am J Respir Crit Care Med 1999; 159: 1172-1178.

133. Die Foetuslunge im gebornen Kinde 1835; Gebhardt Grimma.

134. Karlberg J. A biologically-oriented mathematical model (ICP) for human growth. Acta Paediatr Suppl 1989; 350: 70-94.

135. Kauffman SL. Acceleration of canalicular development in lungs of fetal mice exposed transplacentally to dexamethasone. Lab Invest 1977; 36: 395-401.

136. Kavvadia V, Greenough A, Itakura Y, Dimitriou G. Neonatal lung function in very immature infants with and without RDS. J Perinat Med 1999; 27: 382-387.

137. Kirpalani H, Schmidt B, Gaston S, Santos R, Wilkie R. Birthweight, early passive respiratory system mechanics, and ventilator requirements as predictors of outcome in premature infants with respiratory failure. Pediatr Pulmonol 1991; 10: 195-198.

138. Kliegman RM, Respiratory tract disorders. In: Nelson Textbook of Pediatrics; Behrman RE, Kliegman RM, Arvin AM, Nelson WE, editors, 1996.

139. Kosch PC, Stark AR. Dynamic maintenance of end-expiratory lung volume in full-term infants. J Appl Physiol 1987; 45: 18-23.

140. Kraemer R. Assessment of functional abnormalities in infants and children with lung disease. Agents Actions: Swiss J Pharmacol 1993; 40: 41-55.

141. Kraybill EN, Runyan DK, Bose CL, Khan JH. Risk factors for chronic lung disease in infants with birth weights of 751 to 1000 grams. J Pediatr 1989; 115: 115-120.

142. Kreit JW, Sciurba FC. The accuracy of pneumotachograph measurements during mechanical ventilation. Am J Crit Care 1996; 154: 913-917.

143. Krieger I. Mechanics of respiration in bronchiolitis. Pediatrics 1964; 33: 45-54.

144. Kuehni CE, Davis A, Brooke AM, Silverman M. Are all wheezing disorders in very young (preschool) children increasing in prevalence? Lancet 2001; 357: 1821-1825.

145. Lal MK, Manktelow BN, Draper ES, Field DJ. Chronic lung disease of prematurity and intrauterine growth retardation: a population-based study. Pediatrics 2003; 111: 483-487.

146. Langston C, Kida K, Reed M, Thurlbeck WM. Human lung growth in late gestation and in the neonate. Am Rev Respir Dis 1984; 129: 607-613.

147. Lankenau HM. A genetic and statistical study of the respiratory distress syndrome. Eur J Pediatr 1976; 123: 167-177.

148. Le Souef PN. Validity of methods used to test airway responsiveness in children. Lancet 1992; 339: 1282-1284.

149. Le Souëf PN. Can measurements of airway responsiveness be standardized in children? Eur Respir J 1993; 6: 1085-1087.

150. Le Souef PN, England SJ, Bryan AC. Passive respiratory mechanics in newborns and children. Am Rev Respir Dis 1984; 129: 552-556.

151. Lebourges F, Moriette G, Boulé M, Delaperche MF, Relier JP, Gaultier C. Pulmonary function in infancy and childhood following mechanical ventilation in the neonatal period. Pediatr Pulmonol 1990; 9: 34-40.

152. LeSouef PN, England SJ, Bryan AC. Total resistance of the respiratory system in preterm infants with and without an endotracheal tube. J Pediatr 1984; 104: 108-111. 
153. Lindroth M, Mortensson W. Long-term follow-up of ventilator treated low birthweight infants. Acta Paediatr Scand 1986; 75: 819-826.

154. Lischka A, Coradello H, Simbruner G, Popow Ch, Pollak A. Comparison of chest radiography and static respiratory compliance in the assessment of the severity of pulmonary diseases in newborns with respiratory distress. Pediatr Radiol 1984; 14: 369_ 372.

155. Lister P, Iles R, Shaw B, Ducharme F, Inhaled steroids for neonatal chronic lung disease (Cochrane Review). 2002; Oxford, England: Update Software.

156. Little SA, Elkholy MM, Chalmers GW, Farouk A, Patel KR, Thomson NC. Predictors of nocturnal oxygen desaturation in patients with COPD. Respir Med 1999; 93: 202-207.

157. Lodrup Carlsen KC, Jaakkola JJK, Nafstad P, Carlsen K-H. In utero exposure to cigarette smoking influences lung function at birth. Eur Respir J 1997; 10: 1774-1779.

158. Lopuhaä CE, Roseboom TJ, Osmond C, Barker DJP, Ravelli ACJ, Bleker OP, van der Zee JS, van der Meulen HP. Atopy, lung function, and obstructive airways disease after prenatal exposure to famine. Thorax 2000; 55: 555-561.

159. Loughlin GM, Carroll JL, Sleep and respiratory disease in children. In: Principles and practice of sleep medicine in the child; Ferber R, Kryger M, editors. 1995; 217-230.

160. Lui K, Lloyd J, Ang E, Rynn M, Gupta JM. Early changes in respiratory compliance and resistance during the development of bronchopulmonary dysplasia in the era of surfactant therapy. Pediatr Pulmonol 2000; 30: 282-290.

161. Lyon A. Chronic lung disease of prematurity. The role of intra-uterine infection. Eur J Pediatr 2000; 159: 798-802.

162. Magraf LR, Tomashefski JF, Bruce MC, Dahms BB. Morphometric analysis of the lung in bronchopulmonary dysplasia. Am Rev Respir Dis 1991; 143: 391-400.

163. Mallol J, Willet K, Burton P, Sly PD. Influence of duration of occlusion time on respiratory mechanics measured with the single-breath technique in infants. Pediatr Pulmonol 1994; 17: 250-257.

164. Manczur T, Greenough A, Nicholson GP, Rafferty GF. Resistance of pediatric and neonatal endotracheal tubes: Influence of flow rate, size, and shape. Crit Care Med 2000; 28: 1595-1598.

165. Manktelow BN, Draper ES, Annamalai S, Field D. Factors affecting the incidence of chronic lung disease of prematurity in 1987, 1992, and 1997. Arch Dis Child Fetal Neonatal Ed 2001; 85: F33-F35.

166. Martinez FD, Morgan WJ, Wright AL, Holberg CJ, Taussig LM. Initial airway function is a risk factor for recurrent wheezing respiratory illnesses during the first three years of life. Am Rev Resp Dis 1991; 143: 312-316.

167. Martinez FD, Morgan WJ, Wright AL, Holberg CJ, Taussig LM, The Group Health Medical Associates. Diminished lung function as a predisposing factor for wheezing respiratory illness in infants. N Engl J Med 1988; 319: 1112-1117.

168. Massaro DJ, Massaro GD, The regulation of the formation of pulmonary alveoli. In: Chronic lung disease in early infancy; Bland RD, Coalson JJ, editors. 2000; 479-492.

169. Matthew DJ, Warner JO, Chrispin AR, Norman AP. The relationship between chest radiographic scores and respiratory function tests in children with cystic fibrosis. Pediatr Radiol 1977; 5: 198-200. 
170. Mead J. Measurement of inertia of the lungs at increased ambient pressure. J Appl Physiol 1956; 9: 208-212.

171. Mead J. Mechanical properties of the lungs. Physiol Rev 1961; 41: 281-330.

172. Merth IT, Quanjer PhH, Verschragen GJ, Functional residual capacity by the helium dilution method. In: A manual of infant lung function testing; Dezateux CA, Fletcher ME, Rabbette PS, Stanger LJ, Stocks J, editors. 1991.

173. Miller HC, Jennison MH. Study of pulmonary hyaline-like material in 4117 consecutive births. Incidence, pathogenesis, diagnosis. Pediatrics 1950; 5: 7-20.

174. Mochizuki H, Ohki Y, Nako Y, Morikawa A. Transcutaneous oxygen tension measurements during methacholine challenge of prematurity in infants with chronic lung disease. Pediatr Pulmonol 1998; 25: 338-342.

175. Mochizuki H, Ohki Y, Nako Y, Morikawa A. Bronchial reactivity to inhaled methacholine in infants with asthma and age-matched controls. J Asthma 1999; 36: 503-509.

176. Moessinger AC, Harding R, Adamson TM, Singh M, Kiu GT. Role of lung fluid volume in growth and maturation of the fetal sheep lung. J Clin Invest 1990; 86: 1270-1277.

177. Mohsenin V, Guffanti EE, Hilbert J, Ferranti R. Daytime oxygen saturation does not predict nocturnal oxygen desaturation in patients with chronic obstructive pulmonary disease. Arch Phys Med Rehabil 1994; 75: 285-288.

178. Montgomery GL, Tepper RS. Changes in airway reactivity with age in normal infants and young children. Am Rev Respir Dis 1990; 142: 1372-1376.

179. Moriette G, Gaudebout C, Clement A, Boule M, Gaultier C, Relier JP, Gaultier C. Pulmonary function at 1 year of age in survivors of neonatal respiratory distress: a multivariate analysis of factors associated with sequelae. Pediatr Pulmonol 1987; 3: 242-250.

180. Mortola JP, Fisher JT, Smith B, Fox G, Weeks S. Dynamics of breathing in infants. J Appl Physiol 1982; 52: 1209-1215.

181. Motoyama EK, Fort MD, Klesh KW, Mutich RL, Guthrie RD. Early onset of airway reactivity in premature infants with bronchopulmonary dysplasia. Am Rev Respir Dis 1987; 136: 50-57.

182. Muller NL, Bryan AC. Chest wall mechanics and respiratory muscles in infants. Pediatr Clin North Am 1979; 26: 503-516.

183. Naeye RL, Freeman RK, Blanc WA. Nutrition, sex, and fetal lung maturation. Pediatr Res 1974; 8: 200-204.

184. Neergard KV, Wirz K. Über eine Methode zur Messung der Lungenelastizität am lebenden Menschen insbesondere beim Emphysem. Z Klin Med 1927; 105: 51-82.

185. Nievas FF, Chernick V. Bronchopulmonary dysplasia (chronic lung disease of infancy) An update for the pediatrician. Clin Pediatr 2002; 41: 77-85.

186. Northway WA. Bronchopulmonary dysplasia: then and now. Arch Dis Child 1990; 65: 1076-1081.

187. Northway WH. Bronchopulmonary dysplasia: twenty-five years later. Pediatrics 1992; 89: 969-973.

188. Northway WH, Rosan RC, Porter DY. Pulmonary disease following respirator therapy of hyaline membrane disease: bronchopulmonary dysplasia. N Engl J Med 1967; 267: 357368.

189. Ogden BE, Murphy S, Saunders GC, Johnson JD. Lung lavage of newborns with respiratory distress syndrome. Chest 1983; 83S: 31-33. 
190. Olinsky A, Bryan AC, Bryan MH. A simple method of measuring total respiratory system compliance in newborn infants. S Afr Med J 1976; 50: 128-130.

191. Openshaw P, Edwards S, Helms P. Changes in rib cage geometry during childhood. Thorax 1984; 39: 624-627.

192. Pandya HC, Kotecha S. Chronic lung disease of prematurity: clinical and pathophysiological correlates. Monaldi Arch Chest Dis 2001; 56: 270-275.

193. Parat S, Delaperche M-F, Escourrou P, Denjean A, Gaulties C. Long-term pulmonary functional outcome of bronchopulmonary dysplasia and premature birth. Pediatr Pulmonol 1995; 20: 289-296.

194. Parker RA, Lindstrom DP, Cotton RB. Improved survival accounts for most, but not all, of the increase in bronchopulmonary dysplasia. Pediatrics 1992; 90 (5): 663-668.

195. Pelkonen AS, Hakulinen AL, Turpeinen M. Bronchial lability and responsiveness in school children born very preterm. Am J Respir Crit Care Med 1997; 158: 1178-1184.

196. Pelkonen AS, Hakulinen AL, Turpeinen M, Hallman M. Effect of neonatal surfactant therapy on lung function at school age in children born very preterm. Pediatr Pulmonol 1998; 25: 182-190.

197. Peters MJ, Tasker RC, Kiff KM, Yates R, Hatch DJ. Acute hypoxemic respiratory failure in children: case mix and the utility of respiratory severity indices. Intens Care Med 2000; 24: 699-705.

198. Phelan PD, Gracey M, Williams HE, Anderson EM. Ventilatory function in infants with cystic fibrosis. Arch Dis Child 1969; 44: 393-400.

199. Pillow JJ, Hall GL, Willet KE, Jobe AH, Hantos Z, Sly PD. Effects of gestation and antenatal steroid on airway and tissue mechanics in newborn lambs. Am J Respir Crit Care Med 2001; 163: 1158-1163.

200. Poets CF, Martin RJ, Noninvasive determination of blood gases. In: Infant respiratory function testing; Stocks J, Sly P, Tepper RS, Morgan WJ, editors. 1996; 411-443.

201. Quanjer PhH. Standardized lung function testing. Report Working Party Standardization of Lung Function Tests, European Community for Coal and Steel. Bull Eur Physiopathol Respir 1983; 19: 1-95.

202. Reid LM. Lung growth in health and disease. Br J Dis Chest 1984; 78: 113-134.

203. Reilly BJ, Featherby EA, Weng T-R, Crozier DN, Duic A, Levison H. The correlation of radiological changes with pulmonary function in cystic fibrosis. Radiology 1971; 98: 281285.

204. Rimensberger PC. Neonatal respiratory failure. Curr Opin Pediatr 2002; 14: 315-321.

205. Robert MF, Neff RK, Hubbell JP. Association between maternal diabetes and the respiratory distress syndrome in the newborn. N Engl J Med 1976; 294: 357-360.

206. Robertson B, Tunell R, Rudhe U. Late stages of pulmonary hyaline membranes of newborn. Acta Paediatr 1964; 53: 433-446.

207. Rohrer F. Der Strömungswiderstand in den menschlichen Atemwegen und der Einfluss der unregelmässigen Verzweigung des Bronchialsystems auf den Atmungsverlauf in verschiedenen Lungenbezirken. Pflügers Arch Gesamte Physiol Menschen Tiere 1915; 162: 225299.

208. Rohrer F. Der Zusammenhang der Atemkrafte und ihre Abhängigkeit von Dehnungszustand der Atemorgane. Pflügers Arch Gesamte Physiol Menschen Tiere 1916; 165: 419-444. 
209. Rojas MA, Gonzalez A, Bancalari E, Claure N, Poole C, Silva-Neto G. Changing trends in the epidemiology and pathogenesis of neonatal chronic lung disease. J Pediatr 1995; 126: 605-610.

210. Romagnoli C, Zecca E, Tortorolo L, Vento G, Tortorolo G. A scoring system to predict the evolution of respiratory distress syndrome into chronic lung disease in preterm infants. Intens Care Med 1998; 24: 476-480.

211. Rosenthal M, Bush A, The growing lung: normal development, and the long-term effects of pre- and postnatal insults. In: Eur Respir Mon; 2002; 19: 1-24. European Respiratory Society Journals Ltd., Sheffield, UK.

212. Roske K, Foitzik B, Wauer RR, Schmalisch G. Accuracy of volume measurements in mechanically ventilated newborns: a comparative study of commercial devices. J Clin Monit Comput 1998; 14: 413-420.

213. Rubenfeld GD, Caldwell E, Granton J, Hudson LD, Matthay MA. Interobserver Variability in Applying a Radiographic Definition for ARDS. Chest 1999; 116: 1347-1353.

214. Ryan SW, Nycyk J, Shaw BNJ. Prediction of chronic neonatal lung disease on day 4 of life. Eur J Pediatr 1996; 155: 668-671.

215. Ryan SW, Wild NJ, Arthur RJ, Shaw BNJ. Prediction of chronic neonatal lung disease in very low birthweight neonates using clinical and radiological variables. Arch Dis Child 1994; 71: F36-F39.

216. Sahebjami H. Nutrition and lung structure and function. Exp Lung Res 1993; 19: 105-124.

217. Schramm CM, Grunstein MM, Pulmonary function tests in infants. In: Kendig's disorders of the respiratory tract in children; Chernick V, editor. 1990.

218. Shennan AT, Dunn MS, Ohlsson A, Lennox K, Hoskins EM. Abnormal pulmonary outcomes in premature infants: prediction from oxygen requirement in the neonatal period. Pediatrics 1988; 82: 527-532.

219. Simbruner G, Coradello H, Lubec G, Pollak A, Salzer H. Respiratory compliance of newborns after birth and its prognostic value for the course and outcome of respiratory disease. Respiration 1982; 43: 414-423.

221. Smith TG, Proops DW, Pearman K, Hutton P. Hypoxia in sleeping children: overnight studies can be reduced to 4 hours without loss of clinical significance. Clin Otolaryngol 1992; 17: 243-245.

222. Smyth JA, Tabachnik E, Duncan WJ, Reilly BJ, Levison H. Pulmonary function and bronchial hyperreactivity in long-term survivors of bronchopulmonary dysplasia. Pediatrics 1981; 68: 336-340.

223. Smyth JA, Tabachnik E, Duncan WJ, Reilly BJ, Levison H. Pulmonary function and bronchial hyperreactivity in long-term survivors of bronchopulmonary dysplasia. Pediatrics 1981; 68: 336-340.

224. Sprikkelman AB, Grol MH, Lourens MS, Gerritsen J, Heymans HS, van Aalderen WMC. Use of tracheal auscultation for the assessment of bronchial responsiveness in asthmatic children. Thorax 1996; 51: 317-319.

225. Sprikkelman AB, Schouten JP, Lourens MS, Heymans HSA, van Aalderen WMC. Agreement between spirometry and tracheal auscultation in assessing bronchial responsiveness in asthmatic children. Respir Med 1999; 93: 102-107. 
226. Steyerberg EW, Eijkermans MJC, Habbema JDF. Stepwise selection in small data sets: a simulation study of bias in logistic regression analysis. J Clin Epidemiol 1999; 52(10): 935942.

227. Stick SM, Burton PR, Gurrin L, Sly PD, LeSouef PN. Effects of maternal smoking during pregnancy and a family history of asthma on respiratory function in newborn infants. Lancet 1996; 348: 1060-1064.

228. Stocks J. Recent advances in the assessment of lung function in infants. Pneumologie 1991; 45: 881-886.

229. Stocks J. Growth and development of the lungs - physiological aspects. Am J Respir Crit Care Med 1995; 151: S15-S17.

230. Stocks J, Godfrey S. Nasal resistance during infancy. Respir Physiol 1978; 34: 233-246.

231. Subramaniam P, Henderson-Smart DJ, Davis PG, Prophylactic nasal continuous positive airways pressure for preventing morbidity and mortality in very preterm infants (Cochrane Review). 2002; 2: Oxford, England: Update Software.

232. Tager IB, Hanrahan JP, Tosteson TD, Castile RG, Brown RW, Weiss ST, Speizer FE. Lung function, pre- and post-natal smoke exposure, and wheezing in the first year of life. Am Rev Respir Dis 1993; 147: 811-817.

233. Taussig LM, Helms PJ, Introduction. In: Infant respiratory function testing; Stocks J, Sly PD, Tepper RS, Morgan WJ, editors. 1996; 1-18.

234. Taussig LM, Landau LI, Godfrey S, Arad I. Determinants of forced expiratory flows in newborn infants. J Appl Phyiol 1982; 53: 1220-1227.

235. Tepper RS, Merth IT, Newth CJL, Gerhardt T, Measurement of functional residual capacity in infants by helium dilution and nitrogen washout techniques. In: Infant respiratory function testing; Stocks J, Sly P, Tepper RS, Morgan WJ, editors. 1996; 165-189.

236. Tepper RS, Pagtakhan RD, Taussig LM. Non invasive determination of total respiratory system compliance in infants by the weighted spirometer method. Am Rev Respir Dis 1984; 130: 461-466.

237. Tepper RS, with the technical assistance of Kisling J, Brearton L. Maturation affects the maximal pulmonary response to methacholine in rabbits. Pediatr Pulmonol 1993; 16: 48-53.

238. the Have-Opbroek AAW, Otto-Verberne CJM, Dubbeldam JA, Dijkman JH. The proximal border of the human respiratory unit, as shown by scanning and transmission electron microscopy and light microscopical cytochemistry. Anat Rec 1991; 229: 339-354.

239. The Impact-RSV study group. Palivizumab, a humanized respiratory synsytial virus monoclonal antibody, reduces hospitalization form respiratory syncytial virus in high-risk infants. Pediatrics 1998; 102: 531-537.

240. Thomassen MJ, Antal JM, Connors MJ, Meeker DP, Wiedemann HP. Characterization of Exosurf (Surfactant)-mediated suppression of stimulated human alveolar macrophage cytokine responses. Am J Respir Cell Mol Biol 1994; 10: 399-404.

241. Thurlbeck WM. Postnatal human lung growth. Thorax 1982; 37: 564-571.

242. Toce SS, Farrell PM, Leavitt LA, Samuels DP, Edwards DK. Clinical and roentgenographic scoring systems for assessing bronchopulmonary dysplasia. Am J Dis Child 1984; 138: 581-585.

243. Todisco T, de Benedictis FM, Iannacci L, Baglioni S, Eslami A, Todisco E, Dottorini M. Mild prematurity and respiratory functions. Eur J Pediatr 1993; 152: 55-58.

244. Tooley WH. Epidemiology of bronchopulmonary dysplasia. J Pediatr 1979; 95: 851-858. 
245. Translated by Singer C. Galen on anatomical procedures. Oxford: Oxford University Press 1956.

246. Tschanz SA, Burri PH. Postnatal lung development and its impairment by glucocorticoids. Pediatr Pulmonol 1997; 16: 247-249.

247. Tschanz SA, Damke BM, Burri PH. Influence of post-natally administered glucocorticoids on rat lung growth. Biol Neonate 1995; 68: 229-245.

248. Turney SZ, Blumenfeld W. Heated Fleisch pneumotachometer: a calibration procedure. J Appl Physiol 1973; 34: 117-121.

249. Technical specifications of the Nellcor N-395 pulse oximeter 2000; 's-Hertogenbosch, The Netherlands.

250. Vaida P, Bargeton D, Guénard H. BTPS calibration of heated Fleisch pneumotachometer. Bull Eur Physiopathol Respir 1983; 19: 635-640.

251. van Bel F. Toediening van glucocorticosteroïden aan de te vroeg geborene: steeds meer aanwijzingen voor ongewenste bijwerkingen. Ned Tijdschr Geneeskd 2001; 145: 2521-2525.

252. van der Ent CK, Brackel HJ, van der Laag J, Bogaard JM. Tidal breathing analysis as a measure of airway obstruction in children three years of age and older. Am J Respir Crit Care Med 1996; 153: 1253-1258.

253. van der Heide-Jalving M, Kamphuis PJGH, van der Laan MJ, Bakker JM, Wiegant VM, Heijnen CJ, Veen S, van Bel F. Short- and long-term effects of neonatal glucocorticoid therapy: is hydrocortisone an alternative to dexamethasone? Acta Paediatr 2003; 92: $827-$ 835.

254. van Houwelingen JC, le Cessie S. Predictive value of statistical models. Stat Med 1990; 8: 1303-1325.

255. van Kaam AH, de Jaegere A, Haitsma JJ, van Aalderen WM, Kok JH, Lachmann B. Positive pressure ventilation with the open lung concept optimizes gas exchange and reduces ventilator-induced lung injury in newborn piglets. Pediatr Res 2003; 53: 245-253.

256. van Lierde S, Smith J, Devlieger H, Eggermont E. Pulmonary mechanics during respiratory distress syndrome in the prediction of outcome and differentiation of mild and severe bronchopulmonary dysplasia. Pediatr Pulmonol 1994; 17: 218-224.

257. Verloove-Vanhorick SP, Verwey RA. Project on preterm and small for gestational age infants in the Netherlands 1983. 1987.

258. von Neergard K. Neue Auffassungen über einen Grundbegriff der Atem-mechanik. Die Retraktionskraft der Lunge, abhängig von der Oberflächen-spannung in den Alveolen. $Z$ Gesamte Exp Med 1929; 66: 373-394.

259. Wagner PD. Ventilation-perfusion relationships. Annu Rev Physiol 1980; 42: 235-247.

260. Walther FJ, Wagenaar GTM. Ontwikkelingsbiologie van de long: pathogenese van bronchopulmonale dysplasie. Tijdschr Kindergeneeskd 2002; 70: 39-45.

261. Warner JA, Jones AC, Miles EA, Colwell BM, Warner JO. Maternofetal interaction and allergy. Allergy 1996; 51: 447-451.

262. Watterberg KL, Demers LM, Scott SM, Murphy S. Chorioamnionitis and early lung inflammation in infants in whom bronchopulmonary dysplasia develops. Pediatrics 1996; 97: 210-215.

263. Wawersik J. Pneumotachographie, Anwendungsmöglichkeiten und methodische Grundlagen im Rahmen anaesthesiologischer Probleme bei Säuglingen und Kleinkindern. Anaesthesist 1965; Band 14: 259-263. 
264. Clinical decision analysis 1980; WB Saunders Company, Philadelphia.

265. Wetenschappelijke begeleidingscommissie van de Landelijke Neonatologie Registratie. Toename van het aantal vroeggeboorten in Nederland: vergelijking van 1983 en 1993. Ned Tijdschr Geneeskd 1998; 142: 127-131.

266. Wijnands JBG, Esajas M, Egberts J. Usefulness of the lecithin/sphingomyelin ratio and uselessness of phosphatidylglycerol determinations in tracheal aspirates of preterm infants. Clin Chem 1989; 35: 706.

267. Wilkie RA, Bryan MH. Effect of bronchodilators on airway resistance in ventilatordependent neonates with chronic lung disease. J Pediatr 1987; 111: 278-282.

268. Woodgate PG, Davies MW, Permissive hypercapnia for the prevention of morbidity and mortality in mechanically ventilated newborn infants (Cochrane Review). 2001; 2: Oxford, England: Update Software.

269. Yeh MP, Adams TD, Gardner RM, Yanowitz FG. Effect of O2, N2 and CO2 composition on nonlinearity of Fleisch pneumotachograph characteristics. J Appl Physiol 1983; 56: 1423-1425.

270. Yoder BA, Anwar MU, Clark RH. Early prediction of neonatal chronic lung disease: a comparison of three scoring methods. Pediatr Pulm 1999; 27: 388-394.

271. Yong SC, Smith CM, Wach R, Kurian M, Primhak RA. Methacholine challenge in preschool children: methacholine-induced wheeze versus transcutaneous oximetry. Eur Respir J 1999; 14: 1175-1178.

272. Yoon HB, Romero R, Kim KS, Park JS, Ki SH, Kim BI, Jun JK. A systemic fetal inflammatory response and the development of bronchopulmonary dysplasia. Am J Obstet Gynecol 1999; 181: 773-779.

273. Yost CC, Soll RF, Early versus delayed selective surfactant treatment for neonatal respiratory distress syndrome (Cochrane Review). 2002; 22: Oxford, England: Update Software.

274. Young S, Arnott J, O'Keeffe PT, Le Souef PN, Landau LI. The association between early life lung function and wheezing during the first 2 yrs of life. Eur Respir J 2000; 15: 151157.

275. Yuksel B, Greenough A, Gamsu HR. Respiratory function at follow-up after neonatal surfactant replacement therapy. Respir Med 1993; 87: 217-221.

276. Yuksel B, Greenough A, Karani J. Comparison of a subjective and a quantitative assessment of the neonatal chest radiograph. BrJ Radiol 1992; 65: 1083-1085.

277. Yuksel B, Greenough A, Karani J. Prediction of chronic lung disease from the chest radiograph appearance at seven days of age. Acta Paediatr 1993; 82: 944-947.

278. Zimmerman JJ, Farrell PM. Advances and issues in bronchopulmonary dysplasia. Curr Probl Pediatr 1994; 24: 159-170.

279. Zock JP. Linearity and frequency response of Fleisch type pneumotachometers. Pflugers Arch 1981; 391: 345-352.

280. Stick SM, Turnbull S, Chua HL, Landau LI, Le Souef PN. Bronchial responsiveness to histamine in infants and older children. Am Rev Respir Dis 1990; 142: 1143-1146.

281. Wilson NM, Bridge P, Phagoo SB, Silverman M. The measurement of methacholine responsiveness in 5 year old children: three methods compared. Eur Respir J 1995; 8: 364 370 . 


\section{References}

282. Springer C, Godfrey S, Picard E, Uwyyed K, Rotschild M, Hananya S, Noviski N, Avital A. Efficacy and safety of methacholine bronchial challenge performed by auscultation in young asthmatic children. Am J Respir Crit Care Med 2000; 162: 857-60. 


\section{Curriculum vitae}

Yvonne Snepvangers was born on 12 January 1972 in Steenbergen, The Netherlands. She graduated from Secondary School (Juvenaat Het Heilig Hart in Bergen op Zoom) in 1990. In the same year she started her medical studies at the University of Maastricht and obtained her Medical Degree in 1998. As part of her medical training, she studied incidence and survival rates in children with various kinds of malignancies throughout the period 1971-1995 in The Netherlands at the Eindhoven Cancer Registry (Integraal Kankercentrum Zuid). Later in 1998, she completed the postacademic course in order to work as a medical doctor in infant welfare centres. In January 1999, she started as a resident at the paediatric intensive care unit at the Radboud Medical Centre in Nijmegen, The Netherlands. In June of the same year, she began as a PhD-student at the Wilhelmina Children's Hospital, University Medical Centre Utrecht in Utrecht, The Netherlands, to study early postnatal respiratory mechanics in ventilated preterm infants and their relationship with respiratory morbidity and lung function during the first two years of life headed by Prof. dr. F. van Bel of the department of Neonatology and Prof. dr. J.L.L. Kimpen of the department of Paediatrics at the University Medical Centre Utrecht. In July 2003, she started her specialist training in paediatrics in the Sophia Hospital of the Isala Clinics in Zwolle, The Netherlands (head: dr. W. Baerts). From January 2005 she will continue the specialist training in the Wilhelmina Children's Hospital of the University Medical Centre Utrecht (head: Prof. dr. J.L.L. Kimpen). 



\section{Dankwoord}

"Graag wil ik iedereen bedanken die een essentiële bijdrage heeft geleverd aan de totstandkoming van dit proefschrift. Zonder jullie.... BEDANKT!"

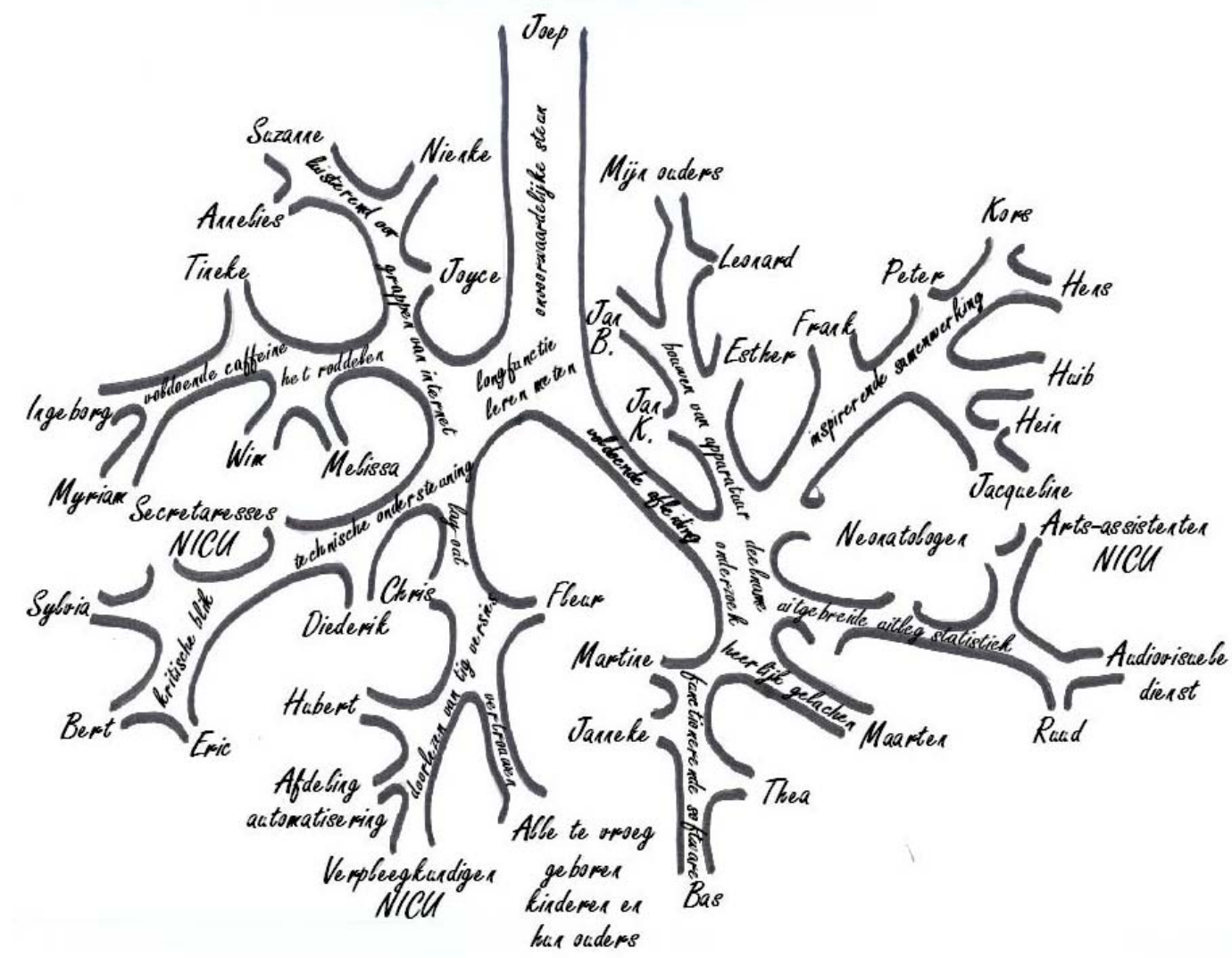

